\title{
ASSESSING THE COMMERCIALIZATION POTENTIAL OF ALGAL JETFUEL USING A LIFECYCLE ASSESSMENT APPROACH
}

\author{
By \\ Hossain Seraj, BSc., York University, October 2009
}

A thesis presented to Ryerson University

In partial fulfillment of the requirements for the degree of Master of Applied Science

In the program of Environmental Applied Science and Management

Toronto, Ontario, Canada, 2014

(CHossain Seraj 2014 


\section{AUTHORS DECLRATAION FOR ELECTRONIC SUBMISSION OF A THESIS}

I hereby declare that I am the sole author of the thesis. This is a copy of the thesis. Including any required final revisions, as accepted by my examiners.

I authorize Ryerson University to lend this thesis to other institutions or individuals for the purpose of scholarly research.

I further authorize Ryerson University to reproduce this thesis by photocopying or by other means,

in total or in part, at the request of other institutions or individuals for the purpose of scholarly research.

I understand that my thesis may be made electronically available to the public. 
Assessing the Commercialization Potential of Algal Jetfuel Using a Lifecycle Assessment Approach. MASc 2014. Hossain Seraj. Environmental Applied Science and Management. Ryerson University.

\section{ABSTRACT}

Farming algae for chemicals, pigments, neutraceutical and even fuel is not a novel idea. What is new however is recent volatility in energy prices coupled with heightened global sensitivity to food prices - partly instigated by the massive proliferation of food-based biofuels - that has brought algal biofuels to the forefront of energy research and commercial activity. Algal biofuels offer great promise in providing a sustainably-sourced, carbon-neutral option that can meet a significant portion, if not all, of the global transportation fuel needs in the coming decades. For a sector such as aviation, which has no other short-term practical alternative to fossil fuel liquid fuels, algal jetfuel offers a massive opportunity that if captured, can provide fuel cost and supply stability as well as a critical avenue to actively manage its growing GHG footprint. However, being a nascent technology, the fuel pathway innovation will rely on heavy and continuous investment to accelerate its development. This study assesses whether a carbon price framework can enhance the commercialization potential of algal jetfuel by way of mobilizing investment into the technology, and if not, what requisite improvements in technology and policy accommodations need to be made in order to allow algal jetfuel to be competitively produced. 
I would like to acknowledge Dr. Philip Walsh for his continuing support, guidance and mentorship in my pursuits through my graduate studies. I would also like to acknowledge the Environmental Applied Science and Management Program, and Yeates School of Graduate Studies for the financial support that enabled my development during this period. 
To my family and Anda for the unconditional love and support. 


\section{TABLE OF CONTENTS}

LIST OF TABLES

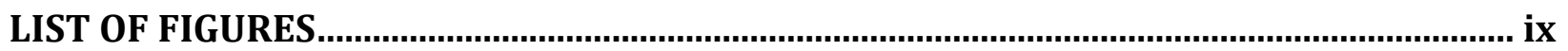

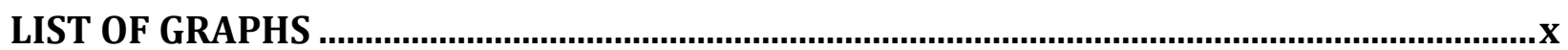

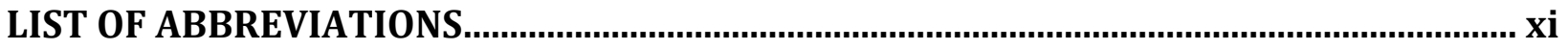

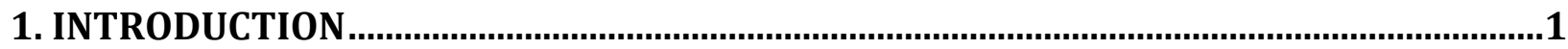

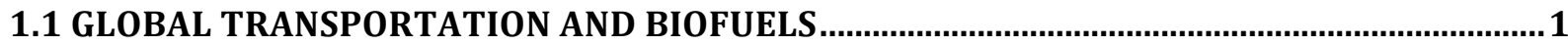

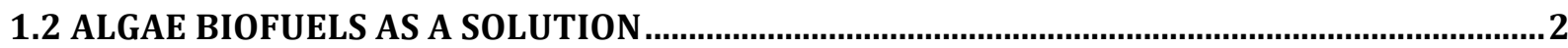

1.3 ADVANTAGES OF ALGAL FEEDTSOCK..................................................................................

2. ALGAL BIOFUELS AND AVIATION

3. THE RESEARCH QUESTION

4. THEORETICAL FRAMEWORK

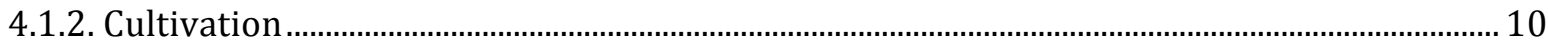

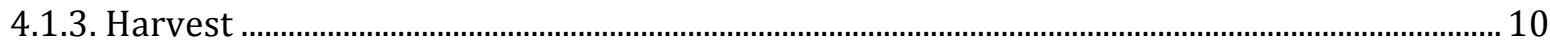

4.1.4. Oil Extraction and Recovery ................................................................................................. 11

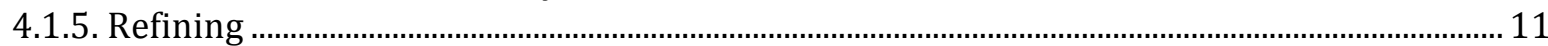



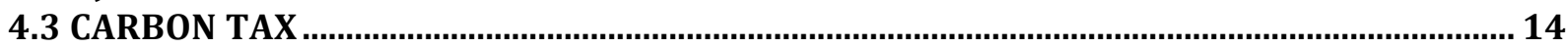

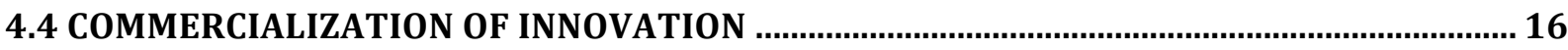

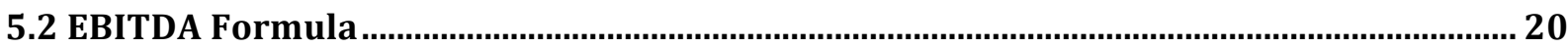

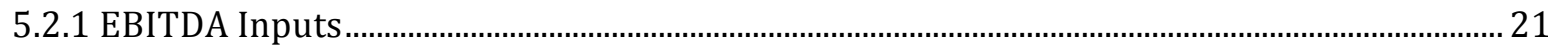

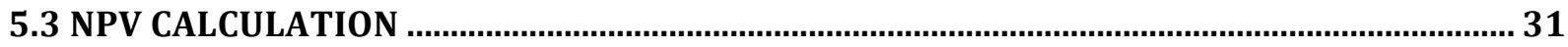

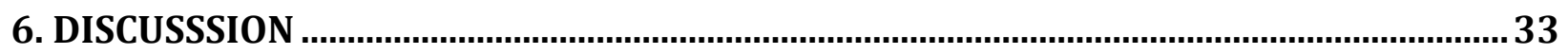

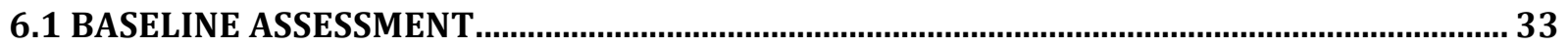

6.2.3 Impact of Changing JFP and Carbon Tax on NPV ............................................................. 38

6.2.4 Impact of Changing Growth Scenario on NPV .................................................................. 41

6.2.5 Impact of Changing Growth Scenario and JFP on NPV .................................................. 43

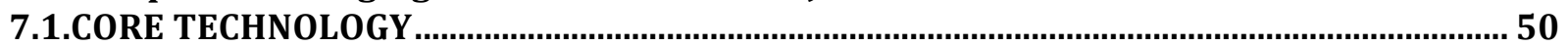

7.1.2 Raise OPEX Efficiency......................................................................................................... 52

7.2 POLICY RECOMMENDATIONS.......................................................................................59

7.2.1 Compliment Carbon Tax with Other Policy Measures ...................................................................59

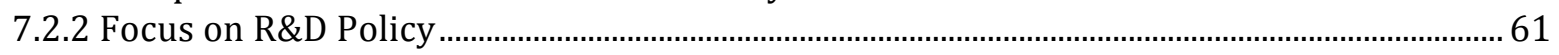

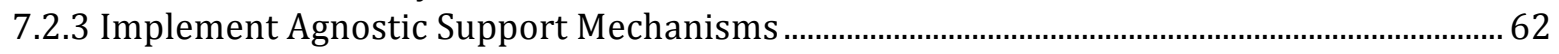



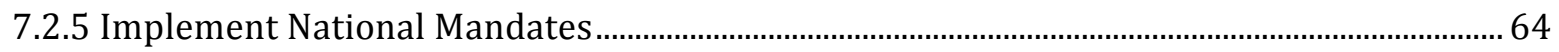

7.2.6 Implement Emission Standards ..................................................................................6

7.2.7 Model Technology Policy Around Investor Interests .....................................................67

7.2.8 Assimilate Considerations for Investor Psychology in Investment Policy ..........................68

7.2.9 Create Cross-Disciplinary and Cross-Regional Partnerships ..............................................71

9. CONCLUSION

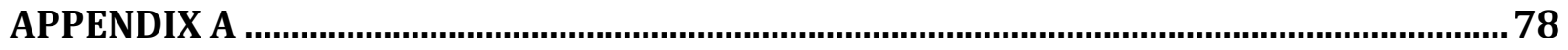






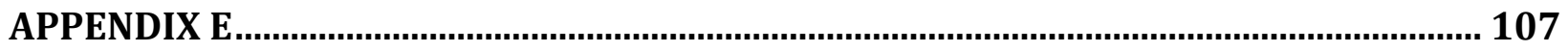

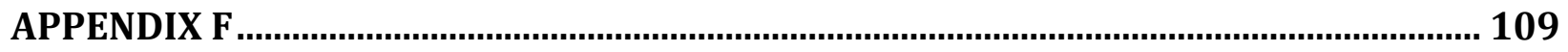

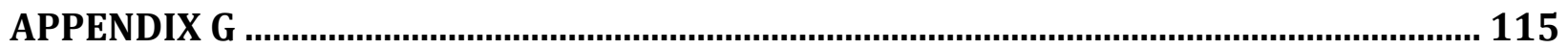

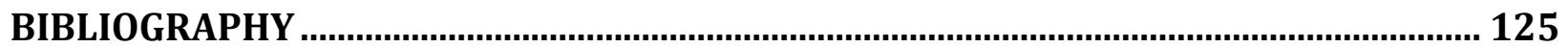




\section{LIST OF TABLES}

TABLE 1 - US DOE EERE EVALUATION OF ALGAE TECHNOLOGY

TABLE 2A - BASELINE CONDITIONS

TABLE 2B - BASELINE NPV ASSESSMENT

TABLE 3A - JFP VS. NPV CONDITIONS

TABLE 3B - JFP VS. NPV ASSESSMENT

TABLE 4A - CARBON TAX VS. NPV CONDITIONS

TABLE 4B - CARBON TAX VS. NPV ASSESSMENT

TABLE 5A - JFP \& CARBON TAX CONDITIONS

TABLE 5B - JFP \& CARBON TAX VS. NPV ASSESSMENT

TABLE 6A - GROWTH SCENARIO CONDITIONS

TABLE 6B - GROWTH SCENARIO VS. NPV ASSESSMENT

TABLE 7A - GROWTH SCENARIO \& JFP CONDITIONS

TABLE 7B - GROWTH SCENARIO \& JFP VS. NPV ASSESSMENT

TABLE 8A - GROWTH SCENARIO \& CARBON TAX VS. NPV

TABLE 8B - GROWTH SCENARIO \& CARBON TAX CONDITIONS 


\section{LIST OF FIGURES}

FIGURE 1 - MAPPING OUT AVIATION'S GHG COMMITMENTS

FIGURE 2 - FORECAST FOR FUEL COSTS CAPTURING POTENTIAL COST OF CARBON

FIGURE 3A - CORN ETHANOL VALUE CHAIN

FIGURE 3B - AJF VALUE CHAIN

FIGURE 4 - TYPICAL DISTILLATION RANGES (DEGREES C) AND CARBON-NUMBER RANGES FOR FUELS

FIGURE 5- WTP, PTW, WTW DEFINITIONS FOR ALGAE BIOFUEL

FIGURE 6 - APD DEFINED BY GREET

FIGURE 7- DEFAULT PRE-CONFIGURED APD DEFINED BY GREET

FIGURE 8 - GREET WTWA EVALUATION FOR ALTERNATIVE JET FUEL PRODUCTION PATHWAYS

FIGURE 9 - COST SENSITIVITY TORNADO PLOT FOR KEY CAPEX AND OPEX FACTORS 


\section{LIST OF GRAPHS}

GRAPH 1 - JFP VS. NPV

GRAPH 2 - CARBON TAX VS. NPV

GRAPH 3 - JFP \& CARBON TAX

GRAPH 4 - GROWTH SCENARIO VS. NPV

GRAPH 5 - GROWTH SCENARIO \& JFP VS. NPV

GRAPH 6 - GROWTH SCENARIO \& CARBON TAX VS. NPV 


\section{LIST OF ABBREVIATIONS}

JFP - Jetfuel Price

AJF - Algal Jetfuel

PJF - Petroleum Jetfuel

NREL - National Renewable Energy Laboratory

US DoE - United States Department of Defense

$\mathrm{CO}_{2}$ - Carbon Dioxide

$\mathrm{SO}_{\mathrm{x}}-$ Sulphur Oxide

$\mathrm{CH}_{3}$ - Methane

ATAG - Air Transport Action Group

IATA - International Air Transport Association

IEA - International Energy Association

EISA - Energy Independence and Security Act

EU RED - European Union Renewable Energy Directive

DAF - Dry Air Flocculation

LEA - Lipid Extracted Algae

HEFA - Hydroprocessed Ester of Fatty Acids

Bio-SPK - Bio Synthetic Paraffinic Karosene

ASTM - American Society for Testing and Materials

ANL - Argonne National Laboratory

SAFUG - Sustainable Aviation Fuel User Group

CSIRO - Commonwealth Scientific and Industrial Research Organization

GDP - Gross Domestic Product

IPCC - Intergovernmental Panel of Climate Change

BC - British Columbia

R\&D - Research \& Development

RET - Renewable Energy Technology

CAPEX - Capital Expenditure

OPEX - Operational Expenditure

WACC - Weighted Average Cost of Capital

NPV - Net Present Value

DCF - Discounted Cash Flow

PV - Present Value

$\mathrm{CF}$ - Cash Flow

FCF - free Cash Flow

EBITDA - Earnings Before Interest, Tax, Depreciation and Amortization

UCC - Upfront Capital Cost

TV - Terminal Value

COP - Cost Of Production

DoE EERE - Department of Defense Energy Efficiency and Renewable Energy

COR - Cost Of Refining

TEA - Technoeconomic Assessment

LCA - Lifecycle Assessment

RA - Resource Assessment 


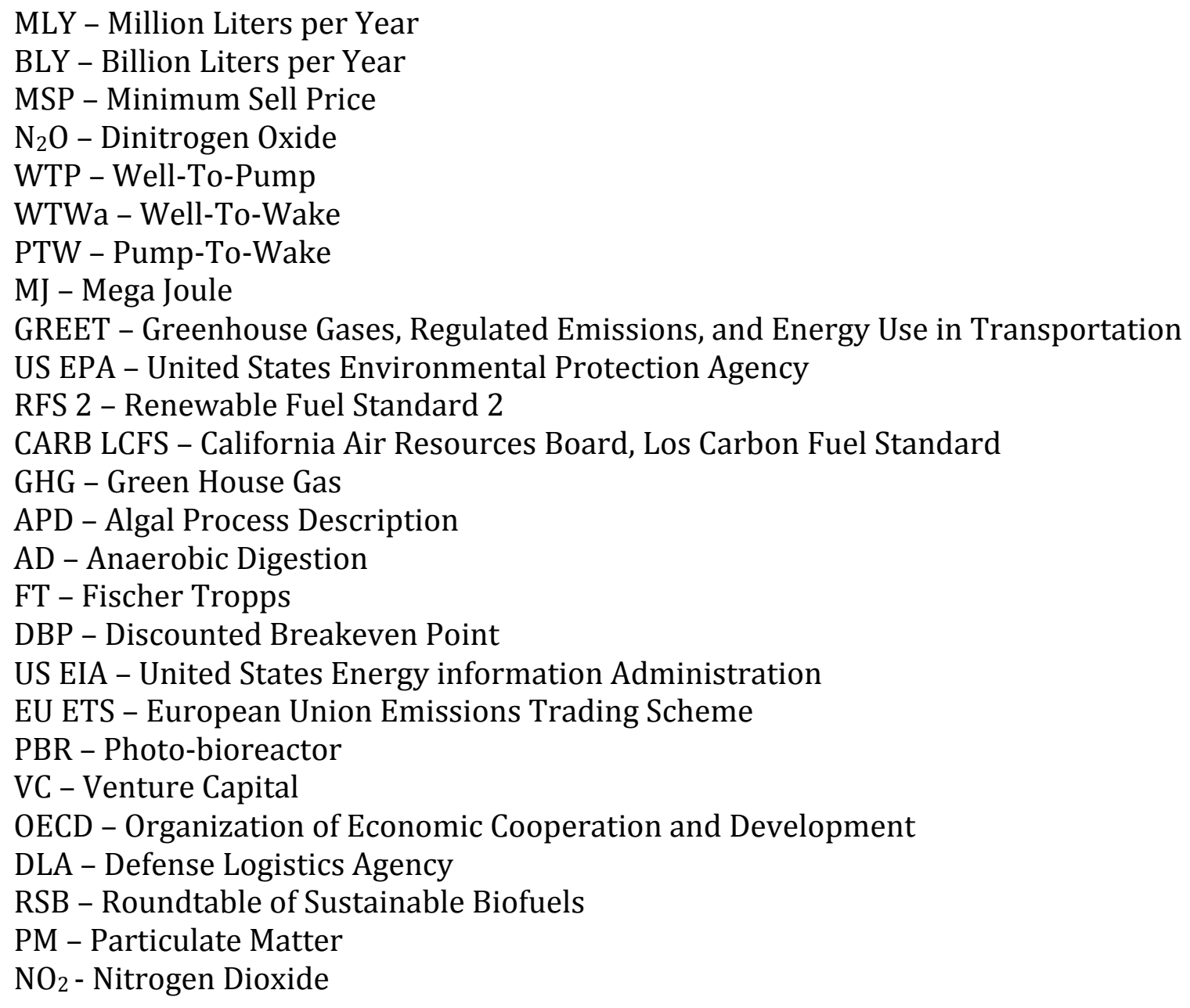




\section{INTRODUCTION}

\subsection{GLOBAL TRANSPORTATION AND BIOFUELS}

The world population is set to rise to 10 billion people by 2050 (UN, 2010). World energy consumption in the transportation sector is set to rise by an average of $1.1 \%$ per year. Petroleum and other liquid fuels are the most important component of transportation sector energy and will account for the largest share - 63\% - of the total growth in world consumption of petroleum and other liquid fuels from 2010 to 2040 (EIA, 2013). While volatile crude oil prices in the last few decades have brought matters of energy security into focus, more recently a growing acceptance of climate change related impacts triggered by ceaseless $\mathrm{CO}_{2}$ emissions has also impinged on the urgency to address sustainable alternatives to conventional transportation fuels.

Unlike the electricity market where multiple low carbon intensive technologies such as nuclear and hydroelectric power exist, carbon neutral production systems in the transportation sector are far less developed (Schenk et al, 2008). While some advancement in battery technology has allowed for limited utilization of electricity, it is widely accepted that the vast majority of transportation vehicles will continue to depend on liquid fuels well into the near future (Fairley, 2011). In this regard, biofuels provide a vital substitute.

Biofuel production has undergone massive proliferation in the last decade surging up from 16 billion liters in 2000 to 100 billion liters produced in 2010. This feat has been largely achieved by production capacities in the US, the EU and Brazil where significant infrastructure to cultivate, process and refine feedstock (raw materials) exist (IEA, 2011). According to the 2011 International Energy Association (IEA) Biofuels Technology Roadmap, “conventional biofuel technologies include well-established processes that are already producing biofuels on a commercial scale. These biofuels commonly referred to as first-generation, include sugar- and starch-based ethanol, oil-crop based biodiesel and straight vegetable oil, as well as biogas derived through anaerobic digestion. Typical feedstocks used in these processes include sugarcane and sugar beet, starch-bearing grains like corn and wheat, oil crops like rape (canola), soybean and oil palm, and in some cases animal fats and used cooking oils" (IEA, 2011, pg. 8). However, the massive proliferation of $1^{\text {st }}$ generation biofuels has not come without significant social costs and controversy. The rush to develop biofuels has diverted a substantial amount of agricultural resources from food production towards biorefineries. In the US, one third of the 92 million acres of corn cultivated per year is allocated towards ethanol production. Similar large-scale diversions in major food producing countries have instigated some of the highest spikes in food prices in the last twenty years (Graham-Rowe, 2011). Numerous high profile 
scientific studies also indicate that prolific cultivation of food-based feedstock for energy production may have yet unrealized indirect climate change impacts as the profitability of energy crops prompt many farmers to shift away from commodity food crops to fuel crops, eliciting a ripple effect whereby compensatory food production is extended to forested areas and non-conventional agricultural lands. In Malaysia and Indonesia, heightened biofuel consumption and favorable government policies have indirectly triggered extensive deforestation to accommodate palm oil production (Graham-Rowe, 2011). Full lifecycle analyses of conventional $1^{\text {st }}$ generation biofuels also report minimal GHG savings and in some cases, even greater GHG emissions when compared to fossil fuel equivalents (Fairley, 2011).

\subsection{ALGAE BIOFUELS AS A SOLUTION}

Whilst recent notable spikes in food prices and devastating indirect effect of $1^{\text {st }}$ generation biofuels ushered public scrutiny and slowed the development of newer biofuel technologies, disasters in Japan and the Gulf of Mexico as well as price volatility and geopolitical instability in oil producing regions have reemphasized the role of biofuels in the global energy mix. The IEA reports that the expansion of biofuel consumption from $2 \%$ to $27 \%$ of global transportation fuels by 2050 could displace enough petroleum to avoid 2.1 gigatons of $\mathrm{CO}_{2}$ about as much as the net $\mathrm{CO}_{2}$ absorbed by the ocean every year (Fairley, 2011). Favorable government policies, environmental legislations and emission standards in the biggest biofuel markets serve as good indicators of biofuel development in the years to come. The European Commission with its Renewable Energy Directive (RED) mandates $10 \%$ of its transportation fuels to be derived from biofuels but specifically bars import of fuels produced through unsustainable practices. Similarly, the US Energy Independence and Security Act (EISA) and its Renewable Fuel Standard (RFS) derivative mandates consumption of at least 36 billion gallons of renewable fuel by 2022 while capping the production of $1^{\text {st }}$ generation fuels (mostly corn bioethanol) at 15 billion gallons ( $\mathrm{Ng}, 2010)$. Additionally, to incentivize innovation in newer biofuel technologies, the EISA calls for at least 21 billion gallons of the 36 billion gallons to come from advanced biofuels (Preiss, 2010). Algae biofuels are an advanced technology candidate.

Algae are microscopic photosynthetic organisms that fix sunlight and $\mathrm{CO}_{2}$ into oils, carbohydrates, proteins and biomass (Schenk et al, 2008). Unlike higher plants, algae have extremely efficient photosynthetic light harvesting components and a high solar conversion efficiency (amount of sunlight converted to biomass) of $1-8 \%$ compared to $1 \%$ in terrestrial crops. Algae convert captured biological inputs into oils that can be transformed to biodiesel or jetfuel using standard transesterification or hydrocracking techniques respectively (Packer, 2009). Commercial algae cultivation for fuel is considered incredibly promising and pose a number of advantages over $1^{\text {st }}$ generation feedstock. 


\subsection{ADVANTAGES OF ALGAL FEEDTSOCK}

\section{Significantly Higher Productivity}

Algae can capture $95 \%$ of exposed light and covert up to $1-8 \%$ of the solar energy to biomass, compared to $1 \%$ in most terrestrial crops. Algae productivity, depending on the oil content (20-80\%), can range between 15-300 times the productivity of $1^{\text {st }}$ generation feedstocks like corn and soybean on a per hectare per year basis (Packer, 2009); it is estimated that between 2-6\% of the total US cropping area would be sufficient to produce enough algal oil to entirely satisfy its transportation fuel needs (Preiss, 2010).

\section{Requires Negligible Land Use Requirements}

Algae cultivation is not dependent on traditional agricultural land input and can occur in marginal land across diverse climatic conditions including desserts. Future commercial algal farming for fuel will not compete with food crops and in fact, the significantly large productivity will allow better utilization of remaining arable land (Graham-Rowe, 2011).

\section{Provides High $\mathrm{CO}_{2}$ Sequestration}

$\mathrm{CO}_{2}$ is a growth-limiting factor for algae and as a result the organism has developed efficient mechanisms to selectively capture $\mathrm{CO}_{2}$ into its biomass. Large algae farms can act as carbon sequestration units where flue gases carrying emissions from carbon intensive industries can be fed directly into algae growing vessels for sequestration. Algae have a high tolerance for $\mathrm{NO}_{\mathrm{x}}$ and mercury content typical of flue gases. Although $\mathrm{SO}_{\mathrm{x}}$ concentrations above 400ppm can negatively affect the culture pH (Packer, 2009), the sulfur content can be managed through pollution control mechanism in industrial installments. Pond Biofuels, based in Toronto, has built the world's first pilot facility that uses large algae reactors to sequester emissions from a neighboring cement factory. Pond Biofuel's reactors can sequester up to two tons of $\mathrm{CO}_{2}$ for every ton of algal biomass grown (Pond Biofuel, 2011), a figure corroborated by reports noting one hectare of algae ponds have the potential capacity to sequester one ton of $\mathrm{CO}_{2}$ (Schenk et al, 2008). To add further perspective, based on conservative estimates, two billion tons of $\mathrm{CO}_{2}$ can be sequestered by growing algae in the space equivalent to the entire land allocated to soybean crop (63.3 million acres) in the US (Knoshaug, 2011).

\section{Cultivation is Flexible with Water Quality}

Unlike plant based feedstocks, algae cultivation does not rely solely on freshwater and can be undertaken using alternative sources such as seawater and wastewater from water treatment plants. In fact, marine algae strains are able to absorb vital nutrients such as nitrogen and phosphorus from both seawater and wastewater (Schenk et al, 2008). 


\section{ALGAL BIOFUELS AND AVIATION}

The aviation industry, much like the ground transportation sector, is integral to the global economy. In 2010 the aviation sector fostered 56.6 million jobs globally and generated $\$ 2.2$ trillion in economic activity which constituted 3.5\% of the global GDP. Aviation-enabled trade of time sensitive goods provides a lifeline for a number of developing countries exporting perishable items such as food and flowers as major tradable commodities. It is estimated that about 1.5 million livelihoods in Africa rely on such direct aviation enabled trade with the UK alone (ATAG, 2012).

However, the aviation sector also currently accounts for 2\% of global GHG emissions (ATAG, 2009) and 5\% of total anthropological climate forcing (Dallara, 2011). The industry anticipates continuous sector growth at an average rate of $5 \%$ over the coming decades to be driven largely by rising demand for air transport in Asia Pacific. But despite great strides in fuel efficiency and engineering innovations expected in the same period, aviation's GHG footprint is set to grow to 3\% (ATAG, 2009) of all global emissions by 2050 (Dallara, 2011). It is likely that the jump in aviation's relative contribution to global $\mathrm{CO}_{2}$ emissions will accompany a period characterized by heightened sensitivity to climate change that will inevitably reinforce public and political pressure on the sector to drastically mitigate its GHG footprint. In terms of strategy, this presents aviation with the daunting task of creating a sustainable air transport system that not only poses minimal environmental impacts, but one that also satisfies the transportation needs of a globally connected society while providing adequate returns on investment to attract (and retain) investors, employees and a supporting value chain (Bonnefoy, 2011).

Aviation is vested in biofuels for three major reasons. Unlike ground transportation where prospects for wide scale electrification exist, the aviation sector does not have access to any feasible or cost effective alternative to conventional petroleum jetfuel, and is therefore likely to depend on liquid fuels for some time (ATAG, 2011). Biofuels provide a practical sustainable option. Secondly, fuel is the single largest source of expenditure for airlines comprising about $30 \%$ of all its operating costs, which combined with supply side constraints (only $5 \%$ of world oil production earmarked for jet fuel production) makes the industry very vulnerable to volatility in price, supply and quality of Petroleum Jetfuel (PJF) (IATA Fuel Report, 2012). Moreover, the sector expects that the forthcoming crude oil supply crunch and shift in climate change policy in major markets will greatly raise the price of jetfuel. Biofuels in this sense will provide means to regionalize fuel production and stabilize supply. Lastly, biofuels are a practical and crucial component of aviation's GHG mitigation strategy. As Figure 1 illustrates, biofuels are the only instrument amongst a handful of potential strategies that can impart the level of emission mitigation that is requisite to meeting target the International Air Transportation Association - a trade association representing 240 members constituting $84 \%$ of all global air traffic - has set for itself (ATAG, 2012). 


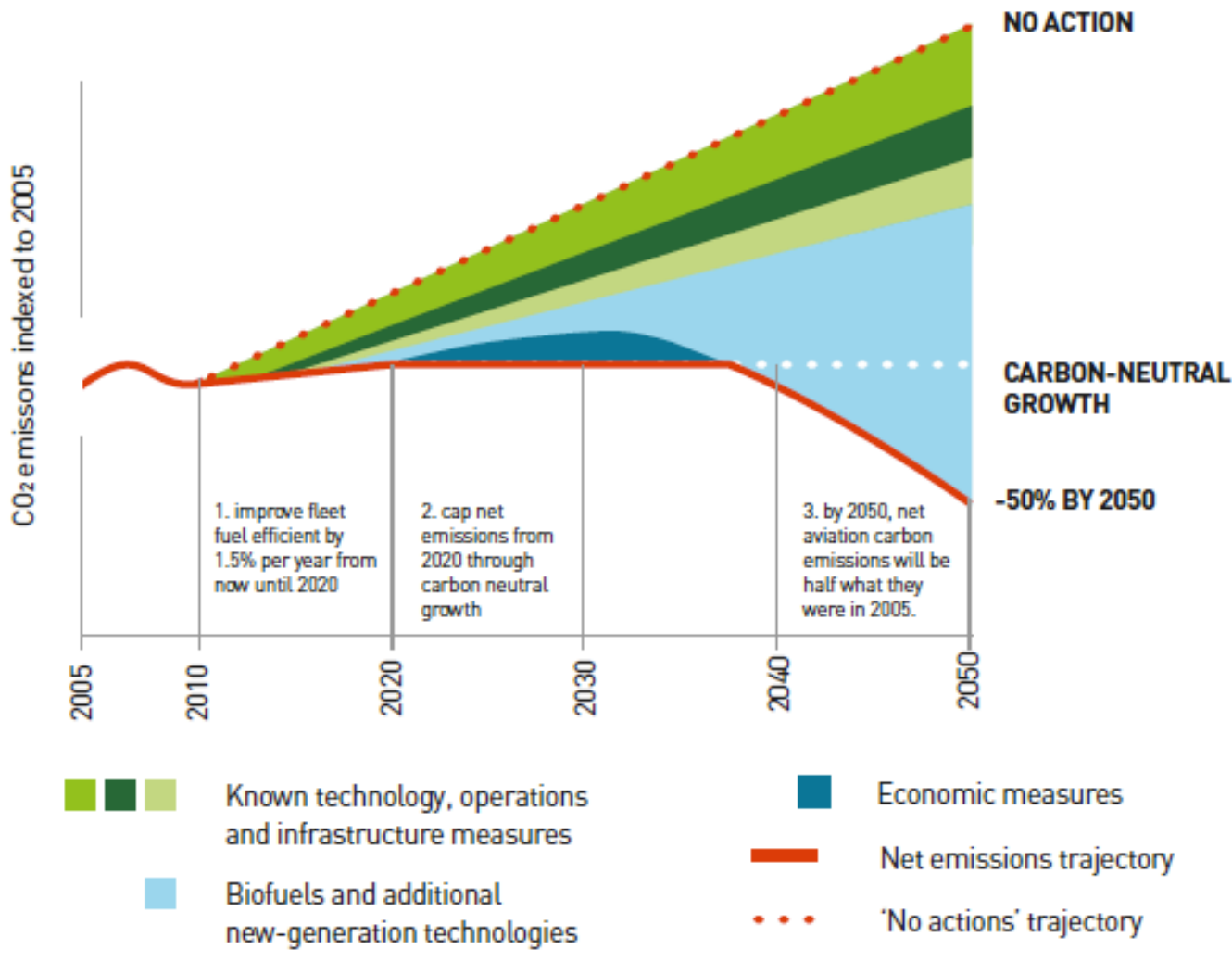

Figure 1 - Mapping Out Aviation's GHG Commitments (Source: ATAG 2012).

Currently these limiting factors have only a marginal effect on the aviation's operations but in anticipation of significant market pressures and political scrutiny, the industry is aggressively implementing forwardlooking economic measures to preemptively foster an aviation biofuel industry. Although sustainable bioderived jetfuels are not yet cost effective, the aviation sector expects with aggressive investment in R\&D and scale up of infrastructure, significant economies of scale will be reached within the near future (ATAG 2011).

Biofuels derived from algal feedstock are one subset of advanced biofuels that show promise in addressing aviation needs. According to Paul Steele, the Executive Director of Air Transport Action Group (the only global organization representing all part of the commercial air transport sector), "Algae has been identified as one of the most promising alternatives so we are eager to see how it performs" (ATAG Press Release, 2009). Algal Jetfuel (AJF) R\&D is currently being spearheaded by aviation funded activities. 


\section{THE RESEARCH QUESTION}

The biofuel types most in demand are high-quality diesel and jetfuel since few functional fuel analogues for them exist. Currently the cultivation of algal biomass and extraction of its oil - the feedstock for both end-use fuel types - is very expensive; excluding the costs for biofuel refining, production cost estimates for raw algae oil can vary between $\$ 0.75 / 1$ to more than $\$ 5 / 1$ creating an unlikely commercialization scenario for at least another 10-12 years. In the absence of commercial-scale algal fuel operations, the optimization, modeling and scale-up strategies for an algal jetfuel production pathway remains uncertain and requires continuous basic R\&D efforts and investment (IEA 2011) to alleviate the pressures of high cost. Additionally, AJF's commercialization potential depends largely on its market price relative to PJF which at present is not only very cheap to produce but also does not capture the inherent lifecycle environmental costs associated with its use, a benefit that is imparted by AJF but not captured or captured to a very limited extent in its market price under currently policy environments. This forms the basis of my research question: Can the commercialization scenario for AJF become more attractive under a policy framework where carbon emissions from jetfuel combustion are priced at relatively significant levels? To explore this question the emissions profile of AJF is incorporated into the financial valuation of a hypothetical AJF value chain to elucidate the impact a potential carbon price will have on the profitability of AJF production. Because new innovations rely heavily on attracting private capital through the promise of a return-on-investment to push product development, the commercialization of AJF will depend on the profitability it can offer to its producers and investors. 


\section{THEORETICAL FRAMEWORK}

The use of algae as an energy bearing crop for the production of biofuels is not a novel idea. It was first proposed by the Aquatic Species Program funded by the US Department of Energy (US DoE) in the 1980s (Knoshaug 2011) but the program's findings resurfaced and sparked a genesis of interest due to recent volatility in crude oil prices, environmental concerns and spiking food prices. Algae can be cultivated in freshwater, saline or wastewater and requires little or none of the agricultural inputs required by conventional biofuel crops (Carriquiry et al, 2011). Algae is actively being investigated as a model fuel producing organism for its voracious appetite for $\mathrm{CO}_{2}$ and ability to transform multiple waste streams to high quality cellular constituents that can then be processed into biodiesel, bioethanol or AJF. Prompted by the EISA and an advanced biofuel mandate, the US has become a hotbed for algal biofuel research; as of 2008 seven prominent US government laboratories, thirty US universities, and around sixty biofuel companies have been conducting research in this arena, complimented by intense regional efforts in Australia, Europe, the Middle East, and New Zealand (Carriquiry et al, 2011).

Notwithstanding the proliferation of applied algal fuel research, by and large a major roadblock for full-proof economic analysis of algae cultivation for fuel is the lack of available or verifiable information on performance due to proprietary technologies under development or simply the absence of any such commercial scale facility (Richardson et al, 2012). Most data quoted in the literature stem from lab and pilot scale investigations, many of which adopt process assumptions from current cultivation operations serving the food and nutraceuticals markets to simulate performance data for algal fuel cultivation (O'Connell \& Haritos, 2010). Naturally these assessments are limited in the extent to which they can emulate the true performance and variable risks inherent to AJF production. Risks in this context include (but are not limited to) i) production risk (growth medium health, quantity of biomass, oil content and co-product yields), ii) input prices $\left(\mathrm{CO}_{2}\right.$, labor, nutrients, water, electricity, construction, and maintenance), iii) output prices (algal oil and coproduct prices), iv) resource availability (water, $\mathrm{CO}_{2}$, and land), and v) climate and location (days of operation, net evaporation, and temperature) (Richardson et al, 2012). The commercialization and mass adoption of AJF can be seen as a multistage pathway through which the fuel technology innovation must traverse in order to enter the fuel market as a mature and profitable venture (Vertes et al, 2010). From an investor perspective, the multi-stage risks coupled with the absence of any commercial scale projects and incomplete accessibility to proprietary data (from private entities pursuing pilot tests) that could otherwise shed light on the potential performance of real life fuel operations results in an incomplete financial profile of AJF as a current business opportunity, which in turn discourages a pipeline of further investment that is vital to AJF's continuing maturity (Vertes et al, 2010).

As discussed, aviation's primary motivations for embracing AJF is to attain fuel cost stability, enhance fuel supply security, and to use AJF as a GHG management tool to mitigate rising sector emissions anticipated to 
grow from 2\% to 3\% of all global GHG emissions by 2050 (ATAG 2011). Aviation's commitment to emissions targets is largely pushed by its anticipation of inevitable shifts in emission policy that will likely instigate elevated carbon prices and heighten public pressure to operate 'better'. This study attempts to explore the central question, can AJF under its nascent technological state become financially feasible and profitable to produce under a hypothetical carbon price framework? And if not, what technological improvements and policy conditions are requisite to positing AJF in a cost competitive level with PJF? These questions are explored through the impacts of perturbing external factors on the profitability of a conceptual AJF value chain. In theory (Figure 2), a carbon price that penalizes $\mathrm{CO}_{2}$ equivalent emissions offers low carbon intensity options like AJF a boost in economic competitiveness; from aviation's perspective, a carbon price will incentivize aviation to shift its consumption from PJF to AJF in an attempt to save higher fuel costs incurred from emission penalties. From the AJF producer's perspective, a carbon price will create a demand for the product and hence aid in generating larger revenue. Because aviation is heavily committed to seeing the successful deployment of AJF (amongst other advanced biofuel technologies) both in terms of financial investment and policy engagement/development, for the purpose of this study, it can be safely assumed that a hypothetical AJF value chain is a direct extension of aviation's role in the arena i.e. aviation invests in the AJF value chain making the industrial sector an AJF producer.

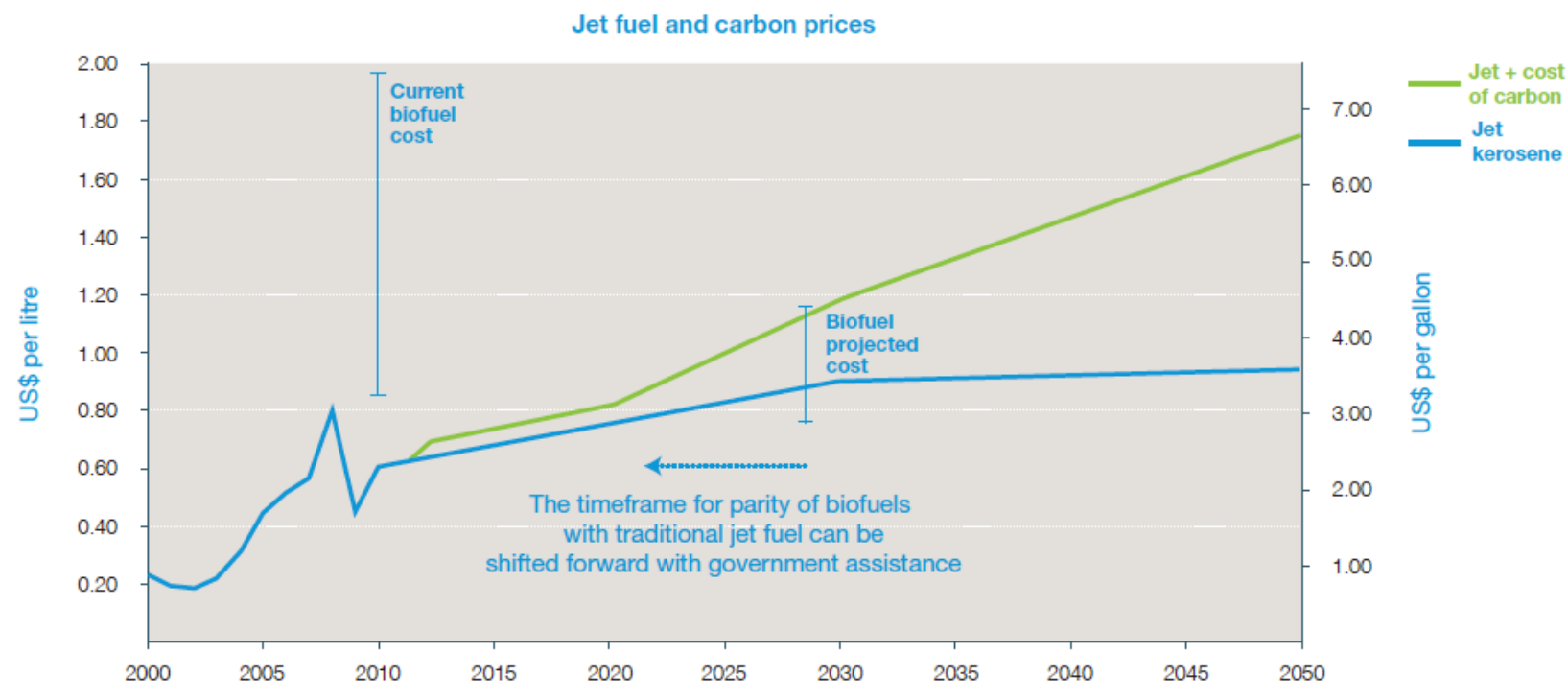

Figure 2 - Forecast for Fuel Costs Capturing Potential Cost of Carbon (Source: ATAG, 2009).

This study borrows data from major technoeconomic studies to build estimations for production costs but also moves beyond process and directly associated economic parameters (typical of technoeconomic studies) by including influence of external policy measures to determine the combination of conditions requisite to the 
feasible operation of a hypothetical commercial scale AJF value chain (Richardson et al, 2012). The research presented here rests on simplified assumptions, foregoing a comprehensive representation of the biofuel landscape in favor of an easy to understand depiction of the biofuel value chain to accommodate the scope of the study. This approach is justified by the fact that at this early stage in AJF innovation, more investment may be placed into understanding basic attributes of the algal biomass production systems than the entire value chain itself; as will become evident, there is less value in inventing a novel supply chain if the underlying harvesting or conversion mechanisms are yet to be identified and/or optimized. The economics of precommercial investment and rollout phases, to development and implementation will be critical as AJF research matures and upstream stages are optimized (O'Connell \& Haritos, 2010). Subsequently, the recommendations made here can guide activities undertaken by research organizations, industrial sectors or governments in their effort to chart a roadmap for AJF commercialization.

\subsection{ALGAL OIL PRODUCTION SCHEME}

The following section briefly articulates a typical algal oil production process to transform raw cultivated algae to the final AJF. As it stands now, a typical algal oil production process amalgamates multiple stages and technologies that are not exclusive to its production; the preliminary algal oil production stage borrows process techniques from commercial scale wastewater or fish feed manufacturing operations where algae cultivation serves in non-fuel producing markets. Consequently, as proven by pilot and demonstration projects, this conventional multi-stage regime has multiple points of inefficiency but will improve drastically as newer process technologies are developed to optimize algal oil productivity specific to fuel derivatives. Having said that, it is worthwhile to appreciate the algal oil production process as it is likely to exist in the short term.

\subsubsection{Strain Selection}

The first step demands identifying and culturing the optimal algal strain with attributes most suited to the growth medium and system conditions used in the production scheme. Ideally the right strain has a combination of high oil content, high growth rate and allows easy harvest and extraction of oil. Algae can grow in freshwater, seawater or wastewater mediums and so depending on the growth medium the specific algae strain with adequate tolerance for the growth conditions is selected i.e. if seawater is to be used as the growth medium, salt tolerant marine species are most suitable (Greenwell 2011). There is a trade off between growth rate and oil content; algae cells direct metabolic activity to growth and division in times when sunlight, $\mathrm{CO}_{2}$ and nutrients (mainly Nitrogen and Phosphorus) are plentiful and only switch gears to energy storage in form of oil and carbohydrate production when growth sustaining conditions don't exist. The cultivation stage often emulates starvation conditions and so sacrifices growth to maximize lipid content 
prior to harvest (Greenwell 2011). The type of oil produced is also an important consideration for the type of fuel derivative as the chemical characteristic of the oil dictates the stability and performance of the end-use product. For example in terms of meeting international fuel standards (Greenwell 2011) such as those set by American Society for Testing and Materials (Schenk et al 2008), the oxidative stability, flash point and freeze point are critical criteria for the type of extracted algal oil that be can used for aviation fuels.

\subsubsection{Cultivation}

Strains selection and master batch culture is followed by inoculation of the growth medium and cultivation in open raceway-style ponds that are only $0.2-0.3 \mathrm{~m}$ deep and vary in areal dimensions between $0.5-1$ hectare (Greenwell 2011). Ponds are usually built in areas with high solar incidence, paddle mixed, and depending on intensity of operation, have delivery systems attached that pump $\mathrm{CO}_{2}$ directly into the ponds (Greenwell 2011) to supplement nutrient requirement. Open ponds are simple and cheap to build thus have become the dominant method of algae cultivation for neutraceutical, animal feed and fuel production (Schenk et al 2008).

Conversely, open ponds by virtue of being open systems suffer from multiple inefficiencies that result in suboptimal biomass productivity and a large water footprint (Greenwell 2011). Open ponds lose water continuously through evaporation and thus require continuous replenishment to avoid dilapidating effect of rising growth medium salinity (Packer 2009). Open ponds are susceptible to seasonal variations in weather and temperature and therefore make consistent levels of cultivation difficult. More importantly, invasion and displacement by foreign bacteria, fungi, plants and other algal species can also impact culture integrity. Resilient algal strains can offer some relief but must be used at a price as more competitive algal species allocate metabolic activity to resiliency rather than growth (Knoshaug 2011).

\subsubsection{Harvest}

Algae cultures typically are very dilute cellular concentrates which necessitate dewatering prior to downstream processing. The main dewatering techniques employed are Dry Air Flocculation (DAF) followed by centrifugation or filtration. DAF uses floc (inorganic chemical or polyectrolytes) to aggregate algal suspensions into large clumps that can be skimmed off the surface or collected after sedimentation. Centrifugation subjects algal suspension to large gravitation fields that separates water from biomass with up to $95 \%$ efficiency (Greenwell 2011).

DAF is a common technique adopted from water treatment plants where floc agent contamination of collected biomass and high throughput time (too time consuming for the amount of biomass processed) is typical. Centrifugation is considered to be inapplicable as a primary harvesting technique in commercial 
cultivation despite its high efficiency because of its high-energy input requirement - up to $3000 \mathrm{kWh} / \mathrm{ton}$ of algal biomass (Schenk et al 2008). Although, filtration provides some relief from this energy burden, fast build up of biomass eventually demands high-power consumption (Greenwell 2011). Additionally, the very dilute nature of algae culture itself limits how much algae biomass can be concentrated per unit volume of water as concentrations above $15 \%$ gives the algae suspension 'non-Newtonian' characteristics making the liquid very difficult to pump to downstream processing steps (Greenwell 2011).

\subsubsection{Oil Extraction and Recovery}

The algal oil production process culminates in the extraction and recovery of algal oil that becomes feedstock for downstream refining into various transportation fuels. Extraction begins most commonly via mechanical crushing of concentrated cell preparations followed by hexane treatment (hexane is an organic solvent that solubilizes algal oils) that withdraws the oil from the disrupted cellular mixture. However mechanical disruption has not been validated as the best method to prime concentrated cell preparations for rapid extraction of specific cellular targets (Greenwell 2011). Similarly, chemical reagents are not suitable in commercial settings due to the risk of contaminating the chemical integrity of desired products and the consequent difficulty of purifying extracted oil pool (Greenwell 2011).

\subsubsection{Refining}

Unlike the most popular biofuel ethanol, renewable bio-derived jetfuel can be produced from any source of vegetable oils which typically comprise a mixture of saturated and unsaturated fatty acid esters. The composition of the mixture has major implications for the properties of the final jetfuel including its oxidative stability and cold weather properties. In order to ensure compatibility with existing engine technologies, it is important to refine the properties of these biofuels (Vertes et al, 2010) to fit exact standards of the end-use fuel.

Once extracted the algal oil is transported to refineries that have the infrastructural capacity to convert algal oil to AJF via a standard refining technique perfected in crude oil processing called hydroprocessing (Pearlson et al, 2013). The algal oil is cleaned to remove impurities prior to hydrogen gas treatment which converts the oxygen containing compound in the oils (olefins) to paraffins; removal of the oil's natural oxygen content raises the heat of combustion of the fuel and increases it's thermal and oxidative stability (Boeing, 2009). The deoxygenated effluent is cooled and sent to an isomerization unit (Pearlson et al, 2013) where long-chain hydrocarbons are broken to short chained products including jetfuel range hydrocarbons (Boeing, 2009). The isomerized hydrocarbon products are then cooled again before being sent to a separation tower where mixed paraffin gases, carbon dioxide, and excess hydrogen is separated from the liquid products and recycled into 
the hydrotreator. The liquid products are separated into liquefied petroleum gases, naphtha, jetfuel, and diesel streams, and sent to product storage tank farms (Pearlson et al, 2013). Hydroprocessed esters and fatty acids are referred to as Hydroprocessed Esters and Fatty Acids (HEFA) fuels (Pearlson et al, 2013).

Jetfuel made from renewable HEFA feedstock is referred to Synthetic Paraffinic Kerosenes or Bio-SPKs (Boeing, 2009) to differentiate them from Jet A/Jet A-1, Jet Propulsion fuel type 8 (JP-8), or Jet Propulsion fuel type 5 (JP-5) used by commercial aviation, the U.S. Air Force, and the U.S. Navy respectively (ANL, 2012). The main advantages of HEFA fuels are their high cetane values, low aromatic and negligible sulfur content which greatly heighten HEFA's operational performance and negate $\mathrm{SO}_{\mathrm{x}}$ emissions (the prime constituent of smog). Additionally, HEFA fuels are 'drop-in' quality, meaning they are synthetic equivalents of petroleum products and compatible with existing production, storage, distribution, and combustion equipment (Pearlson et al, 2013).

Currently some Bio-SPKs are blended with PJF in varying quantities to compensate for their generally lower energy density and limited existing biofuel production capacity. Most Bio-SPKs must undergo much more extensive and rigorous validation process to ensure that the fuel performs under the operational stress of high altitude flight (Vertes et al, 2010). As of July 2011, ASTM approved a blend of up to 50\% HEFA fuels (with PJF) for further investigation which can potentially allow Bio-SPKs produced from algal oil to be used widely in the future. This follows a similar approval in 2009 that enabled the blending of up to $50 \%$ of fuels produced from another conversion technology Fisher-Tropsch (FT) with conventional jetfuel. Efforts are ongoing to approve additional fuel conversion pathways (ANL, 2012).

\subsection{AJF VALUE CHAIN}

Figure 3a and Figure 3b illustrates the major elements of an ethanol and AJF value chain respectively; the value chains generally encompass farms, storage facilities, biorefineries, blending facilities, retail outlets, and end-consumers (Awudu \& Zhang, 2012) creating a network of value generating stages through which raw biomass is transformed to a variety of marketable products (O'Connell \& Haritos, 2010). Biofuels and Biorefineries operate at the intersection of a range of industries such as forestry, agriculture and waste; they engage actors in transport and infrastructure, and leverage a range of policy regimes at local, national and transnational levels. Due to the broadness of the underlying stakeholder network, a bewildering array of complex interactions inevitably arises between producers, intermediaries and consumers ( $O^{\prime}$ Connell \& Haritos, 2010). However unlike the biodiesel or ethanol value chains, the aviation sector has a relatively streamlined post -refinery distribution system with much fewer supply points and aircrafts compared to the thousands of supply points and millions of vehicles in the road sector. For example, there are 161,768 retail petrol stations in the United States alone compared to only 1,679 airports that handle more than $95 \%$ of the 
world's passengers (SAFUG, 2010). Typically major airports are supplied via one or two fuel pipelines directly from the refinery. Use of the fuel supply infrastructure requires approval from infrastructure owners and agreement with airline operators using the facilities. Part of the economics of Bio-SPKs is driven by access to the existing pipeline infrastructure (CSIRO, 2011). Unlike other alternative jetfuels refined from nonconventional crude like shale oil, Hydroprocessed Esters of Fatty Acids (HEFA) fuels such as AJF provide practical appeal by virtue of meeting or exceeding internationally recognized jetfuel specifications and can be 'dropped-into' existing aircraft engine fuel systems, distribution methods, and storage facilities without significant infrastructure upgrades (SAFUG, 2010). The 2011 ASTM D7566 approval of a 50:50 blend of HEFA aviation fuel batches has been a major step forward in achieving AJF mass adoption (CSIRO, 2011).

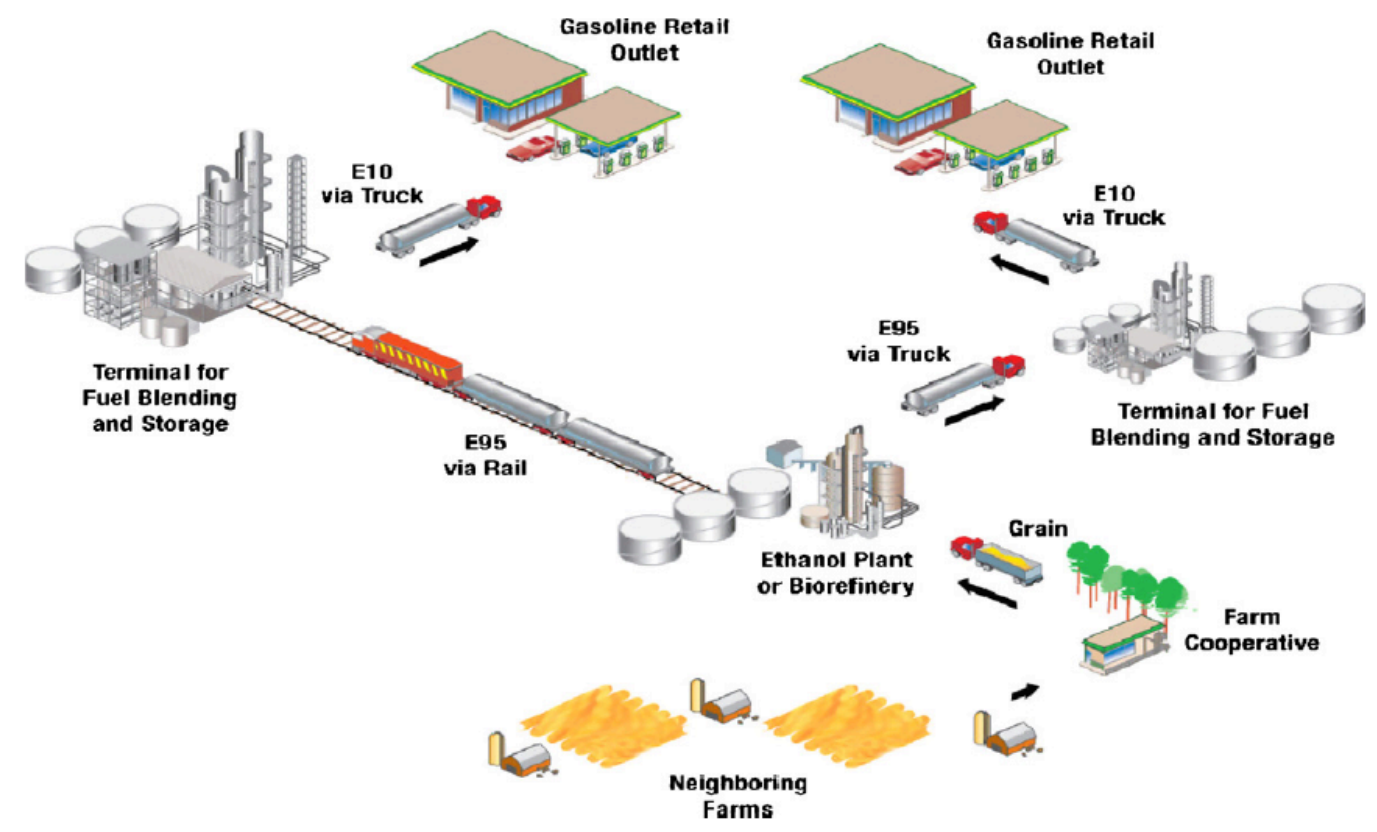

Figure 3a - Corn Ethanol Value Chain (Awudu \& Zhang, 2012). 


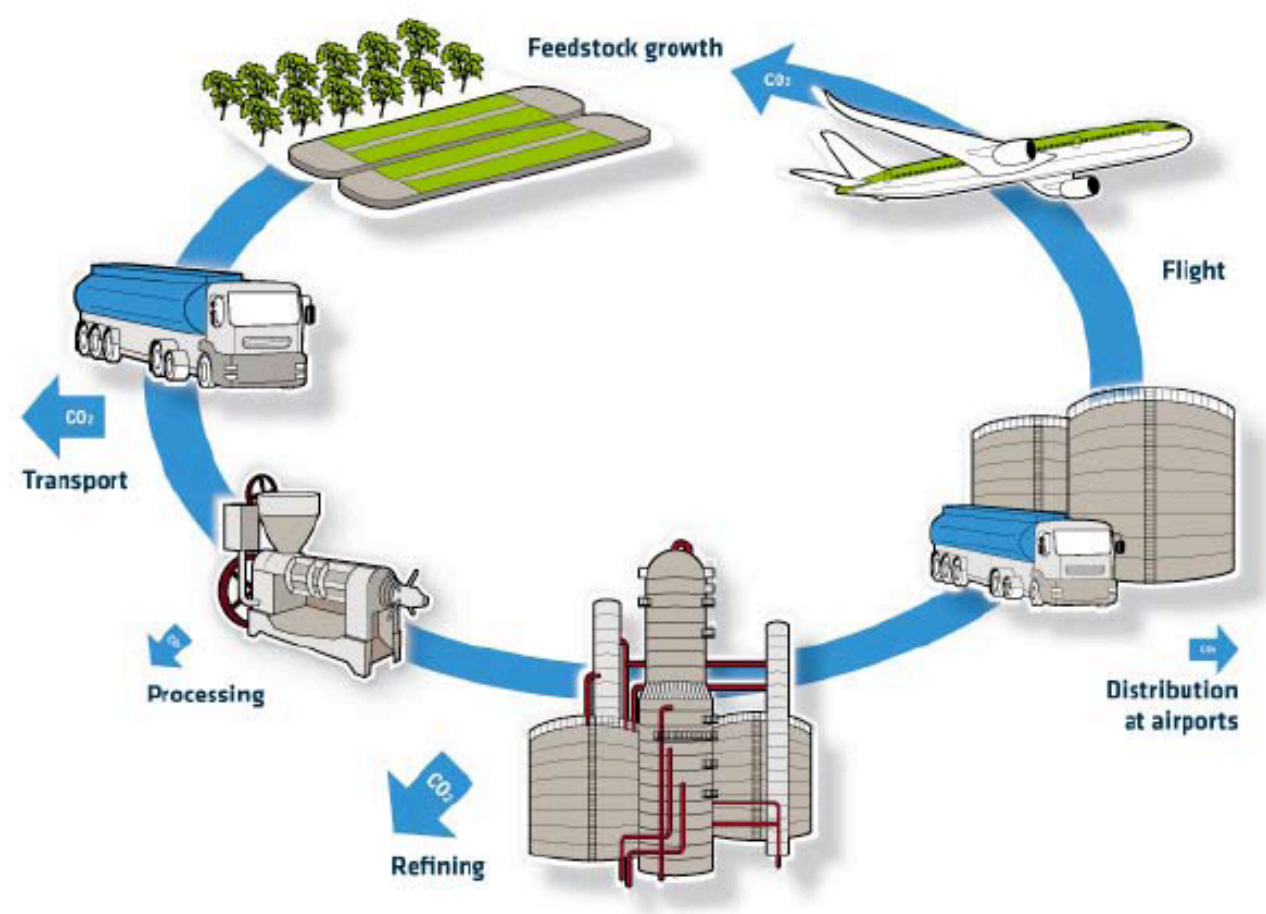

Figure 3b - AJF Value Chain (ATAG 2011).

\subsection{CARBON TAX}

The concept of a carbon tax originated from principles of welfare economics first proposed in 1912 by A.C. Pigou who postulated that the most cost effective way to mitigate the effects of pollution is through a system of tradable quotas (emissions trading) or taxation of pollution intensive goods (Knight et al, 2011). In this sense a carbon tax is a Pigouvian measure aimed at correcting the market failure created by goods and services being priced at a level that does not reflect their true costs imposed on society i.e. the price of petroleum products do not capture the environmental cost imposed on society in terms of their climate change impact, pollution, habitat destruction, health costs etc. A carbon tax levied on a product or service captures it's negative externalities into the market transaction thereby creating a more accurate cost benchmark (Sodero, 2011).

Carbon tax is often used as a policy instrument through which environmental standards are enforced. According to Porter's hypothesis, a carbon tax can impose responsible and efficient use of resources as well as spur emissions-reducing innovations that can in turn cut production costs and boost enterprise competitiveness (Knight et al, 2011); in some notable cases a carbon tax has proved to be effective in accelerating innovation in Renewable Energy Technologies (RETs) and has facilitated their market adoption by leveling the cost gap with fossil fuel energy technologies (Knight et al, 2011). However the wider effectiveness of carbon tax as a mitigation tool is contentious due to the mixed results noted by early- 
adopters like Norway, Sweden and Finland. The overall ineptitude of Norway and Finland's carbon tax is attributed in part to its narrow policy base which provides numerous exemptions to regional emission intensive industries so as to circumvent the feared GDP impact of lower output brought on by penalizing carbon. Alternatively, Sweden's carbon tax is deemed effective in the district-heating sector but has had "no discernible effects" in the transport sector due to the relatively small contribution of the carbon tax to the overall transportation fuel costs (Sodero, 2011).

Having said that the true impacts of a carbon tax policy that targets all sectors of a transportation industry is yet to be seen. This study considers the carbon tax framework implemented by British Colombia (BC) as the basis for the carbon tax level employed in the subsequent economic analysis. BC's carbon tax is unique in that it applies both a relatively high carbon tax and targets the entire transportation sector including aviation (traditionally left out of emissions policies) (Sodero, 2011).

The formulation and implementation of BC's carbon tax was triggered primarily by two phenomena. The first is the acute public concern centered on climate change impacts experienced in BC over the last 50-100 years. During this time B.C. has lost roughly $50 \%$ of its snow covering while its total annual precipitation has increased by about $20 \%$. The increasingly erratic weather patterns are understood to be consistent with the Intergovernmental Panel on Climate Change (IPCC) findings (BC, 2008). Concurrently, warmer winters also contributed to the mountain pine beetle epidemic that has so far decimated more than 13 million hectares of pristine pine forest - an area equivalent to four times the size of Vancouver Island. The beetle's numbers have historically been controlled by cold winters but warmer weather is now directly linked to their devastating spread (BC, 2008).

Climate change and pine beetles moved to the foreground of political and public dialogue in BC particularly between 2001 and 2006 (Sodero, 2011). In September 2006, the Republican Governor of California Arnold Schwarzenegger approved the Global Warming Solutions Act committing California to achieve 1990 greenhouse gas emission levels by 2020, and to this end permitted the use of both market-based mechanisms and regulation. The Act received accolades for its pioneering foresight, prompting then BC Premier Campbell to coordinate a period of collaboration between California and BC on climate change measures (Sodero, 2011). In November 2007, BC's Greenhouse Gas Reduction Targets Act entrenched in law its commitment to reduce GHG emissions by $33 \%$ by 2020 , and by $80 \%$ by 2050 compared to 2007 levels. The carbon tax is one of few instruments to be used to reach this end (BC, 2008).

BC's carbon tax is applied and collected in essentially the same way that motor fuel taxes have been collected at the point of production or distribution, which minimizes the governments' cost of administration to government and the compliance cost to those collecting the tax on government's behalf (BC Ministry of Finance, 2013). The tax rate started low in 2007 and increased gradually by $\$ 5$ every year, reaching a final 
price of $\$ 30$ per ton of $\mathrm{CO}_{2}$ equivalent $\left(\mathrm{tCO}_{2} \mathrm{e}\right)$ emissions by 2012; starting low gave consumers time to make adjustments from decisions made prior to the announcement of the tax (BC, 2008). The tax has the broadest possible base; all emissions from fossil fuel combustion in B.C. noted in Environment Canada's National Inventory Report is taxed with no exemptions made except those required for efficient administration and eventual integration with other climate action policies planned for the future (BC, 2008). Unlike major Scandinavian countries, BC's carbon tax is used as an emission reduction tool and not a revenue generation tool (Sodero, 2011). The tax mitigates fears of any negative impacts on economic activity and competitive advantage of businesses (Lin \& Li, 2011) by functioning under a revenue neutral framework where all carbon tax revenue is recycled through corporate and individual income tax reductions. The BC government is legally bound to present an annual plan to the legislature demonstrating how all of the carbon tax revenue has been returned to taxpayers through tax reductions $(\mathrm{BC}, 2008)$.

The carbon tax is a key element in BC's emissions reductions strategy but is not expected to meet to meet the targets alone. In time the tax framework will be integrated with collaborative programs with Quebec and California. To avoid unfairness and what can effectively become double taxation, the carbon tax and complementary measures such as the "cap and trade" system will be assimilated as these other measures are designed and implemented (BC, 2008). BC's carbon tax is North America's first broad based revenue neutral carbon tax (BC, 2012). In time the carbon tax is expected to send a price signal that will elicit a powerful market response across the entire economy and result in reduced emissions (BC, 2008).

Although its has been 5 years since the tax was implemented, at this moment there are several factors that prevent a definitive assessment of the tax's efficacy, a major reason being that emissions data is only available up to 2010 covering only three full years of carbon tax implementation; emission levels during the period were also substantially affected by economic slowdown brought on by the global economic recession, the manifestations of which cannot be distinguished from emissions reductions triggered solely by the tax. Additionally, the gradual rise in the carbon tax traded some emissions reduction effectiveness in the short term for a smoother transition to higher rates over time with the intention that mitigation effectiveness would increase as rates increased, and as stakeholders had time to adjust their habits in response to the carbon tax (BC, 2012).

\subsection{COMMERCIALIZATION OF INNOVATION}

To produce AJF at commercial levels and an economically feasible cost, massive investment is required in algal feedstock Research and Development (R\&D (and associated conversion technology) and downstream biofuel value chain (O'Connell \& Haritos, 2010). Literature suggests that powering commercialization of new energy technologies through mobilization of large investments is difficult as incumbent energy suppliers and 
often adopters of RETs - utilities, oil and gas companies, pipeline companies - have already employed capitalintensive assets to produce and distribute energy derived from fossil fuels and uranium (nuclear power). The strategic focus of these entities is to maximize the return on their embedded capital as large investments require large returns; incumbent energy technologies provide relatively low risk returns on investment due to tried and tested technology and a long history of familiarity (Walsh, 2012).

Risk, as defined by the uncertainty of a profitable outcome, is a critical component of investment decisions. Risks can be a manifestation of various characteristics innate to the innovation in question and its contextual market conditions. Sources of technological risks for AJF are (but not limited to) i) production risk (growth medium/system health, quantity of biomass, oil content and co-product yields) ii) input prices $\left(\mathrm{CO}_{2}\right.$, labor, nutrients, water, electricity, construction, and maintenance) iii) output prices (algal oil and co-product prices) iv) resource availability (water, $\mathrm{CO} 2$, and land) v) and climate and location (days of operation, net evaporation, and temperature) (Richardson et al, 2012).

Investors also face technology independent risks when investing in new technologies. The "adoption hurdle", presented by the uncertainty of whether a new technology will be adopted by end-use markets, impedes the ability of investors to predict with confidence the return on their investment, leading them to under-invest in new ideas and instead deploy capital into to less risky projects where profitability is more predictable (Knight et al, 2011). The second hurdle is the "spillover hurdle". Even if markets adopt the new technology, developers and investors may not be fully compensated due to positive externalities derived from R\&D investment in that new idea. "The spill-over effects of this investment are captured by competitors who indirectly benefit by replicating the intellectual property and take advantage of new technological know-how. The presence of this incentive asymmetry creates a second barrier to private investment in new technology" (Knight et al, 2011, pg. 7).

The commercialization environment for an innovation can be defined by prevalent technology-push or demand-pull drivers (Walsh, 2012). Technology-push occurs when an emerging technology induces a market demand, as opposed to a demand-pull scenario where the market calls for an innovative product to satisfy a market need. "In other words, demand-pull drives innovation to seek to solve a market problem that cannot be solved with existing technology whereas technology-push results in the adoption of an innovation because the technical capabilities of that innovation establish a demand in the market, regardless of whether or not an initial demand existed "(Walsh, 2012, pg. 34). In this sense, despite the disincentive for investments in renewable solutions, investors are drawn by the market-pull conditions created by the lack of short-term renewable alternatives to PJF. AJF has the potential to meet all of aviation's criteria for a sustainable alternative but being a nascent technology it embodies many risks that disallows straightforward projections about the profitability of any potential investments made in developing or commercializing it. Hence 
evaluating AJF on its merits, potential profitability and risks becomes an essential exercise to determine whether the technology can bear fruit for investors.

This study culminates in an economic assessment of AJF from a project valuation perspective that includes the inclusion of carbon reduction valuation. The financial feasibility of a hypothetical AJF value chain depends on its Capital Expenses (CAPEX), Operating Expenses (OPEX), and expected annual cash flows over its operational life. In addition, the valuation model takes into account risk in form of the Weighted Average Cost of Capital (WACC) characteristic of the aviation industry (Richardson et al, 2012). Details of the valuation model used and its entailing assumptions are covered in the following methodology section. 


\section{METHODOLOGY}

\subsection{NET PRESENT VALUE (NPV)}

Investigating the commercializing potential of AJF technology is essentially an exercise in evaluating the profitability of an investment in a hypothetical AJF value chain. To do this, this study supposes a simple scenario - the aviation industry is a single entity that financially commits to a linear AJF value chain and investor expectation of some return on the investment. The investor operates the algal plant, produces oil, and oversees its transformation and distribution in the jetfuel market. The net revenue generated from sale of AJF returns to the investor as profit. Not only must the AJF technology achieve a surplus from operations to cover its operation expenses, but it must also provide a surplus to meet the investment's expected return. The approach follows the simplified conceptual framework used by Shiho et al (Shiho et al, 2012). In order to estimate the expected profitability of this venture, or lack thereof, a Net Present Value (NPV) analysis is used. NPV sums the newly added capital value of an investment generated through business operations, and when this value becomes positive, investors receive positive indication that their capital commitment might entail promising prospects (Shiho et al, 2012).

NPV derives from the concept of Discounted Cash Flow (DCF), a fundamental valuation methodology broadly employed by investment bankers, corporate officers, university professors and investors. It rests on the notion of time value of money, which in simple terms describes the future uncertainty of investments made in the present time i.e. time value of money captures the value of future transactions that result from a capital investment made in the present, describing potential monetary transactions in present day terms. In this way, the value of an investment can be estimated from the Present Value (PV) of its projected Cash Flows (CF), or the revenues and costs generated over the lifetime of the business venture as a result of the investment. Future CFs discounted in today's terms are called DCFs (Rosenbaum \& Pearl, 2009). The value of a target investment described by it's projected DCFs is considered to be the investment's intrinsic value as opposed to its market value, which is the value ascribed by the market at a given point in time. In practical terms DCFs serve as an important analogue to market-based valuation techniques which are often subject to external market forces that can distort the true valuation of the target investment (Rosenbaum \& Pearl, 2009). DCFs are informed by assumptions and judgments immediate to the target investment like expected financial performance including sales growth rates, profit margins and capital expenditures requirements (Rosenbaum \& Pearl, 2009).

Conventionally to calculate DCFs, the lifetime Free Cash Flows (FCFs) are first determined. FCFs are the cash generated by a project after all OPEX, associated taxes, CAPEX and working capital are committed, but prior 
to making any interest payments (due from institutional debt) (Rosenbaum \& Pearl, 2009). FCF is a practical measure of a venture's projected financial performance because it factors out tax ramifications and depreciation of capital assets. But FCF calculation warrants a deeper study of market conditions and regional tax codes which in addition to being outside the scope of this study, is also difficult to conduct in the absence of any commercial scale AJF operations that could provide cash flow benchmarks to inform the financial assumptions of the hypothetical AJF value chain. Instead, this study derives the DCFs from the project's Earnings Before Interest, Taxes, Depreciation and Amortization (EBITDA) (Rosenbaum \& Pearl, 2009) to separate the final NPV from consideration for any jurisdiction-specific treatment of corporate activity.

\subsection{EBITDA Formula}

\section{i) The EBITDA formula (Sun et al, 2011) used is:}

EBITDA = Revenue (from sale of AJF) + Revenue (monetized emissions benefit from AJF) - Cost of Production of AJF

* Cost of Production includes CAPEX factors (cost of land, equipment, facilities, and indirect expenditures) and OPEX factors (labor, maintenance cost, raw material cost, and utility cost)

Each yearly EBITDA is discounted to PV to derive yearly DCF over the entire valuation period. Total EBITDA is calculated from all years in the valuation period.

\section{ii) The formula for PV (Fabozzi, 2009) is:}

$\mathrm{PV}=(\mathrm{CF}) /\left[(1+\mathrm{i})^{\mathrm{N}}\right]$

Here ' $i$ ' is the Discount Factor (DF). The DF is the numerical representation of the risk facing the investment's projected returns (Fabozzi, 2009). Profitability may vary for companies within a given sector depending on a multitude of factors including management, brand, customer base, operational focus, product mix, sales/marketing strategy, scale, and technology. A venture's growth profile can vary significantly from that of its sector peers and so different companies, industries and investment scenarios can be characterized by different DFs (Rosenbaum \& Pearl, 2009). The DF used in aviation's case is called the Weighted Average Cost of Capital (WACC). Airlines can be described by varying costs of capital due to differences in operational challenges, profitability, risk profiles and tax ramifications. The WACC employed in this study is an aviation industry-wide estimation derived from the 'sum of its parts' (Rosenbaum \& Pearl, 2009) and quoted by the IATA in its financial forecast (IATA Financial Forecast, 2012). The WACC is subject to the capital structure embodied by the investor entity i.e. the capital contributed by its debt providers (institutional lenders) and equity providers (shareholders). The Aviation industry has a WACC of 7\% (IATA Financial Forecast, 2012) meaning that every year, the sector must contribute a minimum of at least $7 \%$ of its earnings to meet 
commitments to its debt providers and equity holders. Hence $7 \%$ represents the benchmark for success for an investment made by the aviation industry regardless of the subject of investment (Rosenbaum \& Pearl, 2009).

The second element needed to convert a future CF into a DCF is the valuation period captured by ' $\mathrm{N}$ ' representing every year in the valuation period. It is vital to project CFs to a point in the future where the investment's financial performance reaches a steady state signified by predictable and repeated CFs. For mature industries, five years is often sufficient to allow a company to reach its steady state. However for businesses in sectors with long-term contracted revenue streams such as in natural resources, satellite communications, or utilities, and early stage innovations like the algal biofuel pathway, which does not yet have a clear roadmap to commercialization, it is more appropriate to consider a longer-term valuation period of ten years (Rosenbaum \& Pearl, 2009). Additionally, ten years is also approximately the amount of time the IEA estimates algal biofuels technologies will take to evolve from pilot and demonstration scale projects to large-scale commercial farms (IEA 2011).

The last two elements requisite of an NPV analysis is the Upfront Capital Cost (UCC) and the Terminal Value (TV). The UCC refers to the capital investment made to put 'steel in the ground' or to buy, build and develop the infrastructure assets (such as ponds, pipelines, buildings etc.) before algal cultivation can even begin. This is a negative CF that occurs in year 0 (Shiho et al, 2012; Rosenbaum \& Pearl, 2009). Because it is also infeasible to extrapolate an investment's future CFs indefinitely, a TV is used to capture the value of the investment beyond the projection period. The TV is critical because it typically accounts for a substantial portion of the value generated by an investment - sometimes as much as $75 \%$ or more. TV is calculated from the venture's EBITDA in the final year of the projection period (Rosenbaum \& Pearl, 2009).

\section{iii) The formula for TV (Fabozzi, 2009) is:}

$\mathrm{TV}=\left(10^{\text {th }} \mathrm{EBITDA}\right) / \mathrm{DF}$

\subsubsection{EBITDA Inputs}

The following sections articulate the assumptions and justifications used to determine the Cost of Production (COP) of the algal oil, the Cost of Refining (COR) the algal oil into AJF, the revenue stream generated from the sale of AJF, and finally the revenue stream generated from the emissions benefit of using low carbon intensity AJF.

\section{i) Cost of Production (COP)}

In the absence of large-scale algal biofuel operations, most cost estimations for per unit algal oil are based to some extent on productivity and financial performance of non-fuel specific algal cultivation for specialty 
chemicals such as betacarotene and other non-oil products. These facilities are not optimized for oil production and therefore produce relatively little feedstock at high cost. The most widely recognized cost performance model of a hypothetical commercial algal oil production facility comes from a seminal report authored by Benemann and Oswald in 1996 (compiled for the National Energy Technology Laboratory (NREL)) which covers a comprehensive assessment of the research, technologies, commercial applications, and economics of open pond algal biomass production for biodiesel production. Funded by the US DoE's Aquatic Species Program, Benemann and Oswald's report is one of most cited references on cost estimations for algae cultivation for carbon sequestration and has become the cornerstone for a lot of recent cutting edge technoeconomic modeling (Sun et al, 2011). Having said that, there is also a wide range of cost estimations reported for the per unit cost of algal oil with some extremes varying by up to two orders of magnitude. Table 1, compiled by US DoE's Office of Energy Efficiency and Renewable Energy (EERE), highlights the multitude of assumptions used in modeling algal oil costs (in \$/gallon) using open ponds and photobioreactors (alternative novel cultivation technologies that are much newer than open pond cultivation and perform even less competitively) (Sun et al, 2011).

\begin{tabular}{|c|c|c|c|c|c|c|}
\hline Source & Scenario & Cultivation & $\begin{array}{l}\text { Cost } \\
\left(\text { USD gal }^{-1}\right)\end{array}$ & $\begin{array}{l}\text { Lipid yield } \\
\text { (wt.\% of dry mass) }\end{array}$ & $\begin{array}{l}\text { Areal Dry Algae Mass } \\
\text { Yield }\left(\mathrm{gm} \mathrm{m}^{-1} \text { day }^{-1}\right)\end{array}$ & $\begin{array}{l}\text { Loan Period } \\
\text { (yrs) }\end{array}$ \\
\hline Benemann & Baseline & Open pond & $\$ 1.7$ & $50 \%$ & 30 & 5 \\
\hline Benemann & Maximum growth & Open pond & $\$ 1.2$ & $50 \%$ & 60 & 5 \\
\hline NREL & Current & Open pond & $\$ 10.6$ & $25 \%$ & 20 & 15 \\
\hline NREL. & Aggressive & Open pond & $\$ 3.5$ & $50 \%$ & 50 & 15 \\
\hline NREL & Maximum growth & Open pond & $\$ 2.4$ & $60 \%$ & 60 & 15 \\
\hline NMSU & Current, 1 acre & Open pond & $\$ 38.7$ & $35 \%$ & 35 & 20 \\
\hline NMSU & Highest yield, 1 acre & Open pond & $\$ 13.9$ & $60 \%$ & 58 & 20 \\
\hline NMSU & Current, 2000 hectare & Open pond & $\$ 25.2$ & $35 \%$ & 35 & 20 \\
\hline NMSU & Highest yield, 2000 hectare & Open pond & $\$ 9.7$ & $60 \%$ & 58 & 20 \\
\hline Solix & Current & Hybrid & $\$ 31.8$ & $16-47 \%$ & $0-25$ & unknown \\
\hline Solix & Phase I & Hybrid & $\$ 2.6$ & $16-47 \%$ & $30-40$ & unknown \\
\hline Solix & Phase II & Hybrid & $\$ 0.9$ & $16-47 \%$ & $30-40$ & unknown \\
\hline Seambiotic/IEC, Israel & Best yield & Open pond & $\$ 24.9$ & $35 \%^{a}$ & 20 & unknown \\
\hline Sandia & Current & Open & $\$ 15.7$ & $35 \%$ & 30 & 10 \\
\hline Sandia & Current & PBR & $\$ 33.2$ & $35 \%$ & 30 & 10 \\
\hline Bayer Tech Services & Optimistic & PBR & $\$ 14.3$ & $33 \%$ & 52 & 10 \\
\hline General Atomic & Low & Open/hybrid & $\$ 20.0$ & unknown & unknown & unknown \\
\hline General Atomic & High & Open/hybrid & $\$ 32.8$ & unknown & unknown & unknown \\
\hline California Polytech, Pomona & Waste treatment + digester & Open pond & $\$ 16.8$ & $25 \%$ & 20 & 8 \\
\hline Tapie \& Bernard & Tubes on ground & PBR & $\$ 40.6$ & $35 \%$ & 20 & 5 \\
\hline Tapie \& Bernard & Double tubular bioreactor & PBR & $\$ 43.1$ & $35 \%$ & 20 & 5 \\
\hline
\end{tabular}

Table 1 - US DoE EERE evaluation of algae technology. Current or baseline scenario refers to the estimated productivity and oil yield in 2008 (Source: Sun et al, 2011).

The wide disparity in reported COP naturally makes any baseline cost assumptions for the present analysis very difficult. To overcome this, a comprehensive baseline harmonization study published by the Argonne National Laboratory (ANL), a prominent research institution at the forefront of algal biofuel research and modeling, is used for cost estimations. ANL designed the study with the goal to harmonize existing disparate methodologies for COP estimations into a unified model that is consistent and verifiable. ANL normalized the differences between the three dominant types of feasibility studies commonly undertaken in the algal biofuel arena - Technoeconomic Assessment (TEA), Lifecycle Assessment (LCA) and Resource Allocation (RA). TEA, 
LCA and RA study the major determinants of sustainable algal cultivation for fuel; TEAs elucidate the economic performance of algal production regime by employing a particular layout of technology and is strongly influenced by CAPEX and OPEX; the LCA approach is concerned primarily with operating variables such as yields, material, energy or emissions input/output inventories which, in contrast to TEA, scale with level of operations (Argonne National Laboratory, Pacific Northwest National Laboratory, National Renewable Energy Laboratory, 2012); the RA models algae productivity and the associated water footprint (ANL et al, 2012).

Prior to harmonization the TEA, LCA and RA models differed in a number of critical parameters including the oil content of the algal strain, the productivity ( $\mathrm{g} / \mathrm{m}^{2} /$ day) of open pond system and facility scales, all of which naturally resulted in different algal oil yields. For example, the LCA assumed a farm with 4700 hectare (ha) of ponds, RA assumed 405 ha, while TEA was based on 1955 ha of ponds. In contrast the harmonized model was created around groupings of 10 RA-based 405 ha unit farms totaling 4050 hectares of total pond area (4850 ha total facility footprint, including all processing operations) producing a maximum of 39.31Million Liter per Year (MLY) of algal oil. Each individual pond was assumed to be cultivating algae with $25 \%$ oil content, producing oil at a density of $920 \mathrm{~kg} / \mathrm{m}^{3}$ and have a productivity of $25 \mathrm{~g} / \mathrm{m}^{2} /$ day (ANL et al, 2012). Site selection for the 4850 ha facility was guided by Pacific Northwest National Laboratory's (PNNL) Biomass Assessment Tool that identified suitable land areas within the conterminous United States. The vast majority of the farm's water footprint was associated with making up for evaporative losses from the ponds with a small amount of water lost with sludge from the Anaerobic Digester (AD) (ANL et al, 2012). Makeup water came from freshwater resources; $5 \%$ of the mean annual flow for each underlying watershed was drawn for algae ponds - a number based on the amount of water that the US Environmental Protection Agency allows power plants to withdraw. The study conceded that future work will have to include the possibilities of using saltwater and wastewater as growth mediums to address freshwater scarcity (ANL et al, 2012).

The harmonization study considers total production 18.9 Billion Liters per Year (BLY) coming from multiple 39.31 MLY facilities to calculate the final per unit algal oil COP. The 18.9 BLY quota is derived from the minimum volume of algal biofuels required to meet the 36-billion-gallons-by-2022 EISA mandate for advanced biofuels. However the final COP quoted cannot be imported directly as a cost input for the NPV calculation for two reasons. Firstly, the harmonization model assumes that all algal oil produced is feedstock for the production of biodiesel for road transportation only and not aviation fuel. Secondly, the final COP value is a result of producing the full 18.9 BLY, offering significant scale for a relatively low final COP (ANL et al, 2012). The first limitation is circumvented by considering the COP for the algal oil and not the final enduse product (biodiesel) since raw algal oil is feedstock for both biodiesel and AJF. The second limitation is overcome by the fact that although it is assumed that all the algal oil required to meet the 18.9 BLY EISA quota is produced, only one 39.31 MLY facility is used as the subject of the NPV valuation i.e. sufficient numbers of investors own enough facilities to produce all 18.9 BLY, the aviation industry is one such investor 
committing to one 39.31 MLY facility. In this way, it will be valid to the use the harmonized algal oil COP for the NPV calculation.

The algal oil COP is $\$ 2.25 /$ l. This is the manufacturing price for algal oil accounting for the CAPEX and OPEX, and not the Minimum Selling Price (MSP) that normally includes a markup of a 10\% profit (ANL et al, 2012). The COP is used instead of the MSP to avoid speculation of what the profit margin of the final product will be. The COP represents the lowest NPV that can be expected from producing algal oil at a facility; a MSP at whatever level will only increase the NPV valuation.

The final figure extracted from the harmonization study is the UCC. The total upfront investment required for every 39.31 MLY facility inclusive of CAPEX and OPEX is $\$ 947.30$ million. As indicated, this is a negative CF item that occurs at the time of construction in year 0 to allow algal oil to be produced from year 1 (ANL et al, 2012).

\section{iii) Cost of Refining (COR)}

COR represents the costs incurred to convert the raw algal oil feedstock to AJF using hydroprocessing. The costs for hydroprocessing is derived from a MIT modeling study that assessed the per unit cost of Biologically derived Synthetic Paraffinic Kerosenes (Bio-SPKs) derived from soybeans, a HEFA analogue that can simulate the cost performance expected from algal oil refining. Vegetable oils including algal oil contain relatively short-chained hydrocarbons that can be readily broken to shorter diesel range molecules. As Figure 4 shows, since jetfuel hydrocarbons are smaller, refiners can choose to produce more jetfuel by breaking the diesel chains down to the jet range, allowing the flexibility to vary production to match the market demands for diesel or jetfuel. MIT's model refined HEFA Jetfuel Price (JFP) under two scenarios (i) maximum distillate (diesel) production, and (ii) maximum jet production (Pearlson et al, 2013). In reality, because longer diesel range products have higher market value compared to jetfuel derivatives, refiners tend to always produce some diesel range product even under the maximum jet fuel production scenario (Pearlson et al, 2013). 




Figure 4 - Typical Distillation Ranges (Degrees C) and Carbon-Number Ranges for Fuels (Source: RAND, 2009).

For the purpose of this study, the ideal maximum jetfuel scenario is chosen in conjunction with the most optimal CAPEX and OPEX parameters for a new HEFA refining facility. The model biorefinery is a 378 MLY facility (largest facility option to allow for economies of scale) built on brownfields (discarded industrial land or deactivated refineries with already built infrastructure), and uses hydrogen bought from a third party (to spare CAPEX from building hydrogen generating infrastructure). The final COR is $\$ 0.34 /$ liter (Pearlson et al, 2013).

The summation of COP and COR, total of $\$ 2.59 / 1$, is the cost incurred by the producer (investor) to cultivate, harvest, extract and refine the algae biomass into AJF and $\$ 2.59 / \mathrm{l}$ is the value that is used to calculate the total cost of producing at steady rate of 39.31 MLY for the ten-year valuation period.

\section{iv) Revenue from AJF}

The main source of revenue and positive CF is the revenue generated from the sale of the refined end product AJF. AJF is a drop-in fuel that is fully substitutable with conventional PJF and can therefore be expected to fetch the full price of its fossil fuel counterpart (Boeing, 2009). As of February 2013, the average Jetfuel Price (JFP) for 2013 reported by the IATA was $\$ 132.80$ /barrel (IATA, Feb 2013) translating to $\$ 0.84 /$ l. It is assumed that all 39.31 million liters produced at a steady rate each year for the ten-year valuation period is sold at $\$ 0.84 / 1$. 


\section{v) Revenue from Emission Benefit}

Since AJF is posited as a sustainable alternative to PJF, its proponents seek at a minimum to ensure that the overall AJF value chain leads to carbon neutrality $\left(\mathrm{CO}_{2} \mathrm{e}\right)$ compared to PJF (Grierson \& Strezov, 2012)). This study is premised on the assumption that $\mathrm{AJF}$ in providing significant $\mathrm{CO}_{2} \mathrm{e}$ savings, can also provide monetary savings if AJF is produced and consumed in a jurisdiction where a carbon tax is applicable. Because PJF combustion releases far more lifecycle $\mathrm{CO}_{2}$ for every unit of energy extracted from it, a carbon tax will signal a monetary incentive for aviation to opt for AJF instead of PJF to avoid the emission penalty. For the purpose of a NPV analysis, the emissions savings is treated as a positive cash flow.

The LCA data for AJF is leveraged to establish its lifecycle emissions balance. LCA is a tool within the broad discipline of lifecycle management, an approach commonly used as a means to benchmark and compare designs, processes and systems, with a view to continuous improvement around set criterion. Based on standardized methods published by the International Standards Organization, it can provide valuable insight into the aggregate efficiency and impact of discrete energy and material flows that are relevant to processing and manufacture of a product across its various lifecycle stages (Grierson \& Strezov, 2012)). While TEAs primarily address the economic feasibility of the process overall, LCAs depending on the input/outputs may or may not be used to describe the environmental impacts of a product or process; LCA is based on relevant assumptions of productivity, as well as material and energy flows (Grierson \& Strezov, 2012)).

A LCA of aviation fuel using $\mathrm{CO}_{2}$ equivalent $\left(\mathrm{CO}_{2} \mathrm{e}\right)$ flows is called a Well-To-Wake (WTWa) assessment. As illustrated in Figure 5, a WTWa encompasses two stages. In the first step, the Well-to-Pump (WTP) emissions of $\mathrm{CO}_{2}$, methane $\left(\mathrm{CH}_{4}\right)$, and Nitrous Oxide $\left(\mathrm{N}_{2} \mathrm{O}\right)$ associated with delivering 1 Mega Joule (MJ) of energy to the fuel tank of an aircraft is measured. Included in WTP accounting are emissions associated with obtaining the raw fuel (extracting crude oil or cultivating and harvesting biomass), transporting it, processing the raw fuel to jetfuel, and delivering the jetfuel to aircraft fuel tanks. Estimated emissions of $\mathrm{CH}_{4}$ and $\mathrm{N}_{2} \mathrm{O}$ are converted to $\mathrm{CO}_{2}$ equivalents using their relative global-warming potential (RAND, 2009). In the second stage, the Pump-To-Wheel or in the case of aviation Pump-To-Wake (PTWa), accounts for the amount of $\mathrm{CO}_{2}$ released during the combustion of $1 \mathrm{MJ}$ equivalent of fuel in the aircraft turbine engines. Calculating $\mathrm{CO}_{2}$ combustion emissions is straightforward and depends only on the chemical composition of the fuel (chemical integrity of jetfuel is highly standardized), allowing easy estimation of how much more or less $\mathrm{CO}_{2} \mathrm{e}$ emission is released with the production and consumption of $1 \mathrm{MJ}$ of the AJF as compared to PJF (RAND, 2009). 




\section{Well-to-Pump (WTP)}

Pump-to-Wheels

\section{Well-to-Wheels (WTW)}

Figure 5 - WTP, PTW, WTW Definitions for Algae Biofuel (Source: ANL, Dec 2011).

LCAs identify environmental risks or 'hotspots' within a products' lifecycle to guide benchmarking and communication of performance parameters more credibly by virtue of offering a common verifiable methodology by which footprints of different products, systems or processes can be effectively compared to their relevant alternatives (Grierson \& Strezov, 2012)). Having said that, LCA models can also vary widely in their underlying assumptions and boundary conditions which creates discrepancies in the overall balance for the metric being measured. The production of algal biofuels present further complications for LCA benchmarking due to the multitude of possible production schematics and process options (ANL, Dec 2011) currently being employed. Adequately assessing the energy and emission flows of the algal oil production stage is difficult in the absence of commercial operations making lab scale data the only source to extrapolate the environmental impact of industrial scale production of AJF. Algal cultivation, harvest and process technologies for fuel derivatives are mostly immature leaving most studies presenting only a prospective LCA, even more so for a hypothetical facility (Grierson \& Strezov, 2012)). And even amongst these studies, the LCA models vary in their considerations for emission flows associated with building infrastructure (manufacturing and transport of concrete, pipes, equipment etc.) and/or recycling of growth nutrients for algal cultivation. The task of capturing all energy or emission flows across all material supply chains is naturally daunting, therefore necessitating boundary conditions under which the energy or emission transactions can be counted. Due to the current lack of an universal methodology for emissions accounting, models built in isolation inadvertently differ in overall emission balance.

The need for an universal LCA framework to quantify the environmental impact of advanced biofuels has given rise to one specific model that has been valorized by both the aviation industry and academia as the standard for transportation fuels and vehicle systems. The Greenhouse Gases, Regulated Emissions and Energy use in Transportation (GREET) is employed to elucidate the lifecycle balance of PJF and AJF in this study. 


\section{Greenhouse Gases, Regulated Emissions and Energy use in Transportation (GREET)}

GREET is a publicly available LCA tool to consistently examine lifecycle energy and emissions flows in over 85 vehicle systems (including gasoline, diesel, plug-in hybrid, battery-powered, fuel-cell vehicles etc.) and fuel systems (including gasoline, diesel, hydrogen, natural gas-based fuels, electricity, etc.). GREET measures emissions impact by recording $\mathrm{CO}_{2}, \mathrm{CH}_{4}$, and $\mathrm{N}_{2} \mathrm{O}$ transactions as well as common criteria pollutant emissions (VOC, $\mathrm{CO}, \mathrm{NO}_{\mathrm{x}}, \mathrm{SO}_{\mathrm{x}}, \mathrm{PM}_{2.5}$, and $\mathrm{PM}_{10}$ ). It was designed in partnership with prominent government research institutions like the NREL and PNNL, US DoE and US EPA, industry stakeholders (energy companies, biofuels producers, technology developers, vehicle manufacturers) and NGOs (ARGONNE APRIL 2011). The tool has been instrumental in developing key federal renewable energy policies such as US EPA's Renewable Fuels Standard 2 (RFS 2), California Air Resources Board's Low Carbon Fuel Standard (CARB LCFS), and is regularly cited in congressional testimonies and reports encompassing GHG impact of transportation. In each of these cases, GREET was used to weigh biofuel technology options, improve process energy efficiencies to achieve low carbon footprint and steer educated public debate around biofuel technologies and policies (ARGONNE APRIL 2011).

GREET addresses the major challenge faced by other LCA methodologies by bringing together multiple process scenarios differing in species selection, cultivation, intermediate constituents, conversion processes as well as end-use fuels and markets. GREET effectively harmonizes data collection, boundary definition and/or assessment methods that otherwise vary due to the lack of an universal methodology (Grierson \& Strezov, 2012)).

The GREET platform applied to AJF consists of two interconnected excel sheets. The Algal Process Description (APD) defines the overall boundary for the production process pathway (Figure 6) and describes the intermediate technologies used for cultivation, harvest and extraction as well as their performance efficiencies. As Figure 7 illustrates, the default production schematic uses open cultivation, DAF and centrifuge to harvest and dewater biomass respectively, followed by mechanical grinding and solvent (hexane) treatment to extract the desired algal oil. APD also accounts for direct energy inputs (and associated emissions) required by the core technologies, the peripheral equipment such as the pumps and blowers delivering $\mathrm{CO}_{2}$ to the ponds, and the main power source for the facility. The overall emissions balance of the production pathway and the facility is intimately tied to the nutrient balance and the fate of the Lipid Extracted Algae (LEA) biomass. The default APD pathway passes the LEA into an Anaerobic Digester (AD) that produces biogas to generate electricity downstream for the facility, while combustion $\mathrm{CO}_{2}$ is recycles back into the cultivation ponds to feed new biomass. Concurrently, AD residue containing vital nitrogen and phosphorus nutrients are also passed into the cultivation ponds to drive the growth of newer batches of algae. Recycling of nutrients and generating electricity on-site mitigates the emission footprint of the algal oil production facility as less power is drawn from the grid (which may or may not be produced from carbon 
intensive fossil fuels) and less energy is expensed to manufacture growth limiting nutrients from fossil fuel derived fertilizer (ANL, Dec 2011).

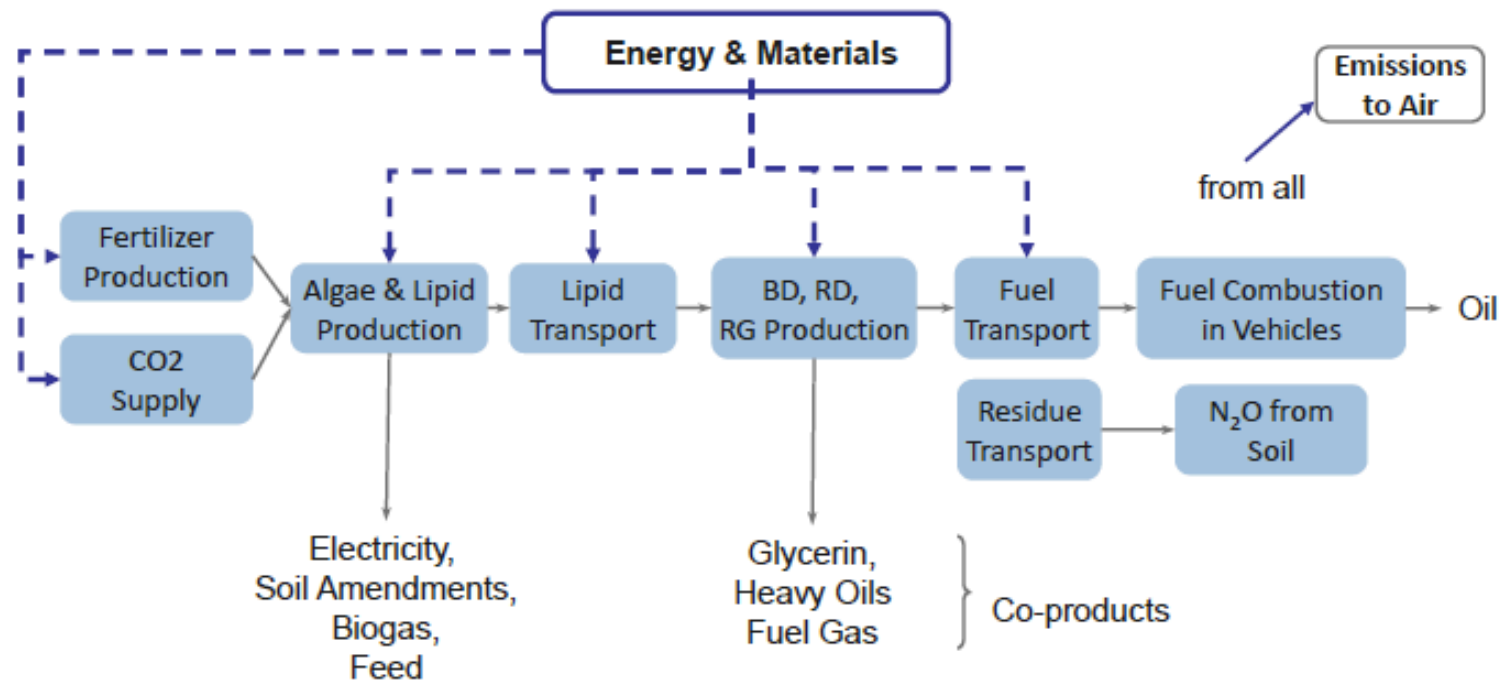

Figure 6 - APD Defined by GREET (Source: ANL, Dec 2011).

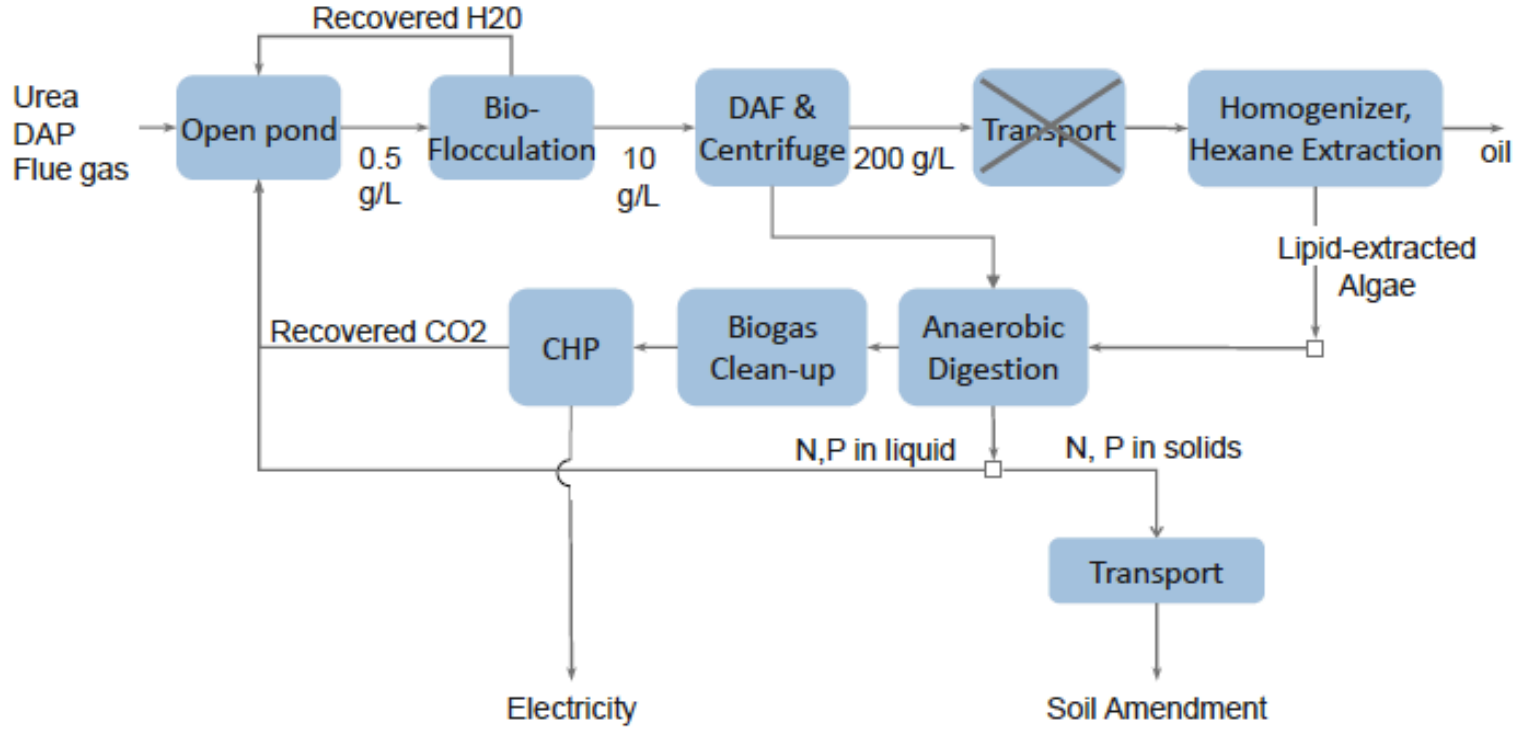

Figure 7 - Default Pre-Configured APD Defined by GREET (Source: ANL, Dec 2011). 
Once all process assumptions are plugged in, the emission balance calculated by APD is exported into the main GREET platform which models the emission balance for transport of the algal oil, and refining of the feedstock to AJF and it's distribution (ANL, 2012). The aviation module in GREET consists of detailed characterization of WTP, PTWa and WTWa pathways. The aviation module integrates jetfuel production processes from various feedstock sources into a single spreadsheet. For jetfuel derived from fossil fuels and biomass feedstock other than algae, the upstream processes - petroleum recovery and transportation for PJF, coal mining or natural gas recovery, processing for FT jetfuel or biologically derived oil production from plant or algal feedstocks - are in their original respective spreadsheets within GREET. The different aircraft classes, their operational characteristics, and the properties of conventional and alternative jetfuels are incorporated into the 'Jetfuel PTWa' sheet. The overall LCA results recording energy use and emissions results for various blends of PJF (with other jetfuel sources) are reported in the third 'Jetfuel WTWa' spreadsheet. Since 2011, ASTM approved HEFA jetfuels derived from vegetable oils in a 50:50 blend with PJF, a standard that is followed in this study (ANL, 2012).

Figure 8 shows the contribution of WTP and PTWa stages to $\mathrm{CO}_{2}$ equivalent emissions per MJ of alternative jetfuel sources including unconventional fossil fuel sources like oil sands and shale oil. Naturally the WTP stage for all bio-derived jetfuel pathways appears on the negative side of the GHG emissions scale owing to the atmospheric $\mathrm{CO}_{2}$ absorbed by photosynthesis during the biomass growth phase. However, the $\mathrm{CO}_{2}$ sequestered during biomass growth is negated by emissions released during biomass farming, collection, fertilizer application and hydrogen production needed for fuel upgrading. The carbon sequestered in the biomass is transferred to the fuel after the conversion step and returns to the atmosphere with the exhaust stream during fuel combustion in the aircraft engine (ANL, 2012). The AJF WTWa lifecycle emission balance is $25 \mathrm{gCO}_{2} \mathrm{e} / \mathrm{MJ}$ while crude oil derived PJF WTWa emission balance is significantly higher at $87.5 \mathrm{gCO}_{2} \mathrm{e} / \mathrm{MJ}$ (ANL, 2012) because of its dominating PTW emissions of $73.2 \mathrm{gCO}_{2} / \mathrm{MJ}$ (RAND, 2009).

It is important to note that in order to manage the complexity of a comprehensive LCA and limit it's scope and establish verifiable results, infrastructure-related activities are not included in the GREET boundary assumptions (ANL, 2012). 


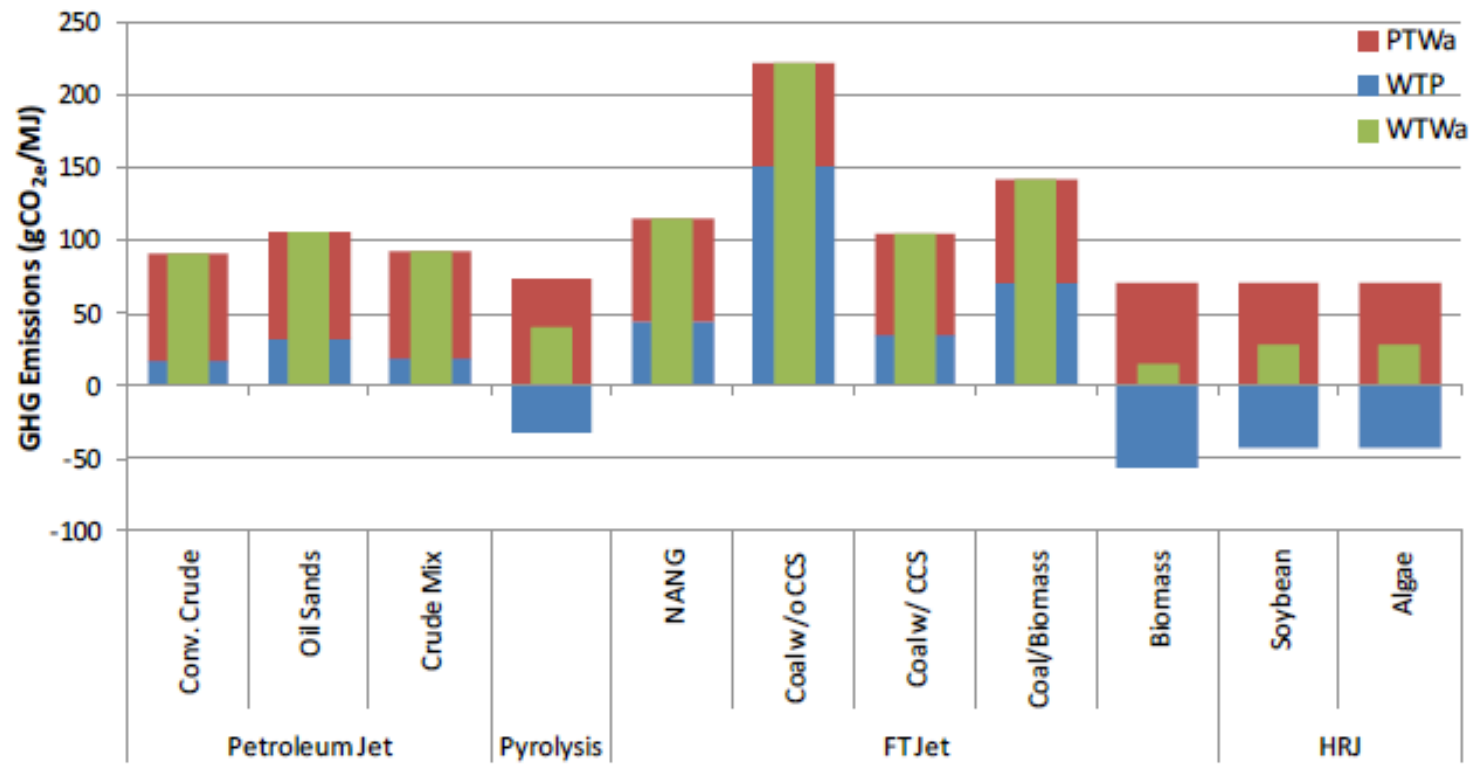

Figure 8 - GREET WTWa Evaluation for Alternative Jet Fuel Production Pathways (Source: ANL, 2012).

\subsubsection{Monetizing LCA Emission Using BC'S Carbon Tax}

Upon determining the appropriate LCA data, the final input for NPV analysis is derived from converting the GREET value into a revenue item. BC's carbon tax is employed to emulate a real life policy based carbon price. The carbon tax rate is currently at its highest rate of $\$ 30 / \mathrm{tCO}_{2} \mathrm{e}$. This is the emission penalty that the aviation industry will incur through consumption of jetfuel. As mentioned, because of the potentially lower lifecycle emissions posed by AJF in contrast to PJF, there is a real financial incentive for the aviation industry to embrace to AJF in order to save costs. The monetized algal emission benefit is the difference between PJF emission and AJF emission times the carbon tax.

\subsection{NPV CALCULATION}

The NPV is equal to the sum of all DCFs over ten years including the TV and UCC (Rosenbaum \& Pearl, 2009).

i) In other words NPV = Sum of DCFs (in ten years) + UCC + TV

NPV can be a positive, negative or neutral value depending on the economic potential or profitability of the investment venture. A positive NPV indicates that the investment assessed can be expected to provide a 
financial return that not only covers the liabilities (to lenders and equity holders) but provides a surplus; the magnitude of the positive NPV is proportional to the level of profitability. Similarly, a negative NPV indicates that any investment made in the project may not only be lost, but will likely incur further losses if any capital injected to keep the project running. Lastly, a neutral or zero NPV value indicates that the investment opportunity does not provide any positive or negative incentive for the investors to commit financially (Rosenbaum \& Pearl, 2009). Although the magnitude of the negative NPV indicates the extent to which the project will lose money, realistically investors are unlikely to financially commit to any opportunity that has a NPV equal or lower than zero. 


\section{DISCUSSSION}

\subsection{BASELINE ASSESSMENT}

The NPV analysis is undertaken by first determining net revenue generated per year, subtracting all costs from net revenues, and finally calculating the total DCF accounting for UCC and TV. There are two key assumptions associated with the hypothetical AJF production facility in question, namely that the facility reaches peak operational capacity (maximum oil production) by year 1 and, the facility generates the same algal oil output year after year i.e. the only difference in the net DCF generated year on year is caused by the DF. This simplified deterministic model is reflective of other valuation studies of hypothetical algal oil production facilities (Shiho et al, 2012). In reality production capacity both in the amount of algal oil produced and the refining capacity needed to accommodate the volume of algal oil produced is likely to be ramped up gradually within 1-3 years (Shiho et al, 2012). Additionally, the algal oil production capacity is likely to be subject to operational (maintenance, effect of weather, contamination etc.) and market perturbations (production level will follow demand) which will result in inconsistent yearly DCFs.

Under the baseline conditions (Table 1A) built on the current state of algal biofuel technology the NPV analysis results in a value of -2350.30 (Table 1B) implying that not only will investment made in the baseline AJF production value chain be lost, the investor is likely to lose a further $\$ 2,350.30$ million- the initial investment over the lifetime of the project. The primary reason for this is that under the baseline scenario i) the COP for AJF is high at $\$ 2.59 / 1$ compared to the much lower PJF COP at $\$ 0.84 /$ giving rise to negative revenue streams throughout the ten years, and ii) the very negative TV at - $\$ 940.57$ million and UCC of $\$ 947.30$ million. TV and UCC are each about $40 \%$ of the NPV value which signifies their dominance in the final NPV.

Due to the negative NPV value, under baseline technology state and policy condition, it can be safely deduced that the current AJF regime and low PJF price does not allow for profitable operation of AJF production. In order to investigate the influence of the key EBITDA elements, a sensitivity analysis is undertaken to determine the conditions under which AJF can be produced economically.

\section{Table 2A - Baseline Conditions}

\begin{tabular}{|l|l|}
\hline Parameter & Condition \\
\hline Algae oil content & $25 \%$ \\
\hline Pond productivity & $13.2 \mathrm{~g} / \mathrm{m}^{2} /$ day \\
\hline COP of PJF & $\$ 0.84 /$ liter \\
\hline COP of AJF & $\$ 2.59 /$ liter \\
\hline Carbon tax & $\$ 30 / \mathrm{tCO}_{2} \mathrm{e}$ \\
\hline
\end{tabular}




\section{Table 2B - Baseline NPV Assessment}

\begin{tabular}{|c|c|c|c|c|c|c|c|c|c|c|c|}
\hline \multirow[b]{2}{*}{ Source of Cash Flow (\$M) } & \multicolumn{11}{|c|}{ Year (N) } \\
\hline & 0 & 1 & 2 & 3 & 4 & 5 & 6 & 7 & 8 & 9 & 10 \\
\hline $\begin{array}{l}\text { Total Revenue from sale of } \\
\text { AJF }\end{array}$ & 0 & 33.02 & 33.02 & 33.02 & 33.02 & 33.02 & 33.02 & 33.02 & 33.02 & 33.02 & 33.02 \\
\hline $\begin{array}{l}\text { Total Revenue from } \\
\text { emissions benefit }\end{array}$ & 0 & 2.95 & 2.95 & 2.95 & 2.95 & 2.95 & 2.95 & 2.95 & 2.95 & 2.95 & 2.95 \\
\hline Total COP of AJF & 0 & 101.81 & 101.81 & 101.81 & 101.81 & 101.81 & 101.81 & 101.81 & 101.81 & 101.81 & 101.81 \\
\hline EBITDA & 0 & -65.84 & -65.84 & -65.84 & -65.84 & -65.84 & -65.84 & -65.84 & -65.84 & -65.84 & -65.84 \\
\hline Discount Factor & 1.00 & 1.07 & 1.14 & 1.23 & 1.31 & 1.40 & 1.50 & 1.61 & 1.72 & 1.84 & 1.97 \\
\hline Discounted EBITDA & 0.00 & -61.53 & -57.51 & -53.75 & -50.23 & -46.94 & -43.87 & -41.00 & -38.32 & -35.81 & -33.47 \\
\hline Terminal Value & - & - & - & - & - & - & - & - & - & - & -940.57 \\
\hline Upfront Capital Cost & -947.30 & & & & & & & & & & \\
\hline NPV & 2350.30 & & & & & & & & & & \\
\hline
\end{tabular}

\subsection{SENSITIVITY ANALYSIS}

\subsubsection{Impact of Changing JFP on NPV}

\section{Table 3A - JFP Conditions}

\begin{tabular}{|l|l|}
\hline Parameter & Condition \\
\hline Algae oil content & $25 \%$ \\
\hline Pond productivity & $13.2 \mathrm{~g} / \mathrm{m}^{2} /$ day \\
\hline COP of PJF & Variable \\
\hline COP of AJF & $\$ 2.59 /$ liter \\
\hline Carbon tax & $\$ 30 / \mathrm{tCO}_{2} \mathrm{e}$ \\
\hline
\end{tabular}

\section{Table 3B - JFP VS. NPV Assessment}

\begin{tabular}{|l|l|}
\hline Jetfuel Price (\$/1) & NPV \\
\hline 0.84 & -2350.30 \\
\hline 1.00 & -2216.27 \\
\hline 1.50 & -1797.33 \\
\hline 3.00 & -540.93 \\
\hline 6.00 & 2919.37 \\
\hline
\end{tabular}




\section{Graph 1 - Impact of Changing JFP on NPV}

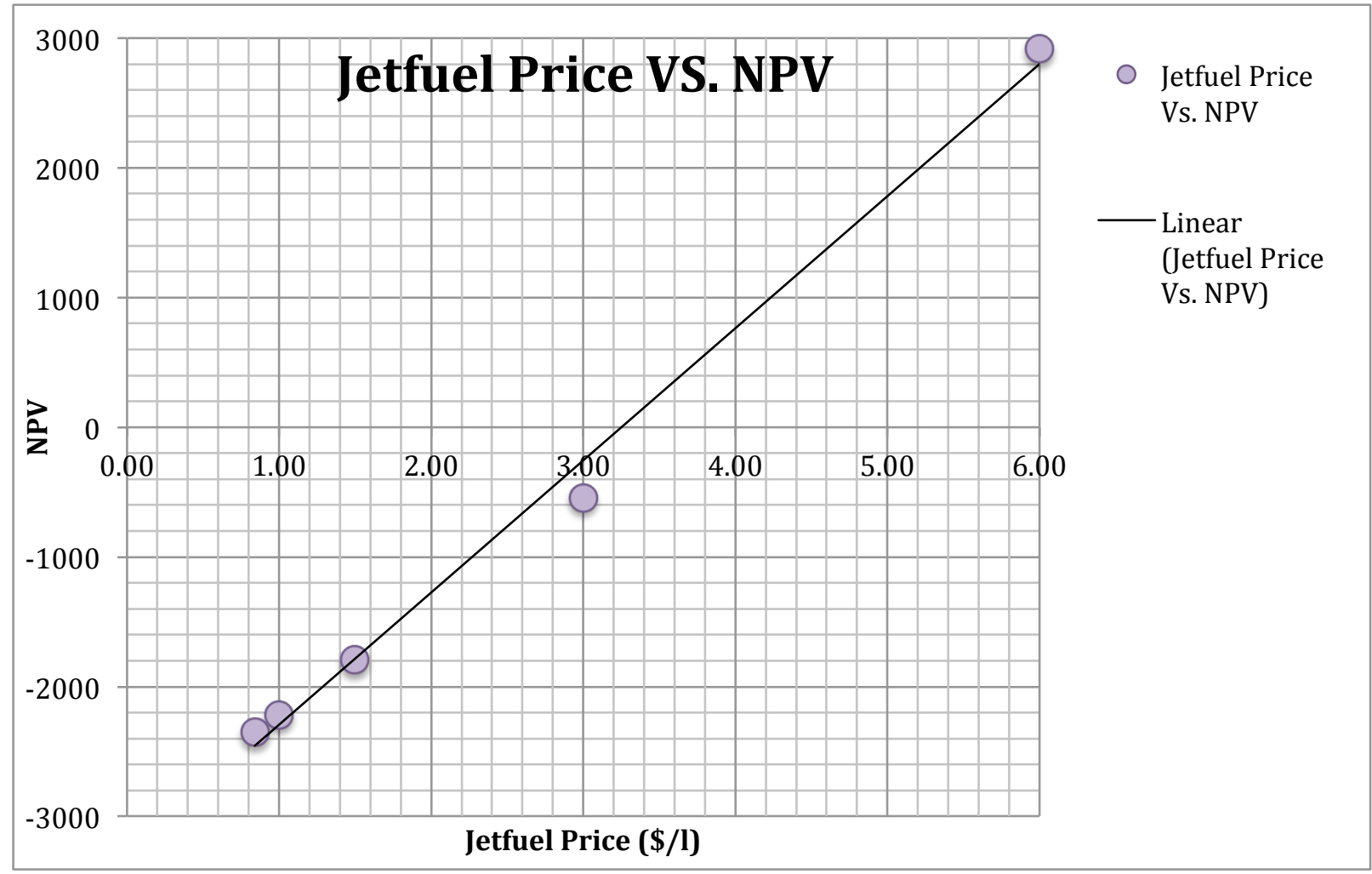

One of the primary motivations for the aviation industry to divert its dependency from PJF is due to high price volatility of PJF. PJF is sold at a price premium to crude oil prices. In 2012 the aviation industry paid about $\$ 200$ billion in fuel bills - almost 30\% of total operating costs paid by the industry every year (IATA, 2012). Sustainable non-fossil jetfuel produced from readily available biomass on the other hand offer the industry much needed reprieve in terms of supply and price security. As crude oil prices rise, biofuels become increasingly attractive as a tool to manage rising operating costs. Similarly it is widely accepted that rising oil prices also create a demand stimulus which translate into positive financial performance for alternative energy companies (Henriques \& Sadorsky, 2008). Oil prices have continually increased in recent years, quadrupling from 2000 to 2010 and will continue to rise posing a direct threat to JFP (Taylor et al, 2013).

The baseline scenario uses 2013 JFP to establish the profitability of AJF produced from a hypothetical algal oil facility. Given the augmenting effect of rising oil prices on the economic feasibility of competing RETs, it becomes a necessary exercise to forecast the profitability of such a facility as affected by changing JFP (Table 2B). Graph 1 illustrates the positive correlation between NPV and JFP as rising JFP directly augments the revenue streams from the sale of the product. The line curve intersects with the NPV axis at $\$ 3.20 / 1$ representing the Discounted Breakeven Price (DBP). This mirrors the argument posited by Galagher's 2011 study which highlighted the huge returns possible for algal biofuel producers if real crude oil prices were to rise significantly higher than $\$ 100$ per barrel or $\$ 0.84 /$ liter (Galagher, 2011). Under rising oil prices, the algal 
oil COP is increasing insensitive to loss of government subsidies and increases in CAPEX and/or OPEX as high profit margins from sale of AJF compensates for any other externally mediated CF perturbations (Galagher, 2011).

An important point to note is that a JFP increase from baseline to $\$ 1 / 1$ and $\$ 1.50 / 1$ shows a negligible impact on NPV, in contrast to doubling of JFP from $\$ 1.50 / 1$ to $\$ 3 / 1$ and onwards. But $\$ 3 / 1$ also translates to $\$ 381.44$ per barrel. Considering that IEA and the US EIA forecasts indicate a rising trend for oil prices with broad consensus on a future oil price in the range of US $\$ 100$ between 2015 and 2020, increasing to US $\$ 160$ per barrel by 2050 (CSIRO, 2011), \$381.44/l maybe be considered an unrealistic price instigator for the commercialization of AJF. Nevertheless, such extrapolation does offer insight into the expected performance of AJF technology and hints at producers to focus on non-market factors such as those inherent to the core algal oil technology to enhance the competitiveness of AJF in the near term.

\subsubsection{Impact of Carbon Tax on NPV}

\section{Table 4A - Carbon Tax Conditions}

\begin{tabular}{|l|l|}
\hline Parameter & Condition \\
\hline Algae oil content & $25 \%$ \\
\hline Pond productivity & $13.2 \mathrm{~g} / \mathrm{m}^{2} /$ day \\
\hline COP of PJF & $\$ 0.84 / \mathrm{liter}$ \\
\hline COP of AJF & $\$ 2.59 /$ liter \\
\hline Carbon tax & Variable \\
\hline
\end{tabular}

Table 4B - Carbon Tax VS. NPV Assessment

\begin{tabular}{|l|l|}
\hline Carbon Tax (\$/tCO2e) & NPV \\
\hline 30 & -2350.30 \\
\hline 60 & -2287.44 \\
\hline 120 & -2157.88 \\
\hline 200 & -1994.23 \\
\hline 400 & -1575.07 \\
\hline 800 & -736.98 \\
\hline 2000 & 1777.31 \\
\hline
\end{tabular}


Graph 2 - Impact of Changing Carbon Tax on NPV

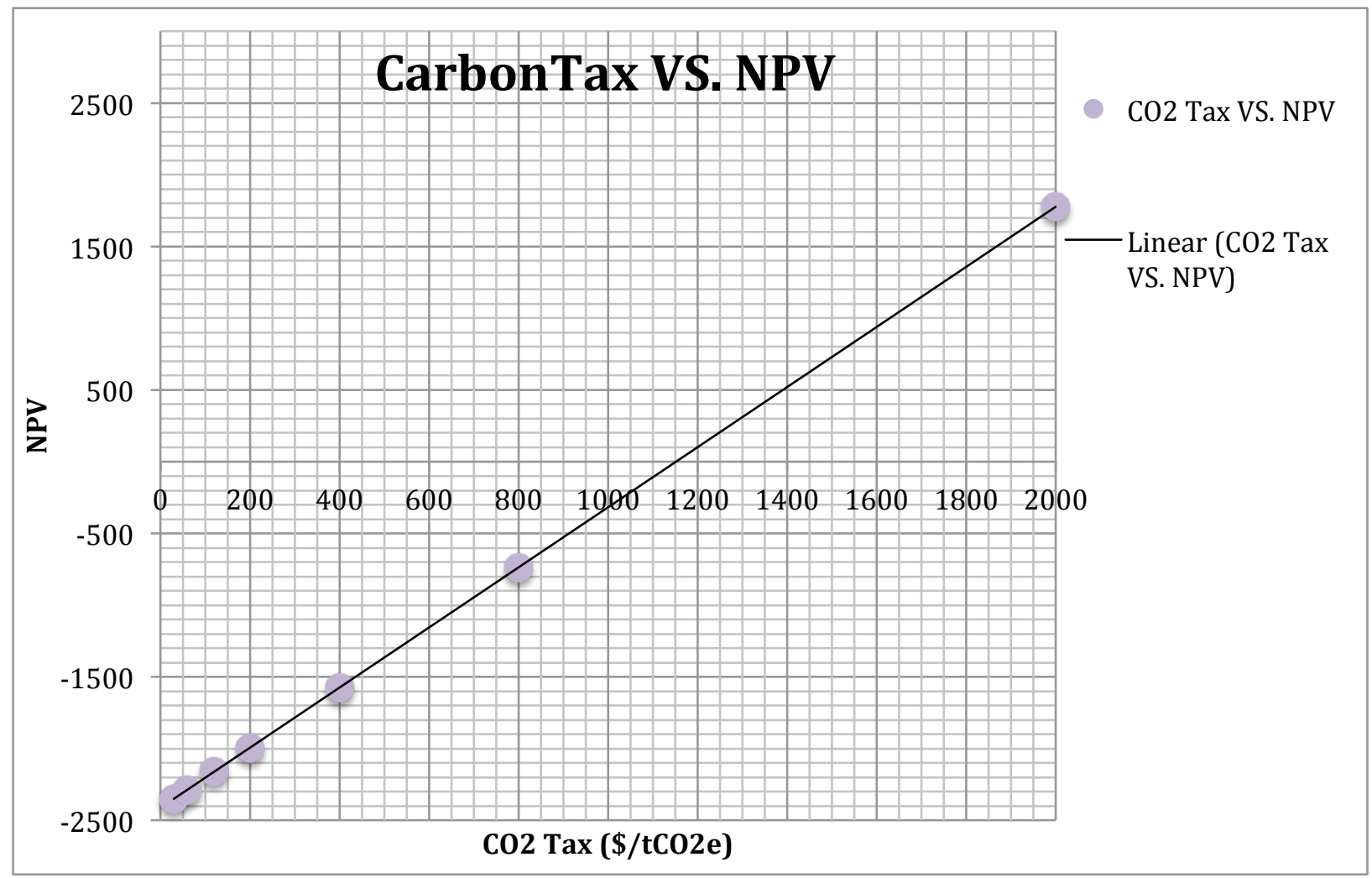

The carbon tax adds a premium to the cost of both AJF and PJF but because PJF imposes a significantly larger lifecycle $\mathrm{CO}_{2}$ footprint, airline operators using PJF will incur a larger emission penalty. AJF offers a lifecycle emission benefit of $62.5 \mathrm{gCO}_{2} \mathrm{e} / \mathrm{MJ}$ fuel consumed, which depending on the level of carbon tax applied, can offer producers and consumers a substantial financial reprieve. Therefore a rising carbon tax level is expected to make investment prospects for alternative energy technologies such as AJF more attractive as higher profitable returns can be expected from growing market adoption. This expectation is validated by the positive correlation between carbon tax levels and NPV shown in Graph 2. The initial rise of carbon tax levels from $\$ 30 / \mathrm{tCO}_{2} \mathrm{e}$ to $\$ 200 / \mathrm{tCO}_{2} \mathrm{e}$ has a minimal impact on NPV and in fact keeps NPV in the negative. In contrast, when the carbon tax doubles from $\$ 400 / \mathrm{tCO}_{2} \mathrm{e}$ to $\$ 800 / \mathrm{tCO}_{2} \mathrm{e}$, the NPV value also doubles approximately from -1575.07 to -736.98 (Table 3B). The DBP - where NPV is 0 - is about $\$ 1150 / \mathrm{tCO}_{2} \mathrm{e}$; when carbon tax is at any level above this point, revenues generated from AJF emissions benefit greatly compensates for the very high AJF COP.

It is important to note here that, as with the JFP scenario, extrapolation of carbon tax to extreme levels of $\$ 2000 / \mathrm{tCO}_{2} \mathrm{e}$ may not be practical because of the uncertainty with which the industry will respond to the heightened tax and how demand for aviation will shift. Higher carbon prices may lower the demand for air travel as airlines eventually pass on the extra costs to passengers; lower price scenarios such as the \$US 50/ 
$\mathrm{tCO}_{2} \mathrm{e}$ level set by the European Unions Emission Trading Scheme (EU ETS) have shown minimal impact on both demand and emissions in modeling scenarios. The industry is usually able to absorb higher carbon prices after the initial period of shock where profitability suffers from the implementation of the tax. As observed in 2008 when crude oil prices doubled, airlines tried to grapple with higher fuel prices by absorbing rising costs, leading to bankruptcies of almost twenty smaller airlines while the larger players announced reductions in their capacity and mergers. After a few years the industry redounded as airlines shed capacity and consolidated to be able to charge higher fares (Bonnefoy, 2011). Higher carbon taxes are expected to elicit similar responses. But even the models used to arrive at this conclusion only assume a carbon tax of $\$ 200 / \mathrm{tCO}_{2} \mathrm{e}$ to be the maximum tax level that can be practically applied without instigating the detrimental economic effects of making energy too expensive for economic activity (Bonnefoy, 2011). Similarly Timilsina et al concluded that on a global basis a carbon tax of $\$ 100-\$ 150$ in absence of other subsidies is the highest tax level that can push market penetration of biofuels without causing a significant loss in economic output (Timilsina et al, 2011).

The carbon tax versus NPV trend suggests that unless carbon tax is at an unrealistically high level, it will not impact the economic feasibility of the investment in any positive way, that is to say carbon tax alone, although can be a source of revenue, cannot make AJF profitable to produce. Therefore economically feasible production of AJF will once again have to rest on vast improvements in elements of the core technology and not on external policy conditions to push market adoption.

\subsubsection{Impact of Changing JFP and Carbon Tax on NPV}

Table 5A - JFP \& Carbon Tax Conditions

\begin{tabular}{|l|l|}
\hline Parameter & Condition \\
\hline Algae oil content & $25 \%$ \\
\hline Pond productivity & $13.2 \mathrm{~g} / \mathrm{m}^{2} /$ day \\
\hline COP of PJF & Variable \\
\hline COP of AJF & $\$ 2.59 /$ liter \\
\hline Carbon tax & Variable \\
\hline
\end{tabular}

Table 5B - JFP \& Carbon Tax VS. NPV Assessment

\begin{tabular}{|l|l|}
\hline Jet Fuel Price \$1/1 & \multicolumn{1}{|l}{} \\
\cline { 1 - 2 } Carbon Tax $\left.\mathbf{~} \mathbf{\text { NPV }} \mathbf{t C O}_{\mathbf{2}} \mathbf{e}\right)$ & -2216.27 \\
\hline 30 & -2153.41 \\
\hline 60 & -2023.85 \\
\hline 120 & -1860.19 \\
\hline 200 & \\
\hline
\end{tabular}




\begin{tabular}{|l|l|}
\hline 400 & -1441.04 \\
\hline 800 & -602.94 \\
\hline 2000 & 1911.34 \\
\hline
\end{tabular}

\begin{tabular}{|l|l|}
\hline Jet Fuel Price $\mathbf{\$ 1 . 5 0} / \mathbf{l}$ & \multicolumn{1}{|l}{} \\
\cline { 1 - 2 } Carbon Tax $\left.\mathbf{\$} / \mathbf{t C O}_{\mathbf{2}} \mathbf{e}\right)$ & -1797.33 \\
\hline 30 & -1734.47 \\
\hline 60 & -1604.90 \\
\hline 120 & -1441.25 \\
\hline 200 & -1022.10 \\
\hline 400 & -184.00 \\
\hline 800 & 2330.28 \\
\hline 2000 & \\
\hline
\end{tabular}

\begin{tabular}{|l|l|}
\hline Jet Fuel Price \$3/l & \multicolumn{1}{|l}{} \\
\cline { 1 - 2 } Carbon Tax $\mathbf{( \$ / \mathbf { t C O } _ { 2 } \mathbf { e } )}$ & $\mathbf{N P V}$ \\
\hline 30 & -540.93 \\
\hline 60 & -478.07 \\
\hline 120 & -348.51 \\
\hline 200 & -184.85 \\
\hline 400 & 234.30 \\
\hline 800 & 1072.40 \\
\hline 2000 & 3586.68 \\
\hline
\end{tabular}




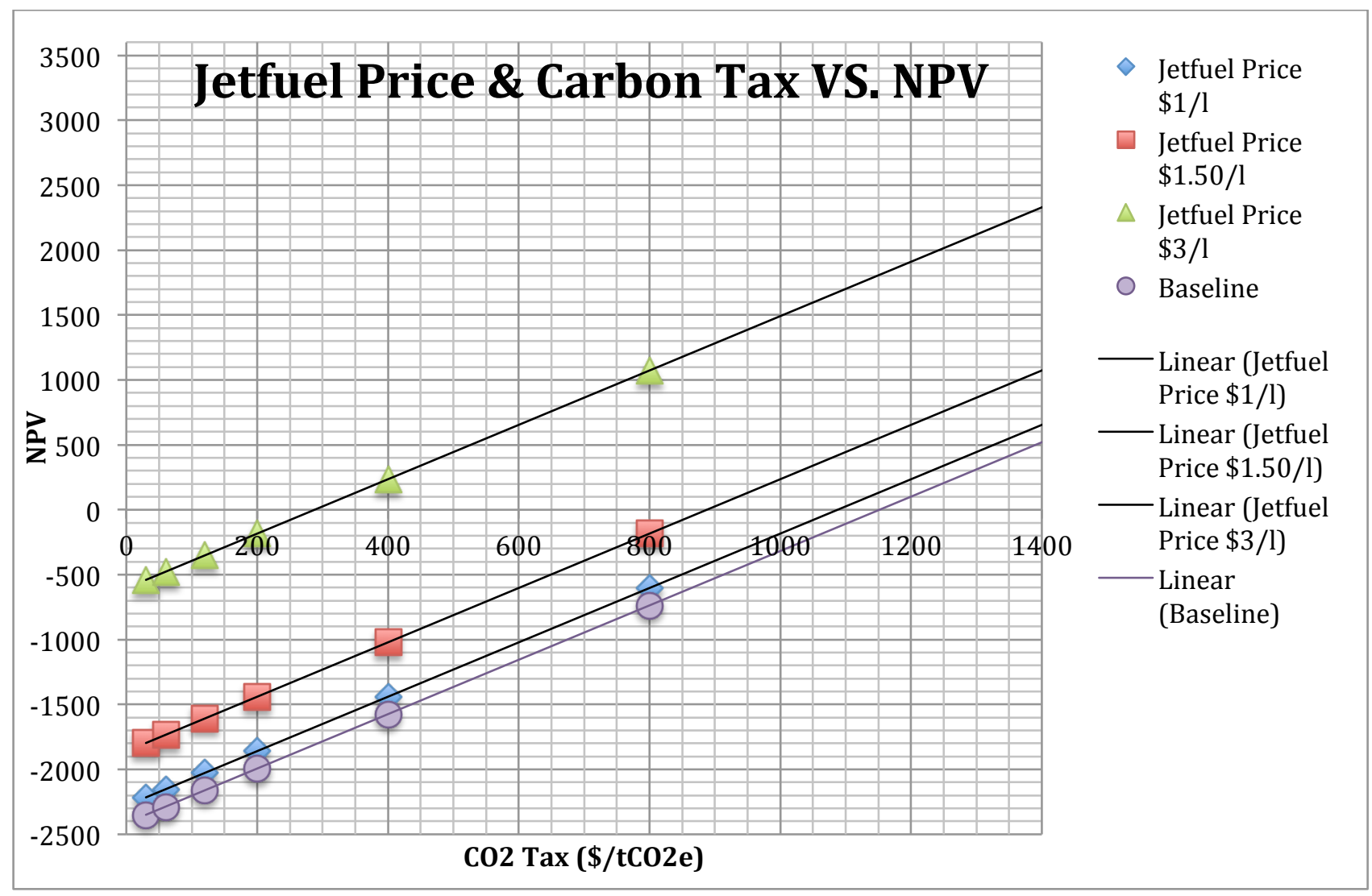

Individual trend lines for carbon tax levels and JFP versus NPV shown previously in Graph 1 and 2, indicate that these factors alone will not bring about a positive impact on the economic feasibility of AJF unless applied at unrealistically high levels. However since both carbon tax and JFP factor into the NPV calculation as revenue items, the combined effect of rising JFP variation in the presence of rising carbon tax levels can be investigated with the justification being that, both policy conditions and crude oil prices can shift simultaneously albeit not necessarily to the same degree.

The baseline assessment shows that JFP at $\$ 0.84 / \mathrm{l}$ and carbon tax at $\$ 30 / \mathrm{tCO}_{2} \mathrm{e}$, the DBP occurs at about $\$ 1160 / \mathrm{tCO}_{2}$ e. (Graph 3) However if JFP rises to $\$ 1 / \mathrm{l}$, the DBP shifts slightly to $\$ 1100 / \mathrm{tCO}_{2} \mathrm{e}$; at $\$ 1.50 / \mathrm{l}$ breakeven occurs at $\$ 900 / \mathrm{tCO}_{2} \mathrm{e}$; and finally when JFP is $\$ 3 / \mathrm{l}$ ( $\$ 476.28$ per barrel) the DBP falls to $\$ 300 / \mathrm{tCO}_{2} \mathrm{e}$. This overall trend of falling DBP is expected as rising JFP contributes a larger revenue stream into the NPV calculation and because the total amount of AJF production capacity is static year after year, the NPV depends less on the revenue contribution of AJF emission benefit. Effectively this means that under rising JFP, investors will rely less on external policy conditions to inspire investment in AJF production. 


\subsubsection{Impact of Changing Growth Scenario on NPV}

Table 6A - Growth Scenario Conditions

\begin{tabular}{|l|l|}
\hline Parameter & Condition \\
\hline Algae oil content & Variable \\
\hline Pond productivity & Variable \\
\hline COP of PJF & $\$ 0.84 /$ liter \\
\hline COP of AJF & $\$ 2.59 /$ liter \\
\hline Carbon tax & $\$ 30 / \mathrm{tCO}_{2} \mathrm{e}$ \\
\hline
\end{tabular}

Table 6B - Growth Scenario VS. NPV Assessment

\begin{tabular}{|l|l|}
\hline Growth Scenario & NPV \\
\hline 1. Low Growth/Baseline $\left(25 \%\right.$ oil $\& 13.2 \mathrm{~g} / \mathrm{m}^{2} /$ day) & -2350.30 \\
\hline 2. Medium Growth $\left(50 \%\right.$ oil $\& 40 \mathrm{~g} / \mathrm{m}^{2} /$ day) & -4259.40 \\
\hline 3. High Growth $\left(60 \%\right.$ oil $\& 60 \mathrm{~g} / \mathrm{m}^{2} /$ day) & -4568.81 \\
\hline
\end{tabular}

Graph 4 - Impact of Changing Growth Scenario on NPV




The yield of algal oil from a production facility is a function of two variables - the $\%$ by weight cellular content of oil (characteristic of the algal strain cultivated) and pond productivity which captures the mass of algal oil produced per unit area of pond per day. Since a higher yield of algal oil allows economies of scale by distributing the CAPEX and OPEX over large volume of end product ultimately pushing down COP, higher yield is an obvious objective for any algal oil production facility looking at a higher profit margin. However as discussed, the actual degree to which a particular production scheme impacts economies of scale is unclear in the absence of any fuel specific commercial algal cultivation. In 2008, an audit of twelve different sources of algal oil COP reflected a 50 -fold difference in the range of estimations reported. The disparity arose mainly from differences in algae growth assumptions, different cultivation systems, and baseline economic investment terms (Sun et al, 2011). Sustaining algal oil yield in an outdoor environment is very difficult and yet oil yield exhibits tremendous influence over LCA and TEA results particularly at baseline oil content and productivity levels; small changes at lower oil content and productivity levels lead to large shifts in price, energy consumption, and emissions (ANL et al, 2012).

The three growth scenarios picked for this part of the assessment represents both conventional performance as well as short and long-term strain improvements (selection of algal strains with higher oil content) and optimized pond configuration (providing higher productivity). The simulations were adopted from Davis et al (Davis et al, 2011), a seminal study that formed the foundation of the NREL harmonization exercise. The growth scenarios are positioned from low or baseline growth (data point $1-25 \%$ oil content and 13.2 $\mathrm{g} / \mathrm{m}^{2} /$ day productivity) simulated in most economic assessments of algal biofuels, medium growth (data point 2 - $50 \%$ oil content and $40 \mathrm{~g} / \mathrm{m}^{2} /$ day) scenario representing yield improvements consequent of optimization in the short term, and high growth scenario (data point $3-60 \%$ oil content and $60 \mathrm{~g} / \mathrm{m}^{2} /$ day) signifying the theoretical maximum achievable yield possible in the long term based on current technology trajectory (Table 5B). As evident from the Graph 4, there is a negative relationship between algal oil yield and NPV - the better the growth scenario the more negative the NPV. In this case, AJF is not economically feasible to produce regardless of the algal oil yield suggesting that, holding all other factors constant, no improvements in the biological and operational parameters will lead to profitable outcomes for the investment being contemplated. At first look this is counter intuitive as increasing algal yield should capture economies of scale, reduce COP and thus lead to more profitable production of AJF (Shiho et al, 2012). To a certain extent this is indeed the case looking at the growth scenarios concerned, the COP of AJF does go down from $\$ 2.59 / \mathrm{l}$, to $\$ 1.45 / \mathrm{l}$ and finally to $\$ 1.24 / \mathrm{l}$ in the medium and high growth scenarios respectively. However, because even the lowest AJF COP is still higher than baseline JFP of $\$ 0.84 / 1$, AJF production under the present scenario conditions will incur loses regardless of the algal oil yield, in fact the losses will stack up higher at higher yields because more biomass will be sold at a loss.

Algal biomass cultivation is inherently different from cultivation of $1^{\text {st }}$ generation feedstock. Algal oil COP does not appear to exhibit a strong correlation with production volume due to the dilute nature of the algal 
suspension. This makes it difficult to capture economies of scale simply by ramping up cultivation capacity. As cultivation volume increases so does the need for capital investment in adjacent processing capacity inadvertently preventing dilution of CAPEX and impeding economies of scale (Sun et al, 2011). The total system throughput for a 39.31 MLY algal oil production facility, due to the high dilution factor, is significantly higher than 39.31 MLY of raw algal suspension; the baseline facility flow rate is higher than the largest wastewater treatment plants in the US. Therefore, pursuing economies of scale through volume capacity exclusively may only serve a limited advantage in reducing COP at commercial scale operations (this could change as underlying processing sub-technologies are tailored specifically to algal fuel derivatives (Davis et al, 2011).

Additionally, algal oil content also impacts COP more strongly than productivity does. In open ponds, when oil content is either doubled or halved, the associated net cost impact is twice as that elicited by a similar adjustment in productivity. This is because increasing the productivity merely shrinks the size of the growth system required relative to the amount of algal biomass being grown, whereas an increase in oil content actually altogether decreases the amount of algal biomass throughput that must be processed to attain oil production targets, thereby instigating reduction in all downstream processing costs (Davis et al, 2011).

Finally, the cost improvement from baseline to medium growth scenario is substantially larger than the cost improvement from the medium to high growth scenario. This reflects likely realistic outcomes as medium growth parameters are practically achievable, while the high growth parameters by virtue of being the theoretical maximum will be much more difficult to reach (Sun et al, 2011). It is important to note that the high growth scenario in this case merely represents the maximum oil content and productivity achievable within the context of the baseline configuration and it's associated assumptions. Thus, while the contingent COPs may hint at future cost ranges, it does not mean that they are the absolute lowest COPs attainable. It is possible that numerous yet unforeseen process options and technological advancements in the future will push down COP further (Davis et al, 2011).

\subsubsection{Impact of Changing Growth Scenario and JFP on NPV}

Table 7A - Growth Scenario \& JFP Conditions

\begin{tabular}{|l|l|}
\hline Parameter & Condition \\
\hline Algae oil content & Variable \\
\hline Pond productivity & Variable \\
\hline COP of PJF & Variable \\
\hline COP of AJF & $\$ 2.59 /$ liter \\
\hline Carbon tax & $\$ 30 / \mathrm{tCO}_{2} \mathrm{e}$ \\
\hline
\end{tabular}


Table 7B - Growth Scenario \& JFP VS. NPV Assessment

\begin{tabular}{|l|l|}
\hline \multicolumn{2}{|l|}{ Low Growth (Baseline Scenario) (25\% oil \& 13.2 g/m2/day) } \\
\hline Jetfuel Price (\$/l) & NPV \\
\hline 0.84 & -2350.30 \\
\hline 1.00 & -2216.27 \\
\hline 1.50 & -1797.33 \\
\hline 3.00 & -540.93 \\
\hline 6.00 & 2919.37 \\
\hline
\end{tabular}

\begin{tabular}{|l|l|}
\hline \multicolumn{2}{|l|}{ Medium Growth $(50 \%$ oil \& $40 \mathrm{~g} / \mathrm{m} 2 /$ day) } \\
\hline Jetfuel Price (\$/1) & NPV \\
\hline 0.84 & -4259.40 \\
\hline 1.00 & -2718.83 \\
\hline 1.50 & 376.98 \\
\hline 3.00 & 9663.79 \\
\hline 6.00 & - \\
\hline
\end{tabular}

\begin{tabular}{|l|l|}
\hline \multicolumn{2}{|l|}{ High Growth $(60 \%$ oil \& $60 \mathrm{~g} / \mathrm{m} 2 /$ day $)$} \\
\hline Jetfuel Price (\$/1) & NPV \\
\hline 0.84 & -4568.81 \\
\hline 1.00 & -2785.65 \\
\hline 1.50 & 2786.51 \\
\hline 3.00 & 19502.81 \\
\hline 6.00 & - \\
\hline
\end{tabular}




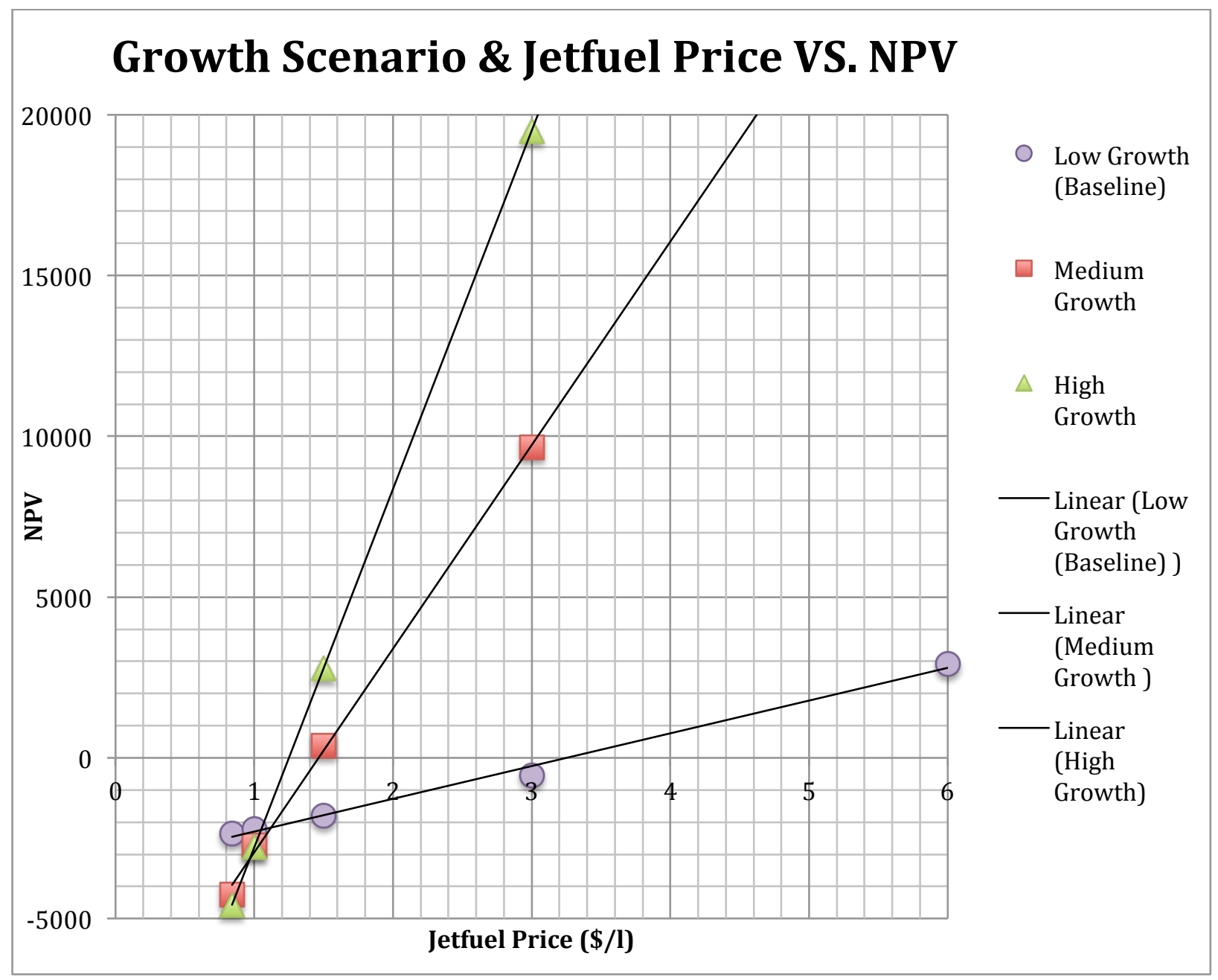

Attempting to commercialize AJF under the baseline JFP is futile as even the lowest possible COP leads to a very negative NPV. This brings to question how the commercialization scenario for AJF changes under increasing JFP, a trend that is inevitable as upward shifts in crude oil prices are prompted by shrinking supply. However when enhanced growth scenarios are assessed under shifting JFP as in Graph 5, all growth scenarios now present at least some positive return-on-investment potential. The trend lines grow increasingly steeper as oil content and productivity rise; for any JFP, the corresponding NPV is highest for high growth scenario, followed by medium growth and baseline growth scenario. The high growth scenario, presenting the theoretical maximum algal oil yield, captures large economies of scale and becomes profitable at any JFP above the DBP of $\$ 1.30 /$ liter. Similarly, the medium growth scenario becomes profitable at any JFP above $\$ 1.40 /$ liter and low growth scenario above $\$ 3 /$ liter; the difference in DBP between medium and low growth scenario is significant because COP falls from $\$ 2.59 /$ liter to $\$ 1.45 /$ liter - a cost improvement of $44 \%$ triggered by a corresponding jump in oil content and productivity from $25 \%$ to $50 \%$ and $13.2 \mathrm{~g} / \mathrm{m}^{2} /$ day to 40 
$\mathrm{g} / \mathrm{m}^{2} /$ day respectively. This margin is much lower for the jump between medium and high growth scenario due to the lower boost in yield performance, jumping from $50 \%$ oil content and $40 \mathrm{~g} / \mathrm{m}^{2}$ day, to $60 \%$ oil content and $60 \mathrm{~g} / \mathrm{m}^{2}$ day which corresponds to a cost reduction from $\$ 1.45 /$ liter to $\$ 0.90 /$ liter (cost improvement of 38\%).

\subsubsection{Impact of Changing Growth Scenario and Carbon Tax on NPV}

\section{Table 8A - Growth Scenario \& Carbon Tax Conditions}

\begin{tabular}{|l|l|}
\hline Parameter & Condition \\
\hline Algae oil content & Variable \\
\hline Pond productivity & Variable \\
\hline COP of PJF & $\$ 0.84 /$ liter \\
\hline COP of AJF & $\$ 2.59 /$ liter \\
\hline Carbon tax & Variable \\
\hline
\end{tabular}

Table 8B - Growth Scenario \& Carbon Tax VS. NPV Assessment

\begin{tabular}{|c|c|}
\hline \multicolumn{2}{|c|}{ Low Growth (Baseline Scenario) (25\% oil \& $13.2 \mathrm{~g} / \mathrm{m} 2 /$ day } \\
\hline Carbon Tax $\left(\$ / \mathrm{tCO}_{2} \mathrm{e}\right)$ & NPV \\
\hline 30 & -2350.30 \\
\hline 60 & -2287.44 \\
\hline 120 & -2157.88 \\
\hline 200 & -1994.23 \\
\hline 400 & -1575.07 \\
\hline 800 & -736.98 \\
\hline 2000 & 1777.31 \\
\hline
\end{tabular}

\begin{tabular}{|l|l|}
\hline \multicolumn{2}{|l|}{ Medium Growth (50\% oil \& 40 g/m2/day) } \\
\hline Carbon Tax (\$/tCO2e) & NPV \\
\hline 30 & -4259.40 \\
\hline 60 & -3794.86 \\
\hline 120 & -2865.56 \\
\hline 200 & -1626.85 \\
\hline 400 & 1470.45 \\
\hline 800 & 7664.00 \\
\hline 2000 & - \\
\hline
\end{tabular}




\begin{tabular}{|l|l|}
\hline \multicolumn{2}{|l|}{ High Growth $(60 \%$ oil \& $60 \mathrm{~g} / \mathrm{m} 2 /$ day $)$} \\
\hline Carbon Tax (\$/tCO2e) & NPV \\
\hline 30 & -4568.81 \\
\hline 60 & -3732.42 \\
\hline 120 & -2060.07 \\
\hline 200 & 169.95 \\
\hline 400 & 5744.88 \\
\hline 800 & 24567.96 \\
\hline 2000 & 50340.13 \\
\hline
\end{tabular}

Graph 6 - Impact of Changing Growth Scenario and Carbon Tax on NPV

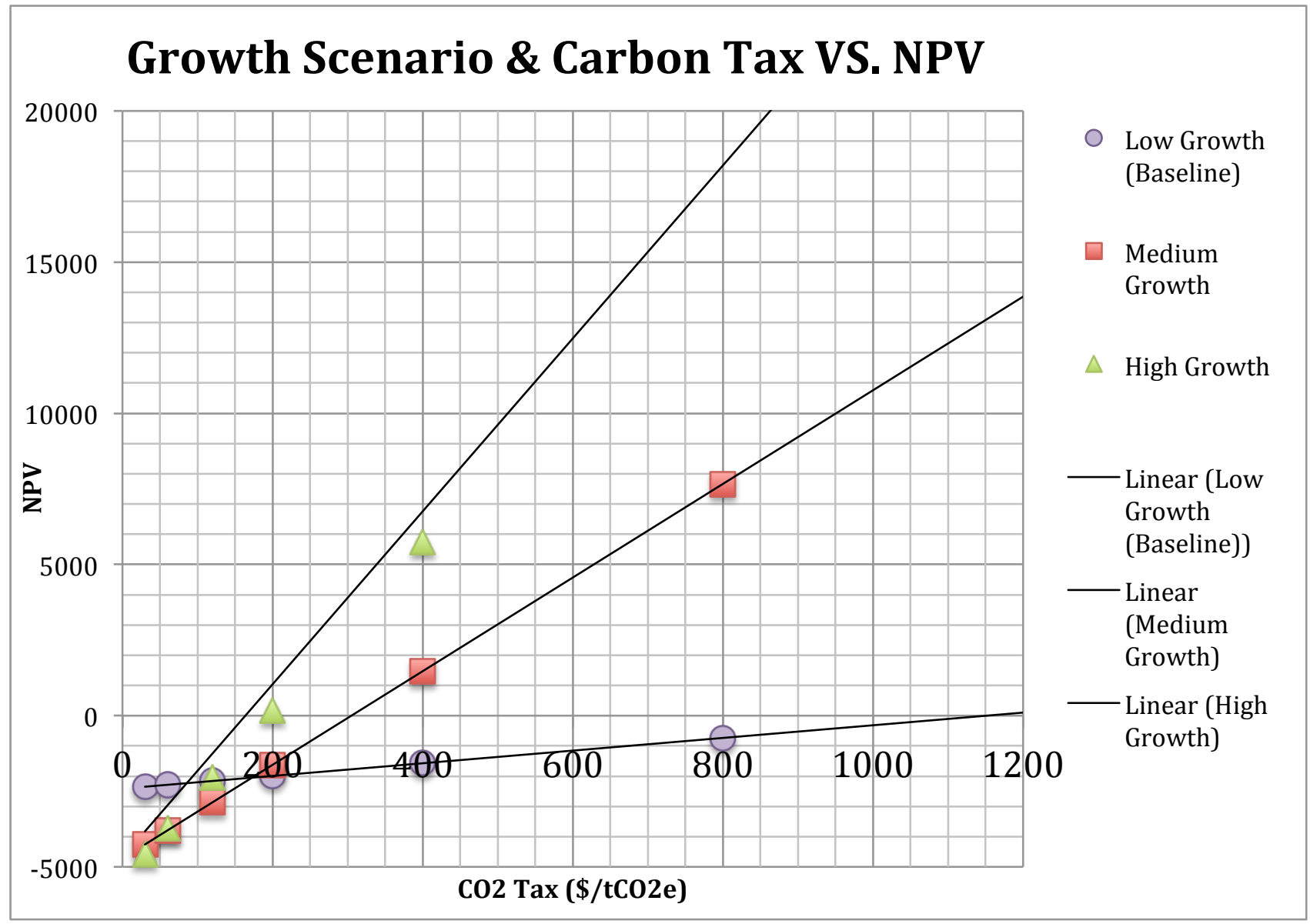

The revenue generated from carbon tax mediated emission benefit is small and therefore exudes minor influence on the overall NPV relative to the primary revenue stream generated from the sale of AJF. As evident from Graph 2, carbon tax has minimal impact on NPV unless the tax rate is unrealistically high. However as the algal biofuel technology develops manifesting in higher yield, it is worthwhile to know how a changing carbon tax regime can impact the commercialization potential of AJF. 
Graph 6 illustrates the relationship between growth scenario and carbon tax levels versus NPV levels, which resemble the relationship, illustrated in Graph 5. This is because at low algal oil yield, the monetized emission benefit as a proportion of total revenue, is larger than the proportion under equivalent carbon tax levels at higher growth scenarios i.e. for the same carbon tax level, more revenue comes from the sale of AJF at higher yields than revenue from emissions benefit. For example, below $\$ 140 / \mathrm{tCO}_{2} \mathrm{e}, \mathrm{NPV}$ for the low growth scenario is higher for every carbon tax level than corresponding NPVs under medium and high growth scenarios.

Above $\$ 200 / \mathrm{tCO}_{2} \mathrm{e}$, the carbon tax is high enough making the emissions benefit sufficient to garner a growing proportion of the net revenue, eventually generating a high net positive revenue stream for a positive NPV (at least under high growth scenario).

The greater the volume of AJF produced, the greater the emissions avoided through the displacement of PJF. Following from this it is obvious that at low algal oil yield, carbon tax will be required at much higher levels than at medium and high growth scenarios to elicit a positive NPV. This is indeed the case. The low growth scenario has a DBP of $\$ 1150 / \mathrm{tCO}_{2} \mathrm{e}$ compared to $\$ 300 / \mathrm{tCO}_{2} \mathrm{e}$ and $\$ 200 / \mathrm{tCO}_{2} \mathrm{e}$ under medium and high growth scenarios respectively indicating that, when all other factors are constant, a carbon tax regime can perhaps enhance the commercialization potential of AJF only if drastic technology advancements can push oil content and productivity levels to the medium growth scenario, a possibility that is attainable in the short term through heavy investment and focus on R\&D. 


\section{RECOMMENDATIONS}

The world is facing converging challenges in maintaining energy security and managing the threat of runaway GHG emissions. Biofuels offer promise as a response to both of these critical issues. Biofuel research activity peaked during the oil crises of the 1970s and has until the last decade or so fluctuated with the oil price. Recent crude oil price volatility and heightened awareness of climate change related impacts has ushered a renaissance of interest in sustainable fuels. Traditional food-based biofuels have seen incredible growth in the last decade pushed largely by sustainability and energy security policy goals. But developed in a narrow window of time and opportunity, the propagation for $1^{\text {st }}$ generation biofuels have come at substantial environmental and social costs. Yet the strong imperative to deliver effective energy solutions still exist and call for integrated R\&D and policy formulations across all the diverse sectors constituting a biofuel value chain is more urgent than ever before ( $0^{\prime}$ Connell \& Haritos, 2010). The aviation industry is at the forefront of this call, recognizing that advanced non-food based biofuels provide a means to generate low resource intensive and regionally produced jetfuel that can stabilize price volatility and reach emissions targets, both of which are vital for the industry's continuing growth in the coming decades.

AJF has the potential to meet all or a significant portion of the fuel needs in the sector by virtue of high algal productivity, substantially lower demand for agricultural inputs compared to $1^{\text {st }}$ generation biofuel pathways, and an ability to assimilate multiple waste streams as part of the algal growth cycle. But in order to offer real reprieve to aviation, AJF has to be produced at a large enough scale, offer substantial emissions benefit (at the minimum to make AJF carbon neutral), and at a price that is competitive with PJF. AJF is still far from achieving commercial competency in any of these areas.

As demonstrated in this study, an investment made today in a hypothetical AJF value chain, under baseline or current state of technology, will not yield a profitable outcome which is the only outcome that can valorize the investment decision. This study investigates the impact of factors inherent to a conventional algal oil production schematic and the influence of external factors like JFP and carbon tax on the profitability of AJF production. JFP and carbon tax in isolation can bring about the desired positive NPV albeit to various degrees, but only at levels that are unrealistically high. In the case of enhancing algal oil yield in isolation, even the theoretical maximum algal yield cannot generate an AJF COP lower than the current baseline JFP. This indicates that any improvement in the core technology parameters can be justified only under a JFP that is higher than the lowest theoretical AJF COP possible; a carbon tax will contribute marginal revenue thereby making the overall investment scenario more attractive. The sensitivity analysis further reveals the affect of interacting factors on the overall economic feasibility and commercialization potential of AJF. Particularly interesting here is the combined impact of elevating carbon tax levels and enhanced growth scenarios versus NPV, as well as elevating JFP and enhanced growth scenarios versus NPV. These two combinatorial forecasts provide valuable insights into the technological (including the technoeconomic and lifecycle performance of 
AJF production) and policy context requisite to the profitable operation of an AJF value chain. The following sections elaborate on the most effective strategies, derivative of the NPV analysis, aiming to highlight opportunities and ultimately guide R\&D scientists, technology developers, industry stakeholders and policy makers. The following section outlines two sets of recommendations in technological, and policy.

\subsection{CORE TECHNOLOGY}

\subsubsection{Raise CAPEX Efficiency}

Algae oil production systems are a complex composite of sub- technologies in production, harvesting, extraction, drying systems etc. Optimal integration and utilization of process steps is essential to operating easier, better, and lower cost systems (Singh \& Gu, 2010). One of the most efficient applications of capital in the production pathway comes from 'squeezing' as much product out of a system as possible to achieve economies of scale. Because feedstock price can often account for up to $40 \%$ of final COP of advanced biofuels, and (in the context of this study) the excess refinery capacity downstream (300 MLY capacity accommodating only 39.31 MLY algal oil), the prime target to apply CAPEX and OPEX efficiency measures is at the algal oil production process. However, unlike $1^{\text {st }}$ generation biofuels, the production capacity in algae farms cannot be ramped up simply by cultivating more algae per unit of pond (Singh \& Gu, 2010). The profitability of small farms is naturally quite low due to the high UCC relative to the low oil yield; profitability can rise rapidly with increased size but soon stabilizes suggesting the poor scalability of currently implemented harvest and process technologies (Stephens et al, 2010) - a case particularly true of farms smaller than 200 ha (Singh \& Gu, 2010). This is because algae grow at highly dilute concentrations and simply growing more algae requires an equivalent ramp up in processing capacity which inadvertently prevents the 'dilution' of CAPEX.

The primary instigator of AJF commercialization will come from cultivating 'fatter' and 'faster' algal strains. Advanced genetic manipulation techniques that enable selective 'fine-tuning' of the DNA of potential high-yield strains can optimize the delivery of desired oil types (Schenk et al 2008). Algae biofuel producers have to identify high-oil algal species that can generate bio-crude for drop-in fuels in the quantities and price points needed. Most algal strains used today have only $25 \%$ oil content (used in baseline scenario) but targeted R\&D can facilitate leaps in oil yield in the short term, engineering common algal strains to constitute $50 \%$ oil (medium growth scenario), and eventually even higher composition at the theoretical maximum level of $60 \%$ (high growth scenario) in the long term (Davis et al, 2011). Algal oil producers using fatter algae with $60 \%$ oil content can benefit substantially from the significant CAPEX and OPEX reductions compared to using species with baseline performance (Singh \& 
Gu, 2010). Advances in biotechnology can also simplify or cut short process steps all together. For example, Origin Oil combines harvest and extraction steps by engineering algal cells as mini-refineries in a process referred to as 'milking the algae' whereby the algae excretes hydrocarbon fuels directly into the growth medium (Singh \& Gu, 2010). Arizona State University took this approach a step further by creating a blue-green algae that directly excretes a type of kerosene jetfuel into the growth medium thereby bypassing the harvest, extraction and refining systems all together (Singh \& Gu, 2010). Additionally, due to the differences in the biochemical pathways in multi-cellular terrestrial plants and unicellular algae with respect to quality and quantity of the oil synthesized during nutrient deprivation (Packer 2009), a database mapping the chemical composition of oils associated with various algal strains along with the requisite process conditions (such as factors influencing the interaction and extraction of oil using solvents) can become a critical asset to algal biotechnologists (Greenwell 2011).

Enhanced economic performance can also be attained from better process design and modeling tailored specifically to algal cultivation for fuel derivatives. Current near-term cost estimates for harvest and extraction technologies are borrowed largely from technologies applied in the wastewater treatment. Newer harvesting technologies like electrocoagulation adopted from agricultural practices used instead of conventional flocculants, can provide a $50 \%$ capital cost reduction compared to the baseline configuration. But by and large, novel process technologies are yet to be developed and implemented. In their absence, it is useful to understand the cost-cutting potential of generic measures to identify realistic improvements that can translate to overall economic benefits for algal oil production in the short-term (ANL et al, 2012).

Like most RETs, algal oil COP is largely driven by CAPEX rather than OPEX (Sun et al, 2011), a fact that is particularly true at the low baseline productivity of $13.2 \mathrm{~g} / \mathrm{m}^{2} /$ day (ANL et al, 2012). As Figure 9 shows, amongst the various CAPEX contributors, the pond liner is substantial, comprising up to $60 \%$ of the total installed CAPEX while providing very limited utilization at baseline performance. When productivity increases the relative cost contributions of pond liners and fixed operating cost will dilute relative to other cost allocations. But this requires optimal algal strain development (ANL et al, 2012). Until R\&D advancements allows for this, pond liner cost can be reduced through fundamental redesign of pond systems or relaxing land grading and excavation requirements for building algal farms; in combination these two activities can potentially reduce CAPEX by up to 30\%. Alternatively liners can be removed altogether. Liners are traditionally used to reduce leakage (both water consumption and contamination issue) and manage high circulation costs characteristic of unlined ponds. However liners are not a technical requirement for pond operability if the host soil characteristics are satisfactory (for example in term of adequate soil compaction, clay composition and infiltration of worm holes); removing liners may limit algal farms to certain topographical profiles, but the option may still be worth pursuing for candidate areas given that removing liners can cut CAPEX by 45-55\% (ANL et al, 2012). 
Although it is beyond the immediate reach of this study, it is worth noting that there is a $2^{\text {nd }}$ popular, albeit even more nascent, cultivation technology currently being explored in laboratory and pilot settings. The modular technology called Photobioreactors (PBRs) comprises a light permeable artificially illuminated algal culture reactor, a gas exchange system that feeds $\mathrm{CO}_{2}$ and removes photosynthetic $\mathrm{O}_{2}$ (toxic to algae), and a harvesting system that concentrates the algae for downstream processing (Knoshaug 2011). In contrast to open ponds, PBRs, being closed systems, negate issues of evaporation and contamination by broader species of algae, as well as allowing integration of real-time control over ambient conditions and biological inputs (Knoshaug 2011) that ultimately manifests in substantially larger biomass yields per unit volume of water than open pond cultivation.

Having said that, currently the very high PBR material and energy input costs (to cool the naturally illuminated systems and moderate culture condition in real time) are a major impediment to taking advantage of the CAPEX efficiency benefits that could otherwise be experienced from the easily scalable modular technology (Knoshaug 2011). But as R\&D continues, as it is in open pond cultivation, efficient reactor designs (that can maximize light penetration and mixing by sheltering algal cells from photodegradation brought about by over exposure) will facilitate higher photosynthetic activity (Schenk et al 2008) and consequently larger productivity. Additionally, hybrid systems incorporating characteristics of both open and closed systems are also being investigated. Under such schemes, algae "are grown continuously in PBRs under nutrient sufficient conditions and then a portion is transferred to nutrient limited open ponds to induce oil production. Enough nutrients are transferred with the inoculum for the culture to grow for 1 day, and after 3 days when lipid production has peaked, open ponds are harvested, cleaned and re-inoculated" (Schenk et al 2008, pg. 22).

\subsubsection{Raise OPEX Efficiency}

OPEX factors play a relatively minor role in influencing the economics of AJF production in contrast to the dominating role of CAPEX factors in establishing the final COP. The NREL harmonization study notes the low sensitivity of a handful of operational parameters that marginally impact total OPEX (but not the overall project cost), highlighting opportunities to further lower fixed operating costs which constitute roughly $60 \%$ of the baseline OPEX (ANL et al, 2012). As Figure 9 illustrates, the main non-technology determinant of operational efficiency is the number of operational days per year the algal oil production facility is expected to be functional. In the baseline scenario, the facility operates 330 days/year after accommodating pond crashes and other maintenance 'down time'. If the factor is decreased to 250 days/year - as can occur due to pond freezes, increased pond crashed and re-inoculation, etc. - the loss in production yield raises price substantially i.e. when algal oil is used as feedstock for biodiesel instead of jetfuel, a 250 days/year operational schedule adds up to $\$ 1.40 / 1$ to the biodiesel COP. This reiterates the importance of a robust algal 
strain, building the facility where freezing and culture contamination is minimal (ANL et al, 2012).

Nutrient cycle is another crucial operational factor. From a cost standpoint, an AD utilizes spent biomass to recycle nutrient in addition to generating methane gas for onsite power generation via a Combined Heat-andPower process. The nutrient cost is considerable but not as highly impactful on the overall OPEX as evident by its mediocre cost sensitivity to change in nutrient recycle rates (ANL et al, 2012).

It is worth noting here that despite freshwater being the baseline growth medium, OPEX shows low sensitivity to water consumption as varying evaporative water losses in orders of magnitude corresponds to only trivial cost impacts. Furthermore the cost impact observed the consequence of merely adding a water blowdown stream - to manage increasing salinity in growth ponds due to evaporation - which leads to loss of algal biomass that could otherwise be processed downstream.

While assumptions on water recycling and evaporation rate do not appear to show a notable cost sensitivity, it is important to iterate the underlying assumption that freshwater does incur a cost beyond the capital and power expenses for pumping it from underground reservoirs. However in the event that the regional water demand rose or algal farms have to purchase water from a utility, the cost impact would be noticeably higher - by as much as $\$ 0.26 / \mathrm{l}$ of freshwater according to one estimate (Davis et al, 2011). The baseline scenario assumes the algal oil facility is built in a geographical location with enough groundwater resources to supply both the domestic demands of the region and the algal farm while preserving the reservoir though natural replenishment. Of course the use of freshwater does bring to light the obvious sustainability concerns of industrial scale consumption and the entailing restriction placed on potential algal cultivation farms by regional variability in consumption patterns, weather and water availability (ANL et al, 2012). Even if large variations in the water balance parameters show low-cost sensitivity, the impact of industrial extraction on local water resources will be much more dramatic and raise the issue of sustainability (Davis et al, 2011). The NREL concedes that future studies will need to investigate the feasibility of algal cultivation using seawater or brackish water to limit the sustainability impact (ANL et al, 2012). 


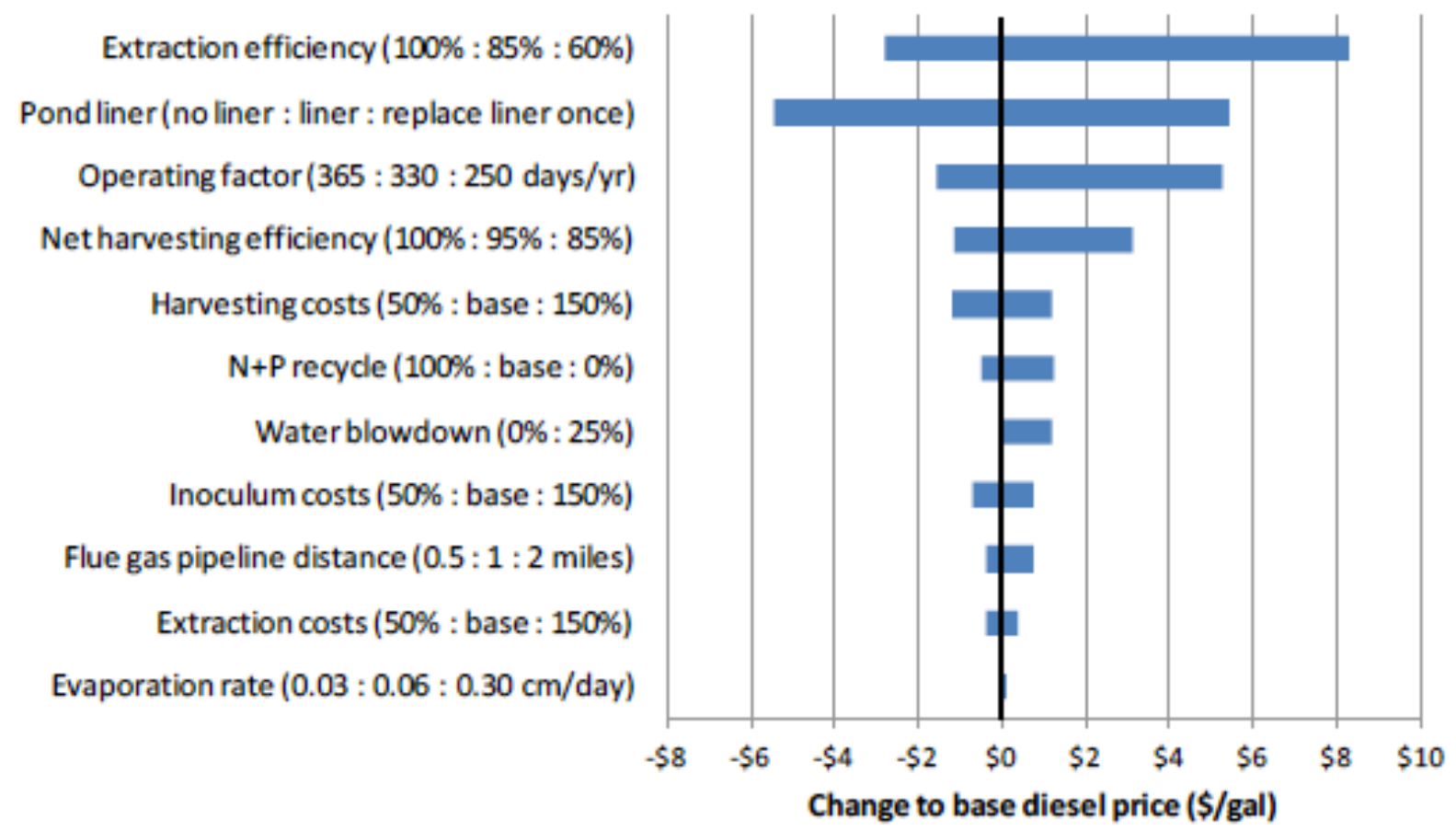

Figure 9 - Cost Sensitivity Tornado Plot for Key CAPEX and OPEX factors (ANL, PNNL, NREL, 2012).

\subsubsection{Raise Life Cycle Performance}

One of the primary objectives of this study is to investigate the commercialization potential of AJF from a LCA perspective and explore whether a carbon price framework can motivate investors to commit capital in the AJF value chain that is not currently profitable to invest in. Not surprisingly there is a correlation between rising carbon tax levels and attractiveness of investment in AJF but the desired commercialization effect is elicited only at tax levels greater than $\$ 1160 / \mathrm{tCO}_{2} \mathrm{e}$. Researchers and producers can augment the commercialization potential of AJF at more practical carbon tax levels by targeting lower life cycle emissions profiles so as to maximize the emissions benefit of AJF over PJF.

The LCA of AJF constitutes two stages, the WTP stage encompassing the emission transactions during the AJF production, and the PTWa stage capturing the emissions released during combustion of the fuel in the aircraft. The WTP stage is the carbon negative stage as the algae sequesters atmospheric $\mathrm{CO}_{2}$ during its growth phase. Although subsequent processing steps require energy and nutrient consumption that discount some of the captured carbon credits, GREET analysis indicates that the sequestration stage is generally strong enough to maintain a net WTP emissions negative balance. Conversely the PTWa stage is net carbon positive releasing the $\mathrm{CO}_{2}$ sequestered in the algal oil back into the atmosphere. This combustion stage is penalized by a carbon tax; technical improvements in engine manufacturing and advanced aircraft designs can mitigate the emissions profile of the fuel consumed to a certain extent but the large proportion of emissions reduction can 
be expected from improvements in the WTP stage specifically in the algal oil production regime.

\section{WTP Stage}

Similar to enhancing capital efficiency, developing optimal algal strains can aid in AJF emission reduction. A low resource intensive strain will relieve the emissions load of nutrient manufacture and operational loads of transport; in fact once strains capable of converting waste and recycled streams directly into excreted fuels are developed, lifecycle emissions will fall dramatically. But until R\&D can facilitate this transition, there are a number of areas researchers and producers can aim for in the short-term.

The NREL harmonization study proposes a number of system configurations to minimize the loss of carbon sequestered during the algal growth phase. The first is to lower the paddlewheel power consumption; pond mixing to maintain algal suspension accounts for much for the operational power requirements of the algal oil production facility, which depending on the source of electricity generation can entail an emissions footprint. As with much of the process schematic, the paddlewheel design and performance data is borrowed from non-fuel related commercial algal operations that does not prioritize emissions as an important performance criterion. The baseline harmonized model assumes a power consumption of $45 \mathrm{KWh} / \mathrm{ha}$ /day but newer alternative pond-paddle configurations combined with optimized paddle designs can potentially reduce consumption by up to $84 \%$ (ANL et al, 2012). Secondly more robust standards and technology utilization can reduce methane leakage from $\mathrm{AD}$ by ten fold if standards common to the natural gas industry are embraced. Methane, the main constitute of biogas, has very high global warming potential and exacerbates the carbon cost of AJF. The baseline model assumes fugitive emissions of $2 \%$ instead of targeting leakage below $0.2 \%$ (as in the natural gas industry). One avenue to ameliorate process design to reflect better performance is through replacement of the AD with Catalytic Hydrothermal Gasification (CHG), a catalytic wet process that converts the carbon and nitrogen in organic materials to methane and ammonia. A CHG unit "is compact, fast and hermetic, while AD requires large volume and long residence times. It is plausible that this technology will lower fugitive emissions rate to $0.2 \%$ while offering a $90 \%$ recovery rate for both nitrogen and phosphorus" (ANL et al, 2012, pg. 54). Lastly, the baseline scenario assumed that the net $\mathrm{CO}_{2}$ given off by the CHP process (powers facility by combusting natural gas produced from $\mathrm{AD}$ ) is cycled back to the growth ponds to supplement carbon content in the growth medium. However because the CHP flue gas comprises very low $\mathrm{CO}_{2}$ concentration at high temperatures, a large amount of energy is required to spar sufficient volumes of flue gas into the growth ponds as well as to overcome the dilapidating effect the elevated flue gas temperature has on blower efficiency (ANL et al, 2012). Alternatively, the flue gas can be discarded all together but unless equivalent emissions savings are generated in other areas of the production regime, the option will have negative consequences on lifecycle emissions balance (ANL et al, 2012).

It is important to acknowledge that under the baseline algal oil production regime, there is an inadvertent 
tradeoff between the lifecycle and technoeconomic performance due to counteracting forces prevalent during the winter season. Algal productivity is poor ( $3-6 \mathrm{~g} / \mathrm{m}^{2} /$ day) during the colder months when solar incidence is low which pushes up COP of algal oil and consequently the per unit seasonal GHG emissions; in the case of algal biodiesel, the winter GHG per unit fuel can exceed lifecycle emission from petroleum diesel. To reach a more acceptable lifecycle performance necessitates the facility be shut down during low productivity months. However modeling for a winter shut down scenario highlights opposing pressures to keep the facility running as long as the ponds do not freeze. This is because the variable operating cost, by definition the only costs which vary throughout the production cycle, represents a relatively small fraction of the final COP - roughly $11 \%$. The small saving does not outweigh the cost of idling expensive capital assets and the revenue lost from absence of product sales during this time. A possible remedy to this conundrum can be attained from continuing focused R\&D. Even a modest increase from baseline oil fraction from $25 \%$ to $30 \%$ by weight can improve system stability substantially and alleviate counteracting LCA and TEA pressures. Alternatively, facilities can switch production modes towards co-products during anticipated periods of low productivity to facilitate sequestration of carbon into a wider portfolio of products. Additionally, the LCA and technoeconomic performance may become easier to consolidate if and when a carbon price integrates emissions cost into the TEA (ANL et al, 2012).

\subsubsection{Generate Coproducts}

Co-products from AJF production will play a critical role in its commercialization because of the diverse portfolio of intermediate coproducts possible. Algal biomass like other plant biomass is a complex mixture of cellulose, hemicellulose, starch, lignin as well as lipids and proteins but the complex mixture can be separated into distinct high value product streams by removing inhibitors and contaminants that interfere with downstream processing, (O'Connell \& Haritos, 2010). Coproducts can provide additional revenue sources as well as mitigate emissions by directing the sequestered carbon in the algal biomass to other non-combustible carbonaceous compounds i.e. distribution across diverse product platforms displaces compounds that would otherwise be manufactured from fossil fuel or virgin materials. Algal oil processing gives rise to a number of valuable commodities such as nutraceuticals, pharmaceuticals, cosmetics, and animal feed along with a slew of low value intermediates (Taylor et al, 2013). Many of these coproducts can be sold in existing markets. For example lignin derived as a byproduct of $2^{\text {nd }}$ generation feedstock processing is used as a dispersant, filler and dust binder for road materials (Taylor et al, 2013).

It is a common practice in current commercial non-fuel algal operations to sell the Lipid Extracted Algae (LEA) to animal/fish feed markets (feed prices can be as high as $\$ 350 /$ ton) instead of passing the residual biomass into an AD for biogas. This may not be a straightforward option for algal cultivation for fuel for a number of reasons. Redirecting LEA from AD incurs an obvious loss in carbon credits and unless equal 
savings are captured somewhere else in the process, the overall LCA balance will be affected. Secondly, the wide price range of animal/fish feed will make it difficult to project the revenue stream expected from the sale of the coproducts and therefore despite the lucrative feed market, the potential returns may not be obvious. Lastly, tweaking process steps to generate animal/fish feed along with algal oil will place process limitations upstream in the algal oil production pathway. For example, the use of alum or other inorganic flocculants during the dewatering stage opens scope for flocculant contamination of the final dewatered LEA biomass. The present baseline algal oil production schematic uses chitosan as a flocculant for its biodegradability under anaerobic digestion. Chitosan might relieve toxicity concerns if animal/fish feed were to be produced but using it will require extensive validation from feeding trials before feed market adoption can occur (ANL et al, 2012). Furthermore in order to avoid contamination from solvent aided extraction, algal biomass will have to undergo mechanical grinding and gravity assisted separation to produce pure byproduct stream (Taylor et al, 2013). A possible production schematic can include a natural gas heated drum dryer instead of an $\mathrm{AD}$ to dry the LEA material prior to shipment to feed market but once again, removing the $\mathrm{AD}$ will negatively affect the lifecycle emission balance by preclusion of nutrient recycling, onsite energy production, emissions from methane leakage and low dryer efficiency (ANL et al, 2012).

A critical benefit imparted by coproducts that is often overlooked in TEA literature stems from the fact that unlike sectors like the pharmaceutical industry, where composition of matter patents are premium drivers of a company's valuation or profitability, biofuel producers are unable to capitalize on any patentable product due to strict and uniform end-use standards that all fuel products must meet. Instead industrial-scale biofuel manufacturing companies rely on novel processes that can efficiently convert accessible feedstock into fuels. "These companies compete on price, and thus on incremental innovation, manufacturing learning curves, cost of goods sold and logistic networks including robust supply chains of primary raw materials. The competition for marketing such commodity chemicals is severe, as it includes not only biofuels manufactured in countries with operational cost advantages but also conventional combustion engine fuels manufactured by conventional petrochemical companies. Moreover, the threat of product substitutes faced by biofuel manufacturing companies can expected to be exacerbated in the future by emerging modes of transportation motoring, such as electric engines or hydrogen fuel cells" (Vertes et al, 2010, pg. 541). But if and when a new line of coproducts and biochemical intermediates are established, it will allow biofuel producers to make proprietary claims as innovations in the fuel space that can be successfully leveraged as valuable intellectual property in the biomaterial arena. Novel molecules will enable new start-up biofuel companies to operate around a business proposition and an operational structure created for it, providing a nascent company with the ability to attract an adequate amount of investment (Vertes et al, 2010).

With the possibility of a multiple process options targeting a number of possible coproducts, comes inadvertently the question of what the ideal configuration of an algal biorefinery should be. Should it be a product-based or energy-oriented biorefinery? Or should it be a hybrid capable of both? Algal feedstock can 
be refined in most of integrated biorefinery designs that are operating today (or on the drawing board), hosting sufficient process flexibility to switch production modes between algal oils, carbohydrates or proteins. For now the pursuit to commercialize AJF seems to be more focused on the less developed dimension of the AJF production pathway - identification of better algal species and COP reduction from higher oil yields. Algal fuels are currently an order of magnitude costlier than other biofuel production pathways (Singh \& Gu, 2010). The market sustainability of targeted co-products must be considered in the context of their envisioned commercial production volume. At the vast production quantities associated with the fuel market, it may not be easy to find a value-added coproduct with comparable scale initially but, in the near-term at the level of a 39.31 MLY production facility, co-products could drive the economic feasibility of AJF (Davis et al, 2011).

\subsubsection{Further Considerations}

Producing AJF is only one stage of the process to creating sustainable jetfuel that can compete with PJF on an economic basis. Refineries, where downstream processing of raw algal oil takes place, are naturally the gateway for the AJF value chain as they stand between raw feedstock and readily usable end-use product. Refiners rely heavily on economies of scale and unless a large enough refining capacity exist to handle the volume of algal feedstock produced, AJF is unlikely to reach markets at a competitive price. Therefore it is essential that adequate refining capacity follow the scale up of advanced biofuel feedstock. However the drawback of building excess refining capacity is that sufficiently cheaply-cultivated biomass must be available in the immediate vicinity of the plant to justify building the large plants and minimize costs and emissions associated with feedstock transport (O'Connell \& Haritos, 2010). Refinery locational decision are made within the wider context of strategic, tactical and operational decision making process, integrating considerations for AJF production technologies, long-term demand-supply contracts, medium-term sourcing and logistics, and short-term market feedback. The cost-effective downsizing of technology to match smaller AJF value chains is an important R\&D goal in this area (Awudu \& Zhang, 2012).

While sufficient integrated biorefinery capacity is built, in the short-term algal oil feedstock is likely to be transported from production facilities to existing conventional petroleum refineries that are retrofitted with bio-feedstock processing infrastructure (CSIRO, 2011). Once online, newer integrated biorefineries will eliminate need to stockpile and allow for greater flexibility and risk-reduction around feedstock supply. Although refineries for competing biofuel pathways like Fischer-Tropps (standard technique to convert any solid feedstock to biofuels) are already available, HEFA facilities that are required to be built up from a zero base will also be built in the least amount of time due to their low capital intensity (CSIRO Data Modeling, 2011). On the other hand, mixed feedstocks also pose challenges for any single conversion process due to the variable chemical composition and contaminants characteristic of different bio-feedstock. Moreover, a versatile biorefinery will also have to juggle multiple harvest and delivery schedules making the refining 
activity concomitantly more complex as more diverse biomass sources are accommodated (Awudu \& Zhang, 2012).

\subsection{POLICY RECOMMENDATIONS}

There is resurgence in interest in new generations of biofuel technology underpinned by public and private sector R\&D activity and policy interest in enhancing the market penetration of biofuels. Notwithstanding this, the biofuel regulatory landscape remains dynamic (Vertes et al, 2010). Governmental programs such as emissions legislation, subsidies or tax incentives affect investment decisions between different technologies, support at specific stages of an innovation's lifecycle, and indicators of financial success (Vertes et al, 2010). Government policy can play an important role in creating market demand for RETs like AJF aligning it with a RET development roadmap. A rapidly growing demand plays two key roles in stimulating innovation. First, it accelerates private sector investment in innovation and instigates rapid diffusion of new technologies. Second, and perhaps more subtly, growing demand provides an important opportunity for immediate feedback from the market, whereby innovations are "more directly responsive to real market needs and less likely to fall prey to the isolation of the ivory tower" (Newell, 2010, pg. 258).

RET innovations respond rapidly to changing energy prices and government regulation as illustrated by patent data. One study noted that more than half of the full effect of an energy price rise on RET patent applications was experienced after just 5 years (Popp, 2002). This time frame takes into account the quality of knowledge available to private actors as well as public incentives for RET research (Newell, 2010). However, commercialization of innovation in highly regulated sectors like aviation often takes a very

long time. "In order to bridge the "valley of death", the gap between discoveries in the lab and the large-scale deployment of commercial products based on an invention, governments can help establish niche markets that reduce barriers and enable cost reduction and improved performance through learning by doing, learning by using, economies of scale, and network externalities" (Azar \& Sanden, 2011, pg. 137).

The following sections elaborate on some of the key actions that should be considered in designing a policy path for the commercialization of AJF.

\subsubsection{Compliment Carbon Tax with Other Policy Measures}

A carbon tax is commonly implemented with the hope of achieving two main objectives - to mitigate the source of carbon, and trigger the development and adoption of low emission technologies. A carbon price specifically guides the uptake of the most cost-effective low emission technologies by sending a consistent financial signal to consumers in combination with a demand-driven, profit-based incentive for the 
private sector to invest in continuous innovation (Newell, 2010). From a policy perspective a carbon tax is essential to stimulate the mass adoption of biofuels above its current share in the global energy mix of only around 2\% on an energy equivalent basis) (Timilsina et al, 2011). However from an investor's perspective a carbon tax (at any practical level) alone cannot promote AJF commercialization unless parallel policy instruments are put in place to complement the effects of the carbon tax. Without any new emission policy initiative, biofuels can be expected to account for only 5.5\% of total liquid fuels used globally by 2020 - about twice the current share. If a carbon tax of $\$ 25 / \mathrm{tCO}_{2} \mathrm{e}$ is implemented in isolation, the global market share of biofuels will rise to 5.6\%; a relatively high carbon tax rate of $\$ 100 / \mathrm{tCO} 2 \mathrm{e}$ will increase this to only $6.1 \%$ (Timilsina et al, 2011). When a carbon tax regime is aligned with a mechanism that directly targets biofuel producers and consumers using subsidies, market penetration of biofuels increases substantially.

For instance, a $25 \%$ subsidy applied in association with a $\$ 25 / \mathrm{tCO}_{2} \mathrm{e}$ will increase global biofuel penetration to more than $8.5 \%$ by 2020 while a $50 \%$ subsidy will carve out more than $15 \%$ of global market share for biofuels. Moreover, biofuel market penetration is more sensitive to subsidy levels than carbon tax levels. For example, a US $\$ 10 / \mathrm{tCO}_{2} \mathrm{e}$ carbon tax with a $25 \%$ subsidy will increase global biofuel penetration to $8.6 \%$ but if the carbon tax rate is raised to $\$ 25 / \mathrm{tCO}_{2} \mathrm{e}$ with the same level of subsidy, the global penetration of biofuels increases to only $8.8 \%$ (Timilsina et al, 2011). However aligning a subsidy with a carbon tax can be difficult without disclosing the source of revenue used to fund the subsidies. Admittedly a subsidy funded from the carbon tax revenue will be hard to justify because it will penalize producers and consumers of conventional carbon intensive fuels while unfairly favoring cleaner technologies (Timilsina et al, 2011). In the case of B.C.'s carbon tax framework, the province has designed its carbon tax as a revenue neutral instrument, meaning that all revenues collected from the tax is eventually returned to citizens and corporations through means of income tax credits (BC, 2012).

Opponents of the carbon tax claim that it raises prices of products and is an impediment on the competiveness of energy intensive industries which ultimately impacts economic growth negatively (Lin \& Li, 2011). It is argued that although a carbon tax may increase the relative output of the biofuel sector in comparison to outputs of most other sectors, the increase in output will be insignificant compared to the decrease in outputs from other sectors; a $\$ 25 / \mathrm{tCO}_{2}$ e carbon tax will increase output of the biofuel sector by $\$ 2.2$ billion by 2020 but also impose a cost of $\$ 1173$ billion worth of outputs from the other sectors (Timilsina et al, 2011). Moreover, a carbon tax is a also controversial because its intended impacts remain hotly debated across many jurisdiction that have implemented the measure; the wide-ranging reports of carbon tax impacts arise from different carbon tax rates, varying extent of tax exemption, as well as how carbon tax revenue was spent. In order to limit the impact on economic competitiveness, Sweden, Norway, Netherlands and Denmark provide tax exemptions for their prized manufacturing and energy intensive industries. In Norway, the rapid growth of $\mathrm{CO}_{2}$ emissions in the domestic oil drilling and natural exploitation sectors is compounded with the limited substitutability of remaining fossil fuel uses with renewable energy sources (hydropower, the main source of renewable energy has already reached the peak), ultimately negating most of the mitigation effects 
of the carbon tax. Denmark and Netherlands show that recycling carbon tax revenue to enterprises for environmental purposes can compensate for the costs imposed by tax exemption to some degree. In China, carbon tax exemption schemes indicate that in the scenario with no tax exemption or tax revenue redistribution, a carbon tax may have a greatly negative impact on GDP as well as the energy intensive industries (Lin \& Li, 2011). Mixed results arising from varying contextual settings and absence of any comprehensive empirical analysis (Lin \& Li, 2011) makes it very difficult to elucidate the effectiveness of a carbon tax (Knight et al, 2011). B.C. concedes that since the carbon tax was only established in 2007 and reached final tax level set in 2012, it is still too early to fully distinguish the impact of the measure from other influential factors in the economy such as 2008's economic downturn which lowered emissions and skewed the mitigation impact of the carbon tax in isolation (B.C., 2012).

Having said that, with regards to stimulating a novel technology like AJF, emissions policy can be designed to recognize the three primary affects emissions policy can have on innovation "(i) an abatement cost effect, reflecting the extent to which innovation reduces the costs of pollution control; (ii) an imitation effect, which weakens innovation incentives owing to imperfect appropriability; and (iii) an emissions payment effect, which can weaken incentives if innovation reduces firms' payments for residual emissions. The relative strength of the effects vary across policy instruments and particular applications, with no instrument clearly dominating in all applications" (Newell, 2010, pg. 259). The literature also suggests that carbon tax should be complemented by non-price fiscal instruments to reduce barriers to private investment and in particular aid innovators in overcoming early-stage market failures (Knight et al, 2011). A carbon price is technology agnostic and tends to favor later stage technologies which have already reached commercial scale. For early stage technologies however, alternative fiscal support through technology-specific feed-in tariffs, direct investment, tax exemptions, accelerated depreciation rates and renewable energy standards, can reduce CAPEX and guarantee an end market for the innovation. Without these measures, RET innovations may struggle to compete with more established RETs (Knight et al, 2011).

\subsubsection{Focus on R\&D Policy}

One of the conclusions reached repeatedly in this study is the importance of drastically improving algal oil content and productivity to generate AJF at a COP that will allow market adoption. Selecting and cultivating algal strains designed through advanced biotechnological tools for optimal oil production can offer higher yields. Current methods do not provide the scientific sophistication needed to grow the engineered algal strain outside lab settings but it is not hard to imagine that through continuous focused R\&D such limitations will eventually be overcome. In fact, since the beginning of aviation biofuel testing culminating in the ASTM approval of HEFA feedstock in 2011, the prices for a variety of biofuel pathways including algae have already dropped significantly (ATAG, 2009). The key parameter to propel AJF into a mainstream aviation use is time - when can AJF reach this stage? Since focused R\&D requires both time and capital investment, 
funding such activity becomes a question of creating the right environment to mobilize the financial commitment requisite to triggering innovation. The concept of induced innovation recognizes that R\&D is a profit-focused exercise motivated by the prices that innovations can attract (Newell, 2010). Governments have a big role to play in creating the context conducive to breakthroughs by setting policy directives that leverage regional natural resources and social-political objectives. If conditions exist but the technologies to complement this advantage are immature, governments can subsidize R\&D activity into certain technologies directly (Knight et al, 2011). Direct government support can fill the gap traditionally left by underinvestment by the private sector due to the limited profit potential of basic and applied R\&D. Unlike private enterprise; governments are in a position to consider social returns when making investment decisions. Government support of basic $R \& D$ is particularly important, as long-term payoffs, greater uncertainty, and the lack of a finished product at the end all make it difficult for private firms to appropriate the returns of basic R\&D (Newell, 2010).

Governments should also recognize that environmental and technology policies work best in tandem because while R\&D policy can help facilitate the creation of innovative RETs, it leaves little incentive for their market adoption. Supporting mechanisms for emissions control such as a carbon tax can encourage adoption innovations branching from publicly funded R\&D. In isolation, R\&D policy or carbon tax may not provide the welfare generated from their combined implementation; a carbon tax will only achieve $95 \%$ of the welfare gains of the combined policy, while a policy using only the optimal R\&D subsidy will attain just $11 \%$ of the welfare gains of the combined policy (Newell, 2010).

Investors generally regard government supported R\&D programs well and rank them equally as subsidies for manufacturing facilities or capital grants for SMEs or communities (Burer \& Wustenhagen, 2009). The same anecdotal evidence also suggests that investors seem to have very little faith in dedicated policy measures that directly support RET through short-lived subsidies. Investors seem much more sensitive to the technical feasibility or the proven performance record of a technology and thus prefer support for R\&D programs and demonstration projects (Masini \& Menichetti, 2013).

\subsubsection{Implement Agnostic Support Mechanisms}

Biofuels are only one set of RETs and within biofuels; there are many kind of biofuel technology pathways encompassing a variety of feedstocks, conversion processes and stakeholders. Currently, the ground transportation sector is the primary consumer of biofuels, its growing appetite for biodiesel and bioethanol accounting in large part for the huge proliferation of biofuel production and consumption in the last decade. The mass adoption of biofuels in this sector has been prompted by targeted government policies that have created efficient value chains from feedstock cultivation, refining and distribution via regulatory and financial 
subsidies. But as biofuel consumption permeates into other end-use markets such as aviation creating competing pressures on the limited biomass feedstock currently available, policy makers have to evaluate the effectiveness of existing support mechanisms to ensure equitable access to feedstock so as to not disadvantage the growth of newer biofuel markets. This brings into focus the importance of technology agnostic climate change related policies, typically referring to legislation that promote low carbon technologies without specifying particular attributes of the technologies to be supported (Azar \& Sanden, 2011). Policy makers usually support a variety of emerging technologies in order to avoid pitfalls or risks inherent to any one technology; making special concessions for any one subset or sub-technology pathways creates asymmetrical market incentives which can eventually impede the development of better but more immature innovations. But "governments often struggle with the idea of 'technology neutrality'. The EU commission often expresses support for 'technology-neutral' policies, but once rhetoric translates into action, technology-specific policies are (often) implemented. For instance, EU has not only issued a directive to promote the use of biofuels but also provides special support systems for wood-based biofuels. The policymakers understand that grain-based ethanol is currently more cost-effective" (Azar \& Sanden, 2011, pg. 138). However this approach must be balanced with the need to push forward the next generation novel biofuel conversion pathways (Azar \& Sanden, 2011).

\subsubsection{Subsidize Refiners}

Aviation, despite being a global industry, is only one small portion of the global transportation network. Road transportation dominates this sector consuming $71 \%$ of all transportation fuel leaving only $11 \%$ for by aviation (CSIRO, 2011). In terms of refinery output, since 2001 production of jetfuel has fluctuated between $6-6.3 \%$ of all petroleum derivatives produced (Nygren et al, 2009). Due to the chemical composition of crude oil and jetfuel hydrocarbon chains being shorter than diesel range hydrocarbons, refiners have a natural ability to produce both derivatives, switching production modes to reflect market demand for diesel or jetfuel. But generally, refiners have a stronger incentive to produce more road transportation fuel than jetfuel due to the higher operating costs incurred and lower revenues received from jetfuel production. The additional cost arises from hydrogen production (from natural gas) and revenues foregone due to generating lower quantities of higher-value diesel fuel (Pearlson et al, 2013). This is true for refiners producing biofuels as well as conventional refineries that have upgraded their capacity to process vegetable oils (ATAG, 2009). But policy measures generally provide subsidies for road biofuel producers - the US Volumetric Ethanol Excise Tax Credit provides $\$ 0.13 / 1$ to ethanol producers and the American Jobs Creation Act offers biodiesel producers $\$ 0.26 / 1$ (Sorda et al, 2010) - while no current direct incentives exist to sway more production to bioderived jetfuels (PARTNER, 2013). Given that vegetable oils can be converted to road biodiesel via a triedand-tested process called transesterification at a cost of up to $\$ 0.15-\$ 0.20 / 1$ less than the COP of bioderived jetfuel (CSIRO, 2011), refiners who choose to produce the latter suffer a strategic disadvantage (Vertes et al, 
2010) i.e. a typical refinery processing AJF will incur higher COR and have less money to acquire scarcely available feedstock.

In the long-term competition between the road sector and aviation industry will ease due to the gradual electrification of road transportation, ramp up of feedstock production and certification of multiple conversion technologies. But unless short term policy measures recognize the disparity in access to scarce biomass feedstock, ongoing excise arrangements and relative cost of feedstock refining technologies between road and aviation fuels, development of newer technologies like AJF will be impeded.

\subsubsection{Implement National Mandates}

National targets play an important role in setting the direction and aspirations of climate change related policies. They can either be mandatory - supported by legislative penalties and incentives - or merely aspirational. Although targets do not directly offer certainty, they ensure private investors of a sizable future biofuel market. In the absence of aviation fuel blending targets (mandate requiring all jetfuel to have a minimum biofuel content), bio-SPKs must compete with PJF in the extremely price sensitive fuel market where COP tends to price Bio-SPKs out of the market (Knight et al, 2011). National targets have been key in establishing markets for $1^{\text {st }}$ generation biofuels. For example, the EU RED continues to stimulate demand for biofuels by stipulating national targets for member nations, defining biofuel use obligations, and defining a regulatory framework by which incentives are linked to the environmental performance of individual fuels, aiming to ensure the sustainability of biofuels consumed in the EU (Vertes et al, 2010). In Brazil, the largest producer of bioethanol in the world, the government mandates a $20-25 \%$ blend of ethanol with gasoline, sets lower taxes on ethanol storage, and protects the domestic producers with a $20 \%$ duty on imports. Equivalent support for alternative engine technologies has resulted in $83 \%$ of all cars sold in Brazil to be flex-fuel vehicle capable of running on $100 \%$ bioethanol (Sorda et al, 2010).

In order to make AJF equally attractive, long-term stable policy regimes built on sound legal foundations (like feed-in-tariff programs prevalent in other RET sectors) are essential to attracting the serious private sector investment needed for it's continuing development (ATAG, 2009). As with R\&D and emissions policy, it is important that decision makers implement measures in a staged fashion to ensure that newer technologies do not arise prematurely before previous policy measures have created conditions for its success. For example, in the case of coal powered electricity generation, introducing a carbon tax in the absence of earlier stage policies poses the risk that carbon price inflation will be borne fully by electricity consumers because of a lack of viable energy alternatives. The strategic staging of related policies can ensure that a constant pipeline of new clean energy technologies will emerge to compete well with coal-fired electricity, pushed to even greater success once a carbon price arrives (Knight et al, 2011). The duration of implementation of 
policy instrument in questions is also an important consideration, as well-targeted policy meant to inspire innovative solutions should ideally aim to be temporary; its should be reduced and/or removed once solutions diffuse into the market in order to avoid dependency on policy incentives (Knight et al, 2011).

\subsubsection{Implement Emission Standards}

Unlike road transportation fuels, aviation fuels are typically precluded from key renewable energy policies. In the US, the EISA mandates the consumption of at least 32 billion gallons of biofuels by 2022 without specifying any expectation for the aviation sector. The RFS does offer producers tradable credits based on the renewable content should they produce Bio-SPKs, but for reasons discussed, without mandating aviation specific targets most feedstock acquisition and biofuel production will fulfill the demands of the incumbent industry. The EU's Emissions Trading Scheme (ETS) recently attempted to include aviation under its umbrella, charging airlines flying into EU airspace a carbon tax based on jurisdictional emissions. Airlines are eligible for exemption from this tax if aircrafts operate using biofuels that are catalogued by the EU and that align with its RED criteria for sustainability. But the aviation component of the EU ETS is currently inactive following multiple complaints and high-profile trade threats made by affected parties (Economist, 2012). The contentious policy measure highlights two major barriers in including aviation in climate change related policies. Firstly aviation, being a cross-boundary operation, faces significant compliance hurdles as airline operators are required to conform to multiple regulatory standards that carry varying stipulations for the eligibility of biofuels for incentives or mandates. Secondly, biofuel directives are often tied to their emissions profile but the lack of a universal framework disallows universal verification emissions performance and enforcement of emissions standards. Both the EU RED and US RFS differ in the methodologies applied. Under such a scenario "a biofuel producer must decide if its bio-jetfuel should be certified for sustainability according to the regulations of the country of production, departure, or arrival. If a biofuel producer in Brazil exports bio-jetfuel to the US where it is delivered to an airline for a flight to the EU, Brazilian legislation is applicable to the production, US RFS is applicable if the importer wants to obtain tradable certificates (Renewable Identification Numbers - RINs) and finally the EU RED requirements have to be met if the airline applies for exemption from the EU ETS...Moreover, biomass and biofuel producers will be led to sell preferentially into those countries which offer the highest incentives making it difficult for other countries to acquire the limited supplies of bio-jetfuel" (IATA, 2012, pg. 16). In order to avoid costly multiple certifications and stimulate global uptake of Bio-SPKs including AJF, cross-border regulations and related biofuel accounting schemes have to be harmonized.

A globally harmonized sustainability criteria for biofuels will need to be built on universal methodologies capable of cataloguing and classifying biofuels derived from feedstock grown under diverse natural and process conditions, in addition to elucidating lifecycle emissions associated with land-use change. Although 
understanding the magnitude of carbon released during land-use changes remains a topic of much debate, sufficient information is available to warrant a precautionary approach before any biofuel produced from carbon rich inputs is produced (Malaysian biofuel producers are notorious for clear cutting rainforests to cultivate palm). Studies indicate that the potential magnitude of carbon released from consumption of feedstocks like palm oil is sufficiently large to negate any emissions credits. In contrast, algae derived biofuels perform substantially better especially if cultivated in non-arable or decrepit land which elicits negligible land-use change emissions (RAND, 2009). An emissions policy framework built from mutual recognition of sustainability certifications between different jurisdictions is therefore strongly likely to favor AJF (IATA, 2012).

An additional consideration for policy makers to explore in the short term to vitalize AJF while a robust international emissions framework is devised is an Ultralow-Sulfur specification (ULS) for aviation fuel. Advanced biofuels made from vegetable oils contain zero traces of sulfur, an attribute that translates into negligible $\mathrm{SO}_{\mathrm{x}}$ emissions during combustion. An ULS specification will align aviation with specification existing for highway diesel fuel which will indirectly impart benefits upstream in the supply chain by potentially improving refinery scheduling and operations, and encourage multiuse pipeline operations. An ULS jetfuel specification will ease the introduction of HEFA fuels into the commercial liquid transportation fuel infrastructure (along with crude oil derivatives) as HEFA fuels pose similar infrastructural compatibility concerns such as lubricity, effect on seals due to low sulfur, and reduced aromatic content. Given the humanhealth impact of particulate matter (PM) and gaseous PM precursors arising from fuel combustion during takeoff, landing, and ground operations, ULS jet fuel will virtually eliminate primary and secondary PM generated by aviation emissions. Unlike new aircraft and engine technologies that take some time to diffuse into and airline fleet, the air-quality benefits of sulfur elimination will be realized as soon as ULS jetfuel is introduced (RAND, 2009). An ULS specification can be easily implemented and enforced in the industry because the process of removing sulfur from fuel derivatives is well understood and is widely practiced by refiners wherever ULS diesel standards exist. Having said that, prior to implementation of an ULS specification, consideration will have to given to the adverse consequences of higher fuel cost associated with ULS jetfuel, an increase (of about 1\%) in the fuel volume purchased and consumed, a reduction (of about 1\%) in the aircraft range with full fuel tanks, an increase (of about 2\%) in life-cycle GHG emissions, and the elimination of sulfur aerosols which have a short-term climate cooling effect (RAND, 2009). 


\subsubsection{Model Technology Policy Around Investor Interests}

When designing technology-centered policies aiming to guide the development of new low carbon innovations, policy makers can take a technology-push or market-pull approach. Technology-push policies such as government-funded R\&D programs aim to increase the amount of technology 'supply' whereas market-pull policies such as public procurement of production tax credits target demand creation for new technologies, providing producers and consumers economic incentives to apply them. There is much deliberation amongst climate policy scientists and modelers about the optimal approach to reach long-term emissions mitigation targets, with proponents of technology push arguing for the need to allow breakthrough innovation to gain visibility to consumers, in contrast to market-pull instruments that aim to ensure a market for new technologies is created prior to their introduction (Burer \& Wustenhagen, 2009). Often governments compromise and pursue a mixture of both policy approaches.

The more practical challenge for policy makers lies in designing optimal programs to push new technologies from research laboratories to market through the 'technology valley of death' -the middle phase of the commercialization pathway where successful prototypes of the technology are developed but cannot progress further due to insufficient revenues funneled into continuing development (Burer \& Wustenhagen, 2009). Fortunately, experience shows that this is an area where private equity investors like Venture Capitalists (VCs) focus their commitments. Private investment in the renewable energy sector has recently surpassed $\$ 100$ billion per year, the largest contributions being in wind farms or biofuel projects. Early stage investors have historically played a crucial role in RET development and so the stage of the commercialization pathway where they are most active in, as well as the associated market conditions, naturally become good benchmarks to guide technology policy (Burer \& Wustenhagen, 2009).

In order to create an environment conducive to investment, policy should accompany support mechanisms that attract investor interest for biofuel projects. Renewable energy projects are typically characterized by high UCC; large 'front-loaded' capital investment is requisite to realizing the eventual benefits of generating renewable energy at relatively low OPEX. Bank financing is the primary source of funding for such capital intensive projects but traditionally securing backing from debt providers has been the main hurdle for renewable energy developers due to technological, operational and project-related risks elaborated earlier (Knight et al, 2011). Project finance lenders are traditionally conservative and tend to favor low-risk projects (even if prospect for high returns exist) involving tried-and-tested technologies that can provide returns on investment irrespective of the policy climate (Vertes et al, 2010). In the last decade, banks have become increasingly unwilling to lend to $1^{\text {st }}$ generation biofuel projects in response to the inefficient bioconversion process technologies that have come to dominate the industry and concerns surrounding the long-term sustainability of the primary feedstock. Although advanced feedstock and newer process pathways alleviate 
partly, or all of, the concerns associated with outdated $1^{\text {st }}$ generation pathways, lenders remain cautious. This perpetuates a paradoxical problem - financiers are unwilling to lend capital for new infrastructure until an adequate and robust value chain is secured but producers find it very difficult to commit resources until they are assured that the infrastructure to process and deliver their product to market exists (Vertes et al, 2010). Governments can interject to break this chicken-and-egg problem by legislating specific incentive structures for early stage R\&D activities, creating publicly funded VC funds, and partnering with private financiers to share risks in funding demonstrations projects for technology validation. Policy makers can also encourage formation of technology clusters. For AJF producers the business model represented by supply chains in the US corn-belt is a good model. Smaller projects targeting smaller markets should be optimally located near customers and local sources of primary raw materials, while larger projects should leverage not only their size advantage by being located in areas of highest biomass concentrations (synonymous to the US Corn Belt for corn ethanol production) "but also their ability to absorb larger due diligence and transaction costs. While these latter costs will decrease as lenders become more experienced with managing clean technology or biofuel project opportunities, the combination of various projects with different risk profiles may generate a portfolio effect that ultimately decreases the overall risk" (Vertes et al, 2010, pg. 533).

\subsubsection{Assimilate Considerations for Investor Psychology in Investment Policy}

Another strategy to develop informed technology policies that can motivate investment in AJF is to examine factors that impact investor perception of RETs and how in turn that perception translates to investment behaviors. Investors look upon RET investments favorably as an option to diversify their investment portfolio and decrease their overall portfolio risk. A recent stream of research studying RET cost contributions relative to risk contributions to a portfolio of energy generating resources (including fossil fuel sources), indicated that adding RETs decreased the overall portfolio cost and risk, even though the stand-alone generating costs of each RET was higher (Masini \& Menichetti, 2013). This is because by investing in a range of RETs investors can curtail the risk associated with sudden changes in exogenous factors (raw materials price and supply, regulatory framework or shift in consumer preferences) that may affect the viability of any one RET. Having said that, despite relying on the role of private finance to accelerate RET deployment, RET policy literature seldom incorporates investor perspective. Policy makers generally take a very rational approach in assimilating the technology performance, economics and market adoption efficiency with the underlying assumptions for private investor behaviors. "Yet, there is increasing evidence that a purely rational economic evaluation of the investment alternatives does not suffice to explain how investors deploy capital or how agents choose among competing energy technologies. An emerging stream of literature suggests that broader social and psychological considerations must be included in the analysis of energy systems" (Masini \& Menichetti, 2013, pg. 511). RET investors are likely to use three primary sources of information to make investment decisions, with their decisions varying with the extent of their sensitivity to the source of 
information: i) they observe the behavior of their peers (well known investors in the same industry), ii) they consider the opinion of external consultants who specialize in the RET industry, and iii) they use factual information originating either from technical reports or from due diligence conducted in-house. Following from these guidelines, it seems that the perceived technological feasibility (or lack thereof) of a RET project is one of the most significant barriers to RET investment which highlights the critical importance of technology validation projects early on (Masini \& Menichetti, 2013).

Secondly and more importantly, the economic viability of most RET projects is often dependent on the level of confidence investors have in the effectiveness of incentive mechanisms (Masini \& Menichetti, 2013). Public policies most often are built on uncertain grounds because they are subject to political ideology and public sentiment. This uncertainty have been identified as a powerful deterrent in securing private-sector investment as highlighted by the investment downturns following changing regulation in Denmark, in Germany and the US (Masini \& Menichetti, 2013). Given the usual time lag between initial discovery and profitable market penetration, it is critical that private investors feel confident of sufficient demand existing for their innovation once it reaches the market. An example of a consistent and stable regulatory framework that has been able to mobilize substantial private capital is the feed-in-tariff system that is very popular in Europe where it has allowed for large profitable corporate operations within the wind and solar energy sector. Investors respond well to feed-in-tariffs because they are encouraged by its long track record as a stable policy instrument especially in Germany (Burer \& Wustenhagen, 2009). However, although policy makers and industry are in general consensus that an economy wide carbon tax with increasingly stringent emissions targets set through several decades (Newell, 2010) is a good policy direction, early stage investors are not univocally positive due to the lock-in affect of long-term policy incentives. Investor behavior with regards to RET policy is one that can be best described as policy averse, meaning that investor decisions are evaluated without any regards or assumptions for policy incentives. Qualitative studies suggest that investors generally avoid long-term commitments in projects based solely on projected tax credits or subsidies. Investors prefer instead short-term 3-5 year subsidies to aid in the initial technology push after which the credits should expire to allow projects to stand independent of government support. As far as emission policies are concerned, cross-border investors express concern about the multitude of regulatory frameworks and mixed standards that exist across jurisdictions (Burer \& Wustenhagen, 2009).

Investor sentiments can also reflect the underlying regional socio-economic and cultural paradigms which can turn inform investor skepticism or optimism about the success of RETs. For example, large corporations in Europe tend to rank climate change as a key driver of the clean energy industry whereas American investors rank energy security and competitive advantage (followed by climate change policy) as key drivers. Moreover, U.S.-based investors also tend to be more skeptical in general about clean energy support schemes as a way of increasing investment in the clean energy sector and are likely to refer to market signals such as high oil prices as the key driver for their investment (Burer \& Wustenhagen, 2009). Public perception of 
climate change and the extent to which it is captured in policy can also motivate investor behaviors; highly eco-sophisticated populations largely prompt developed economies, such as those represented by the Organization of Economic Cooperation and Development (OECD), to pursue environmental oriented policies. Countries like Germany, Denmark, Ireland and Belgium, having access to relatively large physical resources of wind, solar, biofuel, or geothermal, have fostered RET industries in response to the perceived need to reduce the import of carbon heavy coal, oil and natural gas. Other countries like Iceland, Norway, New Zealand and Austria have historically relied on geothermal and large-scale hydropower and so are natural inclined towards RETs. These countries exhibit an innovation nirvana where efforts to commercialize (and sequentially innovate) RETs are met by "wide-spread acceptance and demand, limiting the economic risk and allowing for commercialization through the use of venture capital, equity investments, or through the acquisition of other innovation by larger incumbent energy firms" (Walsh, 2012, pg. 38). Other countries like Canada, Finland, Norway and Switzerland, which have access to low cost domestic electricity supply generated from large-scale hydro and/or nuclear stations generally exhibit higher sensitivity to fluctuating renewable energy demand, create economic risk for RET providers and trigger a more discontinuous innovation process. Consequently, these jurisdictions deploy more cautious commercialization strategies involving joint ventures or strategic alliances with large incumbent energy firms instead of equity investments (Walsh, 2012). In addition to eco-sophistication, the overall economic wellbeing of the region where RET investment and adoption is in question is also important as in the long term, increases in real GDP per capita and $\mathrm{CO}_{2}$ emissions per capita are found to be major drivers behind per capita renewable energy consumption. "Higher income individuals are more likely to have their basic needs and wants met and be concerned with environmental issues and have the money to invest in and purchase of renewable energy. Higher income countries are also more likely to have access to or the development of new technologies that are important to the increased production and use of renewable energy" (Sadorsky, 2009, 461). In such social context a $1 \%$ increase in real GDP per person can potentially increase per capital renewable energy consumption by $8.44 \%$. Similarly a $1 \%$ increase in $\mathrm{CO}_{2}$ emissions per person can potentially increase per capita renewable energy consumption by $5.23 \%$ (Sadorsky, 2009).

Taking into consideration the behavioral context (economic conditions, state of technology and historical trends) of private investors can be useful in determining the efficiency of policy making decisions across different socio-economic environment which can in turn inform the best line of strategies to deploy AJF. For example, developing economies such as India and China might appeal as potential jurisdictions for algal technology investment but because these regions have traditionally exhibited harsh realities for RET developers, policy frameworks borrowed from regions with high level of eco-sophistication and demand for renewable energy products might not be realistic unless the technology is so disruptive that its potential return outweighs the commercial risk. Rather, the framework applied in India or China should be one that encourages collaborative strategies between government, utilities and private investors. This way private investors "will save time and resources with a greater choice of successfully diffusing their innovation into 
the market. Investors will benefit from reducing their risk of investment through a better understanding of the RET environment they are investing in. Concurrently policy makers will also be able to judge what policies best motivate adoption of RET within their jurisdiction" (Walsh, 2012, pg. 40).

\subsubsection{Create Cross-Disciplinary and Cross-Regional Partnerships}

Over the next decade global air traffic ridership is expected to rise by $180 \%$ followed by a $140 \%$ growth in $\mathrm{CO}_{2}$ emissions - the lower emissions growth rate is mediated by a baseline technology improvement of $1 \%$ per year. However determining the expected passenger growth rate is not straightforward due to the uncertainty of how aviation and its passengers will treat any cost impacts of technological improvements or policy shifts. For example, if advanced engine design allows for greater fuel efficiency, the resulting cost savings may eventually be passed on to passengers in form of cheaper ticket prices, which will raise ridership, airline operations and inevitably negate any emissions mitigation effects of the fuel efficiency measure. Because of this uncertainty, aviation is choosing redundancy in its approach and support for both demand and supply-side measures that can allow the industry to maintain both forecasted growth rate and fulfill its emissions targets. Simulation studies hint that supply-side mechanisms, such as biofuel use, provide significantly more room for growth than demand management strategies, such as short-haul transport or price on carbon, while imposing comparable levels of emissions (Bonnefoy, 2011). Demand management schemes become effective only if aggressive measures are implemented. In fact a $\$ 200 / \mathrm{tCO}_{2} \mathrm{e}$ price will offer only a mere $8 \%$ emission reduction compared to the baseline scenario. Similarly, mitigating aviation emissions by $20 \%$ will call for diversion of up to $60 \%$ of all short-haul flights to other transport modes. In contrast the use of biofuels offers one of the more practical means by which policy measures and industry effort can have a measurable impact (Bonnefoy, 2011).

The promise of advanced biofuel technologies like AJF is still far from being met. There is a big role for government to play in strongly supporting lab-scale and pilot scale R\&D necessary to translate scientific innovations to commercial scale feasibility. It is possible that a commercially viable AJF technology pathway will emerge from research activity facilitated solely by the private sector but the very limited return on investment associated with early stage R\&D will make this unlikely. There is considerable room for development before aviation biofuels become economically viable but given the immediacy with which aviation has to start transitioning to a de-carbonization, stakeholders need to act now to form the networks and put in place actions to ease the transition when restrictive policies are eventually enacted (ATAG, 2009).

In the last decade biofuel production has been driven by major biofuel producing regions. The key instruments fostering production and increasing consumption have been mandatory blending targets, tax exemptions and subsidies. Governments have intervened in $1^{\text {st }}$ generation biofuel value chain by supporting 
intermediate inputs (feedstock crops), subsidizing value-adding factors (labor, capital, and land) and granting incentives that target end-products in addition to enforcing import tariffs that protect national industries from external competition (Sorda et al, 2010). Brazil has very successfully applied these strategies to leverage its natural resources to establish a local biofuel industry that thrives on its strong stable supply chain and low COP; relatively cheap sugarcane feedstock, high land productivity coupled with almost no needs for irrigation, use of biomass residue for co-generation (mills are able to satisfy almost all of their energy) and several years of governmental support mobilized large investment in R\&D and technology developments that have been critical elements of Brazils energy independence (Sorda et al, 2010).

Having said that producers and consumers need to inevitably move away from $1^{\text {st }}$ generation fuels to newer fuel technologies to ensure long-term sustainability. For aviation this is a critical requirement seeing that the sector is very likely to be married to liquid fuel technologies for the coming decades. But there are lessons to be drawn from policy conditions and industrial cooperation that have been characteristic of the last decade of biofuel proliferation. Similar to the stakeholder partnerships in the incumbent biofuel industry (that largely addresses the needs of road transportation industry), the aviation industry, government, AJF producers, agricultural sectors and academia should come together to combine expertise and analyze the opportunities for regional AJF production including the optimal feedstock cultivation techniques and infrastructure requirements (ATAG, 2009). Immediate industry stakeholders like airlines, airports, aircraft, engine manufacturers and fuel refiners can partner with academic institutions and agricultural companies to transfer knowledge an expertise to the facilitate development of advanced feedstocks. Extending the collaboration to local, regional and national government agencies, aviation regulators as well as environmental and sustainable development NGOs can further cement the stability of the value chain while also educating public perception so that the controversies encompassing $1^{\text {st }}$ generation biofuels do not impede the development of advanced biofuels (ATAG, 2009).

Policy makers need to level the playing field between road the transportation sector and aviation so that refiners are not unfairly motivated to produce road fuels over aviation fuel. For example under the current EU RED, 10\% of all energy in the road transport sector must be derived from renewable sources by 2020 and unless provisions for bioderived jetfuel are introduced, RED may cause a shift of all available sustainable biomass to road transportation biofuels (SAFUG, 2010). Over the long-term, the most profitable use of limited biomass resources will be to provide liquid fuels for transport services as competing uses will catered for by increasingly affordable solar and wind energy. Within the liquid fuel market the relative competitiveness and lower carbon intensity of electricity will likely push rail and urban short haul transport towards greater electrification, leaving long haul transport and aviation as the main users of Bio-SPKs (CSIRO, 2011). In the short-term, since only about $6 \%$ of global refiner output is allocated to jetfuel, and aviation does not have any immediate practical alternatives to liquid fuel, policy should recognize that unless prioritized, the emerging aviation biofuel market may collapse before having a chance to develop (SAFUG, 2010). 
The key focus of aviation and the investment community should be to build a case for sourcing investment funding and coordinating investments along the value chain. Actions that directly target components upstream of the supply chain will be of most value. Some other priorities are resource assessment including proximity to refining infrastructure, biomass production trials, and refining technology development (CSIRO, 2011); initiating a number of large-scale infrastructure projects will be critical in sparking confidence in industry and government. Currently Germany is building at least one large-scale HEFA refinery aiming to encourage industrial activity that will guard against a dependency on imports, utilize the domestic engineering capacity investment and remove barriers in the supply chain in the process (IATA, 2012). Aviation and government can also play an important role in supporting off-take agreements - an agreement between a producer and a buyer of a resource to purchase/sell portions of the producer's future production - with refiners for volumes of Bio-SPKs; off-take agreements are typically negotiated prior to the construction of a facility and offer confidence in the presence of a future market once the facility generates fuel. "Discussions with industry have revealed a desire for supply contracts with durations of a minimum of 10 years. The contracts and off-take agreements are vital for producers to gain financing from capital investors" (IATA, 2012). Off-take agreements will in turn support refiners in developing supply relationships with biomass producers (CSIRO, 2011) and aid in overcoming the 'chicken and egg' problem. For example, in the US, the Defense Logistics Agency (DLA) which purchases fuels for the Army, Navy, and Air Force, is currently certifying its aircrafts for bioderived fuels and financially committing to large orders (IATA, 2012). DLA is bridging the gap between certification and commercial scale production by supporting a variety of demonstration programs and initiatives through partnerships with other government organizations and industry. These efforts are helping promote a competitive industry while potentially providing the military a supplier base capable of producing operational volumes of sustainable fuel to meet future goals (IATA, 2012). These efforts are complemented by renewal and expansion of the RFS to include aviation biofuels (IATA, 2012) thereby offering producers and importers financial credit equal to the renewable content for advanced aviation biofuels. In Europe, an example of a prominent partnership is the Aviation Initiative for Renewable Energy in Germany (AIREG) which targets cost reduction in securing feedstock and fuel production by pushing for long-term agricultural financing arrangements with producers of energy crops including algae cultivators. Moreover, loan guarantees made available by the German development bank KfW and a national bio refining strategy are being implemented to ensure the currently pending transition from pilot plants to industrial production.The biorefinery strategy also provides investment grants for biorefineries that are accompanied by price guarantees for biofuel producers (IATA, 2012).

In the short term, governments should champion R\&D activity in a few key areas. Firstly, applied R\&D through international commercial and scientific partnerships should focus on bringing to fruition solutions for low yield and high cost attributes of current algal cultivation regimens (CSIRO, 2011). Although not exclusive to algae derived fuels, simulations hint that a $20 \%$ reduction in sustainable biomass COP can 
potentially accelerate establishment of a bioderived jetfuel industry in around five years (Graham et al., 2011) (CSIRO, 2011). Secondly, international scientific cooperation will need to address the need for a robust sustainability standard that provides a consistent and equitable process for airlines, passengers, and governments to assess biofuels for certification centered on sustainability criterion; a comprehensive sustainability criteria that ensures that a source of aviation biofuel does not displace food crops or elicit deforestation, has minimal environmental impacts, and a net positive socioeconomic impact on a region, will distinguish advanced biofuels and particularly AJF from competing sources, thereby encouraging market uptake. In the event that a cross-national carbon pricing framework is established, a comprehensive sustainability criteria will also make low emissions intensive biofuels like AJF more cost competitive and hence more profitable to produce (SAFUG, 2010). A harmonized standard will also provide for uniform enforcement across jurisdictions unlike now where a patchwork of standards elicit mixed incentives and inhibit the development of a commercially viable AJF market. As of now, the Roundtable for Sustainable Biofuels (RSB) - an international multi-stakeholder initiative that has developed a third-party certification system for biomaterials production and processing along environmental, social and economic criteria - is the best candidate for an effective harmonized system. 


\section{CONCLUSION}

According to one assessment, the period between 2007 and 2008 witnessed a $400 \%$ rise in investment in algal biofuels which according to the investigators is not surprising given the potential projects to yield an Internal Rate of Return (the DF at which the NPV is zero i.e. the higher the IRR, the better the investment prospect) of $15 \%$ and the future potential for investors to attain higher returns as biotechnology and process improvements are made (Stephens et al, 2010). But if this figure is reflective of investor attitude in the sector, then the obvious question is why have economically viable microalgal biofuel production systems not yet manifested. There are two possible explanation for this; first, existing pilot and demonstration plants are mostly smaller than 5 ha, well below the size threshold for economic viability, and second, "insufficient time has passed for the industry to evolve from recent capital injection through to large-scale commercial production. Thus, the most appropriate and cost-effective mix of technologies are yet to be successfully integrated and optimized, and even realistic, viable enterprises are still in the commercial development phase" (Stephens et al, 2010, pg. 127).

The timely commercialization of AJF will depend on the intersection of efforts undertaken by multiple stakeholders from biofuel producers, refiners, distributors to players further down the value chain from airplane manufacturers, engine manufacturers, airport owner(s), airplane operators, government, and even the community, both in the near vicinity of the airport and the algal farms (Kivits et al, 2010). To create a practical roadmap to bring AJF to the fuel market at competitive prices, technological prowess will have be complemented by legislative confidence in the technology that aid in leveling the playing field between advanced biofuels and more traditional biofuel that have dominated both feedstock acquisition and market share. The transition to sustainable aviation will be influenced by two external intermingling factors (i) the perceived need to change the aviation industry towards a more sustainable aviation paradigm (henceforth, the perceived need), and (ii) technological innovation. Here technological innovation is not just a push from advancements in AJF but also societal attitudes that dictate which innovations should be retained or discarded (Kivits et al, 2010). The current trajectory of technological change, characterized by a cautious industry and semi-concerned public, is one where investments are hedged through development activity in multiple technologies. Although this is not necessarily a bad thing, it does mean that it is more likely that significant financial resources will be misallocated to pursuing technologies that might not become commercially feasible (Kivits et al, 2010).

A primary focus for AJF investigators and producers in the coming decade will be in the feedstock production side investigating the optimization of not only the algal strains but also process technologies. For example although expensive now, advanced PBRs offer reprieve from the low productivity, susceptibility to contamination, and sub-optimal controls of open ponds; through continuous development PBRs are likely to become dominant cultivation technology (even in hybrid system) for industrial scale fuel operations 
(Knoshaug 2011). Furthermore, use of sophisticated PBR systems will also allow integration of other RETs as components that can enhance the energy return on energy input performance of the cultivation step and downstream process steps. For example, future algal oil production can be undertaken by integrating PBRs with Concentrated Solar Power (CSP) systems that can deliver electricity to supplement PBR requirements. Currently CSP systems can have CAPEX and OPEX that is twice that of conventional PBRs but again both technologies are to experience significant cost improvement in the coming years (Taylor et al, 2013). Additionally, biotechnological advancement that allow tailored algal strain to be used as contextual fuel production solution will allow both the leverage of regional resources and the potential integration of multiple markets that can provide substantial overall financial benefits. For example, if algal cultivation occurs near the coastline, the source of water to cultivate algae can also be self-supplied by connecting with water desalination plants. Additionally, the desalination plant would offer the flexibility of algal strain selection - producers can select freshwater or saline tolerant strains. Much of the water in the whole process can be recycled, and the excess water generated by the desalination plant can, in principle, be sold to local residential authorities (Taylor et al, 2013).

A second critical area of investigation for the coming years will be in emission science. A WTWa analysis usually captures the impact of non- $\mathrm{CO}_{2}$ emissions to a limited degree. But climate-relevant aviation emissions profile has a number of non- $\mathrm{CO}_{2}$ constituents including $\mathrm{NO}_{\mathrm{x}}$, sulfur compounds and un-combusted hydrocarbons that can interact with chemically active GHGs ozone $\left(\mathrm{O}_{3}\right)$ and methane $\left(\mathrm{CH}_{4}\right)(\mathrm{Krammer}$ et al, 2013). The combustion $\mathrm{CO}_{2}$ emissions $\mathrm{SO}_{\mathrm{X}}$ emissions during cruise tend to cool the climate, while $\mathrm{NO}_{\mathrm{X}}$ and $\mathrm{PM}$ emissions are generally predicted to warm the climate in terms of globally averaged surface temperature (RAND, 2009). $\mathrm{CO}_{2}$ emissions are released proportionally to the fuel combusted unlike $\mathrm{NO}_{\mathrm{x}}$ which is dependent on engine settings and flight altitude. More recent investigations have come to include PM and cloud-related phenomena such as linear contrails and aircraft-induced cirrus that affect the Earthatmosphere radiation balance. To add further complexity an aircrafts emission profile is also impacted by the interaction between the type of combustion technology and emissions location, and local atmospheric composition (Krammer et al, 2013). Depending on where within the upper atmosphere it is emitted, water vapor can be an important GHG (especially when emitted in the stratosphere, where approximately $20 \%$ to $40 \%$ of aircraft emissions are deposited). In contrast, water-vapor emissions at the ground and in the troposphere have a minor impact as a GHG because of the naturally large abundance of water in the hydrological cycle. This is a very interesting phenomenon as it implies if a fuel results in significant increases in water-vapor emissions when used in aviation, it might be advantageous from a global climate-change perspective to use that fuel in ground transportation (RAND, 2009).

As discussed in this study, presently the uncertainties associated with the treatment of fuel production and in-flight GHG emissions allow for a broad range of emission estimates and impacts for sustainable jetfuels. To better prepare commercial aviation for potential regulation of GHG emissions, a standard methodology for 
estimating lifecycle GHG-emission inventories and impacts is required. Such a methodology should include standards for accounting for key inputs, setting system boundaries, allocating emissions among alternative fuel products and co-products, all combustion emissions, and the effects of land-use change (RAND, 2009). As part of the certification process the US Federal Aviation Administration, US Air Force, NASA, and some international organizations are currently funding research to measure the emissions from burning alternative jetfuels. Continuing emission measurements are essential to assess accurately the impact of alternative-fuel combustion on both air quality and climate change (RAND, 2009).

In addition to meeting operational requirements and environmental commitments, it is critical for aviation to establish a universal and comprehensive methodology to evaluate the climate change impact of AJF for two important reasons. The first stems from the fact that in the next decade, large amounts of alcohol-based fuels and heavy fuels derived from oil sands and shale will likely enter the world oil market. The EIA estimates that heightened supply of unconventional crude will cut costs by $\$ 5$ to $\$ 13$ per barrel. A flood of cheaper fuels will make it even more difficult for advanced biofuels to compete on a price basis (RAND, 2009) unless policy measures are put in place that will set emission standards and penalties for surpassing them. Unconventional crude is abundant across many parts of the world but is also very expensive to extract and refine as well releases as much as $30 \%$ emissions per unit of energy compared to conventional crude. Applying a price on carbon will level the playing field and enforce a market-mediated transition to cleaner aviation fuels like AJF.

Secondly building consensus around a verifiable framework to quantify the non- $\mathrm{CO}_{2}$ climate change impact of aviation fuels will impart the ability for aviation to chart its actual performance along emissions standards and anticipate mitigation strategies accordingly even if some carbon pricing frameworks are implemented in the short term. Krammer et al (2013) show that in a scenario where EU ETS applied to aviation and maximum levels of biofuel is consumed, the long term $\mathrm{CO}_{2}$ and non- $\mathrm{CO}_{2}$ climate change impact start to diverge; $\mathrm{CO}_{2}$ impact levels off during 2020 to 2040, but non- $\mathrm{CO}_{2}$ impact continues to grow over this time period. The net result is that aviation's aggregate global warming potential continues to rise between 2020 and 2050 indicating the absence of a 'climate-neutral' growth period past 2020. In other words, unless advanced biofuels are designed to lower absolute emissions and their aggregate global warming potential, aviation's efforts at meeting both its growth projections and environmental standards will be very difficult once policy measures catch up to the most updated science (Krammer et al, 2013). 


\section{APPENDIX A}

\section{Baseline Calculations}

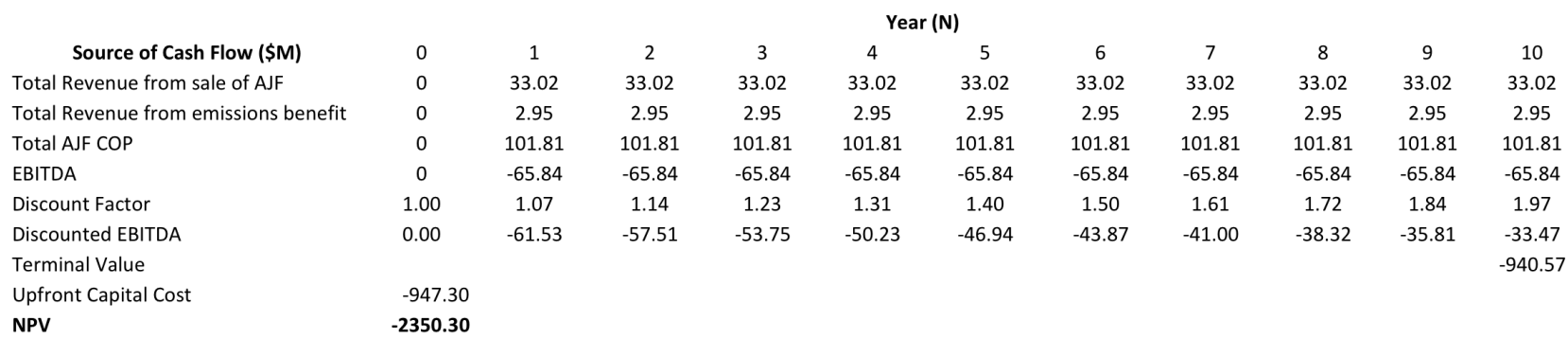

ASSUMPTIONS:

Algae oil content $=25 \%$

System productivity $=13.2 \mathrm{~g} / \mathrm{m} 2 /$ day

Total pond size $=4050$ ha

COP of AJF $=\$ 2.59 / \mathrm{L}$

Total production $=39.31 \mathrm{ML} / \mathrm{Y}$

Total COP per year $=(39,310,000 \mathrm{~L} / \mathrm{Y}) \times(\$ 2.59 / \mathrm{L})=\$ 101,812,900$

Total Revenue from Sale of AJF per year $=(39,310,000 \mathrm{~L} / \mathrm{Y}) \times(\$ 0.84 / \mathrm{L})=\$ 33,020,400$

Average price of Jetfuel for $2013=\$ 132.80 / \mathrm{bbl}$ or $\$$

Emissions price $=\$ 30 / \mathrm{tCO} 2 \mathrm{e}$

Energy Density of Bio-SPK $=43.5 \mathrm{MJ} / \mathrm{kg}$

Algal oil denisty $=920 \mathrm{~kg} / \mathrm{m} 3$

Total production per year by energy $(\mathrm{MJ})=(39,310,000 \mathrm{~L}) \times(920 \mathrm{~kg} / \mathrm{m3}) \times(\mathrm{m3} / 1000 \mathrm{I}) \times(43.5 \mathrm{MJ} / \mathrm{kg})=1,573,186,200 \mathrm{MJ}$

Life-Cylce Emissions intensity of AJF $=25 \mathrm{gCO} e / \mathrm{MJ}$

Life-Cycle Emissions intensity of PJF $=87.5 \mathrm{gCO} e / \mathrm{MJ}$

Total Life-Cycle Emission per year from AJF $=(1,573,186,200 \mathrm{MJ}) \times(25 \mathrm{gCO} e / \mathrm{MJ})=39,329,655,000 \mathrm{MJ}$

Total Life-Cycle Emission per year from PJF $=(1,573,186,200 \mathrm{MJ}) \times(87.5 \mathrm{gCO} 2 \mathrm{e} / \mathrm{MJ})=137,653,792,500 \mathrm{gCO} \mathrm{e}$

Total Life-Cycle Emission benefit per year $=(137,653,792,500)-(39,329,655,000)=98,324,137,500$ or $98,324.14$ tCO2

Total Monetized Life-Cycle Emission Benefit per year $=(98,324.14$ tCO2 $) \times(\$ 30 / \mathrm{tCO} 2)=\$ 2,949,724.20$

WACC $=7 \%$

$U C C=\$ 947.3 \mathrm{M}$ 


\section{APPENDIX B}

\section{Calculations for Changing JFP - @ \$1/1}

\begin{tabular}{|c|c|c|c|c|c|c|c|c|c|c|c|}
\hline & & & & & & $\operatorname{ear}(\mathrm{N})$ & & & & & \\
\hline Source of Cash Flow (\$M) & 0 & 1 & 2 & 3 & 4 & 5 & 6 & 7 & 8 & 9 & 10 \\
\hline Total Revenue from sale of AJF & 0 & 39.31 & 39.31 & 39.31 & 39.31 & 39.31 & 39.31 & 39.31 & 39.31 & 39.31 & 39.31 \\
\hline Total Revenue from emissions benefit & 0 & 2.95 & 2.95 & 2.95 & 2.95 & 2.95 & 2.95 & 2.95 & 2.95 & 2.95 & 2.95 \\
\hline Total AJF COP & 0 & 101.81 & 101.81 & 101.81 & 101.81 & 101.81 & 101.81 & 101.81 & 101.81 & 101.81 & 101.81 \\
\hline EBITDA & 0 & -59.55 & -59.55 & -59.55 & -59.55 & -59.55 & -59.55 & -59.55 & -59.55 & -59.55 & -59.55 \\
\hline Discount Factor & 1.00 & 1.07 & 1.14 & 1.23 & 1.31 & 1.40 & 1.50 & 1.61 & 1.72 & 1.84 & 1.97 \\
\hline Discounted EBITDA & 0.00 & -55.65 & -52.01 & -48.61 & -45.43 & -42.46 & -39.68 & -37.08 & -34.66 & -32.39 & -30.27 \\
\hline Terminal Value & & & & & & & & & & & -850.71 \\
\hline Upfront Capital Cost & -947.30 & & & & & & & & & & \\
\hline NPV & -2216.27 & & & & & & & & & & \\
\hline
\end{tabular}

\section{ASSUMPTIONS:}

Algae oil content $=25 \%$

System productivity $=13.2 \mathrm{~g} / \mathrm{m} 2 /$ day

Total pond size $=4050$ ha

COP of AJF $=\$ 2.59 / \mathrm{L}$

Total production $=39.31 \mathrm{ML} / \mathrm{Y}$

Total COP per year $=(39,310,000 \mathrm{~L} / \mathrm{Y}) \times(\$ 2.59 / \mathrm{L})=\$ 101,812,900$

Total Revenue from Sale of AJF per year $=(39,310,000 \mathrm{~L} / \mathrm{Y}) \times(\$ 1.00 / \mathrm{L})=\$ 39,310,000$

Hypothetical Jetfuel price $=\$ 1 / \mathrm{L}(\$ 158.76 / \mathrm{bbl})$

Emissions price $=\$ 30 / \mathrm{tCO} 2 \mathrm{e}$

Energy Density of Bio-SPK $=43.5 \mathrm{MJ} / \mathrm{kg}$

Algal oil denisty $=920 \mathrm{~kg} / \mathrm{m} 3$

Total production per year by energy $(\mathrm{MJ})=(39,310,000 \mathrm{~L}) \times(920 \mathrm{~kg} / \mathrm{m3}) \times(\mathrm{m} 3 / 1000 \mathrm{I}) \times(43.5 \mathrm{MJ} / \mathrm{kg})=1,573,186,200 \mathrm{M}$

Life-Cylce Emissions intensity of AJF $=25 \mathrm{gCO} e / \mathrm{MJ}$

Life-Cycle Emissions intensity of PJF $=87.5 \mathrm{gCO} 2 \mathrm{e} / \mathrm{MJ}$

Total Life-Cycle Emission per year from AJF $=(1,573,186,200 \mathrm{MJ}) \times(25 \mathrm{gCO} e / \mathrm{MJ})=39,329,655,000 \mathrm{MJ}$

Total Life-Cycle Emission per year from PJF $=(1,573,186,200 \mathrm{MJ}) \times(87.5 \mathrm{gCO} e / \mathrm{MJ})=137,653,792,500 \mathrm{gCO} e$

Total Life-Cycle Emission benefit per year $=(137,653,792,500)-(39,329,655,000)=98,324,137,500$ or $98,324.14$ tCO2

Total Monetized Life-Cycle Emission Benefit per year $=(98,324.14 \mathrm{tCO}) \times(\$ 30 / \mathrm{tCO})=\$ 2,949,724.20$

WACC $=7 \%$

Total UCC $=\$ 947.3 \mathrm{M}$ 


\section{Calculations for Changing JFP - @ 1.50/1}

\begin{tabular}{|c|c|c|c|c|c|c|c|c|c|c|c|}
\hline \multirow[b]{2}{*}{ Source of Cash Flow (\$M) } & \multicolumn{8}{|c|}{ Year (N) } & \multirow[b]{2}{*}{8} & \multirow[b]{2}{*}{9} & \multirow[b]{2}{*}{10} \\
\hline & 0 & 1 & 2 & 3 & 4 & 5 & 6 & 7 & & & \\
\hline Total Revenue from sale of AJF & 0 & 58.97 & 58.97 & 58.97 & 58.97 & 58.97 & 58.97 & 58.97 & 58.97 & 58.97 & 58.97 \\
\hline Total Revenue from emissions benefit & 0 & 2.95 & 2.95 & 2.95 & 2.95 & 2.95 & 2.95 & 2.95 & 2.95 & 2.95 & 2.95 \\
\hline Total AJF COP & 0 & 101.81 & 101.81 & 101.81 & 101.81 & 101.81 & 101.81 & 101.81 & 101.81 & 101.81 & 101.81 \\
\hline EBITDA & 0 & -39.89 & -39.89 & -39.89 & -39.89 & -39.89 & -39.89 & -39.89 & -39.89 & -39.89 & -39.89 \\
\hline Discount Factor & 1.00 & 1.07 & 1.14 & 1.23 & 1.31 & 1.40 & 1.50 & 1.61 & 1.72 & 1.84 & 1.97 \\
\hline Discounted EBITDA & 0.00 & -37.28 & -34.84 & -32.56 & -30.43 & -28.44 & -26.58 & -24.84 & -23.22 & -21.70 & -20.28 \\
\hline Terminal Value & & & & & & & & & & & -569.86 \\
\hline Upfront Capital Cost & -947.30 & & & & & & & & & & \\
\hline NPV & -1797.33 & & & & & & & & & & \\
\hline \multicolumn{12}{|l|}{ ASSUMPTIONS: } \\
\hline \multicolumn{12}{|l|}{ Algae oil content $=25 \%$} \\
\hline \multicolumn{12}{|l|}{ System productivity $=13.2 \mathrm{~g} / \mathrm{m} 2 /$ day } \\
\hline \multicolumn{12}{|l|}{ Total pond size $=4050$ ha } \\
\hline \multicolumn{12}{|l|}{ COP of AJF $=\$ 2.59 / \mathrm{L}$} \\
\hline \multicolumn{12}{|l|}{ Total production $=39.31 \mathrm{ML} / \mathrm{Y}$} \\
\hline \multicolumn{12}{|c|}{ Total COP per year $=(39,310,000 \mathrm{~L} / \mathrm{Y}) \times(\$ 2.59 / \mathrm{L})=\$ 101,812,900$} \\
\hline \multicolumn{12}{|c|}{ Total Revenue from Sale of AJF per year $=(39,310,000 \mathrm{~L} / \mathrm{Y}) \times(\$ 1.50 / \mathrm{L})=\$ 58,965,000$} \\
\hline \multicolumn{12}{|c|}{ Hypothetical Jetfuel price $=\$ 1.50 / \mathrm{L}(\$ 238.76 / \mathrm{bbl})$} \\
\hline \multicolumn{12}{|l|}{ Emissions price $=\$ 30 / \mathrm{tCO} 2 \mathrm{e}$} \\
\hline \multicolumn{12}{|l|}{ Energy Density of Bio-SPK $=43.5 \mathrm{MJ} / \mathrm{kg}$} \\
\hline \multicolumn{12}{|l|}{ Algal oil denisty $=920 \mathrm{~kg} / \mathrm{m} 3$} \\
\hline \multicolumn{12}{|c|}{ Total production per year by energy $(\mathrm{MJ})=(39,310,000 \mathrm{~L}) \times(920 \mathrm{~kg} / \mathrm{m3}) \times(\mathrm{m} 3 / 1000 \mathrm{I}) \times(43.5 \mathrm{MJ} / \mathrm{kg})=1,573,186,200 \mathrm{MJ}$} \\
\hline \multicolumn{12}{|c|}{ Life-Cylce Emissions intensity of AJF = $25 \mathrm{gCO} 2 \mathrm{e} / \mathrm{MJ}$} \\
\hline \multicolumn{12}{|c|}{ Life-Cycle Emissions intensity of PJF $=87.5 \mathrm{gCO} 2 \mathrm{e} / \mathrm{MJ}$} \\
\hline \multicolumn{12}{|c|}{ Total Life-Cycle Emission per year from AJF $=(1,573,186,200 \mathrm{MJ}) \times(25 \mathrm{gCO} e / \mathrm{MJ})=39,329,655,000 \mathrm{MJ}$} \\
\hline \multicolumn{12}{|c|}{ Total Life-Cycle Emission per year from PJF = $(1,573,186,200 \mathrm{MJ}) \times(87.5 \mathrm{gCO} 2 \mathrm{e} / \mathrm{MJ})=137,653,792,500 \mathrm{gCO} \mathrm{e}$} \\
\hline \multicolumn{12}{|c|}{ Total Life-Cycle Emission benefit per year $=(137,653,792,500)-(39,329,655,000)=98,324,137,500$ or $98,324.14$ tCO2 } \\
\hline \multicolumn{12}{|c|}{ Total Monetized Life-Cycle Emission Benefit per year $=(98,324.14 \mathrm{tCO} 2) \times(\$ 30 / \mathrm{tCO} 2)=\$ 2,949,724.20$} \\
\hline \multicolumn{12}{|l|}{ WACC $=7 \%$} \\
\hline Total UCC $=\$ 947.3 \mathrm{M}$ & & & & & & & & & & & \\
\hline
\end{tabular}




\section{Calculations for Changing JFP - @ \$3/1}

\begin{tabular}{|c|c|c|c|c|c|c|c|c|c|c|c|}
\hline \multirow[b]{2}{*}{ Source of Cash Flow (\$M) } & \multicolumn{9}{|c|}{ Year (N) } & \multirow[b]{2}{*}{9} & \multirow[b]{2}{*}{10} \\
\hline & 0 & 1 & 2 & 3 & 4 & 5 & 6 & 7 & 8 & & \\
\hline Total Revenue from sale of AJF & 0 & 117.93 & 117.93 & 117.93 & 117.93 & 117.93 & 117.93 & 117.93 & 117.93 & 117.93 & 117.93 \\
\hline Total Revenue from emissions benefit & 0 & 2.95 & 2.95 & 2.95 & 2.95 & 2.95 & 2.95 & 2.95 & 2.95 & 2.95 & 2.95 \\
\hline Total AJF COP & 0 & 101.81 & 101.81 & 101.81 & 101.81 & 101.81 & 101.81 & 101.81 & 101.81 & 101.81 & 101.81 \\
\hline EBITDA & 0 & 19.07 & 19.07 & 19.07 & 19.07 & 19.07 & 19.07 & 19.07 & 19.07 & 19.07 & 19.07 \\
\hline Discount Factor & 1.00 & 1.07 & 1.14 & 1.23 & 1.31 & 1.40 & 1.50 & 1.61 & 1.72 & 1.84 & 1.97 \\
\hline Discounted EBITDA & 0.00 & 17.82 & 16.66 & 15.57 & 14.55 & 13.60 & 12.71 & 11.88 & 11.10 & 10.37 & 9.69 \\
\hline Terminal Value & & & & & & & & & & & 272.43 \\
\hline Upfront Capital Cost & -947 & & & & & & & & & & \\
\hline
\end{tabular}

$-540.93$

ASSUMPTIONS:

Algae oil content $=25 \%$

System productivity $=13.2 \mathrm{~g} / \mathrm{m} 2 /$ day

Total pond size $=4050$ ha

COP of AJF $=\$ 2.59 / \mathrm{L}$

Total production $=39.31 \mathrm{ML} / \mathrm{Y}$

Total COP per year $=(39,310,000 \mathrm{~L} / \mathrm{Y}) \times(\$ 2.59 / \mathrm{L})=\$ 101,812,900$

Total Revenue from Sale of AJF per year $=(39,310,000 \mathrm{~L} / \mathrm{Y}) \times(\$ 3.00 / \mathrm{L})=\$ 117,930,000$

Hypothetical Jetfuel price $=\$ 3 / \mathrm{L}(\$ 476.28 / \mathrm{bb})$

Emissions price $=\$ 30 / \mathrm{tCO} 2$

Energy Density of Bio-SPK $=43.5 \mathrm{MJ} / \mathrm{kg}$

Algal oil denisty $=920 \mathrm{~kg} / \mathrm{m} 3$

Total production per year by energy $(\mathrm{MJ})=(39,310,000 \mathrm{~L}) \times(920 \mathrm{~kg} / \mathrm{m3}) \times(\mathrm{m3} / 1000 \mathrm{I}) \times(43.5 \mathrm{MJ} / \mathrm{kg})=1,573,186,200 \mathrm{MJ}$

Life-Cylce Emissions intensity of AJF $=25 \mathrm{gCO} e / \mathrm{MJ}$

Life-Cycle Emissions intensity of PJF $=87.5 \mathrm{gCO} 2 \mathrm{e} / \mathrm{MJ}$

Total Life-Cycle Emission per year from AJF $=(1,573,186,200 \mathrm{MJ}) \times(25 \mathrm{gCO} e / \mathrm{MJ})=39,329,655,000 \mathrm{M}$

Total Life-Cycle Emission per year from PJF $=(1,573,186,200 \mathrm{MJ}) \times(87.5 \mathrm{gCO} 2 \mathrm{e} / \mathrm{MJ})=137,653,792,500 \mathrm{gCO} \mathrm{e}$

Total Life-Cycle Emission benefit per year $=(137,653,792,500)-(39,329,655,000)=98,324,137,500$ or $98,324.14$ tCO2

Total Monetized Life-Cycle Emission Benefit per year $=(98,324.14 \mathrm{tCO} 2) \times(\$ 30 / \mathrm{tCO} 2)=\$ 2,949,724.20$

WACC $=7 \%$

Total UCC $=\$ 947.3 \mathrm{M}$ 


\section{Calculations for Changing JFP - @ \$6/1}

\begin{tabular}{|c|c|c|c|c|c|c|c|c|c|c|c|}
\hline \multirow[b]{2}{*}{ Source of Cash Flow (\$M) } & \multicolumn{8}{|c|}{ Year (N) } & \multirow[b]{2}{*}{8} & \multirow[b]{2}{*}{9} & \multirow[b]{2}{*}{10} \\
\hline & 0 & 1 & 2 & 3 & 4 & 5 & 6 & 7 & & & \\
\hline Total Revenue from sale of AJF & 0 & 235.86 & 235.86 & 235.86 & 235.86 & 235.86 & 235.86 & 235.86 & 235.86 & 235.86 & 235.86 \\
\hline Total Revenue from emissions benefit & 0 & 2.95 & 2.95 & 2.95 & 2.95 & 2.95 & 2.95 & 2.95 & 2.95 & 2.95 & 2.95 \\
\hline Total AJF COP & 0 & 101.81 & 101.81 & 101.81 & 101.81 & 101.81 & 101.81 & 101.81 & 101.81 & 101.81 & 101.81 \\
\hline EBITDA & 0 & 137.00 & 137.00 & 137.00 & 137.00 & 137.00 & 137.00 & 137.00 & 137.00 & 137.00 & 137.00 \\
\hline Discount Factor & 1.00 & 1.07 & 1.14 & 1.23 & 1.31 & 1.40 & 1.50 & 1.61 & 1.72 & 1.84 & 1.97 \\
\hline Discounted EBITDA & 0.00 & 128.04 & 119.66 & 111.83 & 104.52 & 97.68 & 91.29 & 85.32 & 79.74 & 74.52 & 69.64 \\
\hline Terminal Value & & & & & & & & & & & 1957.14 \\
\hline Upfront Capital Cost & -947.30 & & & & & & & & & & \\
\hline NPV & 2919.37 & & & & & & & & & & \\
\hline
\end{tabular}

\section{ASSUMPTIONS:}

Algae oil content $=25 \%$

System productivity $=13.2 \mathrm{~g} / \mathrm{m} 2 /$ day

Total pond size $=4050$ ha

COP of AJF $=\$ 2.59 / \mathrm{L}$

Total production $=39.31 \mathrm{ML} / \mathrm{Y}$

Total COP per year $=(39,310,000 \mathrm{~L} / \mathrm{Y}) \times(\$ 2.59 / \mathrm{L})=\$ 101,812,900$

Total Revenue from Sale of Algal-Jet per year $=(39,310,000 \mathrm{~L} / \mathrm{Y}) \times(\$ 6.00 / \mathrm{L})=\$ 235,860,000$

Hypothetical Jetfuel price $=\$ 6 / \mathrm{L}(\$ 952.56 / \mathrm{bbl})$

Emissions price $=\$ 30 / \mathrm{tCO} 2 \mathrm{e}$

Energy Density of Bio-SPK $=43.5 \mathrm{MJ} / \mathrm{kg}$

Algal oil denisty $=920 \mathrm{~kg} / \mathrm{m} 3$

Total production per year by energy $(\mathrm{MJ})=(39,310,000 \mathrm{~L}) \times(920 \mathrm{~kg} / \mathrm{m3}) \times(\mathrm{m3} / 1000 \mathrm{I}) \times(43.5 \mathrm{MJ} / \mathrm{kg})=1,573,186,200 \mathrm{MJ}$

Life-Cylce Emissions intensity of AJF $=25 \mathrm{gCO} e / \mathrm{MJ}$

Life-Cycle Emissions intensity of PJF $=87.5 \mathrm{gCO} e / \mathrm{MJ}$

Total Life-Cycle Emission per year from AJF $=(1,573,186,200 \mathrm{MJ}) \times(25 \mathrm{gCO} \mathrm{e} / \mathrm{MJ})=39,329,655,000 \mathrm{MJ}$

Total Life-Cycle Emission per year from PJF $=(1,573,186,200 \mathrm{MJ}) \times(87.5 \mathrm{gCO} 2 \mathrm{e} / \mathrm{MJ})=137,653,792,500 \mathrm{gCO} 2 \mathrm{e}$

Total Life-Cycle Emission benefit per year $=(137,653,792,500)-(39,329,655,000)=98,324,137,500$ or 98,324.14 tCO2

Total Monetized Life-Cycle Emission Benefit per year $=(98,324.14 \mathrm{tCO}) \times(\$ 30 / \mathrm{tCO} 2)=\$ 2,949,724.20$

WACC $=7 \%$

Total UCC $=\$ 947.3 \mathrm{M}$ 


\section{APPENDIX C}

\section{Calculations for Carbon Tax @ \$60/tCO 2 e}

\begin{tabular}{|c|c|c|c|c|c|c|c|c|c|c|c|}
\hline \multirow[b]{2}{*}{ Source of Cash Flow (\$M) } & \multicolumn{9}{|c|}{ Year (N) } & \multirow[b]{2}{*}{9} & \multirow[b]{2}{*}{10} \\
\hline & 0 & 1 & 2 & 3 & 4 & 5 & 6 & 7 & 8 & & \\
\hline Total Revenue from sale of AJF & 0 & 33.02 & 33.02 & 33.02 & 33.02 & 33.02 & 33.02 & 33.02 & 33.02 & 33.02 & 33.02 \\
\hline Total Revenue from emissions benefit & 0 & 5.90 & 5.90 & 5.90 & 5.90 & 5.90 & 5.90 & 5.90 & 5.90 & 5.90 & 5.90 \\
\hline Total COP of AJF & 0 & 101.8 & 101.81 & 101.81 & 101.81 & 101.81 & 101.81 & 101.81 & 101.81 & 101.81 & 101.81 \\
\hline EBITDA & 0 & -62.89 & -62.89 & -62.89 & -62.89 & -62.89 & -62.89 & -62.89 & -62.89 & -62.89 & -62.89 \\
\hline Discount Factor & 1.00 & 1.07 & 1.14 & 1.23 & 1.31 & 1.40 & 1.50 & 1.61 & 1.72 & 1.84 & 1.97 \\
\hline Discounted EBITDA & 0.00 & -58.78 & -54.93 & -51.34 & -47.98 & -44.84 & -41.91 & -39.16 & -36.60 & -34.21 & -31.97 \\
\hline Terminal Value & & & & & & & & & & & -898.43 \\
\hline Upfront Capital Cost & -947.3 & & & & & & & & & & \\
\hline NPV & -2287.44 & & & & & & & & & & \\
\hline
\end{tabular}

\section{ASSUMPTIONS:}

Algae oil content $=25 \%$

System productivity $=13.2 \mathrm{~g} / \mathrm{m} 2 /$ day

Total pond size $=4050$ ha

COP of AJF $=\$ 2.59 / \mathrm{L}$

Total production $=39.31 \mathrm{ML} / \mathrm{Y}$

Total COP per year $=(39,310,000 \mathrm{~L} / \mathrm{Y}) \times(\$ 2.59 / \mathrm{L})=\$ 101,812,900$

Total Revenue from Sale of AJF per year $=(39,310,000 \mathrm{~L} / \mathrm{Y}) \times(\$ 0.84 / \mathrm{L})=\$ 33,020,400$

Average price of Jetfuel for $2013=\$ 132.80 / \mathrm{bbl}$ or $\$ 0.84 / \mathrm{L}$

Emissions price $=\$ 60 / \mathrm{tCO} 2 \mathrm{e}$

Energy Density of Bio-SPK $=43.5 \mathrm{MJ} / \mathrm{kg}$

Algal oil denisty $=920 \mathrm{~kg} / \mathrm{m} 3$

Total production per year by energy $(\mathrm{MJ})=(39,310,000 \mathrm{~L}) \times(920 \mathrm{~kg} / \mathrm{m3}) \times(\mathrm{m3} / 1000 \mathrm{I}) \times(43.5 \mathrm{MJ} / \mathrm{kg})=1,573,186,200 \mathrm{MJ}$

Life-Cylce Emissions intensity of AJF $=25 \mathrm{gCO} 2 \mathrm{e} / \mathrm{MJ}$

Life-Cycle Emissions intensity of PJF $=87.5 \mathrm{gCO} e / \mathrm{MJ}$

Total Life-Cycle Emission per year from AJF $=(1,573,186,200 \mathrm{MJ}) \times(25 \mathrm{gCO} \mathrm{e} / \mathrm{MJ})=39,329,655,000 \mathrm{MJ}$

Total Life-Cycle Emission per year from PJF $=(1,573,186,200 \mathrm{MJ}) \times(87.5 \mathrm{gCO} e / \mathrm{MJ})=137,653,792,500 \mathrm{gCO} 2 \mathrm{e}$

Total Life-Cycle Emission benefit per year $=(137,653,792,500)-(39,329,655,000)=98,324,137,500$ or $98,324.14$ tCO2

Total Monetized Life-Cycle Emission Benefit per year $=(98,324.14 \mathrm{tCO} 2) \times(\$ 60 / \mathrm{tCO})=\$ 5,899,448.25$

WACC $=7 \%$

Total UCC $=\$ 947.3 \mathrm{M}$ 


\section{Calculations for Carbon Tax @ \$120/tCO $2 \mathrm{e}$}

\begin{tabular}{|c|c|c|c|c|c|c|c|c|c|c|c|}
\hline \multirow[b]{2}{*}{ Source of Cash Flow (\$M) } & \multicolumn{10}{|c|}{ Year (N) } & \multirow[b]{2}{*}{10} \\
\hline & $\mathbf{0}$ & 1 & 2 & 3 & 4 & 5 & 6 & 7 & 8 & 9 & \\
\hline Total Revenue from sale of AJF & 0 & 33.02 & 33.02 & 33.02 & 33.02 & 33.02 & 33.02 & 33.02 & 33.02 & 33.02 & 33.02 \\
\hline Total Revenue from emissions benefit & 0 & 11.98 & 11.98 & 11.98 & 11.98 & 11.98 & 11.98 & 11.98 & 11.98 & 11.98 & 11.98 \\
\hline Total COP of AJF & 0 & 101.8 & 101.81 & 101.81 & 101.81 & 101.81 & 101.81 & 101.81 & 101.81 & 101.81 & 101.81 \\
\hline EBITDA & 0 & -56.81 & -56.81 & -56.81 & -56.81 & -56.81 & -56.81 & -56.81 & -56.81 & -56.81 & -56.81 \\
\hline Discount Factor & 1.00 & 1.07 & 1.14 & 1.23 & 1.31 & 1.40 & 1.50 & 1.61 & 1.72 & 1.84 & 1.97 \\
\hline Discounted EBITDA & 0.00 & -53.09 & -49.62 & -46.37 & -43.34 & -40.50 & -37.85 & -35.38 & -33.06 & -30.90 & -28.88 \\
\hline Terminal Value & & & & & & & & & & & -811.57 \\
\hline Upfront Capital Cost & -947.30 & & & & & & & & & & \\
\hline NPV & -2157.88 & & & & & & & & & & \\
\hline
\end{tabular}

ASSUMPTIONS:

Algae oil content $=25 \%$

System productivity $=13.2 \mathrm{~g} / \mathrm{m} 2 /$ day

Total pond size $=4050 \mathrm{ha}$

COP of AJF $=\$ 2.59 / \mathrm{L}$

Total production $=39.31 \mathrm{ML} / \mathrm{Y}$

Total COP per year $=(39,310,000 \mathrm{~L} / \mathrm{Y}) \times(\$ 2.59 / \mathrm{L})=\$ 101,812,900$

Total Revenue from Sale of AJF per year $=(39,310,000 \mathrm{~L} / \mathrm{Y}) \times(\$ 0.84 / \mathrm{L})=\$ 33,020,400$

Average price of Jetfuel for $2013=\$ 132.80 / \mathrm{bbl}$ or $\$ 0.84 / \mathrm{L}$

Emissions price $=\$ 120 / \mathrm{tCO} 2 \mathrm{e}$

Energy Density of Bio-SPK $=43.5 \mathrm{MJ} / \mathrm{kg}$

Algal oil denisty $=920 \mathrm{~kg} / \mathrm{m} 3$

Total production per year by energy $(\mathrm{MJ})=(39,310,000 \mathrm{~L}) \times(920 \mathrm{~kg} / \mathrm{m} 3) \times(\mathrm{m} 3 / 1000 \mathrm{I}) \times(43.5 \mathrm{MJ} / \mathrm{kg})=1,573,186,200 \mathrm{MJ}$

Life-Cylce Emissions intensity of AJF $=25 \mathrm{gCO} e / \mathrm{MJ}$

Life-Cycle Emissions intensity of PJF $=87.5 \mathrm{gCO} e / \mathrm{MJ}$

Total Life-Cycle Emission per year from AJF $=(1,573,186,200 \mathrm{MJ}) \times(25 \mathrm{gCO} 2 \mathrm{e} / \mathrm{MJ})=39,329,655,000 \mathrm{MJ}$

Total Life-Cycle Emission per year from PJF $=(1,573,186,200 \mathrm{MJ}) \times(87.5 \mathrm{gCO} \mathrm{e} / \mathrm{MJ})=137,653,792,500 \mathrm{gCO} 2 \mathrm{e}$

Total Life-Cycle Emission benefit per year $=(137,653,792,500)-(39,329,655,000)=98,324,137,500$ or $98,324.14$ tCO2

Total Monetized Life-Cycle Emission Benefit per year $=(98,324.14 \mathrm{tCO} 2) \times(\$ 120 / \mathrm{tCO})=\$ 11,978,896.50$

WACC $=7 \%$

Total UCC $=\$ 947.3 \mathrm{M}$ 


\section{Calculations for Carbon Tax @ \$200/tC02e}

$\begin{array}{lccccccccccc}\quad \text { Source of Cash Flow (\$M) } & \mathbf{0} & \mathbf{1} & \mathbf{2} & \mathbf{3} & \mathbf{4} & \mathbf{5} & \mathbf{6} & \mathbf{7} & \mathbf{8} & \mathbf{9} & \mathbf{1 0} \\ \text { Total Revenue from sale of AJF } & 0 & 33.02 & 33.02 & 33.02 & 33.02 & 33.02 & 33.02 & 33.02 & 33.02 & 33.02 & 33.02 \\ \text { Total Revenue from emissions benefit } & 0 & 19.66 & 19.66 & 19.66 & 19.66 & 19.66 & 19.66 & 19.66 & 19.66 & 19.66 & 19.66 \\ \text { Total COP of AJF } & 0 & 101.8 & 101.81 & 101.81 & 101.81 & 101.81 & 101.81 & 101.81 & 101.81 & 101.81 & 101.81 \\ \text { EBITDA } & 0 & -49.13 & -49.13 & -49.13 & -49.13 & -49.13 & -49.13 & -49.13 & -49.13 & -49.13 & -49.13 \\ \text { Discount Factor } & 1.00 & 1.07 & 1.14 & 1.23 & 1.31 & 1.40 & 1.50 & 1.61 & 1.72 & 1.84 & 1.97 \\ \text { Discounted EBITDA } & 0.00 & -45.92 & -42.91 & -40.10 & -37.48 & -35.03 & -32.74 & -30.60 & -28.59 & -26.72 & -24.98 \\ \text { Terminal Value } & & & & & & & & & & \end{array}$

\section{ASSUMPTIONS:}

Algae oil content $=25 \%$

System productivity $=13.2 \mathrm{~g} / \mathrm{m} 2 /$ day

Total pond size $=4050$ ha

COP of AJF $=\$ 2.59 / \mathrm{L}$

Total production $=39.31 \mathrm{ML} / \mathrm{Y}$

Total COP per year $=(39,310,000 \mathrm{~L} / \mathrm{Y}) \times(\$ 2.59 / \mathrm{L})=\$ 101,812,900$

Total Revenue from Sale of AJF per year $=(39,310,000 \mathrm{~L} / \mathrm{Y}) \times(\$ 0.84 / \mathrm{L})=\$ 33,020,400$

Average price of Jetfuel for $2013=\$ 132.80 / \mathrm{bbl}$ or $\$ 0.84 / \mathrm{L}$

Emissions price $=\$ 200 / \mathrm{tCO} 2 \mathrm{e}$

Energy Density of Bio-SPK $=43.5 \mathrm{MJ} / \mathrm{kg}$

Algal oil denisty $=920 \mathrm{~kg} / \mathrm{m} 3$

Total production per year by energy $(\mathrm{MJ})=(39,310,000 \mathrm{~L}) \times(920 \mathrm{~kg} / \mathrm{m3}) \times(\mathrm{m} 3 / 1000 \mathrm{I}) \times(43.5 \mathrm{MJ} / \mathrm{kg})=1,573,186,200 \mathrm{MJ}$

Life-Cylce Emissions intensity of AJF $=25 \mathrm{gCO} e / \mathrm{MJ}$

Life-Cycle Emissions intensity of PJF $=87.5 \mathrm{gCO} 2 \mathrm{e} / \mathrm{MJ}$

Total Life-Cycle Emission per year from AJF $=(1,573,186,200 \mathrm{MJ}) \times(25 \mathrm{gCO} e / \mathrm{MJ})=39,329,655,000 \mathrm{MJ}$

Total Life-Cycle Emission per year from PJF $=(1,573,186,200 \mathrm{MJ}) \times(87.5 \mathrm{gCO} \mathrm{e} / \mathrm{MJ})=137,653,792,500 \mathrm{gCO} \mathrm{e}$

Total Life-Cycle Emission benefit per year $=(137,653,792,500)-(39,329,655,000)=98,324,137,500$ or $98,324.14$ tCO2

Total Monetized Life-Cycle Emission Benefit per year $=(98,324.14 \mathrm{tCO} 2) \times(\$ 200 / \mathrm{tCO} 2)=\$ 19,664,827.50$

WACC $=7 \%$

Total UCC $=\$ 947.3 \mathrm{M}$ 


\section{Calculations for Carbon Tax @ \$400/tCO2e}

\begin{tabular}{|c|c|c|c|c|c|c|c|c|c|c|c|}
\hline \multirow[b]{2}{*}{ Source of Cash Flow (\$M) } & \multicolumn{9}{|c|}{ Year (N) } & \multirow[b]{2}{*}{9} & \multirow[b]{2}{*}{10} \\
\hline & $\mathbf{0}$ & 1 & 2 & 3 & 4 & 5 & 6 & 7 & 8 & & \\
\hline Total Revenue from emissions benefit & 0 & 39.33 & 39.33 & 39.33 & 39.33 & 39.33 & 39.33 & 39.33 & 39.33 & 39.33 & 39.33 \\
\hline EBITDA & 0 & -29.46 & -29.46 & -29.46 & -29.46 & -29.46 & -29.46 & -29.46 & -29.46 & -29.46 & -29.46 \\
\hline Discount Factor & 1.00 & 1.07 & 1.14 & 1.23 & 1.31 & 1.40 & 1.50 & 1.61 & 1.72 & 1.84 & 1.97 \\
\hline Discounted EBITDA & 0.00 & -27.53 & -25.73 & -24.05 & -22.47 & -21.00 & -19.63 & -18.35 & -17.15 & -16.02 & -14.98 \\
\hline NPV & -1575.07 & & & & & & & & & & \\
\hline
\end{tabular}

\section{ASSUMPTIONS:}

Algae oil content $=25 \%$

System productivity $=13.2 \mathrm{~g} / \mathrm{m} 2 /$ day

Total pond size $=4050$ ha

COP of AJF $=\$ 2.59 / \mathrm{L}$

Total production $=39.31 \mathrm{ML} / \mathrm{Y}$

Total COP per year $=(39,310,000 \mathrm{~L} / \mathrm{Y}) \times(\$ 2.59 / \mathrm{L})=\$ 101,812,900$

Total Revenue from Sale of AJF per year $=(39,310,000 \mathrm{~L} / \mathrm{Y}) \times(\$ 0.84 / \mathrm{L})=\$ 33,020,400$

Average price of Jetfuel for $2013=\$ 132.80 / \mathrm{bbl}$ or $\$ 0.84 / \mathrm{L}$

Emissions price $=\$ 400 / \mathrm{tCO} 2 \mathrm{e}$

Energy Density of Bio-SPK $=43.5 \mathrm{MJ} / \mathrm{kg}$

Algal oil denisty $=920 \mathrm{~kg} / \mathrm{m} 3$

Total production per year by energy $(\mathrm{MJ})=(39,310,000 \mathrm{~L}) \times(920 \mathrm{~kg} / \mathrm{m3}) \times(\mathrm{m} 3 / 1000 \mathrm{l}) \times(43.5 \mathrm{MJ} / \mathrm{kg})=1,573,186,200 \mathrm{MJ}$

Life-Cylce Emissions intensity of AJF $=25 \mathrm{gCO} 2 \mathrm{e} / \mathrm{MJ}$

Life-Cycle Emissions intensity of PJF $=87.5 \mathrm{gCO} e / \mathrm{MJ}$

Total Life-Cycle Emission per year from AJF $=(1,573,186,200 \mathrm{MJ}) \times(25 \mathrm{gCO} e / \mathrm{MJ})=39,329,655,000 \mathrm{MJ}$

Total Life-Cycle Emission per year from PJF $=(1,573,186,200 \mathrm{MJ}) \times(87.5 \mathrm{gCO} 2 \mathrm{e} / \mathrm{MJ})=137,653,792,500 \mathrm{gCO} \mathrm{e}$

Total Life-Cycle Emission benefit per year $=(137,653,792,500)-(39,329,655,000)=98,324,137,500$ or $98,324.14$ tCO2

Total Monetized Life-Cycle Emission Benefit per year $=(98,324.14 \mathrm{tCO}) \times(\$ 400 / \mathrm{tCO} 2)=\$ 39,329,656$

$\mathrm{WACC}=7 \%$

Total UCC $=\$ 947.3 \mathrm{M}$ 


\section{Calculations for Carbon Tax @ \$800/tCO2e}

\begin{tabular}{lccccccccccc}
\multicolumn{1}{r}{ Source of Cash Flow (\$M) } & $\mathbf{0}$ & $\mathbf{1}$ & $\mathbf{2}$ & $\mathbf{3}$ & $\mathbf{4}$ & $\mathbf{5}$ & $\mathbf{6}$ & $\mathbf{7}$ & $\mathbf{8}$ & $\mathbf{9}$ & $\mathbf{1 0}$ \\
Total Revenue from sale of AJF & 0 & 33.02 & 33.02 & 33.02 & 33.02 & 33.02 & 33.02 & 33.02 & 33.02 & 33.02 & 33.02 \\
Total Revenue from emissions benefit & 0 & 78.66 & 78.66 & 78.66 & 78.66 & 78.66 & 78.66 & 78.66 & 78.66 & 78.66 & 78.66 \\
Total COP of AJF & 0 & 101.8 & 101.81 & 101.81 & 101.81 & 101.81 & 101.81 & 101.81 & 101.81 & 101.81 & 101.81 \\
EBITDA & 0 & 9.87 & 9.87 & 9.87 & 9.87 & 9.87 & 9.87 & 9.87 & 9.87 & 9.87 & 9.87 \\
Discount Factor & 1.00 & 1.07 & 1.14 & 1.23 & 1.31 & 1.40 & 1.50 & 1.61 & 1.72 & 1.84 & 1.97 \\
Discounted EBITDA & 0.00 & 9.22 & 8.62 & 8.06 & 7.53 & 7.04 & 6.58 & 6.15 & 5.74 & 5.37 & 5.02 \\
Terminal Value & & & & & & & & & &
\end{tabular}

\section{ASSUMPTIONS:}

Algae oil content $=25 \%$

System productivity $=13.2 \mathrm{~g} / \mathrm{m} 2 /$ day

Total pond size $=4050$ ha

COP of AJF $=\$ 2.59 / \mathrm{L}$

Total production $=39.31 \mathrm{ML} / \mathrm{Y}$

Total COP per year $=(39,310,000 \mathrm{~L} / \mathrm{Y}) \times(\$ 2.59 / \mathrm{L})=\$ 101,812,900$

Total Revenue from Sale of AJF per year $=(39,310,000 \mathrm{~L} / \mathrm{Y}) \times(\$ 0.84 / \mathrm{L})=\$ 33,020,400$

Average price of Jetfuel for $2013=\$ 132.80 / \mathrm{bbl}$ or $\$ 0.84 / \mathrm{L}$

Emissions price $=\$ 800 / \mathrm{tCO} 2 \mathrm{e}$

Energy Density of Bio-SPK $=43.5 \mathrm{MJ} / \mathrm{kg}$

Algal oil denisty $=920 \mathrm{~kg} / \mathrm{m} 3$

Total production per year by energy $(\mathrm{MJ})=(39,310,000 \mathrm{~L}) \times(920 \mathrm{~kg} / \mathrm{m3}) \times(\mathrm{m} 3 / 1000 \mathrm{I}) \times(43.5 \mathrm{MJ} / \mathrm{kg})=1,573,186,200 \mathrm{MJ}$

Life-Cylce Emissions intensity of AJF $=25 \mathrm{gCO} e / \mathrm{MJ}$

Life-Cycle Emissions intensity of PJF $=87.5 \mathrm{gCO} e / \mathrm{MJ}$

Total Life-Cycle Emission per year from AJF $=(1,573,186,200 \mathrm{MJ}) \times(25 \mathrm{gCO} e / \mathrm{MJ})=39,329,655,000 \mathrm{MJ}$

Total Life-Cycle Emission per year from PJF $=(1,573,186,200 \mathrm{MJ}) \times(87.5 \mathrm{gCO} e / \mathrm{MJ})=137,653,792,500 \mathrm{gCO} e$

Total Life-Cycle Emission benefit per year $=(137,653,792,500)-(39,329,655,000)=98,324,137,500$ or $98,324.14$ tCO2

Total Monetized Life-Cycle Emission Benefit per year $=(98,324.14$ tCO2 $) \times(\$ 800 / \mathrm{tCO})=\$ 78,659,312$

WACC $=7 \%$

Total UCC $=\$ 947.3 \mathrm{M}$ 


\section{Calculations for Carbon Tax @ \$2000/tCO2e}

\begin{tabular}{lccccccccccc}
\multicolumn{1}{r}{$\quad$ Source of Cash Flow (\$M) } & $\mathbf{0}$ & $\mathbf{1}$ & $\mathbf{2}$ & $\mathbf{3}$ & $\mathbf{4}$ & $\mathbf{5}$ & $\mathbf{6}$ & $\mathbf{7}$ & $\mathbf{8}$ & $\mathbf{9}$ & $\mathbf{1 0}$ \\
Total Revenue from sale of AJF & 0 & 33.02 & 33.02 & 33.02 & 33.02 & 33.02 & 33.02 & 33.02 & 33.02 & 33.02 & 33.02 \\
Total Revenue from emissions benefit & 0 & 196.65 & 196.65 & 196.65 & 196.65 & 196.65 & 196.65 & 196.65 & 196.65 & 196.65 & 196.65 \\
Total COP of AJF & 0 & 101.8 & 101.81 & 101.81 & 101.81 & 101.81 & 101.81 & 101.81 & 101.81 & 101.81 & 101.81 \\
EBITDA & 0 & 127.86 & 127.86 & 127.86 & 127.86 & 127.86 & 127.86 & 127.86 & 127.86 & 127.86 & 127.86 \\
Discount Factor & 1.00 & 1.07 & 1.14 & 1.23 & 1.31 & 1.40 & 1.50 & 1.61 & 1.72 & 1.84 & 1.97 \\
Discounted EBITDA & 0.00 & 119.50 & 111.68 & 104.37 & 97.54 & 91.16 & 85.20 & 79.62 & 74.42 & 69.55 & 65.00 \\
Terminal Value & & & & & & & & & & \\
Upfront Capital Cost & -947.30 & & & & & & & & &
\end{tabular}

\section{ASSUMPTIONS:}

Algae oil content $=25 \%$

System productivity $=13.2 \mathrm{~g} / \mathrm{m} 2 /$ day

Total pond size $=4050 \mathrm{ha}$

COP of AJF $=\$ 2.59 / \mathrm{L}$

Total production $=39.31 \mathrm{ML} / \mathrm{Y}$

Total COP per year $=(39,310,000 \mathrm{~L} / \mathrm{Y}) \times(\$ 2.59 / \mathrm{L})=\$ 101,812,900$

Total Revenue from Sale of AJF per year $=(39,310,000 \mathrm{~L} / \mathrm{Y}) \times(\$ 0.84 / \mathrm{L})=\$ 33,020,400$

Average price of Jetfuel for $2013=\$ 132.80 / \mathrm{bbl}$ or $\$ 0.84 / \mathrm{L}$

Emissions price $=\$ 2000 / \mathrm{tCO} 2 \mathrm{e}$

Energy Density of Bio-SPK $=43.5 \mathrm{MJ} / \mathrm{kg}$

Algal oil denisty $=920 \mathrm{~kg} / \mathrm{m} 3$

Total production per year by energy $(\mathrm{MJ})=(39,310,000 \mathrm{~L}) \times(920 \mathrm{~kg} / \mathrm{m3}) \times(\mathrm{m} 3 / 1000 \mathrm{I}) \times(43.5 \mathrm{MJ} / \mathrm{kg})=1,573,186,200 \mathrm{MJ}$

Life-Cylce Emissions intensity of AJF $=25 \mathrm{gCO} e / \mathrm{MJ}$

Life-Cycle Emissions intensity of PJF $=87.5 \mathrm{gCO} e / \mathrm{MJ}$

Total Life-Cycle Emission per year from AJF $=(1,573,186,200 \mathrm{MJ}) \times(25 \mathrm{gCO} e / \mathrm{MJ})=39,329,655,000 \mathrm{MJ}$

Total Life-Cycle Emission per year from PJF $=(1,573,186,200 \mathrm{MJ}) \times(87.5 \mathrm{gCO} e / \mathrm{MJ})=137,653,792,500 \mathrm{gCO} \mathrm{e}$

Total Life-Cycle Emission benefit per year $=(137,653,792,500)-(39,329,655,000)=98,324,137,500$ or $98,324.14$ tCO2

Total Monetized Life-Cycle Emission Benefit per year $=(98,324.14 \mathrm{tCO} 2) \times(\$ 2000 / \mathrm{tCO})=\$ 196,648,280$

WACC $=7 \%$

Total UCC $=\$ 947.3 \mathrm{M}$ 


\section{APPENDIX D}

\section{Calculations for Changing JFP \& Carbon Tax - @ \$1/1 \& \$60/tC02e}

\begin{tabular}{lccccccccccc}
\multicolumn{1}{r}{ Source of Cash Flow (\$M) } & $\mathbf{0}$ & $\mathbf{1}$ & $\mathbf{2}$ & $\mathbf{3}$ & $\mathbf{4}$ & $\mathbf{5}$ & $\mathbf{6}$ & $\mathbf{7}$ & $\mathbf{8}$ & $\mathbf{9}$ & $\mathbf{1 0}$ \\
Total Revenue from sale of AJF & 0 & 39.31 & 39.31 & 39.31 & 39.31 & 39.31 & 39.31 & 39.31 & 39.31 & 39.31 & 39.31 \\
Total Revenue from emissions benefit & 0 & 5.90 & 5.90 & 5.90 & 5.90 & 5.90 & 5.90 & 5.90 & 5.90 & 5.90 & 5.90 \\
Total COP of AJF & 0 & 101.8 & 101.8 & 101.8 & 101.81 & 101.8 & 101.8 & 101.81 & 101.81 & 101.8 & 101.8 \\
EBITDA & 0 & -56.60 & -56.60 & -56.60 & -56.60 & -56.60 & -56.60 & -56.60 & -56.60 & -56.60 & -56.60 \\
Discount Factor & 1.00 & 1.07 & 1.14 & 1.23 & 1.31 & 1.40 & 1.50 & 1.61 & 1.72 & 1.84 & 1.97 \\
Discounted EBITDA & 0.00 & -52.90 & -49.44 & -46.20 & -43.18 & -40.36 & -37.71 & -35.25 & -32.94 & -30.79 & -28.77 \\
Terminal Value & & & & & & & & & & & \\
Upfront Capital Cost & -947.30 & & & & & & & & & &
\end{tabular}

\section{ASSUMPTIONS:}

Algae oil content $=25 \%$

System productivity $=13.2 \mathrm{~g} / \mathrm{m} 2 /$ day

Total pond size $=4050$ ha

COP of AJF $=\$ 2.59 / \mathrm{L}$

Total production $=39.31 \mathrm{ML} / \mathrm{Y}$

Total COP per year $=(39,310,000 \mathrm{~L} / \mathrm{Y}) \times(\$ 2.59 / \mathrm{L})=\$ 101,812,900$

Total Revenue from Sale of AJF per year $=(39,310,000 \mathrm{~L} / \mathrm{Y}) \times(\$ 1.00 / \mathrm{L})=\$ 39,310,000$

Hypothetical Jetfuel price $=\$ 1 / L(\$ 158.76 / \mathrm{bbl})$

Emissions price $=\$ 60 / \mathrm{tCO} 2 \mathrm{e}$

Energy Density of Bio-SPK $=43.5 \mathrm{MJ} / \mathrm{kg}$

Algal oil denisty $=920 \mathrm{~kg} / \mathrm{m} 3$

Total production per year by energy $(\mathrm{MJ})=(39,310,000 \mathrm{~L}) \times(920 \mathrm{~kg} / \mathrm{m3}) \times(\mathrm{m} 3 / 1000 \mathrm{l}) \times(43.5 \mathrm{MJ} / \mathrm{kg})=1,573,186,200 \mathrm{MJ}$

Life-Cylce Emissions intensity of AJF $=25 \mathrm{gCO} 2 \mathrm{e} / \mathrm{MJ}$

Life-Cycle Emissions intensity of PJF $=87.5 \mathrm{gCO} e / \mathrm{MJ}$

Total Life-Cycle Emission per year from AJF $=(1,573,186,200 \mathrm{MJ}) \times(25 \mathrm{gCO} e / \mathrm{MJ})=39,329,655,000 \mathrm{MJ}$

Total Life-Cycle Emission per year from PJF $=(1,573,186,200 \mathrm{MJ}) \times(87.5 \mathrm{gCO} \mathrm{e} / \mathrm{MJ})=137,653,792,500 \mathrm{gCO} \mathrm{e}$

Total Life-Cycle Emission benefit per year $=(137,653,792,500)-(39,329,655,000)=98,324,137,500$ or $98,324.14$ tCO2

Total Monetized Life-Cycle Emission Benefit per year $=(98,324.14 \mathrm{tCO}) \times(\$ 60 / \mathrm{tCO} 2)=\$ 5,899,448.25$

WACC $=7 \%$

Total UCC $=\$ 947.3 \mathrm{M}$ 


\section{Calculations for Changing JFP \& Carbon Tax - @ \$1/1 \& \$120/tCO2e}

\begin{tabular}{lccccccccccc}
\multicolumn{1}{r}{ Source of Cash Flow (\$M) } & $\mathbf{0}$ & $\mathbf{1}$ & $\mathbf{2}$ & $\mathbf{3}$ & $\mathbf{4}$ & $\mathbf{5}$ & $\mathbf{6}$ & $\mathbf{7}$ & $\mathbf{8}$ & $\mathbf{9}$ & $\mathbf{1 0}$ \\
Total Revenue from sale of AJF & 0 & 39.31 & 39.31 & 39.31 & 39.31 & 39.31 & 39.31 & 39.31 & 39.31 & 39.31 & 39.31 \\
Total Revenue from emissions benefit & 0 & 11.98 & 11.98 & 11.98 & 11.98 & 11.98 & 11.98 & 11.98 & 11.98 & 11.98 & 11.98 \\
Total COP of AJF & 0 & 101.8 & 101.8 & 101.8 & 101.81 & 101.8 & 101.8 & 101.81 & 101.81 & 101.8 & 101.8 \\
EBITDA & 0 & -50.52 & -50.52 & -50.52 & -50.52 & -50.52 & -50.52 & -50.52 & -50.52 & -50.52 & -50.52 \\
Discount Factor & 1.00 & 1.07 & 1.14 & 1.23 & 1.31 & 1.40 & 1.50 & 1.61 & 1.72 & 1.84 & 1.97 \\
Discounted EBITDA & 0.00 & -47.21 & -44.13 & -41.24 & -38.54 & -36.02 & -33.66 & -31.46 & -29.40 & -27.48 & -25.68 \\
Terminal Value & & & & & & & & & & & \\
Upfront Capital Cost & -947.30 & & & & & & & & & &
\end{tabular}

\section{ASSUMPTIONS:}

Algae oil content $=25 \%$

System productivity $=13.2 \mathrm{~g} / \mathrm{m} 2 /$ day

Total pond size $=4050$ ha

COP of AJF $=\$ 2.59 / \mathrm{L}$

Total production $=39.31 \mathrm{ML} / \mathrm{Y}$

Total COP per year $=(39,310,000 \mathrm{~L} / \mathrm{Y}) \times(\$ 2.59 / \mathrm{L})=\$ 101,812,900$

Total Revenue from Sale of AJF per year $=(39,310,000 \mathrm{~L} / \mathrm{Y}) \times(\$ 1.00 / \mathrm{L})=\$ 39,310,000$

Hypothetical Jetfuel price $=\$ 1 / \mathrm{L}(\$ 158.76 / \mathrm{bbl})$

Emissions price $=\$ 120 / \mathrm{tCO} \mathrm{e}$

Energy Density of Bio-SPK $=43.5 \mathrm{MJ} / \mathrm{kg}$

Algal oil denisty $=920 \mathrm{~kg} / \mathrm{m3}$

Total production per year by energy $(\mathrm{MJ})=(39,310,000 \mathrm{~L}) \times(920 \mathrm{~kg} / \mathrm{m} 3) \times(\mathrm{m} 3 / 1000 \mathrm{l}) \times(43.5 \mathrm{MJ} / \mathrm{kg})=1,573,186,200 \mathrm{MJ}$

Life-Cylce Emissions intensity of AJF $=25 \mathrm{gCO} e / \mathrm{MJ}$

Life-Cycle Emissions intensity of PJF $=87.5 \mathrm{gCO} 2 \mathrm{e} / \mathrm{MJ}$

Total Life-Cycle Emission per year from AJF $=(1,573,186,200 \mathrm{MJ}) \times(25 \mathrm{gCO} 2 \mathrm{e} / \mathrm{MJ})=39,329,655,000 \mathrm{MJ}$

Total Life-Cycle Emission per year from PJF $=(1,573,186,200 \mathrm{MJ}) \times(87.5 \mathrm{gCO} e / \mathrm{MJ})=137,653,792,500 \mathrm{gCO} \mathrm{e}$

Total Life-Cycle Emission benefit per year $=(137,653,792,500)-(39,329,655,000)=98,324,137,500$ or $98,324.14$ tCO2

Total Monetized Life-Cycle Emission Benefit per year $=(98,324.14$ tCO2 $) \times(\$ 120 / \mathrm{tCO})=\$ 11,978,896.50$

WACC $=7 \%$

Total UCC $=\$ 947.3 \mathrm{M}$ 


\section{Calculations for Changing JFP \& Carbon Tax - @ \$1/1 \& \$200/tCO2e}

\begin{tabular}{|c|c|c|c|c|c|c|c|c|c|c|c|}
\hline \multirow[b]{2}{*}{ Source of Cash Flow (\$M) } & \multicolumn{9}{|c|}{ Year (N) } & \multirow[b]{2}{*}{9} & \multirow[b]{2}{*}{10} \\
\hline & 0 & 1 & 2 & 3 & 4 & 5 & 6 & 7 & 8 & & \\
\hline Total Revenue from sale of AJF & 0 & 39.31 & 39.31 & 39.31 & 39.31 & 39.31 & 39.31 & 39.31 & 39.31 & 39.31 & 39.31 \\
\hline Total Revenue from emissions benefit & 0 & 19.66 & 19.66 & 19.66 & 19.66 & 19.66 & 19.66 & 19.66 & 19.66 & 19.66 & 19.66 \\
\hline Total COP of AJF & 0 & 101.8 & 101.8 & 101.8 & 101.81 & 101.8 & 101.8 & 101.81 & 101.81 & 101.8 & 101.8 \\
\hline EBITDA & 0 & -42.84 & -42.84 & -42.84 & -42.84 & -42.84 & -42.84 & -42.84 & -42.84 & -42.84 & -42.84 \\
\hline Discount Factor & 1.00 & 1.07 & 1.14 & 1.23 & 1.31 & 1.40 & 1.50 & 1.61 & 1.72 & 1.84 & 1.97 \\
\hline Discounted EBITDA & 0.00 & -40.04 & -37.42 & -34.97 & -32.68 & -30.54 & -28.55 & -26.68 & -24.93 & -23.30 & -21.78 \\
\hline Terminal Value & & & & & & & & & & & -612.00 \\
\hline Upfront Capital Cost & -947.30 & & & & & & & & & & \\
\hline NPV & -1860.19 & & & & & & & & & & \\
\hline
\end{tabular}

\section{ASSUMPTIONS:}

Algae oil content $=25 \%$

System productivity $=13.2 \mathrm{~g} / \mathrm{m} 2 /$ day

Total pond size $=4050$ ha

COP of AJF $=\$ 2.59 / \mathrm{L}$

Total production $=39.31 \mathrm{ML} / \mathrm{Y}$

Total COP per year $=(39,310,000 \mathrm{~L} / \mathrm{Y}) \times(\$ 2.59 / \mathrm{L})=\$ 101,812,900$

Total Revenue from Sale of AJF per year $=(39,310,000 \mathrm{~L} / \mathrm{Y}) \times(\$ 1.00 / \mathrm{L})=\$ 39,310,000$

Hypothetical Jetfuel price $=\$ 1 / L(\$ 158.76 / \mathrm{bbl})$

Emissions price $=\$ 200 / \mathrm{tCO} 2 \mathrm{e}$

Energy Density of Bio-SPK $=43.5 \mathrm{MJ} / \mathrm{kg}$

Algal oil denisty $=920 \mathrm{~kg} / \mathrm{m} 3$

Total production per year by energy $(\mathrm{MJ})=(39,310,000 \mathrm{~L}) \times(920 \mathrm{~kg} / \mathrm{m3}) \times(\mathrm{m3} / 1000 \mathrm{I}) \times(43.5 \mathrm{MJ} / \mathrm{kg})=1,573,186,200 \mathrm{MJ}$

Life-Cylce Emissions intensity of AJF $=25 \mathrm{gCO} 2 \mathrm{e} / \mathrm{MJ}$

Life-Cycle Emissions intensity of PJF $=87.5 \mathrm{gCO} 2 \mathrm{e} / \mathrm{MJ}$

Total Life-Cycle Emission per year from AJF $=(1,573,186,200 \mathrm{MJ}) \times(25 \mathrm{gCO} e / \mathrm{MJ})=39,329,655,000 \mathrm{MJ}$

Total Life-Cycle Emission per year from PJF $=(1,573,186,200 \mathrm{MJ}) \times(87.5 \mathrm{gCO} 2 \mathrm{e} / \mathrm{MJ})=137,653,792,500 \mathrm{gCO} \mathrm{e}$

Total Life-Cycle Emission benefit per year $=(137,653,792,500)-(39,329,655,000)=98,324,137,500$ or $98,324.14$ tCO2

Total Monetized Life-Cycle Emission Benefit per year $=(98,324.14 \mathrm{tCO} 2) \times(\$ 200 / \mathrm{tCO} 2)=\$ 19,664,827.50$

WACC $=7 \%$

Total UCC $=\$ 947.3 \mathrm{M}$ 


\section{Calculations for Changing JFP \& Carbon Tax - @ \$1/1 \& \$400/tCO2e}

\begin{tabular}{lccccccccccc}
\multicolumn{1}{r}{ Source of Cash Flow (\$M) } & $\mathbf{0}$ & $\mathbf{1}$ & $\mathbf{2}$ & $\mathbf{3}$ & $\mathbf{4}$ & $\mathbf{5}$ & $\mathbf{6}$ & $\mathbf{7}$ & $\mathbf{8}$ & $\mathbf{9}$ & $\mathbf{1 0}$ \\
Total Revenue from sale of AJF & 0 & 39.31 & 39.31 & 39.31 & 39.31 & 39.31 & 39.31 & 39.31 & 39.31 & 39.31 & 39.31 \\
Total Revenue from emissions benefit & 0 & 39.33 & 39.33 & 39.33 & 39.33 & 39.33 & 39.33 & 39.33 & 39.33 & 39.33 & 39.33 \\
Total COP of AJF & 0 & 101.8 & 101.8 & 101.8 & 101.81 & 101.8 & 101.8 & 101.81 & 101.81 & 101.8 & 101.8 \\
EBITDA & 0 & -23.17 & -23.17 & -23.17 & -23.17 & -23.17 & -23.17 & -23.17 & -23.17 & -23.17 & -23.17 \\
Discount Factor & 1.00 & 1.07 & 1.14 & 1.23 & 1.31 & 1.40 & 1.50 & 1.61 & 1.72 & 1.84 & 1.97 \\
Discounted EBITDA & 0.00 & -21.65 & -20.24 & -18.91 & -17.68 & -16.52 & -15.44 & -14.43 & -13.49 & -12.60 & -11.78 \\
Terminal Value & & & & & & & & & & & \\
Upfront Capital Cost & -947.30 & & & & & & & & & &
\end{tabular}

\section{ASSUMPTIONS:}

Algae oil content $=25 \%$

System productivity $=13.2 \mathrm{~g} / \mathrm{m} 2 /$ day

Total pond size $=4050$ ha

COP of AJF $=\$ 2.59 / \mathrm{L}$

Total production $=39.31 \mathrm{ML} / \mathrm{Y}$

Total COP per year $=(39,310,000 \mathrm{~L} / \mathrm{Y}) \times(\$ 2.59 / \mathrm{L})=\$ 101,812,900$

Total Revenue from Sale of AJF per year $=(39,310,000 \mathrm{~L} / \mathrm{Y}) \times(\$ 1.00 / \mathrm{L})=\$ 39,310,000$

Hypothetical Jetfuel price $=\$ 1 / \mathrm{L}(\$ 158.76 / \mathrm{bb})$

Emissions price $=\$ 400 / \mathrm{tCO} 2 \mathrm{e}$

Energy Density of Bio-SPK $=43.5 \mathrm{MJ} / \mathrm{kg}$

Algal oil denisty $=920 \mathrm{~kg} / \mathrm{m} 3$

Total production per year by energy $(\mathrm{MJ})=(39,310,000 \mathrm{~L}) \times(920 \mathrm{~kg} / \mathrm{m3}) \times(\mathrm{m} 3 / 1000 \mathrm{l}) \times(43.5 \mathrm{MJ} / \mathrm{kg})=1,573,186,200 \mathrm{MJ}$

Life-Cylce Emissions intensity of AJF $=25 \mathrm{gCO} e / \mathrm{MJ}$

Life-Cycle Emissions intensity of PJF $=87.5 \mathrm{gCO} e / \mathrm{MJ}$

Total Life-Cycle Emission per year from AJF $=(1,573,186,200 \mathrm{MJ}) \times(25 \mathrm{gCO} e / \mathrm{MJ})=39,329,655,000 \mathrm{MJ}$

Total Life-Cycle Emission per year from PJF $=(1,573,186,200 \mathrm{MJ}) \times(87.5 \mathrm{gCO} \mathrm{e} / \mathrm{MJ})=137,653,792,500 \mathrm{gCO} \mathrm{e}$

Total Life-Cycle Emission benefit per year $=(137,653,792,500)-(39,329,655,000)=98,324,137,500$ or $98,324.14$ tCO2

Total Monetized Life-Cycle Emission Benefit per year $=(98,324.14 \mathrm{tCO}) \times(\$ 400 / \mathrm{tCO})=\$ 39,329,656$

WACC $=7 \%$

Total UCC $=\$ 947.3 \mathrm{M}$ 


\section{Calculations for Changing JFP \& Carbon Tax - @ \$1/1 \& \$800/tCO2e}

\begin{tabular}{lccccccccccc}
\multicolumn{1}{r}{ Source of Cash Flow (\$M) } & $\mathbf{0}$ & $\mathbf{1}$ & $\mathbf{2}$ & $\mathbf{3}$ & $\mathbf{4}$ & $\mathbf{5}$ & $\mathbf{6}$ & $\mathbf{7}$ & $\mathbf{8}$ & $\mathbf{9}$ & $\mathbf{1 0}$ \\
Total Revenue from sale of AJF & 0 & 39.31 & 39.31 & 39.31 & 39.31 & 39.31 & 39.31 & 39.31 & 39.31 & 39.31 & 39.31 \\
Total Revenue from emissions benefit & 0 & 78.66 & 78.66 & 78.66 & 78.66 & 78.66 & 78.66 & 78.66 & 78.66 & 78.66 & 78.66 \\
Total COP of AJF & 0 & 101.8 & 101.8 & 101.8 & 101.81 & 101.8 & 101.8 & 101.81 & 101.81 & 101.8 & 101.8 \\
EBITDA & 0 & 16.16 & 16.16 & 16.16 & 16.16 & 16.16 & 16.16 & 16.16 & 16.16 & 16.16 & 16.16 \\
Discount Factor & 1.00 & 1.07 & 1.14 & 1.23 & 1.31 & 1.40 & 1.50 & 1.61 & 1.72 & 1.84 & 1.97 \\
Discounted EBITDA & 0.00 & 15.10 & 14.11 & 13.19 & 12.33 & 11.52 & 10.77 & 10.06 & 9.41 & 8.79 & 8.21 \\
Terminal Value & & & & & & & & & & & \\
Upfront Capital Cost & -947.30 & & & & & & & & & &
\end{tabular}

\section{ASSUMPTIONS}

Algae oil content $=25 \%$

System productivity $=13.2 \mathrm{~g} / \mathrm{m} 2 /$ day

Total pond size $=4050$ ha

COP of AJF $=\$ 2.59 / \mathrm{L}$

Total production $=39.31 \mathrm{ML} / \mathrm{Y}$

Total COP per year $=(39,310,000 \mathrm{~L} / \mathrm{Y}) \times(\$ 2.59 / \mathrm{L})=\$ 101,812,900$

Total Revenue from Sale of AJF per year $=(39,310,000 \mathrm{~L} / \mathrm{Y}) \times(\$ 1.00 / \mathrm{L})=\$ 39,310,000$

Hypothetical Jetfuel price $=\$ 1 / \mathrm{L}(\$ 158.76 / \mathrm{bbl})$

Emissions price $=\$ 800 / \mathrm{tCO} 2 \mathrm{e}$

Energy Density of Bio-SPK $=43.5 \mathrm{MJ} / \mathrm{kg}$

Algal oil denisty $=920 \mathrm{~kg} / \mathrm{m} 3$

Total production per year by energy $(\mathrm{MJ})=(39,310,000 \mathrm{~L}) \times(920 \mathrm{~kg} / \mathrm{m3}) \times(\mathrm{m3} / 1000 \mathrm{I}) \times(43.5 \mathrm{MJ} / \mathrm{kg})=1,573,186,200 \mathrm{MJ}$

Life-Cylce Emissions intensity of AJF $=25 \mathrm{gCO} 2 \mathrm{e} / \mathrm{MJ}$

Life-Cycle Emissions intensity of PJF $=87.5 \mathrm{gCO} 2 \mathrm{e} / \mathrm{MJ}$

Total Life-Cycle Emission per year from AJF $=(1,573,186,200 \mathrm{MJ}) \times(25 \mathrm{gCO} e / \mathrm{MJ})=39,329,655,000 \mathrm{MJ}$

Total Life-Cycle Emission per year from PJF $=(1,573,186,200 \mathrm{MJ}) \times(87.5 \mathrm{gCO} 2 \mathrm{e} / \mathrm{MJ})=137,653,792,500 \mathrm{gCO} \mathrm{e}$

Total Life-Cycle Emission benefit per year $=(137,653,792,500)-(39,329,655,000)=98,324,137,500$ or $98,324.14$ tCO2

Total Monetized Life-Cycle Emission Benefit per year $=(98,324.14 \mathrm{tCO} 2) \times(\$ 800 / \mathrm{tCO})=\$ 78,659,312$

WACC $=7 \%$

Total UCC $=\$ 947.3 \mathrm{M}$ 


\section{Calculations for Changing JFP \& Carbon Tax - @ \$1/1 \& \$2000/tC02e}

\begin{tabular}{lccccccccccc}
\multicolumn{1}{r}{ Source of Cash Flow (\$M) } & $\mathbf{0}$ & $\mathbf{1}$ & $\mathbf{2}$ & $\mathbf{3}$ & $\mathbf{4}$ & $\mathbf{5}$ & $\mathbf{6}$ & $\mathbf{7}$ & $\mathbf{8}$ & $\mathbf{9}$ & $\mathbf{1 0}$ \\
Total Revenue from sale of AJF & 0 & 39.31 & 39.31 & 39.31 & 39.31 & 39.31 & 39.31 & 39.31 & 39.31 & 39.31 & 39.31 \\
Total Revenue from emissions benefit & 0 & 196.65 & 196.65 & 196.65 & 196.65 & 196.65 & 196.65 & 196.65 & 196.65 & 196.65 & 196.65 \\
Total COP of AJF & 0 & 101.8 & 101.8 & 101.8 & 101.81 & 101.8 & 101.8 & 101.81 & 101.81 & 101.8 & 101.8 \\
EBITDA & 0 & 134.15 & 134.15 & 134.15 & 134.15 & 134.15 & 134.15 & 134.15 & 134.15 & 134.15 & 134.15 \\
Discount Factor & 1.00 & 1.07 & 1.14 & 1.23 & 1.31 & 1.40 & 1.50 & 1.61 & 1.72 & 1.84 & 1.97 \\
Discounted EBITDA & 0.00 & 125.37 & 117.17 & 109.51 & 102.34 & 95.65 & 89.39 & 83.54 & 78.08 & 72.97 & 68.20 \\
Terminal Value & & & & & & & & & & & \\
Upfront Capital Cost & -947.30 & & & & & & & & & &
\end{tabular}

\section{ASSUMPTIONS:}

Algae oil content $=25 \%$

System productivity $=13.2 \mathrm{~g} / \mathrm{m} 2 /$ day

Total pond size $=4050$ ha

COP of AJF $=\$ 2.59 / \mathrm{L}$

Total production $=39.31 \mathrm{ML} / \mathrm{Y}$

Total COP per year $=(39,310,000 \mathrm{~L} / \mathrm{Y}) \times(\$ 2.59 / \mathrm{L})=\$ 101,812,900$

Total Revenue from Sale of AJF per year $=(39,310,000 \mathrm{~L} / \mathrm{Y}) \times(\$ 1.00 / \mathrm{L})=\$ 39,310,000$

Hypothetical Jetfuel price $=\$ 1 / \mathrm{L}(\$ 158.76 / \mathrm{bbl})$

Emissions price $=\$ 2000 / \mathrm{tCO} 2 \mathrm{e}$

Energy Density of Bio-SPK $=43.5 \mathrm{MJ} / \mathrm{kg}$

Algal oil denisty $=920 \mathrm{~kg} / \mathrm{m} 3$

Total production per year by energy $(\mathrm{MJ})=(39,310,000 \mathrm{~L}) \times(920 \mathrm{~kg} / \mathrm{m} 3) \times(\mathrm{m} 3 / 1000 \mathrm{l}) \times(43.5 \mathrm{MJ} / \mathrm{kg})=1,573,186,200 \mathrm{MJ}$

Life-Cylce Emissions intensity of AJF $=25 \mathrm{gCO} e / \mathrm{MJ}$

Life-Cycle Emissions intensity of PJF $=87.5 \mathrm{gCO} 2 \mathrm{e} / \mathrm{MJ}$

Total Life-Cycle Emission per year from AJF $=(1,573,186,200 \mathrm{MJ}) \times(25 \mathrm{gCO} 2 \mathrm{e} / \mathrm{MJ})=39,329,655,000 \mathrm{MJ}$

Total Life-Cycle Emission per year from PJF $=(1,573,186,200 \mathrm{MJ}) \times(87.5 \mathrm{gCO} e / \mathrm{MJ})=137,653,792,500 \mathrm{gCO} \mathrm{e}$

Total Life-Cycle Emission benefit per year $=(137,653,792,500)-(39,329,655,000)=98,324,137,500$ or $98,324.14$ tCO2

Total Monetized Life-Cycle Emission Benefit per year $=(98,324.14$ tCO2 $) \times(\$ 2000 / \mathrm{tCO})=\$ 196,648,280$

WACC $=7 \%$

Total UCC $=\$ 947.3 \mathrm{M}$ 


\section{Calculations for Changing JFP \& Carbon Tax - @ \$1.50/1 \& \$60/tCO2e}

\begin{tabular}{lccccccccccc}
\multicolumn{1}{r}{ Source of Cash Flow (\$M) } & $\mathbf{0}$ & $\mathbf{1}$ & $\mathbf{2}$ & $\mathbf{3}$ & $\mathbf{4}$ & $\mathbf{5}$ & $\mathbf{6}$ & $\mathbf{7}$ & $\mathbf{8}$ & $\mathbf{9}$ & $\mathbf{1 0}$ \\
Total Revenue from sale of AJF & 0 & 58.97 & 58.97 & 58.97 & 58.97 & 58.97 & 58.97 & 58.97 & 58.97 & 58.97 & 58.97 \\
Total Revenue from emissions benefit & 0 & 5.90 & 5.90 & 5.90 & 5.90 & 5.90 & 5.90 & 5.90 & 5.90 & 5.90 & 5.90 \\
Total COP of AJF & 0 & 101.8 & 101.8 & 101.8 & 101.81 & 101.8 & 101.8 & 101.81 & 101.81 & 101.8 & 101.8 \\
EBITDA & 0 & -36.94 & -36.94 & -36.94 & -36.94 & -36.94 & -36.94 & -36.94 & -36.94 & -36.94 & -36.94 \\
Discount Factor & 1.00 & 1.07 & 1.14 & 1.23 & 1.31 & 1.40 & 1.50 & 1.61 & 1.72 & 1.84 & 1.97 \\
Discounted EBITDA & 0.00 & -34.52 & -32.26 & -30.15 & -28.18 & -26.34 & -24.61 & -23.00 & -21.50 & -20.09 & -18.78 \\
Terminal Value & & & & & & & & & & & \\
Upfront Capital Cost & -947.30 & & & & & & & & & &
\end{tabular}

\section{ASSUMPTIONS:}

Algae oil content $=25 \%$

System productivity $=13.2 \mathrm{~g} / \mathrm{m} 2 /$ day

Total pond size $=4050$ ha

COP of AJF $=\$ 2.59 / \mathrm{L}$

Total production $=39.31 \mathrm{ML} / \mathrm{Y}$

Total COP per year $=(39,310,000 \mathrm{~L} / \mathrm{Y}) \times(\$ 2.59 / \mathrm{L})=\$ 101,812,900$

Total Revenue from Sale of AJF per year $=(39,310,000 \mathrm{~L} / \mathrm{Y}) \times(\$ 1.50 / \mathrm{L})=\$ 58,965,000$

Hypothetical Jetfuel price $=\$ 1.50 / \mathrm{L}(\$ 238.76 / \mathrm{bbl})$

Emissions price $=\$ 60 / \mathrm{tCO} 2 \mathrm{e}$

Energy Density of Bio-SPK $=43.5 \mathrm{MJ} / \mathrm{kg}$

Algal oil denisty $=920 \mathrm{~kg} / \mathrm{m} 3$

Total production per year by energy $(\mathrm{MJ})=(39,310,000 \mathrm{~L}) \times(920 \mathrm{~kg} / \mathrm{m3}) \times(\mathrm{m} 3 / 1000 \mathrm{I}) \times(43.5 \mathrm{MJ} / \mathrm{kg})=1,573,186,200 \mathrm{MJ}$

Life-Cylce Emissions intensity of AJF $=25 \mathrm{gCO} 2 \mathrm{e} / \mathrm{MJ}$

Life-Cycle Emissions intensity of PJF $=87.5 \mathrm{gCO} e / \mathrm{MJ}$

Total Life-Cycle Emission per year from AJF $=(1,573,186,200 \mathrm{MJ}) \times(25 \mathrm{gCO} 2 \mathrm{e} / \mathrm{MJ})=39,329,655,000 \mathrm{MJ}$

Total Life-Cycle Emission per year from PJF $=(1,573,186,200 \mathrm{MJ}) \times(87.5 \mathrm{gCO} e / \mathrm{MJ})=137,653,792,500 \mathrm{gCO} e$

Total Life-Cycle Emission benefit per year $=(137,653,792,500)-(39,329,655,000)=98,324,137,500$ or $98,324.14$ tCO2

Total Monetized Life-Cycle Emission Benefit per year $=(98,324.14 \mathrm{tCO}) \times(\$ 60 / \mathrm{tCO})=\$ 5,899,448.25$

WACC $=7 \%$

Total UCC $=\$ 947.3 \mathrm{M}$ 


\section{Calculations for Changing JFP \& Carbon Tax - @ \$1.50/1 \& \$120/tC02e}

\begin{tabular}{|c|c|c|c|c|c|c|c|c|c|c|c|}
\hline \multirow[b]{2}{*}{ Source of Cash Flow (\$M) } & \multicolumn{9}{|c|}{ Year (N) } & \multirow[b]{2}{*}{9} & \multirow[b]{2}{*}{10} \\
\hline & 0 & 1 & 2 & 3 & 4 & 5 & 6 & 7 & 8 & & \\
\hline Total Revenue from emissions benefit & 0 & 11.98 & 11.98 & 11.98 & 11.98 & 11.98 & 11.98 & 11.98 & 11.98 & 11.98 & 11.98 \\
\hline EBITDA & 0 & -30.86 & -30.86 & -30.86 & -30.86 & -30.86 & -30.86 & -30.86 & -30.86 & -30.86 & -30.86 \\
\hline Discount Factor & 1.00 & 1.07 & 1.14 & 1.23 & 1.31 & 1.40 & 1.50 & 1.61 & 1.72 & 1.84 & 1.97 \\
\hline Discounted EBITDA & 0.00 & -28.84 & -26.95 & -25.19 & -23.54 & -22.00 & -20.56 & -19.22 & -17.96 & -16.79 & -15.69 \\
\hline
\end{tabular}

Upfront Capital Cost $\quad-947.30$

NPV

$-1604.90$

\section{ASSUMPTIONS:}

Algae oil content $=25 \%$

System productivity $=13.2 \mathrm{~g} / \mathrm{m} 2 /$ day

Total pond size $=4050$ ha

COP of AJF $=\$ 2.59 / \mathrm{L}$

Total production $=39.31 \mathrm{ML} / \mathrm{Y}$

Total COP per year $=(39,310,000 \mathrm{~L} / \mathrm{Y}) \times(\$ 2.59 / \mathrm{L})=\$ 101,812,900$

Total Revenue from Sale of AJF per year $=(39,310,000 \mathrm{~L} / \mathrm{Y}) \times(\$ 1.50 / \mathrm{L})=\$ 58,965,000$

Hypothetical Jetfuel price $=\$ 1.50 / \mathrm{L}(\$ 238.76 / \mathrm{bbl})$

Emissions price $=\$ 120 / \mathrm{tCO} 2 \mathrm{e}$

Energy Density of Bio-SPK $=43.5 \mathrm{MJ} / \mathrm{kg}$

Algal oil denisty $=920 \mathrm{~kg} / \mathrm{m} 3$

Total production per year by energy $(\mathrm{MJ})=(39,310,000 \mathrm{~L}) \times(920 \mathrm{~kg} / \mathrm{m} 3) \times(\mathrm{m} 3 / 1000 \mathrm{I}) \times(43.5 \mathrm{MJ} / \mathrm{kg})=1,573,186,200 \mathrm{MJ}$

Life-Cylce Emissions intensity of AJF $=25 \mathrm{gCO} 2 \mathrm{e} / \mathrm{MJ}$

Life-Cycle Emissions intensity of PJF $=87.5 \mathrm{gCO} 2 \mathrm{e} / \mathrm{MJ}$

Total Life-Cycle Emission per year from AJF $=(1,573,186,200 \mathrm{MJ}) \times(25 \mathrm{gCO} e / \mathrm{MJ})=39,329,655,000 \mathrm{MJ}$

Total Life-Cycle Emission per year from PJF $=(1,573,186,200 \mathrm{MJ}) \times(87.5 \mathrm{gCO} e / \mathrm{MJ})=137,653,792,500 \mathrm{gCO} \mathrm{e}$

Total Life-Cycle Emission benefit per year $=(137,653,792,500)-(39,329,655,000)=98,324,137,500$ or $98,324.14$ tCO2

Total Monetized Life-Cycle Emission Benefit per year $=(98,324.14 \mathrm{tCO} 2) \times(\$ 120 / \mathrm{tCO})=\$ 11,978,896.50$

WACC $=7 \%$

Total UCC $=\$ 947.3 \mathrm{M}$ 


\section{Calculations for Changing JFP \& Carbon Tax - @ \$1.50/1 \& \$200/tC02e}

\begin{tabular}{lccccccccccc}
\multicolumn{1}{r}{ Source of Cash Flow (\$M) } & $\mathbf{0}$ & $\mathbf{1}$ & $\mathbf{2}$ & $\mathbf{3}$ & $\mathbf{4}$ & $\mathbf{5}$ & $\mathbf{6}$ & $\mathbf{7}$ & $\mathbf{8}$ & $\mathbf{9}$ & $\mathbf{1 0}$ \\
Total Revenue from sale of AJF & 0 & 58.97 & 58.97 & 58.97 & 58.97 & 58.97 & 58.97 & 58.97 & 58.97 & 58.97 & 58.97 \\
Total Revenue from emissions benefit & 0 & 19.66 & 19.66 & 19.66 & 19.66 & 19.66 & 19.66 & 19.66 & 19.66 & 19.66 & 19.66 \\
Total COP of AJF & 0 & 101.8 & 101.8 & 101.8 & 101.81 & 101.8 & 101.8 & 101.81 & 101.81 & 101.8 & 101.8 \\
EBITDA & 0 & -23.18 & -23.18 & -23.18 & -23.18 & -23.18 & -23.18 & -23.18 & -23.18 & -23.18 & -23.18 \\
Discount Factor & 1.00 & 1.07 & 1.14 & 1.23 & 1.31 & 1.40 & 1.50 & 1.61 & 1.72 & 1.84 & 1.97 \\
Discounted EBITDA & 0.00 & -21.66 & -20.25 & -18.92 & -17.68 & -16.53 & -15.45 & -14.44 & -13.49 & -12.61 & -11.78 \\
Terminal Value & & & & & & & & & & & \\
Upfront Capital Cost & -947.30 & & & & & & & & & &
\end{tabular}

\section{ASSUMPTIONS:}

Algae oil content $=25 \%$

System productivity $=13.2 \mathrm{~g} / \mathrm{m} 2 /$ day

Total pond size $=4050$ ha

COP of AJF $=\$ 2.59 / \mathrm{L}$

Total production $=39.31 \mathrm{ML} / \mathrm{Y}$

Total COP per year $=(39,310,000 \mathrm{~L} / \mathrm{Y}) \times(\$ 2.59 / \mathrm{L})=\$ 101,812,900$

Total Revenue from Sale of AJF per year $=(39,310,000 \mathrm{~L} / \mathrm{Y}) \times(\$ 1.50 / \mathrm{L})=\$ 58,965,000$

Hypothetical Jetfuel price $=\$ 1.50 / \mathrm{L}(\$ 238.76 / \mathrm{bbl})$

Emissions price $=\$ 200 / \mathrm{tCO} \mathrm{e}$

Energy Density of Bio-SPK $=43.5 \mathrm{MJ} / \mathrm{kg}$

Algal oil denisty $=920 \mathrm{~kg} / \mathrm{m} 3$

Total production per year by energy $(\mathrm{MJ})=(39,310,000 \mathrm{~L}) \times(920 \mathrm{~kg} / \mathrm{m3}) \times(\mathrm{m} 3 / 1000 \mathrm{I}) \times(43.5 \mathrm{MJ} / \mathrm{kg})=1,573,186,200 \mathrm{MJ}$

Life-Cylce Emissions intensity of AJF $=25 \mathrm{gCO} e / \mathrm{MJ}$

Life-Cycle Emissions intensity of PJF $=87.5 \mathrm{gCO} 2 \mathrm{e} / \mathrm{MJ}$

Total Life-Cycle Emission per year from AJF $=(1,573,186,200 \mathrm{MJ}) \times(25 \mathrm{gCO} e / \mathrm{MJ})=39,329,655,000 \mathrm{MJ}$

Total Life-Cycle Emission per year from PJF $=(1,573,186,200 \mathrm{MJ}) \times(87.5 \mathrm{gCO} \mathrm{e} / \mathrm{MJ})=137,653,792,500 \mathrm{gCO} \mathrm{e}$

Total Life-Cycle Emission benefit per year $=(137,653,792,500)-(39,329,655,000)=98,324,137,500$ or $98,324.14$ tCO2

Total Monetized Life-Cycle Emission Benefit per year $=(98,324.14 \mathrm{tCO} 2) \times(\$ 200 / \mathrm{tCO})=\$ 19,664,827.50$

WACC $=7 \%$

Total UCC $=\$ 947.3 \mathrm{M}$ 


\section{Calculations for Changing JFP \& Carbon Tax - @ \$1.50/1 \& \$400/tC02e}

\begin{tabular}{lcccccccccc}
\multicolumn{1}{r}{ Source of Cash Flow (\$M) } & $\mathbf{9}$ & $\mathbf{1}$ & $\mathbf{2}$ & $\mathbf{3}$ & $\mathbf{4}$ & $\mathbf{5}$ & $\mathbf{6}$ & $\mathbf{7}$ & $\mathbf{8}$ & $\mathbf{9}$ \\
Total Revenue from sale of AJF & 0 & 58.97 & 58.97 & 58.97 & 58.97 & 58.97 & 58.97 & 58.97 & 58.97 & 58.97 \\
Total Revenue from emissions benefit & 0 & 39.33 & 39.33 & 39.33 & 39.33 & 39.33 & 39.33 & 39.33 & 39.33 & 39.33 \\
Total COP of AJF & 0 & 101.8 & 101.8 & 101.8 & 101.81 & 101.8 & 101.8 & 101.81 & 101.81 & 101.8 \\
EBITDA & 0 & -3.51 & -3.51 & -3.51 & -3.51 & -3.51 & -3.51 & -3.51 & -3.51 & -3.51 \\
Discount Factor & 1.00 & 1.07 & 1.14 & 1.23 & 1.31 & 1.40 & 1.50 & 1.61 & 1.72 & 1.84 \\
Discounted EBITDA & 0.00 & -3.28 & -3.07 & -2.87 & -2.68 & -2.50 & -2.34 & -2.19 & -2.04 & -1.91 \\
Terminal Value & & & & & & & & & & \\
Upfront Capital Cost & -947.30 & & & & & & & & &
\end{tabular}

\section{ASSUMPTIONS:}

Algae oil content $=25 \%$

System productivity $=13.2 \mathrm{~g} / \mathrm{m} 2 /$ day

Total pond size $=4050$ ha

COP of AJF $=\$ 2.59 / \mathrm{L}$

Total production $=39.31 \mathrm{ML} / \mathrm{Y}$

Total COP per year $=(39,310,000 \mathrm{~L} / \mathrm{Y}) \times(\$ 2.59 / \mathrm{L})=\$ 101,812,900$

Total Revenue from Sale of AJF per year $=(39,310,000 \mathrm{~L} / \mathrm{Y}) \times(\$ 1.50 / \mathrm{L})=\$ 58,965,000$

Hypothetical Jetfuel price $=\$ 1.50 / \mathrm{L}(\$ 238.76 / \mathrm{bbl})$

Emissions price $=\$ 400 / \mathrm{tCO} 2 \mathrm{e}$

Energy Density of Bio-SPK $=43.5 \mathrm{MJ} / \mathrm{kg}$

Algal oil denisty $=920 \mathrm{~kg} / \mathrm{m} 3$

Total production per year by energy $(\mathrm{MJ})=(39,310,000 \mathrm{~L}) \times(920 \mathrm{~kg} / \mathrm{m3}) \times(\mathrm{m3} / 1000 \mathrm{I}) \times(43.5 \mathrm{MJ} / \mathrm{kg})=1,573,186,200 \mathrm{MJ}$

Life-Cylce Emissions intensity of AJF $=25 \mathrm{gCO} 2 \mathrm{e} / \mathrm{MJ}$

Life-Cycle Emissions intensity of PJF $=87.5 \mathrm{gCO} e / \mathrm{MJ}$

Total Life-Cycle Emission per year from AJF $=(1,573,186,200 \mathrm{MJ}) \times(25 \mathrm{gCO} e / \mathrm{MJ})=39,329,655,000 \mathrm{MJ}$

Total Life-Cycle Emission per year from PJF $=(1,573,186,200 \mathrm{MJ}) \times(87.5 \mathrm{gCO} e / \mathrm{MJ})=137,653,792,500 \mathrm{gCO} e$

Total Life-Cycle Emission benefit per year $=(137,653,792,500)-(39,329,655,000)=98,324,137,500$ or $98,324.14$ tCO2

Total Monetized Life-Cycle Emission Benefit per year $=(98,324.14 \mathrm{tCO}) \times(\$ 400 / \mathrm{tCO})=\$ 39,329,656$

WACC $=7 \%$

Total UCC $=\$ 947.3 \mathrm{M}$ 


\section{Calculations for Changing JFP \& Carbon Tax - @ \$1.50/1 \& \$800/tC02e}

\begin{tabular}{lccccccccccc}
\multicolumn{1}{c}{ Source of Cash Flow (\$M) } & $\mathbf{0}$ & $\mathbf{1}$ & $\mathbf{2}$ & $\mathbf{3}$ & $\mathbf{4}$ & $\mathbf{5}$ & $\mathbf{6}$ & $\mathbf{7}$ & $\mathbf{8}$ & $\mathbf{9}$ & $\mathbf{1 0}$ \\
Total Revenue from sale of AJF & 0 & 58.97 & 58.97 & 58.97 & 58.97 & 58.97 & 58.97 & 58.97 & 58.97 & 58.97 & 58.97 \\
Total Revenue from emissions benefit & 0 & 78.66 & 78.66 & 78.66 & 78.66 & 78.66 & 78.66 & 78.66 & 78.66 & 78.66 & 78.66 \\
Total COP of AJF & 0 & 101.8 & 101.8 & 101.8 & 101.81 & 101.8 & 101.8 & 101.81 & 101.81 & 101.8 & 101.8 \\
EBITDA & 0 & 35.82 & 35.82 & 35.82 & 35.82 & 35.82 & 35.82 & 35.82 & 35.82 & 35.82 & 35.82 \\
Discount Factor & 1.00 & 1.07 & 1.14 & 1.23 & 1.31 & 1.40 & 1.50 & 1.61 & 1.72 & 1.84 & 1.97 \\
Discounted EBITDA & 0.00 & 33.48 & 31.29 & 29.24 & 27.33 & 25.54 & 23.87 & 22.31 & 20.85 & 19.48 & 18.21 \\
Terminal Value & & & & & & & & &
\end{tabular}

Upfront Capital Cost $\quad-947.30$

NPV

$-184.00$

\section{ASSUMPTIONS:}

Algae oil content $=25 \%$

System productivity $=13.2 \mathrm{~g} / \mathrm{m} 2 /$ day

Total pond size $=4050$ ha

COP of AJF $=\$ 2.59 / \mathrm{L}$

Total production $=39.31 \mathrm{ML} / \mathrm{Y}$

Total COP per year $=(39,310,000 \mathrm{~L} / \mathrm{Y}) \times(\$ 2.59 / \mathrm{L})=\$ 101,812,900$

Total Revenue from Sale of AJF per year $=(39,310,000 \mathrm{~L} / \mathrm{Y}) \times(\$ 1.50 / \mathrm{L})=\$ 58,965,000$

Hypothetical Jetfuel price $=\$ 1.50 / \mathrm{L}(\$ 238.76 / \mathrm{bbl})$

Emissions price $=\$ 800 / \mathrm{tCO} 2 \mathrm{e}$

Energy Density of Bio-SPK $=43.5 \mathrm{MJ} / \mathrm{kg}$

Algal oil denisty $=920 \mathrm{~kg} / \mathrm{m} 3$

Total production per year by energy $(\mathrm{MJ})=(39,310,000 \mathrm{~L}) \times(920 \mathrm{~kg} / \mathrm{m3}) \times(\mathrm{m3} / 1000 \mathrm{I}) \times(43.5 \mathrm{MJ} / \mathrm{kg})=1,573,186,200 \mathrm{MJ}$

Life-Cylce Emissions intensity of AJF $=25 \mathrm{gCO} 2 \mathrm{e} / \mathrm{MJ}$

Life-Cycle Emissions intensity of PJF $=87.5 \mathrm{gCO} 2 \mathrm{e} / \mathrm{MJ}$

Total Life-Cycle Emission per year from AJF $=(1,573,186,200 \mathrm{MJ}) \times(25 \mathrm{gCO} e / \mathrm{MJ})=39,329,655,000 \mathrm{MJ}$

Total Life-Cycle Emission per year from PJF $=(1,573,186,200 \mathrm{MJ}) \times(87.5 \mathrm{gCO} e / \mathrm{MJ})=137,653,792,500 \mathrm{gCO} e$

Total Life-Cycle Emission benefit per year $=(137,653,792,500)-(39,329,655,000)=98,324,137,500$ or $98,324.14$ tCO2

Total Monetized Life-Cycle Emission Benefit per year $=(98,324.14 \mathrm{tCO}) \times(\$ 800 / \mathrm{tCO} 2)=\$ 78,659,312$

WACC $=7 \%$

Total UCC $=\$ 947.3 \mathrm{M}$ 


\section{Calculations for Changing JFP \& Carbon Tax - @ \$1.50/1 \& \$2000/tCO2e}

\begin{tabular}{lccccccccccc}
\multicolumn{1}{r}{ Source of Cash Flow (\$M) } & $\mathbf{0}$ & $\mathbf{1}$ & $\mathbf{2}$ & $\mathbf{3}$ & $\mathbf{4}$ & $\mathbf{5}$ & $\mathbf{6}$ & $\mathbf{7}$ & $\mathbf{8}$ & $\mathbf{9}$ & $\mathbf{1 0}$ \\
Total Revenue from sale of AJF & 0 & 58.97 & 58.97 & 58.97 & 58.97 & 58.97 & 58.97 & 58.97 & 58.97 & 58.97 & 58.97 \\
Total Revenue from emissions benefit & 0 & 196.65 & 196.65 & 196.65 & 196.65 & 196.65 & 196.65 & 196.65 & 196.65 & 196.65 & 196.65 \\
Total COP of AJF & 0 & 101.8 & 101.8 & 101.8 & 101.81 & 101.8 & 101.8 & 101.81 & 101.81 & 101.8 & 101.8 \\
EBITDA & 0 & 153.81 & 153.81 & 153.81 & 153.81 & 153.81 & 153.81 & 153.81 & 153.81 & 153.81 & 153.81 \\
Discount Factor & 1.00 & 1.07 & 1.14 & 1.23 & 1.31 & 1.40 & 1.50 & 1.61 & 1.72 & 1.84 & 1.97 \\
Discounted EBITDA & 0.00 & 143.75 & 134.34 & 125.55 & 117.34 & 109.66 & 102.49 & 95.79 & 89.52 & 83.66 & 78.19 \\
Terminal Value & & & & & & & & & & & \\
Upfront Capital Cost & -947.30 & & & & & & & & & &
\end{tabular}

\section{ASSUMPTIONS:}

Algae oil content $=25 \%$

System productivity $=13.2 \mathrm{~g} / \mathrm{m} 2 /$ day

Total pond size $=4050$ ha

COP of AJF $=\$ 2.59 / \mathrm{L}$

Total production $=39.31 \mathrm{ML} / \mathrm{Y}$

Total COP per year $=(39,310,000 \mathrm{~L} / \mathrm{Y}) \times(\$ 2.59 / \mathrm{L})=\$ 101,812,900$

Total Revenue from Sale of AJF per year $=(39,310,000 \mathrm{~L} / \mathrm{Y}) \times(\$ 1.50 / \mathrm{L})=\$ 58,965,000$

Hypothetical Jetfuel price $=\$ 1.50 / \mathrm{L}(\$ 238.76 / \mathrm{bbl})$

Emissions price $=\$ 2000 / \mathrm{tCO} 2 \mathrm{e}$

Energy Density of Bio-SPK $=43.5 \mathrm{MJ} / \mathrm{kg}$

Algal oil denisty $=920 \mathrm{~kg} / \mathrm{m} 3$

Total production per year by energy $(\mathrm{MJ})=(39,310,000 \mathrm{~L}) \times(920 \mathrm{~kg} / \mathrm{m3}) \times(\mathrm{m3} / 1000 \mathrm{l}) \times(43.5 \mathrm{MJ} / \mathrm{kg})=1,573,186,200 \mathrm{MJ}$

Life-Cylce Emissions intensity of AJF $=25 \mathrm{gCO} 2 \mathrm{e} / \mathrm{MJ}$

Life-Cycle Emissions intensity of PJF $=87.5 \mathrm{gCO} e / \mathrm{MJ}$

Total Life-Cycle Emission per year from AJF $=(1,573,186,200 \mathrm{MJ}) \times(25 \mathrm{gCO} e / \mathrm{MJ})=39,329,655,000 \mathrm{MJ}$

Total Life-Cycle Emission per year from PJF $=(1,573,186,200 \mathrm{MJ}) \times(87.5 \mathrm{gCO} 2 \mathrm{e} / \mathrm{MJ})=137,653,792,500 \mathrm{gCO} e$

Total Life-Cycle Emission benefit per year $=(137,653,792,500)-(39,329,655,000)=98,324,137,500$ or $98,324.14$ tCO2

Total Monetized Life-Cycle Emission Benefit per year $=(98,324.14 \mathrm{tCO}) \times(\$ 2000 / \mathrm{tCO} 2)=\$ 196,648,280$

WACC $=7 \%$

Total UCC $=\$ 947.3 \mathrm{M}$ 


\section{Calculations for Changing JFP \& Carbon Tax - @ \$3/1 \& \$60/tC02e}

\begin{tabular}{lccccccccccc}
\multicolumn{1}{r}{ Source of Cash Flow (\$M) } & $\mathbf{0}$ & $\mathbf{1}$ & $\mathbf{2}$ & $\mathbf{3}$ & $\mathbf{4}$ & $\mathbf{5}$ & $\mathbf{6}$ & $\mathbf{7}$ & $\mathbf{8}$ & $\mathbf{9}$ & $\mathbf{1 0}$ \\
Total Revenue from sale of AJF & 0 & 117.9 & 117.9 & 117.9 & 117.93 & 117.9 & 117.9 & 117.93 & 117.93 & 117.9 & 117.9 \\
Total Revenue from emissions benefit & 0 & 5.90 & 5.90 & 5.90 & 5.90 & 5.90 & 5.90 & 5.90 & 5.90 & 5.90 & 5.90 \\
Total COP of AJF & 0 & 101.8 & 101.8 & 101.8 & 101.81 & 101.8 & 101.8 & 101.81 & 101.81 & 101.8 & 101.8 \\
EBITDA & 0 & 22.02 & 22.02 & 22.02 & 22.02 & 22.02 & 22.02 & 22.02 & 22.02 & 22.02 & 22.02 \\
Discount Factor & 1.00 & 1.07 & 1.14 & 1.23 & 1.31 & 1.40 & 1.50 & 1.61 & 1.72 & 1.84 & 1.97 \\
Discounted EBITDA & 0.00 & 20.58 & 19.23 & 17.97 & 16.80 & 15.70 & 14.67 & 13.71 & 12.82 & 11.98 & 11.19 \\
Terminal Value & & & & & & & & & & & \\
Upfront Capital Cost & -947.30 & & & & & & & & & &
\end{tabular}

\section{ASSUMPTIONS:}

Algae oil content $=25 \%$

System productivity $=13.2 \mathrm{~g} / \mathrm{m} 2 /$ day

Total pond size $=4050$ ha

COP of AJF $=\$ 2.59 / \mathrm{L}$

Total production $=39.31 \mathrm{ML} / \mathrm{Y}$

Total COP per year $=(39,310,000 \mathrm{~L} / \mathrm{Y}) \times(\$ 2.59 / \mathrm{L})=\$ 101,812,900$

Total Revenue from Sale of AJF per year $=(39,310,000 \mathrm{~L} / \mathrm{Y}) \times(\$ 3.00 / \mathrm{L})=\$ 117,930,000$

Hypothetical Jetfuel price $=\$ 3 / L(\$ 476.28 / \mathrm{bb})$

Emissions price $=\$ 60 / \mathrm{tCO} 2 \mathrm{e}$

Energy Density of Bio-SPK $=43.5 \mathrm{MJ} / \mathrm{kg}$

Algal oil denisty $=920 \mathrm{~kg} / \mathrm{m} 3$

Total production per year by energy $(\mathrm{MJ})=(39,310,000 \mathrm{~L}) \times(920 \mathrm{~kg} / \mathrm{m3}) \times(\mathrm{m} 3 / 1000 \mathrm{I}) \times(43.5 \mathrm{MJ} / \mathrm{kg})=1,573,186,200 \mathrm{MJ}$

Life-Cylce Emissions intensity of AJF $=25 \mathrm{gCO} 2 \mathrm{e} / \mathrm{MJ}$

Life-Cycle Emissions intensity of PJF $=87.5 \mathrm{gCO} 2 \mathrm{e} / \mathrm{MJ}$

Total Life-Cycle Emission per year from AJF $=(1,573,186,200 \mathrm{MJ}) \times(25 \mathrm{gCO} 2 \mathrm{e} / \mathrm{MJ})=39,329,655,000 \mathrm{MJ}$

Total Life-Cycle Emission per year from PJF $=(1,573,186,200 \mathrm{MJ}) \times(87.5 \mathrm{gCO} e / \mathrm{MJ})=137,653,792,500 \mathrm{gCO} e$

Total Life-Cycle Emission benefit per year $=(137,653,792,500)-(39,329,655,000)=98,324,137,500$ or $98,324.14$ tCO2

Total Monetized Life-Cycle Emission Benefit per year $=(98,324.14 \mathrm{tCO} 2) \times(\$ 60 / \mathrm{tCO})=\$ 5,899,448.25$

$\mathrm{WACC}=7 \%$

Total UCC $=\$ 947.3 \mathrm{M}$ 


\section{Calculations for Changing JFP \& Carbon Tax - @ \$3/1 \& \$120/tCO2e}

\begin{tabular}{lccccccccccc}
\multicolumn{1}{r}{$\quad$ Source of Cash Flow (SM) } & $\mathbf{0}$ & $\mathbf{1}$ & $\mathbf{2}$ & $\mathbf{3}$ & $\mathbf{4}$ & $\mathbf{5}$ & $\mathbf{6}$ & $\mathbf{7}$ & $\mathbf{8}$ & $\mathbf{9}$ & $\mathbf{1 0}$ \\
Total Revenue from sale of AJF & 0 & 117.9 & 117.9 & 117.9 & 117.93 & 117.9 & 117.9 & 117.93 & 117.93 & 117.9 & 117.9 \\
Total Revenue from emissions benefit & 0 & 11.98 & 11.98 & 11.98 & 11.98 & 11.98 & 11.98 & 11.98 & 11.98 & 11.98 & 11.98 \\
Total COP of AJF & 0 & 101.8 & 101.8 & 101.8 & 101.81 & 101.8 & 101.8 & 101.81 & 101.81 & 101.8 & 101.8 \\
EBITDA & 0 & 28.10 & 28.10 & 28.10 & 28.10 & 28.10 & 28.10 & 28.10 & 28.10 & 28.10 & 28.10 \\
Discount Factor & 1.00 & 1.07 & 1.14 & 1.23 & 1.31 & 1.40 & 1.50 & 1.61 & 1.72 & 1.84 & 1.97 \\
Discounted EBITDA & 0.00 & 26.26 & 24.54 & 22.94 & 21.44 & 20.03 & 18.72 & 17.50 & 16.35 & 15.28 & 14.28 \\
Terminal Value & & & & & & & & &
\end{tabular}

Upfront Capital Cost $\quad-947.30$

NPV $\quad-\mathbf{3 4 8 . 5 1}$

\section{ASSUMPTIONS:}

Algae oil content $=25 \%$

System productivity $=13.2 \mathrm{~g} / \mathrm{m} 2 /$ day

Total pond size $=4050$ ha

COP of AJF $=\$ 2.59 / \mathrm{L}$

Total production $=39.31 \mathrm{ML} / \mathrm{Y}$

Total COP per year $=(39,310,000 \mathrm{~L} / \mathrm{Y}) \times(\$ 2.59 / \mathrm{L})=\$ 101,812,900$

Total Revenue from Sale of AJF per year $=(39,310,000 \mathrm{~L} / \mathrm{Y}) \times(\$ 3.00 / \mathrm{L})=\$ 117,930,000$

Hypothetical Jetfuel price $=\$ 3 / \mathrm{L}(\$ 476.28 / \mathrm{bbl})$

Emissions price $=\$ 120 / \mathrm{tCO} 2 \mathrm{e}$

Energy Density of Bio-SPK $=43.5 \mathrm{MJ} / \mathrm{kg}$

Algal oil denisty $=920 \mathrm{~kg} / \mathrm{m} 3$

Total production per year by energy $(\mathrm{MJ})=(39,310,000 \mathrm{~L}) \times(920 \mathrm{~kg} / \mathrm{m3}) \times(\mathrm{m} 3 / 1000 \mathrm{l}) \times(43.5 \mathrm{MJ} / \mathrm{kg})=1,573,186,200 \mathrm{MJ}$

Life-Cylce Emissions intensity of AJF $=25 \mathrm{gCO} e / \mathrm{MJ}$

Life-Cycle Emissions intensity of PJF $=87.5 \mathrm{gCO} 2 \mathrm{e} / \mathrm{MJ}$

Total Life-Cycle Emission per year from AJF $=(1,573,186,200 \mathrm{MJ}) \times(25 \mathrm{gCO} e / \mathrm{MJ})=39,329,655,000 \mathrm{MJ}$

Total Life-Cycle Emission per year from PJF $=(1,573,186,200 \mathrm{MJ}) \times(87.5 \mathrm{gCO} e / \mathrm{MJ})=137,653,792,500 \mathrm{gCO} e$

Total Life-Cycle Emission benefit per year $=(137,653,792,500)-(39,329,655,000)=98,324,137,500$ or $98,324.14$ tCO2

Total Monetized Life-Cycle Emission Benefit per year $=(98,324.14 \mathrm{tCO} 2) \times(\$ 120 / \mathrm{tCO})=\$ 11,978,896.50$

WACC $=7 \%$

Total UCC $=\$ 947.3 \mathrm{M}$ 


\section{Calculations for Changing JFP \& Carbon Tax - @ \$3/1 \& \$200/tCO2e}

\begin{tabular}{|c|c|c|c|c|c|c|c|c|c|c|c|}
\hline \multirow[b]{2}{*}{ Source of Cash Flow (\$M) } & \multicolumn{9}{|c|}{ Year (N) } & \multirow[b]{2}{*}{9} & \multirow[b]{2}{*}{10} \\
\hline & 0 & 1 & 2 & 3 & 4 & 5 & 6 & 7 & 8 & & \\
\hline Total Revenue from emissions benefit & 0 & 19.66 & 19.66 & 19.66 & 19.66 & 19.66 & 19.66 & 19.66 & 19.66 & 19.66 & 19.66 \\
\hline EBITDA & 0 & 35.78 & 35.78 & 35.78 & 35.78 & 35.78 & 35.78 & 35.78 & 35.78 & 35.78 & 35.78 \\
\hline Discount Factor & 1.00 & 1.07 & 1.14 & 1.23 & 1.31 & 1.40 & 1.50 & 1.61 & 1.72 & 1.84 & 1.97 \\
\hline Discounted EBITDA & 0.00 & 33.44 & 31.25 & 29.21 & 27.30 & 25.51 & 23.84 & 22.28 & 20.82 & 19.46 & 18.19 \\
\hline
\end{tabular}

Upfront Capital Cost $\quad-947.30$

$-184.85$

ASSUMPTIONS:

Algae oil content $=25 \%$

System productivity $=13.2 \mathrm{~g} / \mathrm{m} 2 /$ day

Total pond size $=4050$ ha

COP of AJF $=\$ 2.59 / \mathrm{L}$

Total production $=39.31 \mathrm{ML} / \mathrm{Y}$

Total COP per year $=(39,310,000 \mathrm{~L} / \mathrm{Y}) \times(\$ 2.59 / \mathrm{L})=\$ 101,812,900$

Total Revenue from Sale of AJF per year $=(39,310,000 \mathrm{~L} / \mathrm{Y}) \times(\$ 3.00 / \mathrm{L})=\$ 117,930,000$

Hypothetical Jetfuel price $=\$ 3 / L(\$ 476.28 / \mathrm{bbl})$

Emissions price $=\$ 200 / \mathrm{tCO} \mathrm{e}$

Energy Density of Bio-SPK $=43.5 \mathrm{MJ} / \mathrm{kg}$

Algal oil denisty $=920 \mathrm{~kg} / \mathrm{m} 3$

Total production per year by energy $(\mathrm{MJ})=(39,310,000 \mathrm{~L}) \times(920 \mathrm{~kg} / \mathrm{m3}) \times(\mathrm{m3} / 1000 \mathrm{I}) \times(43.5 \mathrm{MJ} / \mathrm{kg})=1,573,186,200 \mathrm{MJ}$

Life-Cylce Emissions intensity of AJF $=25 \mathrm{gCO} 2 \mathrm{e} / \mathrm{MJ}$

Life-Cycle Emissions intensity of PJF $=87.5 \mathrm{gCO} 2 \mathrm{e} / \mathrm{MJ}$

Total Life-Cycle Emission per year from AJF $=(1,573,186,200 \mathrm{MJ}) \times(25 \mathrm{gCO} e / \mathrm{MJ})=39,329,655,000 \mathrm{MJ}$

Total Life-Cycle Emission per year from PJF $=(1,573,186,200 \mathrm{MJ}) \times(87.5 \mathrm{gCO} 2 \mathrm{e} / \mathrm{MJ})=137,653,792,500 \mathrm{gCO} \mathrm{e}$

Total Life-Cycle Emission benefit per year $=(137,653,792,500)-(39,329,655,000)=98,324,137,500$ or $98,324.14$ tCO2

Total Monetized Life-Cycle Emission Benefit per year $=(98,324.14 \mathrm{tCO}) \times(\$ 200 / \mathrm{tCO})=\$ 19,664,827.50$

WACC $=7 \%$

Total UCC $=\$ 947.3 \mathrm{M}$ 


\section{Calculations for Changing JFP \& Carbon Tax - @ \$3/1 \& \$400/tCO2e}

\begin{tabular}{lccccccccccc}
\multicolumn{1}{r}{ Source of Cash Flow (\$M) } & $\mathbf{0}$ & $\mathbf{1}$ & $\mathbf{2}$ & $\mathbf{3}$ & $\mathbf{4}$ & $\mathbf{5}$ & $\mathbf{6}$ & $\mathbf{7}$ & $\mathbf{8}$ & $\mathbf{9}$ & $\mathbf{1 0}$ \\
Total Revenue from sale of AJF & 0 & 117.9 & 117.9 & 117.9 & 117.93 & 117.9 & 117.9 & 117.93 & 117.93 & 117.9 & 117.9 \\
Total Revenue from emissions benefit & 0 & 39.33 & 39.33 & 39.33 & 39.33 & 39.33 & 39.33 & 39.33 & 39.33 & 39.33 & 39.33 \\
Total COP of AJF & 0 & 101.8 & 101.8 & 101.8 & 101.81 & 101.8 & 101.8 & 101.81 & 101.81 & 101.8 & 101.8 \\
EBITDA & 0 & 55.45 & 55.45 & 55.45 & 55.45 & 55.45 & 55.45 & 55.45 & 55.45 & 55.45 & 55.45 \\
Discount Factor & 1.00 & 1.07 & 1.14 & 1.23 & 1.31 & 1.40 & 1.50 & 1.61 & 1.72 & 1.84 & 1.97 \\
Discounted EBITDA & 0.00 & 51.82 & 48.43 & 45.26 & 42.30 & 39.54 & 36.95 & 34.53 & 32.27 & 30.16 & 28.19 \\
Terminal Value & & & & & & & & & & & \\
Upfront Capital Cost & -947.30 & & & & & & & & & &
\end{tabular}

\section{ASSUMPTIONS}

Algae oil content $=25 \%$

System productivity $=13.2 \mathrm{~g} / \mathrm{m} 2 /$ day

Total pond size $=4050$ ha

COP of AJF $=\$ 2.59 / \mathrm{L}$

Total production $=39.31 \mathrm{ML} / \mathrm{Y}$

Total COP per year $=(39,310,000 \mathrm{~L} / \mathrm{Y}) \times(\$ 2.59 / \mathrm{L})=\$ 101,812,900$

Total Revenue from Sale of AJF per year $=(39,310,000 \mathrm{~L} / \mathrm{Y}) \times(\$ 3.00 / \mathrm{L})=\$ 117,930,000$

Hypothetical Jetfuel price $=\$ 3 / \mathrm{L}(\$ 476.28 / \mathrm{bbl})$

Emissions price $=\$ 400 / \mathrm{tCO} 2 \mathrm{e}$

Energy Density of Bio-SPK $=43.5 \mathrm{MJ} / \mathrm{kg}$

Algal oil denisty $=920 \mathrm{~kg} / \mathrm{m} 3$

Total production per year by energy $(\mathrm{MJ})=(39,310,000 \mathrm{~L}) \times(920 \mathrm{~kg} / \mathrm{m3}) \times(\mathrm{m3} / 1000 \mathrm{I}) \times(43.5 \mathrm{MJ} / \mathrm{kg})=1,573,186,200 \mathrm{MJ}$

Life-Cylce Emissions intensity of AJF $=25 \mathrm{gCO} 2 \mathrm{e} / \mathrm{MJ}$

Life-Cycle Emissions intensity of PJF $=87.5 \mathrm{gCO} 2 \mathrm{e} / \mathrm{MJ}$

Total Life-Cycle Emission per year from AJF $=(1,573,186,200 \mathrm{MJ}) \times(25 \mathrm{gCO} 2 \mathrm{e} / \mathrm{MJ})=39,329,655,000 \mathrm{MJ}$

Total Life-Cycle Emission per year from PJF $=(1,573,186,200 \mathrm{MJ}) \times(87.5 \mathrm{gCO} \mathrm{e} / \mathrm{MJ})=137,653,792,500 \mathrm{gCO} \mathrm{e}$

Total Life-Cycle Emission benefit per year $=(137,653,792,500)-(39,329,655,000)=98,324,137,500$ or $98,324.14$ tCO2

Total Monetized Life-Cycle Emission Benefit per year $=(98,324.14 \mathrm{tCO}) \times(\$ 400 / \mathrm{tCO} 2)=\$ 39,329,656$

WACC $=7 \%$

Total UCC $=\$ 947.3 \mathrm{M}$ 


\section{Calculations for Changing JFP \& Carbon Tax - @ \$3/1 \& \$800/tCO2e}

\begin{tabular}{|c|c|c|c|c|c|c|c|c|c|c|c|}
\hline \multirow[b]{2}{*}{ Source of Cash Flow (\$M) } & \multicolumn{9}{|c|}{ Year (N) } & \multirow[b]{2}{*}{9} & \multirow[b]{2}{*}{10} \\
\hline & $\mathbf{0}$ & 1 & 2 & 3 & 4 & 5 & 6 & 7 & 8 & & \\
\hline Total Revenue from emissions benefit & 0 & 78.66 & 78.66 & 78.66 & 78.66 & 78.66 & 78.66 & 78.66 & 78.66 & 78.66 & 78.66 \\
\hline EBITDA & 0 & 94.78 & 94.78 & 94.78 & 94.78 & 94.78 & 94.78 & 94.78 & 94.78 & 94.78 & 94.78 \\
\hline Discount Factor & 1.00 & 1.07 & 1.14 & 1.23 & 1.31 & 1.40 & 1.50 & 1.61 & 1.72 & 1.84 & 1.97 \\
\hline Discounted EBITDA & 0.00 & 88.58 & 82.78 & 77.37 & 72.31 & 67.58 & 63.16 & 59.02 & 55.16 & 51.55 & 48.18 \\
\hline Upfront Capital Cost & -947.30 & & & & & & & & & & \\
\hline NPV & 1072.40 & & & & & & & & & & \\
\hline
\end{tabular}

\section{ASSUMPTIONS:}

Algae oil content $=25 \%$

System productivity $=13.2 \mathrm{~g} / \mathrm{m} 2 /$ day

Total pond size $=4050$ ha

COP of AJF $=\$ 2.59 / \mathrm{L}$

Total production $=39.31 \mathrm{ML} / \mathrm{Y}$

Total COP per year $=(39,310,000 \mathrm{~L} / \mathrm{Y}) \times(\$ 2.59 / \mathrm{L})=\$ 101,812,900$

Total Revenue from Sale of AJF per year $=(39,310,000 \mathrm{~L} / \mathrm{Y}) \times(\$ 3.00 / \mathrm{L})=\$ 117,930,000$

Hypothetical Jetfuel price $=\$ 3 / \mathrm{L}(\$ 476.28 / \mathrm{bbl})$

Emissions price $=\$ 800 / \mathrm{tCO} 2 \mathrm{e}$

Energy Density of Bio-SPK $=43.5 \mathrm{MJ} / \mathrm{kg}$

Algal oil denisty $=920 \mathrm{~kg} / \mathrm{m} 3$

Total production per year by energy $(\mathrm{MJ})=(39,310,000 \mathrm{~L}) \times(920 \mathrm{~kg} / \mathrm{m3}) \times(\mathrm{m} 3 / 1000 \mathrm{I}) \times(43.5 \mathrm{MJ} / \mathrm{kg})=1,573,186,200 \mathrm{MJ}$

Life-Cylce Emissions intensity of AJF $=25 \mathrm{gCO} 2 \mathrm{e} / \mathrm{MJ}$

Life-Cycle Emissions intensity of PJF $=87.5 \mathrm{gCO} e / \mathrm{MJ}$

Total Life-Cycle Emission per year from AJF $=(1,573,186,200 \mathrm{MJ}) \times(25 \mathrm{gCO} e / \mathrm{MJ})=39,329,655,000 \mathrm{MJ}$

Total Life-Cycle Emission per year from PJF $=(1,573,186,200 \mathrm{MJ}) \times(87.5 \mathrm{gCO} 2 \mathrm{e} / \mathrm{MJ})=137,653,792,500 \mathrm{gCO} \mathrm{e}$

Total Life-Cycle Emission benefit per year $=(137,653,792,500)-(39,329,655,000)=98,324,137,500$ or $98,324.14$ tCO2

Total Monetized Life-Cycle Emission Benefit per year $=(98,324.14 \mathrm{tCO}) \times(\$ 800 / \mathrm{tCO})=\$ 78,659,312$

WACC $=7 \%$

Total UCC $=\$ 947.3 \mathrm{M}$ 


\section{Calculations for Changing JFP \& Carbon Tax - @ \$3/1 \& \$2000/tC02e}

\begin{tabular}{|c|c|c|c|c|c|c|c|c|c|c|c|}
\hline \multirow[b]{2}{*}{ Source of Cash Flow (\$M) } & \multicolumn{9}{|c|}{ Year (N) } & \multirow[b]{2}{*}{9} & \multirow[b]{2}{*}{10} \\
\hline & 0 & 1 & 2 & 3 & 4 & 5 & 6 & 7 & 8 & & \\
\hline Total Revenue from emissions benefit & 0 & 196.65 & 196.65 & 196.65 & 196.65 & 196.65 & 196.65 & 196.65 & 196.65 & 196.65 & 196.65 \\
\hline EBITDA & 0 & 212.77 & 212.77 & 212.77 & 212.77 & 212.77 & 212.77 & 212.77 & 212.77 & 212.77 & 212.77 \\
\hline Discount Factor & 1.00 & 1.07 & 1.14 & 1.23 & 1.31 & 1.40 & 1.50 & 1.61 & 1.72 & 1.84 & 1.97 \\
\hline Discounted EBITDA & 0.00 & 198.85 & 185.84 & 173.68 & 162.32 & 151.70 & 141.78 & 132.50 & 123.83 & 115.73 & 108.16 \\
\hline Upfront Capital Cost & -947.30 & & & & & & & & & & \\
\hline NPV & 3586.68 & & & & & & & & & & \\
\hline
\end{tabular}

\section{ASSUMPTIONS:}

Algae oil content $=25 \%$

System productivity $=13.2 \mathrm{~g} / \mathrm{m} 2 /$ day

Total pond size $=4050$ ha

COP of AJF $=\$ 2.59 / \mathrm{L}$

Total production $=39.31 \mathrm{ML} / \mathrm{Y}$

Total COP per year $=(39,310,000 \mathrm{~L} / \mathrm{Y}) \times(\$ 2.59 / \mathrm{L})=\$ 101,812,900$

Total Revenue from Sale of AJF per year $=(39,310,000 \mathrm{~L} / \mathrm{Y}) \times(\$ 3.00 / \mathrm{L})=\$ 117,930,000$

Hypothetical Jetfuel price $=\$ 3 / \mathrm{L}(\$ 476.28 / \mathrm{bbl})$

Emissions price $=\$ 2000 / \mathrm{tCO} 2 \mathrm{e}$

Energy Density of Bio-SPK $=43.5 \mathrm{MJ} / \mathrm{kg}$

Algal oil denisty $=920 \mathrm{~kg} / \mathrm{m} 3$

Total production per year by energy $(\mathrm{MJ})=(39,310,000 \mathrm{~L}) \times(920 \mathrm{~kg} / \mathrm{m3}) \times(\mathrm{m3} / 1000 \mathrm{I}) \times(43.5 \mathrm{MJ} / \mathrm{kg})=1,573,186,200 \mathrm{MJ}$

Life-Cylce Emissions intensity of AJF $=25 \mathrm{gCO} 2 \mathrm{e} / \mathrm{MJ}$

Life-Cycle Emissions intensity of PJF $=87.5 \mathrm{gCO} 2 \mathrm{e} / \mathrm{MJ}$

Total Life-Cycle Emission per year from AJF $=(1,573,186,200 \mathrm{MJ}) \times(25 \mathrm{gCO} 2 \mathrm{e} / \mathrm{MJ})=39,329,655,000 \mathrm{MJ}$

Total Life-Cycle Emission per year from PJF $=(1,573,186,200 \mathrm{MJ}) \times(87.5 \mathrm{gCO} \mathrm{e} / \mathrm{MJ})=137,653,792,500 \mathrm{gCO} 2 \mathrm{e}$

Total Life-Cycle Emission benefit per year $=(137,653,792,500)-(39,329,655,000)=98,324,137,500$ or $98,324.14$ tCO2

Total Monetized Life-Cycle Emission Benefit per year $=(98,324.14 \mathrm{tCO}) \times(\$ 2000 / \mathrm{tCO})=\$ 196,648,280$

WACC $=7 \%$

Total UCC $=\$ 947.3 \mathrm{M}$ 


\section{APPENDIX E}

\section{Calculations for Algal Oil Content \& Productivity - @ 50\% \& 40 g/m2/day (Medium Growth)}

\begin{tabular}{|c|c|c|c|c|c|c|c|c|c|c|c|}
\hline Source of Cash Flow (\$M) & \multicolumn{10}{|c|}{ Year (N) } & 10 \\
\hline Total Revenue from emissions benefit & 0 & 21.8 & 21.8 & 21.8 & 21.8 & 21.8 & 21.8 & 21.8 & 21.8 & 21.8 & 21.8 \\
\hline EBITDA & 0 & -155.4 & -155.4 & -155 & -155 & -155 & -155 & -155.4 & -155.4 & -155 & -155.4 \\
\hline Discount Factor & 1.00 & 1.07 & 1.14 & 1.23 & 1.31 & 1.40 & 1.50 & 1.61 & 1.72 & 1.84 & 1.97 \\
\hline Discounted EBITDA & 0.00 & -145.26 & -135.76 & -126.88 & -118.58 & -110.82 & -103.57 & -96.79 & -90.46 & -84.54 & -79.01 \\
\hline Upfront Capital Cost & -947.30 & & & & & & & & & & \\
\hline NPV & -4259.40 & & & & & & & & & & \\
\hline
\end{tabular}

\section{ASSUMPTIONS:}

Algae oil content $=50 \%$

System productivity $=40 \mathrm{~g} / \mathrm{m} 2 /$ day

Total pond size $=4050$ ha

Total algal oil produced $=[(40 \mathrm{~g} / \mathrm{m} 2 /$ day $) \times(10,000 \mathrm{~m} 2 / \mathrm{ha}) \times(4050 \mathrm{ha}) \times(0.50 \mathrm{gAlgal}-$ oil $/ \mathrm{gAlgae}) \times(330 \mathrm{days} / \mathrm{year})] /[1000 \mathrm{~g} / \mathrm{kg}]=267,300,000 \mathrm{Kg} / \mathrm{Y}$

Algal oil denisty $=920 \mathrm{~kg} / \mathrm{m} 3$

Total oil produced $=[(267,300,000 \mathrm{~kg}) /(920 \mathrm{~kg} / \mathrm{m3})] \times(1000 \mathrm{~L} / \mathrm{m3})=290,543,478.30 \mathrm{~L} / \mathrm{Y}$

COP of AJF $=(\$ 1.11 / \mathrm{L})+(\$ 0.34 / \mathrm{L})=\$ 1.45 / \mathrm{L}$

Total COP $=(290,543.478 .30 \mathrm{~L} / \mathrm{Y}) \times(\$ 1.45 / \mathrm{L})=\$ 421,288,043.50$

Total Revenue from Sale of AJF $=290,543.478 .30 \mathrm{~L} / \mathrm{Y}) \times(\$ 0.84 / \mathrm{L})=\$ 244,056,521,70$

Average price of Jetfuel for $2013=\$ 132.80 / \mathrm{bbl}$ or $\$ 0.84 / \mathrm{L}$

Emissions price $=\$ 30 / \mathrm{tCO} 2 \mathrm{e}$

Energy Density of Bio-SPK $=43.5 \mathrm{MJ} / \mathrm{kg}$

Total production per year by energy $(\mathrm{MJ})=(267,300,000 \mathrm{Kg} / \mathrm{Y}) \times(43.5 \mathrm{MJ} / \mathrm{kg})=11,627,550,000 \mathrm{MJ}$

Life-Cylce Emission intensity of AJF $=25 \mathrm{gCO} e / \mathrm{MJ}$

Total Life-Cycle Emission per year from AJF $=(11,627,550,000 \mathrm{MJ}) \times(25 \mathrm{gCO} e / \mathrm{MJ})=290,688,750,000 \mathrm{gCO} e$

Life-Cycle Emissions intensity of PJF $=87.5 \mathrm{gCO} e / \mathrm{MJ}$

Total Life-Cycle Emission per year from PJF $=(11,627,550,000 \mathrm{MJ}) \times(87.5 \mathrm{gCO} 2 \mathrm{e} / \mathrm{MJ})=1,017,410,625,000 \mathrm{gCO} e$

Total Life-Cycle Emission benefit per year $=(1,017,410,625,000)-(290,688,750,000)=726,721,875,000$ or $726,721.88$ tCO2

Total Monetized Life-Cycle Emission Benefit per year $=(726,721.88 \mathrm{tCO} 2) \times(\$ 30 / \mathrm{tCO})=\$ 21,801,656.25$

WACC $=7 \%$

Total UCC $=\$ 947.3 \mathrm{M}$ 


\section{Calculations for Algal Oil Content \& Productivity - @ 60\% \& 60 g/m2/day}

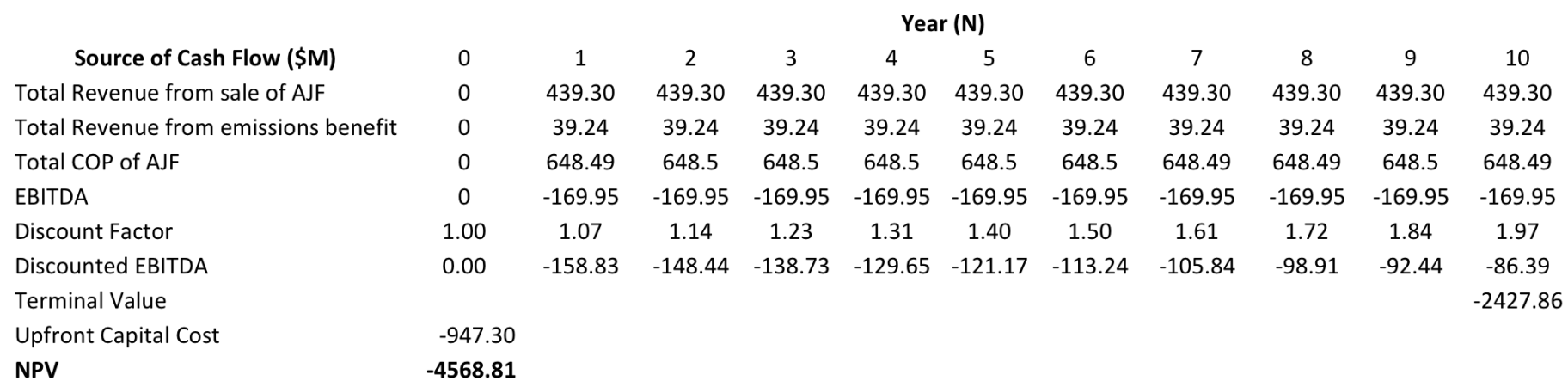

\section{ASSUMPTIONS:}

Algae oil content $=60 \%$

System productivity $=60 \mathrm{~g} / \mathrm{m} 2 /$ day

Total pond size $=4050$ ha

Total algal oil produced $=[(60 \mathrm{~g} / \mathrm{m} 2 /$ day $) \times(10,000 \mathrm{~m} 2 / \mathrm{ha}) \times(4050 \mathrm{ha}) \times(0.60 \mathrm{gAlgal}-\mathrm{oi} / \mathrm{gAlgae}) \times(330 \mathrm{days} / \mathrm{year})] /[1000 \mathrm{~g} / \mathrm{kg}]=481,140,000 \mathrm{Kg} / \mathrm{Y}$

Algal oil denisty $=920 \mathrm{~kg} / \mathrm{m} 3$

Total oil produced $=[(481,140,000 \mathrm{~kg}) /(920 \mathrm{~kg} / \mathrm{m3})] \times(1000 \mathrm{~L} / \mathrm{m3})=522,978,260.90 \mathrm{~L} / \mathrm{Y}$

COP of AJF $=(\$ 0.90 / \mathrm{L})+(\$ 0.34 / \mathrm{L})=\$ 1.24 / \mathrm{L}$

Total COP $=(522,978,260.90 \mathrm{~L} / \mathrm{Y}) \times(\$ 1.24 / \mathrm{L})=\$ 648,493,043.50$

Total Revenue from Sale of AJF $=489,460,834.20 \mathrm{~L} / \mathrm{Y}) \times(\$ 0.84 / \mathrm{L})=\$ 439,301,739.10$

Average price of Jetfuel for $2013=\$ 132.80 / \mathrm{bbl}$ or $\$ 0.84 / \mathrm{L}$

Emissions price $=\$ 30 / \mathrm{tCO} 2 \mathrm{e}$

Energy Density of Bio-SPK $=43.5 \mathrm{MJ} / \mathrm{kg}$

Total production per year by energy $(\mathrm{MJ})=(481,140,000 \mathrm{Kg} / \mathrm{Y}) \times(43.5 \mathrm{MJ} / \mathrm{kg})=2,092,9590,000 \mathrm{MJ}$

Life-Cylce Emission intensity of AJF $=25 \mathrm{gCO} e / \mathrm{MJ}$

Total Life-Cycle Emission per year from AJF $=(2,092,9590,000 \mathrm{MJ}) \times(25 \mathrm{gCO} e / \mathrm{MJ})=523,239,750,000 \mathrm{gCO} e$

Life-Cycle Emissions intensity of PJF $=87.5 \mathrm{gCO} e / \mathrm{MJ}$

Total Life-Cycle Emission per year from PJF $=(2,092,9590,000 \mathrm{MJ}) \times(87.5 \mathrm{gCO} \mathrm{e} / \mathrm{MJ})=1,831,339,125,000 \mathrm{gCO} \mathrm{e}$

Total Life-Cycle Emission benefit per year $=(1,831,339,125,000)-(523,239,750,000)=1,308,099,375,000$ or $1,308,099.38$ tCO2

Total Monetized Life-Cycle Emission Benefit per year $=(1,308,099.38 \mathrm{tCO}) \times(\$ 30 / \mathrm{tCO})=\$ 39,242,981.25$

WACC $=7 \%$

Total UCC $=\$ 947.3 \mathrm{M}$ 


\section{APPENDIX F}

\section{Calculations for Changing Algal Oil Content, Productivity \& JFP - @ 50\%, 40 g/m2/day \& $\$ 1 / 1$}

\begin{tabular}{|c|c|c|c|c|c|c|c|c|c|c|c|}
\hline Source of Cash Flow (\$M) & \multicolumn{11}{|c|}{ Year (N) } \\
\hline Total Revenue from emissions benefit & 0 & 21.8 & 21.8 & 21.8 & 21.8 & 21.8 & 21.8 & 21.8 & 21.8 & 21.8 & 21.8 \\
\hline EBITDA & 0 & -109 & -81.95 & -81.95 & -81.95 & -81.95 & -82 & -82 & -81.95 & -81.95 & -81.95 \\
\hline Discount Factor & 1.00 & 1.07 & 1.14 & 1.23 & 1.31 & 1.40 & 1.50 & 1.61 & 1.72 & 1.84 & 1.97 \\
\hline Discounted EBITDA & 0.00 & -101.82 & -71.58 & -66.90 & -62.52 & -58.43 & -54.61 & -51.03 & -47.70 & -44.58 & -41.66 \\
\hline Upfront Capital Cost & -947.30 & & & & & & & & & & \\
\hline NPV & -2718.83 & & & & & & & & & & \\
\hline
\end{tabular}

\section{ASSUMPTIONS:}

Algae oil content $=50 \%$

System productivity $=40 \mathrm{~g} / \mathrm{m} 2 /$ day

Total pond size $=4050$ ha

Total algal oil produced $=[(40 \mathrm{~g} / \mathrm{m} 2 /$ day $) \times(10,000 \mathrm{m2} / \mathrm{ha}) \times(4050 \mathrm{ha}) \times(0.50 \mathrm{gAlgal}-\mathrm{oi} / \mathrm{gA}$ gae $) \times(330$ days $/$ year $)] /[1000 \mathrm{~g} / \mathrm{kg}]=267,300,000 \mathrm{Kg} / \mathrm{Y}$ Algal oil denisty $=920 \mathrm{~kg} / \mathrm{m} 3$

Total oil produced $=[(267,300,000 \mathrm{~kg}) /(920 \mathrm{~kg} / \mathrm{m3})] \times(1000 \mathrm{~L} / \mathrm{m3})=290,543,478.30 \mathrm{~L} / \mathrm{Y}$

COP of AJF $=(\$ 1.11 / \mathrm{L})+(\$ 0.34 / \mathrm{L})=\$ 1.45 / \mathrm{L}$

Total COP $=(290,543.478 .30 \mathrm{~L} / \mathrm{Y}) \times(\$ 1.45 / \mathrm{L})=\$ 421,288,043.50$

Total Revenue from Sale of AJF $=290,543.478 .30 \mathrm{~L} / \mathrm{Y}) \times(\$ 1.00 / \mathrm{L})=\$ 290,543.478 .30$

Hypothetical Jetfuel price $=\$ 1 / \mathrm{L}(\$ 158.76 / \mathrm{bbl})$

Emissions price $=\$ 30 / \mathrm{tCO} 2 \mathrm{e}$

Energy Density of Bio-SPK $=43.5 \mathrm{MJ} / \mathrm{kg}$

Total production per year by energy $(\mathrm{MJ})=(267,300,000 \mathrm{Kg} / \mathrm{Y}) \times(43.5 \mathrm{MJ} / \mathrm{kg})=11,627,550,000 \mathrm{MJ}$

Life-Cylce Emission intensity of AJF $=25 \mathrm{gCO} e / \mathrm{MJ}$

*Boeing 2009

Total Life-Cycle Emission per year from AJF $=(11,627,550,000 \mathrm{MJ}) \times(25 \mathrm{gCO} e / \mathrm{MJ})=290,688,750,000 \mathrm{gCO} e$

Life-Cycle Emissions intensity of PJF $=87.5 \mathrm{gCO} e / \mathrm{MJ}$

Total Life-Cycle Emission per year from PJF $=(11,627,550,000 \mathrm{MJ}) \times(87.5 \mathrm{gCO} 2 \mathrm{e} / \mathrm{MJ})=1,017,410,625,000 \mathrm{gCO} e$

Total Life-Cycle Emission benefit per year $=(1,017,410,625,000)-(290,688,750,000)=726,721,875,000$ or $726,721.88$ tCO2

Total Monetized Life-Cycle Emission Benefit per year $=(726,721.88 \mathrm{tCO} 2) \times(\$ 30 / \mathrm{tCO} 2)=\$ 21,801,656.25$

WACC $=7 \%$

Total UCC $=\$ 947.3 \mathrm{M}$ 


\section{Calculations for Changing Algal Oil Content, Productivity \& JFP - @ 50\%, 40 g/m2/day \& $\$ 1.50 / 1$}

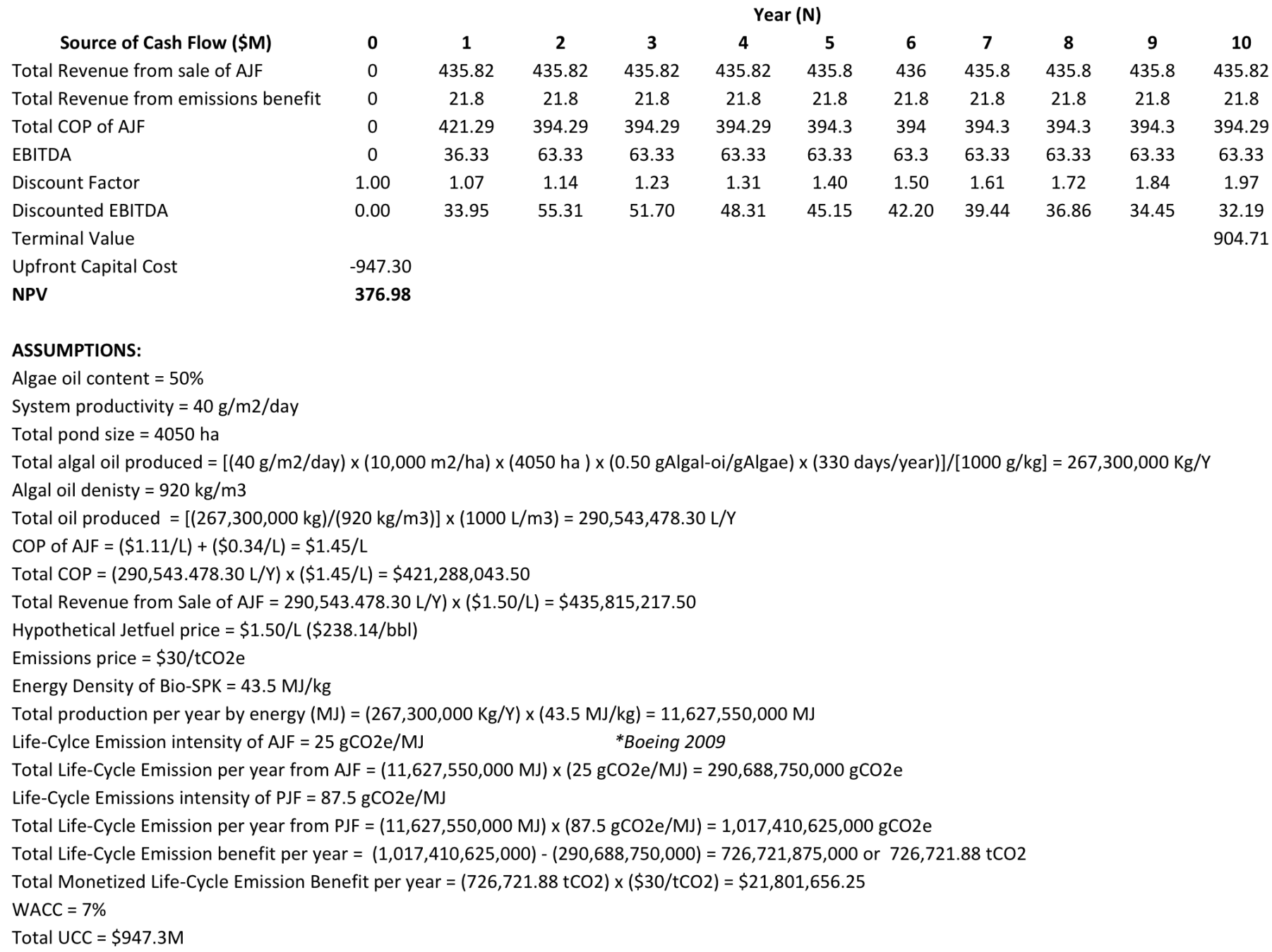




\section{Calculations for Changing Algal Oil Content, Productivity \& JFP - @ 50\%, 40 g/m2/day \& $\$ 3 / 1$}

\begin{tabular}{|c|c|c|c|c|c|c|c|c|c|c|c|}
\hline \multirow[b]{2}{*}{ Source of Cash Flow (\$M) } & \multicolumn{10}{|c|}{ Year (N) } & \multirow[b]{2}{*}{10} \\
\hline & $\mathbf{0}$ & 1 & 2 & 3 & 4 & 5 & 6 & 7 & 8 & 9 & \\
\hline Total Revenue from emissions benefit & 0 & 21.8 & 21.8 & 21.8 & 21.8 & 21.8 & 21.8 & 21.8 & 21.8 & 21.8 & 21.8 \\
\hline EBITDA & 0 & 472.14 & 499.14 & 499.14 & 499.14 & 499.1 & 499 & 499.1 & 499.1 & 499.1 & 499.14 \\
\hline Discount Factor & 1.00 & 1.07 & 1.14 & 1.23 & 1.31 & 1.40 & 1.50 & 1.61 & 1.72 & 1.84 & 1.97 \\
\hline Discounted EBITDA & 0.00 & 441.25 & 435.97 & 407.45 & 380.79 & 355.88 & 332.60 & 310.84 & 290.50 & 271.50 & 253.74 \\
\hline Upfront Capital Cost & -947.30 & & & & & & & & & & \\
\hline NPV & 9663.79 & & & & & & & & & & \\
\hline
\end{tabular}

\section{ASSUMPTIONS:}

Algae oil content $=50 \%$

System productivity $=40 \mathrm{~g} / \mathrm{m} 2 /$ day

Total pond size $=4050$ ha

Total algal oil produced $=[(40 \mathrm{~g} / \mathrm{m} 2 /$ day $) \times(10,000 \mathrm{~m} 2 / \mathrm{ha}) \times(4050 \mathrm{ha}) \times(0.50 \mathrm{gAlgal}-\mathrm{oi} / \mathrm{gAlgae}) \times(330 \mathrm{days} /$ year $)] /[1000 \mathrm{~g} / \mathrm{kg}]=267,300,000 \mathrm{Kg} / \mathrm{Y}$ Algal oil denisty $=920 \mathrm{~kg} / \mathrm{m} 3$

Total oil produced $=[(267,300,000 \mathrm{~kg}) /(920 \mathrm{~kg} / \mathrm{m3})] \times(1000 \mathrm{~L} / \mathrm{m3})=290,543,478.30 \mathrm{~L} / \mathrm{Y}$

COP of AJF $=(\$ 1.11 / \mathrm{L})+(\$ 0.34 / \mathrm{L})=\$ 1.45 / \mathrm{L}$

Total COP $=(290,543.478 .30 \mathrm{~L} / \mathrm{Y}) \times(\$ 1.45 / \mathrm{L})=\$ 421,288,043.50$

Total Revenue from Sale of AJF $=290,543.478 .30 \mathrm{~L} / \mathrm{Y}) \times(\$ 3 / \mathrm{L})=\$ 871,630,434.90$

Hypothetical Jetfuel price $=\$ 3 / \mathrm{L}(\$ 476.28 / \mathrm{bbl})$

Emissions price $=\$ 30 / \mathrm{tCO} 2 \mathrm{e}$

Energy Density of Bio-SPK $=43.5 \mathrm{MJ} / \mathrm{kg}$

Total production per year by energy $(\mathrm{MJ})=(267,300,000 \mathrm{Kg} / \mathrm{Y}) \times(43.5 \mathrm{MJ} / \mathrm{kg})=11,627,550,000 \mathrm{MJ}$

Life-Cylce Emission intensity of AJF $=25$ gCO2e/MJ

Total Life-Cycle Emission per year from AJF $=(11,627,550,000 \mathrm{MJ}) \times(25 \mathrm{gCO} e / \mathrm{MJ})=290,688,750,000 \mathrm{gCO} e$

Life-Cycle Emissions intensity of PJF $=87.5 \mathrm{gCO} 2 \mathrm{e} / \mathrm{MJ}$

Total Life-Cycle Emission per year from PJF $=(11,627,550,000 \mathrm{MJ}) \times(87.5 \mathrm{gCO} 2 \mathrm{e} / \mathrm{MJ})=1,017,410,625,000 \mathrm{gCO} \mathrm{e}$

Total Life-Cycle Emission benefit per year $=(1,017,410,625,000)-(290,688,750,000)=726,721,875,000$ or $726,721.88$ tCO2

Total Monetized Life-Cycle Emission Benefit per year $=(726,721.88 \mathrm{tCO}) \times(\$ 30 / \mathrm{tCO} 2)=\$ 21,801,656.25$

WACC $=7 \%$

Total UCC $=\$ 947.3 \mathrm{M}$ 


\section{Calculations for Changing Algal Oil Content, Productivity \& JFP - @ 60\%,60 g/m2/day \& $\$ 1 / 1$}

\begin{tabular}{|c|c|c|c|c|c|c|c|c|c|c|c|}
\hline Source of Cash Flow (\$M) & \multicolumn{11}{|c|}{ Year (N) } \\
\hline Total Revenue from emissions benefit & 0 & 39.24 & 39.24 & 39.24 & 39.24 & 39.24 & 39.2 & 39.24 & 39.24 & 39.24 & 39.24 \\
\hline EBITDA & 0 & -86.27 & -86.27 & -86.27 & -86.27 & -86.27 & -86.3 & -86.3 & -86.27 & -86.27 & -86.27 \\
\hline Discount Factor & 1.00 & 1.07 & 1.14 & 1.23 & 1.31 & 1.40 & 1.50 & 1.61 & 1.72 & 1.84 & 1.97 \\
\hline Discounted EBITDA & 0.00 & -80.63 & -75.35 & -70.42 & -65.81 & -61.51 & -57.49 & -53.72 & -50.21 & -46.93 & -43.86 \\
\hline Upfront Capital Cost & -947.30 & & & & & & & & & & \\
\hline NPV & -2785.65 & & & & & & & & & & \\
\hline
\end{tabular}

\section{ASSUMPTIONS:}

Algae oil content $=60 \%$

System productivity $=60 \mathrm{~g} / \mathrm{m} 2 /$ day

Total pond size $=4050$ ha

Total algal oil produced $=[(60 \mathrm{~g} / \mathrm{m} 2 /$ day $) \times(10,000 \mathrm{~m} 2 / \mathrm{ha}) \times(4050 \mathrm{ha}) \times(0.60 \mathrm{gAlgal}-\mathrm{oi} / \mathrm{gAlgae}) \times(330$ days $/$ year $)] /[1000 \mathrm{~g} / \mathrm{kg}]=481,140,000 \mathrm{Kg} / \mathrm{Y}$ Algal oil denisty $=920 \mathrm{~kg} / \mathrm{m} 3$

Total oil produced $=[(481,140,000 \mathrm{~kg}) /(920 \mathrm{~kg} / \mathrm{m3})] \times(1000 \mathrm{~L} / \mathrm{m3})=522,978,260.90 \mathrm{~L} / \mathrm{Y}$

COP of AJF $=(\$ 0.90 / \mathrm{L})+(\$ 0.34 / \mathrm{L})=\$ 1.24 / \mathrm{L}$

Total COP $=(522,978,260.90 \mathrm{~L} / \mathrm{Y}) \times(\$ 1.24 / \mathrm{L})=\$ 648,493,043.50$

Total Revenue from Sale of AJF $=522,978,260.90 \mathrm{~L} / \mathrm{Y}) \times(\$ 1.00 / \mathrm{L})=\$ 522,978,260.90$

Hypothetical Jetfuel price $=\$ 1 / \mathrm{L}(\$ 158.76 / \mathrm{bbl})$

Emissions price $=\$ 30 / \mathrm{tCO} 2 \mathrm{e}$

Energy Density of Bio-SPK $=43.5 \mathrm{MJ} / \mathrm{kg}$

Total production per year by energy $(\mathrm{MJ})=(481,140,000 \mathrm{Kg} / \mathrm{Y}) \times(43.5 \mathrm{MJ} / \mathrm{kg})=2,092,9590,000 \mathrm{MJ}$

Life-Cylce Emission intensity of AJF $=25 \mathrm{gCO} e / \mathrm{MJ}$

Total Life-Cycle Emission per year from AJF $=(2,092,9590,000 \mathrm{MJ}) \times(25 \mathrm{gCO} e / \mathrm{MJ})=523,239,750,000 \mathrm{gCO} 2 \mathrm{e}$

Life-Cycle Emissions intensity of PJF $=87.5 \mathrm{gCO} e / \mathrm{MJ}$

Total Life-Cycle Emission per year from PJF $=(2,092,9590,000 \mathrm{MJ}) \times(87.5 \mathrm{gCO} e / \mathrm{MJ})=1,831,339,125,000 \mathrm{gCO} 2 \mathrm{e}$

Total Life-Cycle Emission benefit per year $=(1,831,339,125,000)-(523,239,750,000)=1,308,099,375,000$ or 1,308,099.38 tCO2

Total Monetized Life-Cycle Emission Benefit per year $=(1,308,099.38$ tCO2 $) \times(\$ 30 / \mathrm{tCO})=\$ 39,242,981.25$

$\mathrm{WACC}=7 \%$

Total UCC $=\$ 947.3 \mathrm{M}$ 


\section{Calculations for Changing Algal Oil Content, Productivity \& JFP - @ 60\%,60 g/m2/day \&}

$\$ 1.50 / 1$

\begin{tabular}{lccccccccccc}
\multicolumn{1}{r}{ Source of Cash Flow (\$M) } & $\mathbf{0}$ & $\mathbf{1}$ & $\mathbf{2}$ & $\mathbf{3}$ & $\mathbf{4}$ & $\mathbf{5}$ & $\mathbf{6}$ & $\mathbf{7}$ & $\mathbf{8}$ & $\mathbf{9}$ & $\mathbf{1 0}$ \\
Total Revenue from sale of AJF & 0 & 784.47 & 784.47 & 784.47 & 784.47 & 784.5 & 784 & 784.5 & 784.5 & 784.5 & 784.47 \\
Total Revenue from emissions benefit & 0 & 39.24 & 39.24 & 39.24 & 39.24 & 39.24 & 39.2 & 39.24 & 39.24 & 39.24 & 39.24 \\
Total COP of AJF & 0 & 648.49 & 648.49 & 648.49 & 648.49 & 648.5 & 648 & 648.5 & 648.5 & 648.5 & 648.49 \\
EBITDA & 0 & 175.22 & 175.22 & 175.22 & 175.22 & 175.2 & 175 & 175.2 & 175.2 & 175.2 & 175.22 \\
Discount Factor & 1.00 & 1.07 & 1.14 & 1.23 & 1.31 & 1.40 & 1.50 & 1.61 & 1.72 & 1.84 & 1.97 \\
Discounted EBITDA & 0.00 & 163.76 & 153.04 & 143.03 & 133.67 & 124.93 & 116.76 & 109.12 & 101.98 & 95.31 & 89.07 \\
Terminal Value & & & & & & & & & & & \\
Upfront Capital Cost & -947.30 & & & & & & & & & &
\end{tabular}

\section{ASSUMPTIONS:}

Algae oil content $=60 \%$

System productivity $=60 \mathrm{~g} / \mathrm{m} 2 /$ day

Total pond size $=4050 \mathrm{ha}$

Total algal oil produced $=[(60 \mathrm{~g} / \mathrm{m} 2 /$ day $) \times(10,000 \mathrm{~m} 2 / \mathrm{ha}) \times(4050 \mathrm{ha}) \times(0.60 \mathrm{gAlgal}-\mathrm{oi} / \mathrm{gAlgae}) \times(330 \mathrm{days} / \mathrm{year})] /[1000 \mathrm{~g} / \mathrm{kg}]=481,140,000 \mathrm{Kg} / \mathrm{Y}$ Algal oil denisty $=920 \mathrm{~kg} / \mathrm{m3}$

Total oil produced $=[(481,140,000 \mathrm{~kg}) /(920 \mathrm{~kg} / \mathrm{m3})] \times(1000 \mathrm{~L} / \mathrm{m} 3)=522,978,260.90 \mathrm{~L} / \mathrm{Y}$

COP of $A J F=(\$ 0.90 / L)+(\$ 0.34 / L)=\$ 1.24 / L$

Total COP $=(522,978,260.90 \mathrm{~L} / \mathrm{Y}) \times(\$ 1.24 / \mathrm{L})=\$ 648,493,043.50$

Total Revenue from Sale of AJF $=522,978,260.90 \mathrm{~L} / \mathrm{Y}) \times(\$ 1.50 / \mathrm{L})=\$ 784,467.391 .40$

Hypothetical Jetfuel price $=\$ 1.50 / \mathrm{L}(\$ 238.14 / \mathrm{bb})$

Emissions price $=\$ 30 / \mathrm{tCO} 2 \mathrm{e}$

Energy Density of Bio-SPK $=43.5 \mathrm{MJ} / \mathrm{kg}$

Total production per year by energy $(\mathrm{MJ})=(481,140,000 \mathrm{Kg} / \mathrm{Y}) \times(43.5 \mathrm{MJ} / \mathrm{kg})=2,092,9590,000 \mathrm{MJ}$

Life-Cylce Emission intensity of AJF $=25$ gCO2e/MJ

Total Life-Cycle Emission per year from AJF $=(2,092,9590,000 \mathrm{MJ}) \times(25 \mathrm{gCO} e / \mathrm{MJ})=523,239,750,000 \mathrm{gCO} e$

Life-Cycle Emissions intensity of PJF $=87.5 \mathrm{gCO} e / \mathrm{MJ}$

Total Life-Cycle Emission per year from PJF $=(2,092,9590,000 \mathrm{MJ}) \times(87.5 \mathrm{gCO} e / \mathrm{MJ})=1,831,339,125,000 \mathrm{gCO} \mathrm{e}$

Total Life-Cycle Emission benefit per year $=(1,831,339,125,000)-(523,239,750,000)=1,308,099,375,000$ or $1,308,099.38$ tCO2

Total Monetized Life-Cycle Emission Benefit per year $=(1,308,099.38 \mathrm{tCO}) \times(\$ 30 / \mathrm{tCO} 2)=\$ 39,242,981.25$

WACC $=7 \%$

Total UCC $=\$ 947.3 \mathrm{M}$ 


\section{Calculations for Changing Algal Oil Content, Productivity \& JFP - @ 60\%, 60 g/m2/day \& $\$ 3 / 1$}

\begin{tabular}{|c|c|c|c|c|c|c|c|c|c|c|c|}
\hline \multirow[b]{2}{*}{ Source of Cash Flow (\$M) } & \multicolumn{11}{|c|}{ Year (N) } \\
\hline & 0 & 1 & 2 & 3 & 4 & 5 & 6 & 7 & 8 & 9 & 10 \\
\hline Total Revenue from sale of AJF & 0 & $1,568.93$ & $1,568.93$ & $1,568.93$ & $1,568.93$ & $1,568.93$ & $1,568.93$ & $1,568.93$ & \#\#\#\#\#\#\# & \#\#\#\#\#\#\# & $1,568.93$ \\
\hline Total Revenue from emissions benefit & 0 & 39.24 & 39.24 & 39.24 & 39.24 & 39.24 & 39.24 & 39.24 & 39.24 & 39.24 & 39.24 \\
\hline Total COP of AJF & 0 & 648.49 & 648.49 & 648.49 & 648.49 & 648.5 & 648.49 & 648.49 & 648.5 & 648.5 & 648.49 \\
\hline EBITDA & 0 & 959.68 & 959.68 & 959.68 & 959.68 & 959.7 & 959.68 & 959.68 & 959.7 & 959.7 & 959.68 \\
\hline Discount Factor & 1.00 & 1.07 & 1.14 & 1.23 & 1.31 & 1.40 & 1.50 & 1.61 & 1.72 & 1.84 & 1.97 \\
\hline Discounted EBITDA & 0.00 & 896.90 & 838.22 & 783.38 & 732.14 & 684.24 & 639.48 & 597.64 & 558.54 & 522.00 & 487.85 \\
\hline Terminal Value & & & & & & & & & & & 13709.71 \\
\hline Upfront Capital Cost & -947.30 & & & & & & & & & & \\
\hline NPV & 19502.81 & & & & & & & & & & \\
\hline
\end{tabular}

\section{ASSUMPTIONS:}

Algae oil content $=60 \%$

System productivity $=60 \mathrm{~g} / \mathrm{m} 2 /$ day

Total pond size $=4050$ ha

Total algal oil produced $=[(60 \mathrm{~g} / \mathrm{m} 2 /$ day $) \times(10,000 \mathrm{~m} 2 / \mathrm{ha}) \times(4050 \mathrm{ha}) \times(0.60 \mathrm{gAlgal}-\mathrm{oi} / \mathrm{gAlgae}) \times(330 \mathrm{days} /$ year $)] /[1000 \mathrm{~g} / \mathrm{kg}]=481,140,000 \mathrm{Kg} / \mathrm{Y}$ Algal oil denisty $=920 \mathrm{~kg} / \mathrm{m} 3$

Total oil produced $=[(481,140,000 \mathrm{~kg}) /(920 \mathrm{~kg} / \mathrm{m3})] \times(1000 \mathrm{~L} / \mathrm{m3})=522,978,260.90 \mathrm{~L} / \mathrm{Y}$

COP of AJF $=(\$ 0.90 / \mathrm{L})+(\$ 0.34 / \mathrm{L})=\$ 1.24 / \mathrm{L}$

Total COP $=(522,978,260.90 \mathrm{~L} / \mathrm{Y}) \times(\$ 1.24 / \mathrm{L})=\$ 648,493,043.50$

Total Revenue from Sale of AJF $=522,978,260.90 \mathrm{~L} / \mathrm{Y}) \times(\$ 3 / \mathrm{L})=\$ 1,568,934,783$

Hypothetical Jetfuel price $=\$ 3 / \mathrm{L}(\$ 476.28 / \mathrm{bb})$

Emissions price $=\$ 30 / \mathrm{tCO} 2 \mathrm{e}$

Energy Density of Bio-SPK $=43.5 \mathrm{MJ} / \mathrm{kg}$

Total production per year by energy $(\mathrm{MJ})=(481,140,000 \mathrm{Kg} / \mathrm{Y}) \times(43.5 \mathrm{MJ} / \mathrm{kg})=2,092,9590,000 \mathrm{MJ}$

Life-Cylce Emission intensity of AJF $=25 \mathrm{gCO} e / \mathrm{MJ}$

Total Life-Cycle Emission per year from AJF $=(2,092,9590,000 \mathrm{MJ}) \times(25 \mathrm{gCO} e / \mathrm{MJ})=523,239,750,000 \mathrm{gCO} e$

Life-Cycle Emissions intensity of PJF $=87.5 \mathrm{gCO} e / \mathrm{MJ}$

Total Life-Cycle Emission per year from PJF $=(2,092,9590,000 \mathrm{MJ}) \times(87.5 \mathrm{gCO} 2 \mathrm{e} / \mathrm{MJ})=1,831,339,125,000 \mathrm{gCO} 2 \mathrm{e}$

Total Life-Cycle Emission benefit per year $=(1,831,339,125,000)-(523,239,750,000)=1,308,099,375,000$ or 1,308,099.38 tCO2

Total Monetized Life-Cycle Emission Benefit per year $=(1,308,099.38 \mathrm{tCO}) \times(\$ 30 / \mathrm{tCO})=\$ 39,242,981.25$

WACC $=7 \%$

Total UCC $=\$ 947.3 \mathrm{M}$ 


\section{APPENDIX G}

Calculations for Algal Oil Content, Productivity \& Carbon Tax - @ 50\%, 40 g/m²/day \& $\$ 60 / \mathrm{tCO}_{2} \underline{\mathrm{e}}$

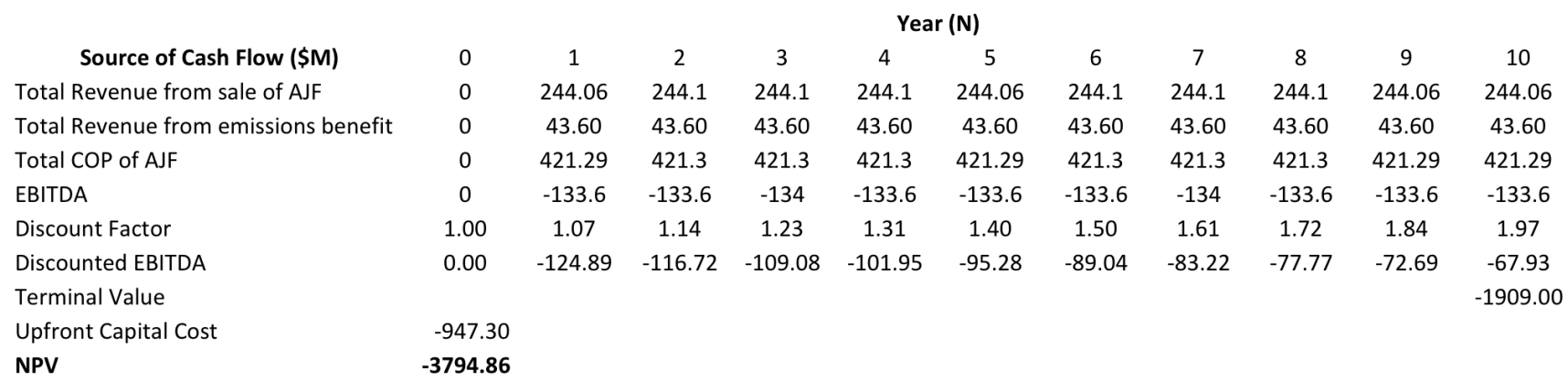

\section{ASSUMPTIONS:}

Algae oil content $=50 \%$

System productivity $=40 \mathrm{~g} / \mathrm{m} 2 /$ day

Total pond size $=4050$ ha

Total algal oil produced $=[(40 \mathrm{~g} / \mathrm{m} 2 /$ day $) \times(10,000 \mathrm{~m} 2 / \mathrm{ha}) \times(4050 \mathrm{ha}) \times(0.50 \mathrm{gAlgal}-\mathrm{oil} / \mathrm{gAlgae}) \times(330$ days $/$ year $)] /[1000 \mathrm{~g} / \mathrm{kg}]=267,300,000 \mathrm{Kg} / \mathrm{Y}$ Algal oil denisty $=920 \mathrm{~kg} / \mathrm{m} 3$

Total oil produced $=[(267,300,000 \mathrm{~kg}) /(920 \mathrm{~kg} / \mathrm{m3})] \times(1000 \mathrm{~L} / \mathrm{m3})=290,543,478.30 \mathrm{~L} / \mathrm{Y}$

COP of AJF $=(\$ 1.11 / \mathrm{L})+(\$ 0.34 / \mathrm{L})=\$ 1.45 / \mathrm{L}$

Total COP $=(290,543.478 .30 \mathrm{~L} / \mathrm{Y}) \times(\$ 1.45 / \mathrm{L})=\$ 421,288,043.50$

Total Revenue from Sale of AJF $=290,543.478 .30 \mathrm{~L} / \mathrm{Y}) \times(\$ 0.84 / \mathrm{L})=\$ 244,056,521,70$

Average price of Jetfuel for $2013=\$ 132.80 / \mathrm{bbl}$ or $\$ 0.84 / \mathrm{L}$

Emissions price $=\$ 60 / \mathrm{tCO} 2 \mathrm{e}$

Energy Density of Bio-SPK $=43.5 \mathrm{MJ} / \mathrm{kg}$

Total production per year by energy $(\mathrm{MJ})=(267,300,000 \mathrm{Kg} / \mathrm{Y}) \times(43.5 \mathrm{MJ} / \mathrm{kg})=11,627,550,000 \mathrm{MJ}$

Life-Cylce Emission intensity of AJF $=25 \mathrm{gCO} 2 \mathrm{e} / \mathrm{MJ}$

Total Life-Cycle Emission per year from AJF $=(11,627,550,000 \mathrm{MJ}) \times(25 \mathrm{gCO} e / \mathrm{MJ})=290,688,750,000 \mathrm{gCO} 2 \mathrm{e}$

Life-Cycle Emissions intensity of PJF $=87.5 \mathrm{gCO} 2 \mathrm{e} / \mathrm{M}$

Total Life-Cycle Emission per year from PJF $=(11,627,550,000 \mathrm{MJ}) \times(87.5 \mathrm{gCO} e / \mathrm{MJ})=1,017,410,625,000 \mathrm{gCO} \mathrm{e}$

Total Life-Cycle Emission benefit per year $=(1,017,410,625,000)-(290,688,750,000)=726,721,875,000$ or $726,721.88$ tCO2

Total Monetized Life-Cycle Emission Benefit per year $=(726,721.88 \mathrm{tCO}) \times(\$ 60 / \mathrm{tCO})=\$ 43,603,312.80$

WACC $=7 \%$

Total UCC $=\$ 947.3 \mathrm{M}$ 


\section{Calculations for Algal Oil Content, Productivity \& Carbon Tax - @ 50\%, $40 \mathrm{~g} / \mathrm{m}^{2} /$ day \& $\$ 120 / \mathrm{tCO}_{2} \mathrm{e}$}

\begin{tabular}{|c|c|c|c|c|c|c|c|c|c|c|c|}
\hline \multirow[b]{2}{*}{ Source of Cash Flow (\$M) } & \multicolumn{11}{|c|}{ Year (N) } \\
\hline & 0 & 1 & 2 & 3 & 4 & 5 & 6 & 7 & 8 & 9 & 10 \\
\hline Total Revenue from emissions benefit & 0 & 87.21 & 87.21 & 87.21 & 87.21 & 87.21 & 87.21 & 87.21 & 87.21 & 87.21 & 87.21 \\
\hline EBITDA & 0 & -90.02 & -90.02 & -90 & -90.02 & -90.02 & -90.02 & -90 & -90.02 & -90.02 & -90.02 \\
\hline Discount Factor & 1.00 & 1.07 & 1.14 & 1.23 & 1.31 & 1.40 & 1.50 & 1.61 & 1.72 & 1.84 & 1.97 \\
\hline Discounted EBITDA & 0.00 & -84.13 & -78.63 & -73.48 & -68.68 & -64.18 & -59.98 & -56.06 & -52.39 & -48.96 & -45.76 \\
\hline Upfront Capital Cost & -947.30 & & & & & & & & & & \\
\hline NPV & -2865.56 & & & & & & & & & & \\
\hline
\end{tabular}

\section{ASSUMPTIONS:}

Algae oil content $=50 \%$

System productivity $=40 \mathrm{~g} / \mathrm{m} 2 /$ day

Total pond size $=4050$ ha

Total algal oil produced $=[(40 \mathrm{~g} / \mathrm{m} 2 /$ day $) \times(10,000 \mathrm{~m} 2 / \mathrm{ha}) \times(4050 \mathrm{ha}) \times(0.50 \mathrm{gAlgal}-$ oil $/ \mathrm{gAlgae}) \times(330$ days $/$ year $)] /[1000 \mathrm{~g} / \mathrm{kg}]=267,300,000 \mathrm{Kg} / \mathrm{Y}$ Algal oil denisty $=920 \mathrm{~kg} / \mathrm{m3}$

Total oil produced $=[(267,300,000 \mathrm{~kg}) /(920 \mathrm{~kg} / \mathrm{m3})] \times(1000 \mathrm{~L} / \mathrm{m3})=290,543,478.30 \mathrm{~L} / \mathrm{Y}$

COP of AJF $=(\$ 1.11 / \mathrm{L})+(\$ 0.34 / \mathrm{L})=\$ 1.45 / \mathrm{L}$

Total COP $=(290,543.478 .30 \mathrm{~L} / \mathrm{Y}) \times(\$ 1.45 / \mathrm{L})=\$ 421,288,043.50$

Total Revenue from Sale of AJF $=290,543.478 .30 \mathrm{~L} / \mathrm{Y}) \times(\$ 0.84 / \mathrm{L})=\$ 244,056,521,70$

Average price of Jetfuel for $2013=\$ 132.80 / \mathrm{bbl}$ or $\$ 0.84 / \mathrm{L}$

Emissions price $=\$ 120 / \mathrm{tCO} 2 \mathrm{e}$

Energy Density of Bio-SPK $=43.5 \mathrm{MJ} / \mathrm{kg}$

Total production per year by energy $(\mathrm{MJ})=(267,300,000 \mathrm{Kg} / \mathrm{Y}) \times(43.5 \mathrm{MJ} / \mathrm{kg})=11,627,550,000 \mathrm{MJ}$

Life-Cylce Emission intensity of AJF $=25 \mathrm{gCO} e / \mathrm{MJ}$

Total Life-Cycle Emission per year from AJF $=(11,627,550,000 \mathrm{MJ}) \times(25 \mathrm{gCO} e / \mathrm{MJ})=290,688,750,000 \mathrm{gCO} e$

Life-Cycle Emissions intensity of PJF $=87.5 \mathrm{gCO} e / \mathrm{MJ}$

Total Life-Cycle Emission per year from PJF $=(11,627,550,000 \mathrm{MJ}) \times(87.5 \mathrm{gCO} 2 \mathrm{e} / \mathrm{MJ})=1,017,410,625,000 \mathrm{gCO} \mathrm{e}$

Total Life-Cycle Emission benefit per year $=(1,017,410,625,000)-(290,688,750,000)=726,721,875,000$ or $726,721.88$ tCO2

Total Monetized Life-Cycle Emission Benefit per year $=(726,721.88 \mathrm{tCO}) \times(\$ 120 / \mathrm{tCO})=\$ 87,206,625.60$

WACC $=7 \%$

Total UCC $=\$ 947.3 \mathrm{M}$ 


\section{Calculations for Algal Oil Content, Productivity \& Carbon Tax - @ 50\%, $40 \mathrm{~g} / \mathrm{m}^{2} /$ day \& $\$ 200 / \mathrm{tCO}_{2} \mathrm{e}$}

\begin{tabular}{|c|c|c|c|c|c|c|c|c|c|c|c|}
\hline \multirow[b]{2}{*}{ Source of Cash Flow (\$M) } & \multicolumn{11}{|c|}{ Year $(\mathrm{N})$} \\
\hline & 0 & 1 & 2 & 3 & 4 & 5 & 6 & 7 & 8 & 9 & 10 \\
\hline Total Revenue from sale of AJF & 0 & 244.06 & 244.1 & 244.1 & 244.1 & 244.06 & 244.1 & 244.1 & 244.1 & 244.06 & 244.06 \\
\hline Total Revenue from emissions benefit & 0 & 145.34 & 145.34 & 145.34 & 145.34 & 145.34 & 145.34 & 145.34 & 145.34 & 145.34 & 145.34 \\
\hline Total COP of AJF & 0 & 421.29 & 421.3 & 421.3 & 421.3 & 421.29 & 421.3 & 421.3 & 421.3 & 421.29 & 421.25 \\
\hline EBITDA & 0 & -31.89 & -31.89 & -31.9 & -31.89 & -31.89 & -31.89 & -31.9 & -31.89 & -31.89 & -31.89 \\
\hline Discount Factor & 1.00 & 1.07 & 1.14 & 1.23 & 1.31 & 1.40 & 1.50 & 1.61 & 1.72 & 1.84 & 1.97 \\
\hline Discounted EBITDA & 0.00 & -29.80 & -27.85 & -26.03 & -24.33 & -22.74 & -21.25 & -19.86 & -18.56 & -17.35 & -16.21 \\
\hline Terminal Value & & & & & & & & & & & -455.57 \\
\hline Upfront Capital Cost & -947.30 & & & & & & & & & & \\
\hline NPV & -1626.85 & & & & & & & & & & \\
\hline
\end{tabular}

\section{ASSUMPTIONS:}

Algae oil content $=50 \%$

System productivity $=40 \mathrm{~g} / \mathrm{m} 2 /$ day

Total pond size $=4050$ ha

Total algal oil produced $=[(40 \mathrm{~g} / \mathrm{m} 2 /$ day $) \times(10,000 \mathrm{~m} 2 / \mathrm{ha}) \times(4050 \mathrm{ha}) \times(0.50 \mathrm{gAlgal}-$ oil $/$ gAlgae $) \times(330$ days $/$ year $)] /[1000 \mathrm{~g} / \mathrm{kg}]=267,300,000 \mathrm{Kg} / \mathrm{Y}$

Algal oil denisty $=920 \mathrm{~kg} / \mathrm{m} 3$

Total oil produced $=[(267,300,000 \mathrm{~kg}) /(920 \mathrm{~kg} / \mathrm{m3})] \times(1000 \mathrm{~L} / \mathrm{m3})=290,543,478.30 \mathrm{~L} / \mathrm{Y}$

COP of AJF $=(\$ 1.11 / \mathrm{L})+(\$ 0.34 / \mathrm{L})=\$ 1.45 / \mathrm{L}$

Total COP $=(290,543.478 .30 \mathrm{~L} / \mathrm{Y}) \times(\$ 1.45 / \mathrm{L})=\$ 421,288,043.50$

Total Revenue from Sale of AJF $=290,543.478 .30 \mathrm{~L} / \mathrm{Y}) \times(\$ 0.84 / \mathrm{L})=\$ 244,056,521,70$

Average price of Jetfuel for $2013=\$ 132.80 / \mathrm{bbl}$ or $\$ 0.84 / \mathrm{L}$

Emissions price $=\$ 200 / \mathrm{tCO} \mathrm{e}$

Energy Density of Bio-SPK $=43.5 \mathrm{MJ} / \mathrm{kg}$

Total production per year by energy $(\mathrm{MJ})=(267,300,000 \mathrm{Kg} / \mathrm{Y}) \times(43.5 \mathrm{MJ} / \mathrm{kg})=11,627,550,000 \mathrm{MJ}$

Life-Cylce Emission intensity of AJF $=25 \mathrm{gCO} 2 \mathrm{e} / \mathrm{MJ}$

Total Life-Cycle Emission per year from AJF $=(11,627,550,000 \mathrm{MJ}) \times(25 \mathrm{gCO} e / \mathrm{MJ})=290,688,750,000 \mathrm{gCO} e$

Life-Cycle Emissions intensity of PJF $=87.5 \mathrm{gCO} 2 \mathrm{e} / \mathrm{MJ}$

Total Life-Cycle Emission per year from PJF $=(11,627,550,000 \mathrm{MJ}) \times(87.5 \mathrm{gCO} e / \mathrm{MJ})=1,017,410,625,000 \mathrm{gCO} \mathrm{e}$

Total Life-Cycle Emission benefit per year $=(1,017,410,625,000)-(290,688,750,000)=726,721,875,000$ or $726,721.88$ tCO2

Total Monetized Life-Cycle Emission Benefit per year $=(726,721.88 \mathrm{tCO}) \times(\$ 200 / \mathrm{tCO})=\$ 145,344,376$

WACC $=7 \%$

Total UCC $=\$ 947.3 \mathrm{M}$ 


\section{Calculations for Algal Oil Content, Productivity \& Carbon Tax - @ 50\%, $40 \mathrm{~g} / \mathrm{m}^{2} / \mathrm{day} \&$ $\$ 400 / \mathrm{tCO}_{2} \mathrm{e}$}

\begin{tabular}{|c|c|c|c|c|c|c|c|c|c|c|c|}
\hline \multirow[b]{2}{*}{ Source of Cash Flow (\$M) } & \multicolumn{10}{|c|}{ Year (N) } & \multirow[b]{2}{*}{10} \\
\hline & 0 & 1 & 2 & 3 & 4 & 5 & 6 & 7 & 8 & 9 & \\
\hline Total Revenue from sale of AJF & 0 & 244.06 & 244.1 & 244.1 & 244.1 & 244.06 & 244.1 & 244.1 & 244.1 & 244.06 & 244.06 \\
\hline Total Revenue from emissions benefit & 0 & 290.69 & 290.69 & 290.69 & 290.69 & 290.69 & 290.69 & 290.69 & 290.69 & 290.69 & 290.69 \\
\hline Total COP of AJF & 0 & 421.29 & 421.3 & 421.3 & 421.3 & 421.29 & 421.3 & 421.3 & 421.3 & 421.29 & 421.29 \\
\hline EBITDA & 0 & 113.46 & 113.5 & 113.5 & 113.5 & 113.46 & 113.5 & 113.5 & 113.5 & 113.46 & 113.46 \\
\hline Discount Factor & 1.00 & 1.07 & 1.14 & 1.23 & 1.31 & 1.40 & 1.50 & 1.61 & 1.72 & 1.84 & 1.97 \\
\hline Discounted EBITDA & 0.00 & 106.04 & 99.10 & 92.62 & 86.56 & 80.90 & 75.60 & 70.66 & 66.03 & 61.71 & 57.68 \\
\hline Terminal Value & & & & & & & & & & & 1620.86 \\
\hline Upfront Capital Cost & -947.30 & & & & & & & & & & \\
\hline NPV & 1470.45 & & & & & & & & & & \\
\hline
\end{tabular}

ASSUMPTIONS:

Algae oil content $=50 \%$

System productivity $=40 \mathrm{~g} / \mathrm{m} 2 /$ day

Total pond size $=4050$ ha

Total algal oil produced $=[(40 \mathrm{~g} / \mathrm{m} 2 /$ day $) \times(10,000 \mathrm{~m} 2 / \mathrm{ha}) \times(4050 \mathrm{ha}) \times(0.50 \mathrm{gAlgal}-$ oil $/ \mathrm{gAlgae}) \times(330 \mathrm{days} / \mathrm{year})] /[1000 \mathrm{~g} / \mathrm{kg}]=267,300,000 \mathrm{Kg} / \mathrm{Y}$

Algal oil denisty $=920 \mathrm{~kg} / \mathrm{m} 3$

Total oil produced $=[(267,300,000 \mathrm{~kg}) /(920 \mathrm{~kg} / \mathrm{m3})] \times(1000 \mathrm{~L} / \mathrm{m} 3)=290,543,478.30 \mathrm{~L} / \mathrm{Y}$

COP of AJF $=(\$ 1.11 / \mathrm{L})+(\$ 0.34 / \mathrm{L})=\$ 1.45 / \mathrm{L}$

Total COP $=(290,543.478 .30 \mathrm{~L} / \mathrm{Y}) \times(\$ 1.45 / \mathrm{L})=\$ 421,288,043.50$

Total Revenue from Sale of AJF $=290,543.478 .30 \mathrm{~L} / \mathrm{Y}) \times(\$ 0.84 / \mathrm{L})=\$ 244,056,521,70$

Average price of Jetfuel for $2013=\$ 132.80 / \mathrm{bbl}$ or $\$ 0.84 / \mathrm{L}$

Emissions price $=\$ 400 / \mathrm{tCO} 2 \mathrm{e}$

Energy Density of Bio-SPK $=43.5 \mathrm{MJ} / \mathrm{kg}$

Total production per year by energy $(\mathrm{MJ})=(267,300,000 \mathrm{Kg} / \mathrm{Y}) \times(43.5 \mathrm{MJ} / \mathrm{kg})=11,627,550,000 \mathrm{MJ}$

Life-Cylce Emission intensity of AJF $=25 \mathrm{gCO} e / \mathrm{MJ}$

Total Life-Cycle Emission per year from AJF $=(11,627,550,000 \mathrm{MJ}) \times(25 \mathrm{gCO} e / \mathrm{MJ})=290,688,750,000 \mathrm{gCO} e$

Life-Cycle Emissions intensity of PJF $=87.5$ gCO2e/MJ

Total Life-Cycle Emission per year from PJF $=(11,627,550,000 \mathrm{MJ}) \times(87.5 \mathrm{gCO} \mathrm{e} / \mathrm{MJ})=1,017,410,625,000 \mathrm{gCO} \mathrm{e}$

Total Life-Cycle Emission benefit per year $=(1,017,410,625,000)-(290,688,750,000)=726,721,875,000$ or $726,721.88$ tCO2

Total Monetized Life-Cycle Emission Benefit per year $=(726,721.88 \mathrm{tCO}) \times(\$ 400 / \mathrm{tCO})=\$ 290,688,752$

WACC $=7 \%$

Total UCC $=\$ 947.3 \mathrm{M}$ 


\section{Calculations for Algal Oil Content, Productivity \& Carbon Tax - @ 50\%, $40 \mathrm{~g} / \mathrm{m}^{2} / \mathrm{day} \&$ $\$ 800 / \mathrm{tCO}_{2} \mathrm{e}$}

\begin{tabular}{|c|c|c|c|c|c|c|c|c|c|c|}
\hline \multirow[b]{2}{*}{ Source of Cash Flow (\$M) } & \multicolumn{10}{|c|}{ Year (N) } \\
\hline & 0 & 1 & 2 & 3 & 4 & 5 & 6 & 7 & 8 & 9 \\
\hline Total Revenue from sale of AJF & 0 & 244.06 & 244.1 & 244.1 & 244.1 & 244.06 & 244.1 & 244.1 & 244.1 & 244.06 \\
\hline Total Revenue from emissions benefit & 0 & 581.34 & 581.34 & 581.34 & 581.34 & 581.34 & 581.34 & 581.34 & 581.34 & 581.34 \\
\hline Total COP of AJF & 0 & 421.29 & 421.3 & 421.3 & 421.3 & 421.29 & 421.3 & 421.3 & 421.3 & 421.29 \\
\hline EBITDA & 0 & 404.11 & 404.1 & 404.1 & 404.1 & 404.11 & 404.1 & 404.1 & 404.1 & 404.11 \\
\hline Discount Factor & 1.00 & 1.07 & 1.14 & 1.23 & 1.31 & 1.40 & 1.50 & 1.61 & 1.72 & 1.84 \\
\hline Discounted EBITDA & 0.00 & 377.67 & 352.97 & 329.87 & 308.29 & 288.12 & 269.28 & 251.66 & 235.20 & 219.81 \\
\hline \multicolumn{11}{|l|}{ Terminal Value } \\
\hline Upfront Capital Cost & \multicolumn{10}{|l|}{-947.30} \\
\hline NPV & \multicolumn{10}{|l|}{7664.00} \\
\hline \multicolumn{11}{|l|}{ ASSUMPTIONS: } \\
\hline \multicolumn{11}{|l|}{ Algae oil content $=50 \%$} \\
\hline \multicolumn{11}{|l|}{ System productivity $=40 \mathrm{~g} / \mathrm{m} 2 /$ day } \\
\hline \multicolumn{11}{|l|}{ Total pond size $=4050 \mathrm{ha}$} \\
\hline \multicolumn{11}{|c|}{ Total algal oil produced $=[(40 \mathrm{~g} / \mathrm{m} 2 /$ day $) \times(10,000 \mathrm{~m} 2 / \mathrm{ha}) \times(4050 \mathrm{ha}) \times(0.50 \mathrm{gAlgal}$-oil $/ \mathrm{gAlgae}) \times(330 \mathrm{days} /$ year $)] /[1000 \mathrm{~g} / \mathrm{kg}]=267,300,000 \mathrm{Kg} /$} \\
\hline \multicolumn{11}{|l|}{ Algal oil denisty $=920 \mathrm{~kg} / \mathrm{m3}$} \\
\hline \multicolumn{11}{|c|}{ Total oil produced $=[(267,300,000 \mathrm{~kg}) /(920 \mathrm{~kg} / \mathrm{m3})] \times(1000 \mathrm{~L} / \mathrm{m} 3)=290,543,478.30 \mathrm{~L} / \mathrm{Y}$} \\
\hline \multicolumn{11}{|c|}{ COP of $A J F=(\$ 1.11 / L)+(\$ 0.34 / L)=\$ 1.45 / L$} \\
\hline \multicolumn{11}{|c|}{ Total COP $=(290,543.478 .30 \mathrm{~L} / \mathrm{Y}) \times(\$ 1.45 / \mathrm{L})=\$ 421,288,043.50$} \\
\hline \multicolumn{11}{|c|}{ Total Revenue from Sale of AJF $=290,543.478 .30 \mathrm{~L} / \mathrm{Y}) \times(\$ 0.84 / \mathrm{L})=\$ 244,056,521,70$} \\
\hline \multicolumn{11}{|c|}{ Average price of Jetfuel for $2013=\$ 132.80 / \mathrm{bbl}$ or $\$ 0.84 / \mathrm{L}$} \\
\hline \multicolumn{11}{|l|}{ Emissions price $=\$ 800 / \mathrm{tCO} 2 \mathrm{e}$} \\
\hline \multicolumn{11}{|l|}{ Energy Density of Bio-SPK = $43.5 \mathrm{MJ} / \mathrm{kg}$} \\
\hline \multicolumn{11}{|c|}{ Total production per year by energy $(\mathrm{MJ})=(267,300,000 \mathrm{Kg} / \mathrm{Y}) \times(43.5 \mathrm{MJ} / \mathrm{kg})=11,627,550,000 \mathrm{MJ}$} \\
\hline \multicolumn{11}{|c|}{ Life-Cylce Emission intensity of AJF = $25 \mathrm{gCO} e / \mathrm{MJ}$} \\
\hline \multicolumn{11}{|c|}{ Total Life-Cycle Emission per year from AJF $=(11,627,550,000 \mathrm{MJ}) \times(25 \mathrm{gCO} e / \mathrm{MJ})=290,688,750,000 \mathrm{gCO} 2 \mathrm{e}$} \\
\hline \multicolumn{11}{|c|}{ Life-Cycle Emissions intensity of PJF = 87.5 gCO2e/MJ } \\
\hline \multicolumn{11}{|c|}{ Total Life-Cycle Emission per year from PJF $=(11,627,550,000 \mathrm{MJ}) \times(87.5 \mathrm{gCO} 2 \mathrm{e} / \mathrm{MJ})=1,017,410,625,000 \mathrm{gCO} e$} \\
\hline \multicolumn{11}{|c|}{ Total Life-Cycle Emission benefit per year $=(1,017,410,625,000)-(290,688,750,000)=726,721,875,000$ or $726,721.88 \mathrm{tCO}$} \\
\hline \multicolumn{11}{|c|}{ Total Monetized Life-Cycle Emission Benefit per year $=(726,721.88 \mathrm{tCO} 2) \times(\$ 800 / \mathrm{tCO} 2)=\$ 581,377,504$} \\
\hline \multicolumn{11}{|l|}{ WACC $=7 \%$} \\
\hline tal UCC $=\$ 947.3 \mathrm{~N}$ & & & & & & & & & & \\
\hline
\end{tabular}




\section{Calculations for Algal Oil Content, Productivity \& Carbon Tax - @ 60\%, $60 \mathrm{~g} / \mathrm{m}^{2} / \mathrm{day} \&$ $\$ 60 / \mathrm{tCO}_{2} \mathrm{e}$}

\begin{tabular}{lccccccccccc}
\multicolumn{1}{r}{ Source of Cash Flow (\$M) } & 0 & 1 & 2 & 3 & 4 & 5 & 6 & 7 & 8 & 9 & 10 \\
Total Revenue from sale of AJF & 0 & 439.30 & 439.30 & 439.30 & 439.30 & 439.30 & 439.30 & 439.30 & 439.30 & 439.30 & 439.30 \\
Total Revenue from emissions benefit & 0 & 78.49 & 78.49 & 78.49 & 78.49 & 78.49 & 78.49 & 78.49 & 78.49 & 78.49 & 78.49 \\
Total COP of AJF & 0 & 648.49 & 648.5 & 648.5 & 648.5 & 648.49 & 648.5 & 648.5 & 648.5 & 648.49 & 648.49 \\
EBITDA & 0 & -130.70 & -130.70 & -130.70 & -130.70 & -130.70 & -130.70 & -130.70 & -130.70 & -130.70 & -130.70 \\
Discount Factor & 1.00 & 1.07 & 1.14 & 1.23 & 1.31 & 1.40 & 1.50 & 1.61 & 1.72 & 1.84 & 1.97 \\
Discounted EBITDA & 0.00 & -122.15 & -114.16 & -106.69 & -99.71 & -93.19 & -87.09 & -81.39 & -76.07 & -71.09 & -66.44 \\
Terminal Value & & & & & & & & & & \\
Upfront Capital Cost & -947.30 & & & & & & & & &
\end{tabular}

\section{ASSUMPTIONS:}

Algae oil content $=60 \%$

System productivity $=60 \mathrm{~g} / \mathrm{m} 2 /$ day

Total pond size $=4050$ ha

Total algal oil produced $=[(60 \mathrm{~g} / \mathrm{m} 2 /$ day $) \times(10,000 \mathrm{~m} 2 / \mathrm{ha}) \times(4050 \mathrm{ha}) \times(0.60 \mathrm{gAlgal}-\mathrm{oi} / \mathrm{gAlgae}) \times(330$ days $/$ vear $)] /[1000 \mathrm{~g} / \mathrm{kg}]=481,140,000 \mathrm{Kg} / \mathrm{Y}$ Algal oil denisty $=920 \mathrm{~kg} / \mathrm{m} 3$

Total oil produced $=[(481,140,000 \mathrm{~kg}) /(920 \mathrm{~kg} / \mathrm{m3})] \times(1000 \mathrm{~L} / \mathrm{m} 3)=522,978,260.90 \mathrm{~L} / \mathrm{Y}$

COP of AJF $=(\$ 0.90 / \mathrm{L})+(\$ 0.34 / \mathrm{L})=\$ 1.24 / \mathrm{L}$

Total COP $=(522,978,260.90 \mathrm{~L} / \mathrm{Y}) \times(\$ 1.24 / \mathrm{L})=\$ 648,493,043.50$

Total Revenue from Sale of AJF $=489,460,834.20 \mathrm{~L} / \mathrm{Y}) \times(\$ 0.84 / \mathrm{L})=\$ 439,301,739.10$

Average price of Jetfuel for $2013=\$ 132.80 / \mathrm{bbl}$ or $\$ 0.84 / \mathrm{L}$

Emissions price $=\$ 60 / \mathrm{tCO} 2 \mathrm{e}$

Energy Density of Bio-SPK $=43.5 \mathrm{MJ} / \mathrm{kg}$

Total production per year by energy $(\mathrm{MJ})=(481,140,000 \mathrm{Kg} / \mathrm{Y}) \times(43.5 \mathrm{MJ} / \mathrm{kg})=2,092,9590,000 \mathrm{MJ}$

Life-Cylce Emission intensity of AJF $=25$ gCO2e/MJ

Total Life-Cycle Emission per year from AJF $=(2,092,9590,000 \mathrm{MJ}) \times(25 \mathrm{gCO} e / \mathrm{MJ})=523,239,750,000 \mathrm{gCO} \mathrm{e}$

Life-Cycle Emissions intensity of PJF $=87.5 \mathrm{gCO} 2 \mathrm{e} / \mathrm{MJ}$

Total Life-Cycle Emission per year from PJF $=(2,092,9590,000 \mathrm{MJ}) \times(87.5 \mathrm{gCO} e / \mathrm{MJ})=1,831,339,125,000 \mathrm{gCO} \mathrm{e}$

Total Life-Cycle Emission benefit per year $=(1,831,339,125,000)-(523,239,750,000)=1,308,099,375,000$ or 1,308,099.38 tCO2

Total Monetized Life-Cycle Emission Benefit per year $=(1,308,099.38 \mathrm{tCO}) \times(\$ 60 / \mathrm{tCO})=\$ 78,485,962.80$

WACC $=7 \%$

Total UCC $=\$ 947.3 \mathrm{M}$ 


\section{Calculations for Algal Oil Content, Productivity \& Carbon Tax - @ 60\%, $60 \mathrm{~g} / \mathrm{m}^{2} /$ day \& $\$ 120 / \mathrm{tCO}_{2} \mathrm{e}$}

\begin{tabular}{|c|c|c|c|c|c|c|c|c|c|c|c|}
\hline \multirow[b]{2}{*}{ Source of Cash Flow (\$M) } & \multicolumn{10}{|c|}{ Year (N) } & \multirow[b]{2}{*}{10} \\
\hline & 0 & 1 & 2 & 3 & 4 & 5 & 6 & 7 & 8 & 9 & \\
\hline Total Revenue from sale of AJF & 0 & 439.30 & 439.30 & 439.30 & 439.30 & 439.30 & 439.30 & 439.30 & 439.30 & 439.30 & 439.30 \\
\hline Total Revenue from emissions benefit & 0 & 156.97 & 157 & 157 & 157 & 156.97 & 157 & 157 & 157 & 156.97 & 156.97 \\
\hline Total COP of AJF & 0 & 648.49 & 648.5 & 648.5 & 648.5 & 648.49 & 648.5 & 648.5 & 648.5 & 648.49 & 648.49 \\
\hline EBITDA & 0 & -52.22 & -52.22 & -52.22 & -52.22 & -52.22 & -52.22 & -52.22 & -52.22 & -52.22 & -52.22 \\
\hline Discount Factor & 1.00 & 1.07 & 1.14 & 1.23 & 1.31 & 1.40 & 1.50 & 1.61 & 1.72 & 1.84 & 1.97 \\
\hline Discounted EBITDA & 0.00 & -48.80 & -45.61 & -42.63 & -39.84 & -37.23 & -34.80 & -32.52 & -30.39 & -28.40 & -26.55 \\
\hline Terminal Value & & & & & & & & & & & -746.00 \\
\hline Upfront Capital Cost & -947.30 & & & & & & & & & & \\
\hline NPV & -2060.07 & & & & & & & & & & \\
\hline
\end{tabular}

\section{ASSUMPTIONS:}

Algae oil content $=60 \%$

System productivity $=60 \mathrm{~g} / \mathrm{m} 2 /$ day

Total pond size $=4050$ ha

Total algal oil produced $=[(60 \mathrm{~g} / \mathrm{m} 2 /$ day $) \times(10,000 \mathrm{~m} 2 / \mathrm{ha}) \times(4050 \mathrm{ha}) \times(0.60 \mathrm{gAlgal}-\mathrm{oi} / \mathrm{gAlgae}) \times(330$ days $/$ year $)] /[1000 \mathrm{~g} / \mathrm{kg}]=481,140,000 \mathrm{Kg} / \mathrm{Y}$ Algal oil denisty $=920 \mathrm{~kg} / \mathrm{m3}$

Total oil produced $=[(481,140,000 \mathrm{~kg}) /(920 \mathrm{~kg} / \mathrm{m3})] \times(1000 \mathrm{~L} / \mathrm{m} 3)=522,978,260.90 \mathrm{~L} / \mathrm{Y}$

COP of AJF $=(\$ 0.90 / \mathrm{L})+(\$ 0.34 / \mathrm{L})=\$ 1.24 / \mathrm{L}$

Total COP $=(522,978,260.90 \mathrm{~L} / \mathrm{Y}) \times(\$ 1.24 / \mathrm{L})=\$ 648,493,043.50$

Total Revenue from Sale of AJF $=489,460,834.20 \mathrm{~L} / \mathrm{Y}) \times(\$ 0.84 / \mathrm{L})=\$ 439,301,739.10$

Average price of Jetfuel for $2013=\$ 132.80 / \mathrm{bbl}$ or $\$ 0.84 / \mathrm{L}$

Emissions price $=\$ 120 / \mathrm{tCO} 2 \mathrm{e}$

Energy Density of Bio-SPK $=43.5 \mathrm{MJ} / \mathrm{kg}$

Total production per year by energy $(\mathrm{MJ})=(481,140,000 \mathrm{Kg} / \mathrm{Y}) \times(43.5 \mathrm{MJ} / \mathrm{kg})=2,092,9590,000 \mathrm{MJ}$

Life-Cylce Emission intensity of AJF $=25$ gCO2e/MJ

Total Life-Cycle Emission per year from AJF $=(2,092,9590,000 \mathrm{MJ}) \times(25 \mathrm{gCO} e / \mathrm{MJ})=523,239,750,000 \mathrm{gCO} e$

Life-Cycle Emissions intensity of PJF $=87.5 \mathrm{gCO} e /{ }^{*}$ Boeing 2009

Total Life-Cycle Emission per year from PJF $=(2,092,9590,000 \mathrm{MJ}) \times(87.5 \mathrm{gCO} e / \mathrm{MJ})=1,831,339,125,000 \mathrm{gCO} e$

Total Life-Cycle Emission benefit per year $=(1,831,339,125,000)-(523,239,750,000)=1,308,099,375,000$ or 1,308,099.38 tCO2

Total Monetized Life-Cycle Emission Benefit per year $=(1,308,099.38 \mathrm{tCO} 2) \times(\$ 120 / \mathrm{tCO})=\$ 156,971,925.60$

WACC $=7 \%$

Total UCC $=\$ 947.3 \mathrm{M}$ 


\section{Calculations for Algal Oil Content, Productivity \& Carbon Tax - @ 60\%, $60 \mathrm{~g} / \mathrm{m}^{2} / \mathrm{day} \&$ $\$ 200 / \mathrm{tCO}_{2} \mathrm{e}$}

\begin{tabular}{lccccccccccc}
\multicolumn{1}{r}{ Source of Cash Flow (\$M) } & 0 & 1 & 2 & 3 & 4 & 5 & 6 & 7 & 8 & 9 & 10 \\
Total Revenue from sale of AJF & 0 & 439.30 & 439.30 & 439.30 & 439.30 & 439.30 & 439.30 & 439.30 & 439.30 & 439.30 & 439.30 \\
Total Revenue from emissions benefit & 0 & 261.62 & 261.6 & 261.6 & 261.6 & 261.62 & 261.6 & 261.6 & 261.6 & 261.62 & 261.62 \\
Total COP of AJF & 0 & 648.49 & 648.5 & 648.5 & 648.5 & 648.49 & 648.5 & 648.5 & 648.5 & 648.49 & 648.49 \\
EBITDA & 0 & 52.43 & 52.43 & 52.43 & 52.43 & 52.43 & 52.43 & 52.43 & 52.43 & 52.43 & 52.43 \\
Discount Factor & 1.00 & 1.07 & 1.14 & 1.23 & 1.31 & 1.40 & 1.50 & 1.61 & 1.72 & 1.84 & 1.97 \\
Discounted EBITDA & 0.00 & 49.00 & 45.79 & 42.80 & 40.00 & 37.38 & 34.94 & 32.65 & 30.51 & 28.52 & 26.65 \\
Terminal Value & & & & & & & & & & & \\
Upfront Capital Cost & -947.30 & & & & & & & & & &
\end{tabular}

\section{ASSUMPTIONS:}

Algae oil content $=60 \%$

System productivity $=60 \mathrm{~g} / \mathrm{m} 2 /$ day

Total pond size $=4050$ ha

Total algal oil produced $=[(60 \mathrm{~g} / \mathrm{m} 2 /$ day $) \times(10,000 \mathrm{~m} 2 / \mathrm{ha}) \times(4050 \mathrm{ha}) \times(0.60 \mathrm{gAlgal}-$ oi $/ \mathrm{gAlgae}) \times(330$ days $/$ year $)] /[1000 \mathrm{~g} / \mathrm{kg}]=481,140,000 \mathrm{Kg} / \mathrm{Y}$ Algal oil denisty $=920 \mathrm{~kg} / \mathrm{m3}$

Total oil produced $=[(481,140,000 \mathrm{~kg}) /(920 \mathrm{~kg} / \mathrm{m3})] \times(1000 \mathrm{~L} / \mathrm{m3})=522,978,260.90 \mathrm{~L} / \mathrm{Y}$

COP of AJF $=(\$ 0.90 / \mathrm{L})+(\$ 0.34 / \mathrm{L})=\$ 1.24 / \mathrm{L}$

Total COP $=(522,978,260.90 \mathrm{~L} / \mathrm{Y}) \times(\$ 1.24 / \mathrm{L})=\$ 648,493,043.50$

Total Revenue from Sale of AJF $=489,460,834.20 \mathrm{~L} / \mathrm{Y}) \times(\$ 0.84 / \mathrm{L})=\$ 439,301,739.10$

Average price of Jetfuel for $2013=\$ 132.80 / \mathrm{bbl}$ or $\$ 0.84 / \mathrm{L}$

Emissions price $=\$ 200 / \mathrm{tCO} 2 \mathrm{e}$

Energy Density of Bio-SPK $=43.5 \mathrm{MJ} / \mathrm{kg}$

Total production per year by energy $(\mathrm{MJ})=(481,140,000 \mathrm{Kg} / \mathrm{Y}) \times(43.5 \mathrm{MJ} / \mathrm{kg})=2,092,9590,000 \mathrm{MJ}$

Life-Cylce Emission intensity of AJF $=25 \mathrm{gCO} e / \mathrm{MJ}$

Total Life-Cycle Emission per year from AJF $=(2,092,9590,000 \mathrm{MJ}) \times(25 \mathrm{gCO} e / \mathrm{MJ})=523,239,750,000 \mathrm{gCO} e$

Life-Cycle Emissions intensity of PJF $=87.5 \mathrm{gCO} e / \mathrm{MJ}$

Total Life-Cycle Emission per year from PJF $=(2,092,9590,000 \mathrm{MJ}) \times(87.5 \mathrm{gCO} \mathrm{e} / \mathrm{MJ})=1,831,339,125,000 \mathrm{gCO} \mathrm{e}$

Total Life-Cycle Emission benefit per year $=(1,831,339,125,000)-(523,239,750,000)=1,308,099,375,000$ or 1,308,099.38 tCO2

Total Monetized Life-Cycle Emission Benefit per year $=(1,308,099.38 \mathrm{tCO} 2) \times(\$ 200 / \mathrm{tCO} 2)=\$ 261,619,876$

WACC $=7 \%$

Total UCC $=\$ 947.3 \mathrm{M}$ 


\section{Calculations for Algal Oil Content, Productivity \& Carbon Tax - @ 60\%, $60 \mathrm{~g} / \mathrm{m}^{2} / \mathrm{day} \&$ $\$ 400 / \mathrm{tCO}_{2} \mathrm{e}$}

\begin{tabular}{|c|c|c|c|c|c|c|c|c|c|c|c|}
\hline \multirow[b]{2}{*}{ Source of Cash Flow (\$M) } & \multicolumn{10}{|c|}{ Year (N) } & \multirow[b]{2}{*}{10} \\
\hline & 0 & 1 & 2 & 3 & 4 & 5 & 6 & 7 & 8 & 9 & \\
\hline Total Revenue from sale of AJF & 0 & 439.30 & 439.30 & 439.30 & 439.30 & 439.30 & 439.30 & 439.30 & 439.30 & 439.30 & 439.30 \\
\hline Total Revenue from emissions benefit & 0 & 523.24 & 523.2 & 523.2 & 523.2 & 523.24 & 523.2 & 523.2 & 523.2 & 523.24 & 523.24 \\
\hline Total COP of AJF & 0 & 648.49 & 648.5 & 648.5 & 648.5 & 648.49 & 648.5 & 648.5 & 648.5 & 648.49 & 648.49 \\
\hline EBITDA & 0 & 314.05 & 314.05 & 314.05 & 314.05 & 314.05 & 314.05 & 314.05 & 314.05 & 314.05 & 314.05 \\
\hline Discount Factor & 1.00 & 1.07 & 1.14 & 1.23 & 1.31 & 1.40 & 1.50 & 1.61 & 1.72 & 1.84 & 1.97 \\
\hline Discounted EBITDA & 0.00 & 293.50 & 274.30 & 256.36 & 239.59 & 223.91 & 209.26 & 195.57 & 182.78 & 170.82 & 159.65 \\
\hline Terminal Value & & & & & & & & & & & 4486.43 \\
\hline Upfront Capital Cost & -947.30 & & & & & & & & & & \\
\hline NPV & 5744.88 & & & & & & & & & & \\
\hline
\end{tabular}

ASSUMPTIONS:

Algae oil content $=60 \%$

System productivity $=60 \mathrm{~g} / \mathrm{m} 2 /$ day

Total pond size $=4050$ ha

Total algal oil produced $=[(60 \mathrm{~g} / \mathrm{m} 2 /$ day $) \times(10,000 \mathrm{~m} 2 / \mathrm{ha}) \times(4050 \mathrm{ha}) \times(0.60 \mathrm{gAlgal}-$ oi $/ \mathrm{gAlgae}) \times(330$ days $/$ year $)] /[1000 \mathrm{~g} / \mathrm{kg}]=481,140,000 \mathrm{Kg} / \mathrm{Y}$

Algal oil denisty $=920 \mathrm{~kg} / \mathrm{m} 3$

Total oil produced $=[(481,140,000 \mathrm{~kg}) /(920 \mathrm{~kg} / \mathrm{m3})] \times(1000 \mathrm{~L} / \mathrm{m} 3)=522,978,260.90 \mathrm{~L} / \mathrm{Y}$

COP of AJF $=(\$ 0.90 / \mathrm{L})+(\$ 0.34 / \mathrm{L})=\$ 1.24 / \mathrm{L}$

Total COP $=(522,978,260.90 \mathrm{~L} / \mathrm{Y}) \times(\$ 1.24 / \mathrm{L})=\$ 648,493,043.50$

Total Revenue from Sale of AJF $=489,460,834.20 \mathrm{~L} / \mathrm{Y}) \times(\$ 0.84 / \mathrm{L})=\$ 439,301,739.10$

Average price of Jetfuel for $2013=\$ 132.80 / \mathrm{bbl}$ or $\$ 0.84 / \mathrm{L}$

Emissions price $=\$ 400 / \mathrm{tCO} 2 \mathrm{e}$

Energy Density of Bio-SPK $=43.5 \mathrm{MJ} / \mathrm{kg}$

Total production per year by energy $(\mathrm{MJ})=(481,140,000 \mathrm{Kg} / \mathrm{Y}) \times(43.5 \mathrm{MJ} / \mathrm{kg})=2,092,9590,000 \mathrm{MJ}$

Life-Cylce Emission intensity of AJF $=25 \mathrm{gCO} e / \mathrm{MJ}$

Total Life-Cycle Emission per year from AJF $=(2,092,9590,000 \mathrm{MJ}) \times(25 \mathrm{gCO} 2 \mathrm{e} / \mathrm{MJ})=523,239,750,000 \mathrm{gCO} e$

Life-Cycle Emissions intensity of PJF $=87.5 \mathrm{gCO} e / \mathrm{MJ}$

Total Life-Cycle Emission per year from PJF $=(2,092,9590,000 \mathrm{MJ}) \times(87.5 \mathrm{gCO} \mathrm{e} / \mathrm{MJ})=1,831,339,125,000 \mathrm{gCO} \mathrm{e}$

Total Life-Cycle Emission benefit per year $=(1,831,339,125,000)-(523,239,750,000)=1,308,099,375,000$ or 1,308,099.38 tCO2

Total Monetized Life-Cycle Emission Benefit per year $=(1,308,099.38$ tCO2 $) \times(\$ 400 / \mathrm{tCO} 2)=\$ 523,239,7$

WACC $=7 \%$

Total UCC $=\$ 947.3 \mathrm{M}$ 


\section{Calculations for Algal Oil Content, Productivity \& Carbon Tax - @ 60\%, $60 \mathrm{~g} / \mathrm{m}^{2} /$ day \& $\$ 800 / \mathrm{tCO}_{2} \mathrm{e}$}

HIGH GROWTH

$\quad$ Source of Cash Flow (\$M)
Total Revenue from sale of AJF
Total Revenue from emissions benefit
Total COP of AJF
EBITDA
Discount Factor
Discounted EBITDA
Terminal Value
Upfront Capital Cost
NPV

ALGAL OIL CONTENT 60\% \& PRODUCTIVITY $60 \mathrm{~g} / \mathrm{m2} /$ day \& Carbon Tax @ $\$ 800 / \mathrm{tCO} e$

\begin{tabular}{ccccccccccc}
\multicolumn{10}{c}{ Year (N) } \\
0 & 1 & 2 & 3 & 4 & 5 & 6 & 7 & 8 & 9 & 10 \\
0 & 439.30 & 439.30 & 439.30 & 439.30 & 439.30 & 439.30 & 439.30 & 439.30 & 439.30 & 439.30 \\
0 & $1,046.48$ & $1,046.48$ & $10,446.48$ & $\# \# \# \# \# \#$ & $1,046.48$ & $1,046.48$ & $1,046.48$ & $\# \# \# \# \# \#$ & $1,046.48$ & $1,046.48$ \\
0 & 648.49 & 648.5 & 648.49 & 648.5 & 648.49 & 648.5 & 648.49 & 648.5 & 648.49 & 648.49 \\
0 & 837.29 & 837.29 & 10237.29 & 837.29 & 837.29 & 837.29 & 837.29 & 837.29 & 837.29 & 837.29 \\
1.00 & 1.07 & 1.14 & 1.23 & 1.31 & 1.40 & 1.50 & 1.61 & 1.72 & 1.84 & 1.97 \\
0.00 & 782.51 & 731.32 & 8356.68 & 638.76 & 596.98 & 557.92 & 521.42 & 487.31 & 455.43 & 425.64 \\
& & & & & & & & & & 11961.29 \\
-947.30 & & & & & & & & & &
\end{tabular}

\section{ASSUMPTIONS:}

Algae oil content $=60 \%$

System productivity $=60 \mathrm{~g} / \mathrm{m} 2 /$ day

Total pond size $=4050$ ha

Total algal oil produced $=[(60 \mathrm{~g} / \mathrm{m} 2 /$ day $) \times(10,000 \mathrm{~m} 2 / \mathrm{ha}) \times(4050 \mathrm{ha}) \times(0.60 \mathrm{gAlgal}-$ oi $/ \mathrm{gAlgae}) \times(330$ days $/$ vear $)] /[1000 \mathrm{~g} / \mathrm{kg}]=481,140,000 \mathrm{Kg} / \mathrm{Y}$ Algal oil denisty $=920 \mathrm{~kg} / \mathrm{m3}$

Total oil produced $=[(481,140,000 \mathrm{~kg}) /(920 \mathrm{~kg} / \mathrm{m3})] \times(1000 \mathrm{~L} / \mathrm{m} 3)=522,978,260.90 \mathrm{~L} / \mathrm{Y}$

COP of AJF $=(\$ 0.90 / \mathrm{L})+(\$ 0.34 / \mathrm{L})=\$ 1.24 / \mathrm{L}$

Total COP $=(522,978,260.90 \mathrm{~L} / \mathrm{Y}) \times(\$ 1.24 / \mathrm{L})=\$ 648,493,043.50$

Total Revenue from Sale of AJF $=489,460,834.20 \mathrm{~L} / \mathrm{Y}) \times(\$ 0.84 / \mathrm{L})=\$ 439,301,739.10$

Average price of Jetfuel for $2013=\$ 132.80 / \mathrm{bbl}$ or $\$ 0.84 / \mathrm{L}$

Emissions price $=\$ 800 / \mathrm{tCO} 2 \mathrm{e}$

Energy Density of Bio-SPK $=43.5 \mathrm{MJ} / \mathrm{kg}$

Total production per year by energy $(\mathrm{MJ})=(481,140,000 \mathrm{Kg} / \mathrm{Y}) \times(43.5 \mathrm{MJ} / \mathrm{kg})=2,092,9590,000 \mathrm{MJ}$

Life-Cylce Emission intensity of AJF $=25 \mathrm{gCO} e / \mathrm{MJ}$

Total Life-Cycle Emission per year from AJF $=(2,092,9590,000 \mathrm{MJ}) \times(25 \mathrm{gCO} e / \mathrm{MJ})=523,239,750,000 \mathrm{gCO} e$

Life-Cycle Emissions intensity of PJF $=87.5 \mathrm{gCO} 2 \mathrm{e} / \mathrm{MJ}$

Total Life-Cycle Emission per year from PJF $=(2,092,9590,000 \mathrm{MJ}) \times(87.5 \mathrm{gCO} 2 \mathrm{e} / \mathrm{MJ})=1,831,339,125,000 \mathrm{gCO} \mathrm{e}$

Total Life-Cycle Emission benefit per year $=(1,831,339,125,000)-(523,239,750,000)=1,308,099,375,000$ or 1,308,099.38 tCO2

Total Monetized Life-Cycle Emission Benefit per year $=(1,308,099.38 \mathrm{tCO}) \times(\$ 800 / \mathrm{tCO} 2)=\$ 1,046,479,504$

WACC $=7 \%$

Total UCC $=\$ 947.3 \mathrm{M}$ 


\section{BIBLIOGRAPHY}

1. (2008). Climate action plan. British Columbia Ministry on Environment.

2. (2011). Beginners guide to aviation biofuels. Air Transport Action Group, Geneva, Switzerland.

3. (2010). Government's unique role in sustainable aviation fuel. Sustainable Aviation Fuel Users Group.

4. (2009). Powering future of flights. Air Transport Action Group, Geneva, Switzerland

5. (2011). Technology road map: biofuel for transport. International Energy Association. Paris, France.

6. (2012). Aviation benefits beyond borders. Air Transport Action Group, Geneva, Switzerland

7. (2012). Making progress on B.C.'s climate action plan. British Columbia Ministry on Environment.

8. (2012). Trouble in the air, trouble on the ground. www.economist.com . Retrieved August 2013.

9. (February 2012). Special fuel report. International Air Transport Association. www.iata.org . Retrieved Sept 2012.

10. (Jan 2009). Demonstrating the power of algae to fuel aviation (Media Release). Air Transport Action Group, Geneva, Switzerland.

11. Awudu, I., \& Zhang, J. (2012). Uncertainties and sustainability concepts in biofuel supply chain management: A review. Renewable and Sustainable Energy Reviews, 16(2), 1359-1368.

12. Azar, C., \& Sandén, B. A. (2011). The elusive quest for technology-neutral policies. Environmental Innovation and Societal Transitions, 1, 135-139.

13. Bürer, M. J., \& Wüstenhagen, R. (2009). Which renewable energy policy is a venture capitalist's best friend? Empirical evidence from a survey of international cleantech investors. Energy Policy, 37(12), 4997-5006.

14. Carriquiry, M. A., Du, X., \& Timilsina, G. R. (2011). Second generation biofuels: economics and policies. Energy Policy, 39(7), 4222-4234.

15. Dallara, E. S., Kroo, J. M., \& Waitz, I. A. (2011). Metric for Comparing Lifetime average Climate Impact of Aircraft. AIAA Journal, 49(8), 1600-1613.

16. Davis, R., Aden, A., \& Pienkos, P. T. (2011). Techno-economic analysis of autotrophic microalgae for fuel production. Applied Energy, 88(10), 3524-3531.

17. (December 2012). Financial forecast report. IATA.

18. (December 2012). Report on alternative fuels. IATA.

19. (July 2013). International Energy Outlook. Energy Information Administration. http://www.eia.gov/forecasts/ieo/transportation.cfm . Retrieved December 2013.

20. Elgowainy, A., Han, J., Wang, M., Carter, N., Stratton, R., Hileman, J., Malwitz, A., \& Balasubramanian, S. June 2012. Life-cycle analysis f alternative aviation fuels in GREET. Argonne National Laboratory.

21. Fabozzi, F. J., Drake, P. P. (2009). Finance: Capital markets, financial management, and investment management. John Wiley \& Sons.

22. Fairley, P. (2011). Introduction: Next generation biofuels. Nature, 474(7352), S2-S5.

23. Fishman, D., Frank, E. D., Wigmosta, M. S., Aden, A., Coleman, A. M., Pienkos, P. T., \& Wang, M. Q. (2012). Renewable diesel from algal lipids: an integrated baseline for cost, emissions, and resource potential from a harmonized model. Argonne National Laboratory.

24. Flight path to sustainable aviation. 2011. CSIRO.

25. Frank, E., Wang, M., Han, J., Elgowainy, A., \& Ralou-Rivera, I. (2011). Introduction to Algal Fuel LCA in GREET. Argonne National Laboratory.

26. Gallagher, B. J. (2011). The economics of producing biodiesel from algae. Renewable Energy, 36(1), 158-162.

27. Graham-Rowe, D. (2011). Agriculture: Beyond food versus fuel. Nature,474(7352), S6-S8.

28. Graham, P., Reedman, L., Roriguez, L., Raison, J., Braid, A., Haritos, V., Brinsmead, T., Hayward, J., Taylor, J., \& O'Connell, D. 2011. CSIRO sustainable aviation fuels road map: data assumptions and modeling. CSIRO.

29. Greenwell, H. C., Laurens, L. M. L., Shields, R. J., Lovitt, R. W., \& Flynn, K. J. (2010). Placing microalgae on the biofuels priority list: a review of the technological challenges. Journal of the Royal Society Interface, 7(46), 703-726.

30. Grierson, S., \& Strezov, V. (2012). Life-cycle assessment of the microalgae biofuel value chain: a critical review of existing studies. BIONATURE 2012, The Third International Conference on Bioenvironment, Biodiversity and Renewable Energies, 1-6.

31. Henriques, I., \& Sadorsky, P. (2008). Oil prices and the stock prices of alternative energy companies. Energy Economics, 30(3), 998-1010. 
32. Hilmena, J. I., Ortiz, D. S., Bartis, J. T., Wong, H. S., Donhoo, P. E., Weiss, M. A., \& Walktz, I. A. (2009). Near-term feasibility of alternative jet fuels. PARTNER.

33. How the carbon tax works. www.fin.gov.ca . Retrieved September 2012.

34. Kinder, J. D., \& Rahmes, T. (2009). Evaluation of bio-derived synthetic paraffinic kerosene (BioSPK). Sustainable Biofuels Research \& Technology Program, The Boeing Company.

35. Kivits, R., Charles, M. B., \& Ryan, N. (2010). A post-carbon aviation future: Airports and the transition to a cleaner aviation sector. Futures, 42(3), 199-211.

36. Knight, E., \& Nicholas, H. (2011). Clean energy technology and the role of non-carbon price based policy: an evolutionary perspective. Center for Climate Economics \& Policy, The Australian National University.

37. Knoshaug, E. P., \& Darzins, A. (2011). Algal biofuels: the process. Chemical Engineering Progress, 107(3), 37-47.

38. Krammer, P., Dray, L., \& Köhler, M. O. (2013). Climate-neutrality versus carbon-neutrality for aviation biofuel policy. Transportation Research Part D: Transport and Environment, 23, 64-72.

39. Lin, B., \& Li, X. (2011). The effect of carbon tax on per capita $\mathrm{CO}_{2}$ emissions. Energy Policy, 39(9), 5137-5146.

40. Masini, A., \& Menichetti, E. (2013). Investment decisions in the renewable energy sector: An analysis of non-financial drivers. Technological Forecasting and Social Change, 80, 510-524.

41. Newell, R. G. (2010). The role of markets and policies in delivering innovation for climate change mitigation. Oxford Review of Economic Policy, 26(2), 253-269.

42. Ng, J. H., Ng, H. K., \& Gan, S. (2010). Recent trends in policies, socioeconomy and future directions of the biodiesel industry. Clean Technologies and Environmental Policy, 12(3), 213-238.

43. Nygren, E., Aleklett, K., \& Höök, M. (2009). Aviation fuel and future oil production scenarios. Energy Policy, 37(10), 4003-4010.

44. O'Connell, D., \& Haritos, V. S. (2010). Conceptual investment framework for biofuels and biorefineries research and development. Biofuels, 1(1), 201-216.

45. Packer, M. (2009). Algal capture of carbon dioxide; biomass generation as a tool for greenhouse gas mitigation with reference to New Zealand energy strategy and policy. Energy Policy, 37(9), 34283437.

46. Pearlson, M., Wollersheim, C., \& Hileman, J. (2013). A techno-economic review of hydroprocessed renewable esters and fatty acids for jet fuel production. Biofuels, Bioproducts and Biorefining, 7(1), 89-96.

47. Preiss, M. R., \& Kowalski, S. P. (2010). Algae and Biodiesel: Patenting energized as green goes commercial. Journal of Commercial Biotechnology,16(4), 293-312.

48. Richardson, J. W., Johnson, M. D., \& Outlaw, J. L. (2012). Economic comparison of open pond raceways to photo bio-reactors for profitable production of algae for transportation fuels in the Southwest. Algal Research,1(1), 93-100.

49. Rosenbaum, J., \& Pearl, J. (2009). Investment banking. New Jersey. Wiley \& Sons.

50. Sadorsky, P. (2009). Renewable energy consumption, $\mathrm{CO}_{2}$ emissions and oil prices in the G7 countries. Energy Economics, 31(3), 456-462.

51. Schenk, P. M., Thomas-Hall, S. R., Stephens, E., Marx, U. C., Mussgnug, J. H., Posten, C., ... \& Hankamer, B. (2008). Second generation biofuels: high-efficiency microalgae for biodiesel production. Bioenergy Research, 1(1), 20-43.

52. Sgouridis, S., Bonnefoy, P. A., \& Hansman, R. J. (2011). Air transportation in a carbon constrained world: Long-term dynamics of policies and strategies for mitigating the carbon footprint of commercial aviation. Transportation Research Part A: Policy and Practice, 45(10), 1077-1091.

53. Shiho, M., Kawachi, M., Horioka, K., Nishita, Y., Ohashi, K., Kaya, K., \& Watanabe, M. M. (2012). Business evaluation of a green microalgae Botryococcus Braunii oil production system. Procedia Environmental Sciences, 15, 90-109.

54. Singh, J., \& Gu, S. (2010). Commercialization potential of microalgae for biofuels production. Renewable and Sustainable Energy Reviews, 14(9), 2596-2610.

55. Sodero, S. (2011). Policy in motion: reassembling carbon pricing policy development in the personal transport sector in British Columbia. Journal of Transport Geography, 19(6), 1474-1481.

56. Sorda, G., Banse, M., \& Kemfert, C. (2010). An overview of biofuel policies across the world. Energy Policy, 38(11), 6977-6988.

57. Stephens, E., Ross, I. L., King, Z., Mussgnug, J. H., Kruse, O., Posten, C., \& Hankamer, B. (2010). An economic and technical evaluation of microalgal biofuels. Nature Biotechnology, 28(2), 126-128.

58. Sun, A., Davis, R., Starbuck, M., Ben-Amotz, A., Pate, R., \& Pienkos, P. T. (2011). Comparative cost analysis of algal oil production for biofuels. Energy, 36(8), 5169-5179. 
59. Taylor, B., Xiao, N., Sikorski, J., Yong, M., Harris, T., Helme, T., ... \& Kraft, M. (2013). Techno-economic assessment of carbon-negative algal biodiesel for transport solutions. Applied Energy, 106, 262-274.

60. Timilsina, G. R., Csordás, S., \& Mevel, S. (2011). When does a carbon tax on fossil fuels stimulate biofuels?. Ecological Economics, 70(12), 2400-2415.

61. Vertes, A., Qureshi, N., Blachek, H. P., \& Tukawa, H. (2010). West Sussex. John Wiley \& Sons.

62. Walsh, P.R. (2012). Innovation nirvana or innovation wasteland? Identifying commercialization strategies for small and medium renewable energy enterprises. Technovation, 32(1), 32-42.

63. Winchester, N., McConnachie, D., Wollersheim., \& Waltz, I. A. (2013). Market cost of renewable jet fuel adoption in the United States. PARTNER. 